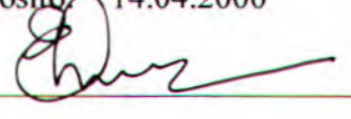

\title{
Sobre pares de folheações em variedades de dimensão 2
}

\author{
Regilene Delazari dos Santos Oliveira
}

\author{
Orientador: Prof. Dr. Farid Tari
}

Tese apresentada ao Instituto de Ciências Matemáticas e de Computação - ICMC-USP, como parte dos requisitos para obtenção do título de Doutor em Ciências - Área: Matemática.

USP - São Carlos

Abril de 2000 
Aos meus pais, Antonio e Glauci, à minha irmã, Lucilene e ao meu marido, Milton, pelo apoio e carinho. 
"Algumas vezes acreditamos que terminamos mais uma etapa em nossa vida enquanto que na verdade, estamos apenas recomeçando uma nova." Que Deus me ilumine nessa nova etapa que estou iniciando hoje! 


\section{AGRADECIMENTOS}

$\grave{A}$ Deus, por ter estado comigo em todos os dias de minha vida.

Aos meus pais e minha irmã, por acreditarem em mim, pelo apoio, incentivo e carinho, sem os quais eu não seria quem sou hoje.

À Tinho agradeço de modo especial, por ter estado sempre presente, dando-me força e carinho e pela enorme paciência em momentos em que fui realmente uma pessoa insuportável.

Ao Farid, meu orientador, primeiramente por ter proposto este trabalho em minha tese e depois por acreditar em minha capacidade, por apoiar-me nas dificuldades e pela amizade. Agradeço também pela hospitalidade com que ele e sua esposa, Stephanie, me receberam quando visitei a Universidade de Liverpool.

À todos os professores e funcionários do Departamento de Matemática do ICMC - USP, por terem me acolhido, pela amizade cultivada e pela atenção dispensadan durante todo o desenvolvimento deste trabalho. Agradeço também ao Departamento de Matemática da Universidade de Liverpool pela hospitalidade com que me receberam durante a fase final deste trabalho de pesquisa.

Agradeço de modo especial aos professores Angela Maria Sitta, Maria Aparecida Soares Ruas, James Willian Bruce, Solange Mancini, e Miriam Manoel por valiosas sugestões e conversas.

Agradeço também a todos os professores e amigos do Departamento de Matemática da Universidade Estadual Paulista, Campus de Rio Claro, que muito contribuiram na minha formação, em especial 'a Profa. Rosa Lúcia Sverzut Baroni, por ter sempre me incentivado.

À todos os amigos que fiz durante minha vida universitária, em especial à Maria do Carmo, Suzeli, Luciana e Fernando, Ana Claudia, Jean e Felipe, Vania e Chico, Marcão, Maria Alice, Vera, José, Eliane e Marcio, Leunilce e Daniela

À Fundação de Amparo à Pesquisa do Estado de São Paulo (FAPESP) pelo suporte financeiro. 


\begin{abstract}
In this thesis we study in a systematic way pairs of germs of foliations defined by differential 1-forms in the plane using three different techniques: singularity theory, polar blowing up and formal reduction.

Using singularity theory, we present a smooth and complete classification of pairs of germs of finite codimension of regular and regular/singular exact foliations in the plane and in 2dimensional manifolds with regular boundary. We investigate the geometry of the classified pairs and associate invariants to them.

With the blowing up method we obtain topological models of pairs of singular and regular/singular foliations in the plane, when the singularity is of type saddle, node or focus. We also study theses cases using the formal reduction technique.

Finally, we prove a desingularization theorem for pairs of germs of differential 1-forms in the plane, similar to Seidenberg and Dumortier's theorems for 1-form.
\end{abstract}




\section{Resumo}

Nesta tese estudamos de maneira sistemática pares de germes de folheações determinadas por 1-formas diferenciais no plano, usando três diferentes técnicas: a teoria de singularidades, o método do blowing up polar e a redução formal.

Usando a teoria de singularidades, apresentamos uma classificação suave e completa dos pares de germes de codimensão finita de folheações regulares ou da forma regular/singular-exata no plano e em variedades bidimensionais com bordo regular. Fizemos um estudo geométrico dos pares encontrados nas classificações e associamos invariantes à tais pares.

Com o método do blowing up obtivemos um estudo topológico dos pares de folheações regular/singular e singular/singular com singularidades do tipo sela, nó ou foco. Usamos também o método da redução formal para estudar estes casos.

Finalmente, estabelecemos um teorema de desingularização de pares de 1-formas diferenciais no plano, análogo aos teoremas de Seidenberg e Dumortier sobre a desingularização de 1-formas diferenciais no plano. 


\section{Sumário}

Introdução

1 Classificação suave dos pares de folheações regulares em variedades de dimensão 2

1.1 Preliminares sobre a teoria de singularidades . . . . . . . . . . . . 4

1.1.1 Grupos de Mather . . . . . . . . . . . . . . . . 5

1.1 .2 Determinação finita . . . . . . . . . . . . . . . 7

1.1.3 Desdobramentos versais . . . . . . . . . . . . . 8

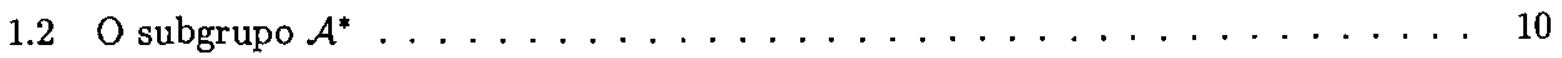

1.2 .1 O subgrupo geométrico $\mathcal{A}^{*}$ e suas propriedades $\ldots \ldots \ldots \ldots \ldots$

$1.3 \mathcal{A}^{*}$-classificação de germes regulares $\mathbb{R}^{2}, 0 \rightarrow \mathbb{R}, 0 \ldots \ldots \ldots \ldots$

1.3.1 Classificação de germes com contato 1 e 2 na origem . . . . . . . . . 19

1.3.2 Classificação de germes com contato 3 na origem . . . . . . . . . . 20

1.3.3 Contato $\geq 4$ na origem . . . . . . . . . . . . 23

1.4 Classificação dos pares de 1 -formas regulares $\ldots \ldots \ldots \ldots \ldots$. . . . . . . 27

1.5 Estudo geométrico . . . . . . . . . . . . . . . . 30

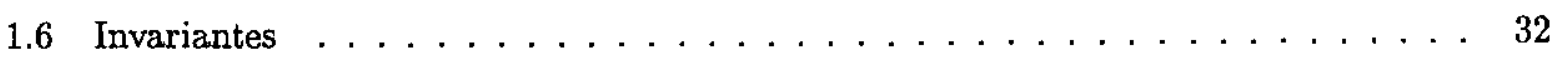

1.7 Eliminação do módulo na classificação suave . . . . . . . . . . . . . 35

2 Classificação suave de pares de 1-formas diferenciais $(d y, d f)$ em variedades de $\begin{array}{ll}\text { dimensão } 2 & 40\end{array}$

2.1 Classificação. . . . . . . . . . . . . . . . . . . . . 40

2.2 Estudo geométrico . . . . . . . . . . . . . . . . 45

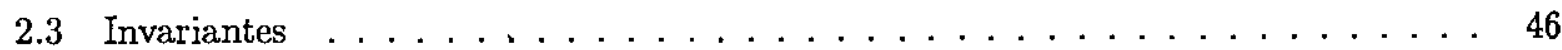

3 Classificação suave de pares de folheações $(d y, d f)$ sobre variedades de dimensão 2 com bordo regular 49

$3.1 B$-Classificação dos germes $\mathbb{R}^{2}, 0 \rightarrow \mathbb{R}, 0 \ldots \ldots \ldots \ldots \ldots$

3.1.1 Classificação dos pares de folheações . . . . . . . . . . . . . 60 
3.1 .2 Estudo geométrico . . . . . . . . . . . . . . . 62

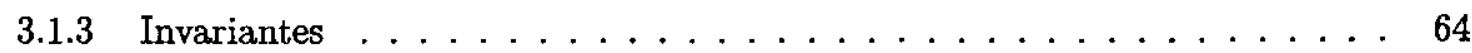

$3.2 \mathcal{B}_{\alpha}$-Classificação de germes $\mathbb{R}^{2}, 0 \rightarrow \mathbb{R}, 0 \ldots \ldots \ldots \ldots$

3.2 .1 Caso regular . . . . . . . . . . . . . . 67

3.2 .2 Estudo geométrico . . . . . . . . . . . . . . . 68

3.2 .3 Caso singular . . . . . . . . . . . . . . . 71

3.2 .4 Estudo geométrico . . . . . . . . . . . . . . . 71

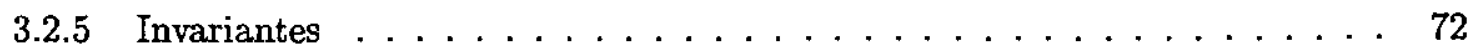

4 Classificação topológica de pares de folheaçōes em variedades de dimensão $2 \mathbf{7 4}$

4.1 Genericidade e transversalidade . . . . . . . . . . . . . . 75

4.2 Método de blowing up polar . . . . . . . . . . . . . . . . 77

4.3 Resultados auxiliares . . . . . . . . . . . . . . . . . . 78

4.4 Classificação topológica de pares de folheações no plano . . . . . . . . . . 81

5 Classificação formal de pares de folheações no plano 90

5.1 Estudo Formal . . . . . . . . . . . . . . . . . . . . 91

6 Desingularização de pares de 1-formas diferenciais no plano 95

6.1 Desingularização de uma 1-forma diferencial . . . . . . . . . . . . . 95

6.2 Desingularização de pares de 1-formas diferencias . . . . . . . . . . . . . 97

6.2 .1 o caso analítico $\ldots \ldots \ldots \ldots \ldots \ldots$

6.2 .2 O caso suave $\ldots \ldots \ldots \ldots \ldots$. . . . . . . . . . . . . . . . . .

$\begin{array}{ll}\text { Referências Bibliográficas } & 104\end{array}$ 


\section{Introdução}

O objetivo do nosso trabalho é estudar de maneira sistemática pares de folheações determinadas por 1-formas diferenciais no plano, ou equivalentemente, pares de campos de direções no plano.

Alguns modelos de pares de campos de direções foram apresentados em trabalhos anteriores. Em [37], Teixeira caracterizou pares estáveis $(\xi, f)$, onde $\xi$ é um campo de vetores e $f$ uma função real cujas singularidades são disjuntas das singularidades de $\xi$ e provou a existência de um par estruturalmente estável $(\xi, f)$ na esfera. Davydov [16] provou que pares estruturalmente estáveis em [37] são de fato estáveis por difeomorfismos e exibiu formas normais para tais pares. Além disso Davydov mostra aplicaçōes do estudo de pares de 1-formas diferenciais no plano na Teoria de Controle.

Bruce e Fidal em [6] classificaram alguns pares de campos de direções para obter formas normais topológicas para equações diferenciais binárias (EDB), isto é equações da forma $a(x, y) d y^{2}+2 b(x, y) d x d y+c(x, y) d x^{2}=0$, onde $a, b, c$ são germes de funções suaves que se anulam em $(0,0)$. Em [6] o caso $c=-a$ é estudado. Esta condição é substituída em [11] pela hipótese da função discriminante possuir singularidade do Tipo Morse. Estas situações ocorrem quando estudamos, por exemplo, a configuração das linhas de curvatura na vizinhança de pontos umbílicos. Outros exemplos de EDB podem ser encontrados em [12].

O método utilizado em [6] e [11] é o seguinte. O campo de direções bivalorado no plano determinado pela equação é levantado a um campo univalorado no cilíndro $M$, sendo que o círculo central do cilíndro é o levantamento da origem. Além disso pontos sobre o cilíndro que correspondem ao mesmo ponto no plano são identificados, definindo assim uma involução em $M$. O problema se reduz a classificar certos pares de campos de direçōes no espaco quociente, a faixa de Mobius $M^{\prime}$. A linha central da faixa de Mobius é uma curva integral comum a ambos os campos, então modelos locais de pares de campos de direções regulares ou regular/singular com uma curva integral em comium são dados em [6].

Em [30] Michel estudou pares de campos singulares com uma singularidade do tipo sela ou nó e com as duas separatrizes em comum. Em [7] é dado um modelo para o caso onde os campos têm os mesmos autovalores e uma separatriz em comum.

Observamos que os casos onde as 1-formas têm uma folha em comum são infinitamente 
degenerados, no entanto acontecem naturalmente quando aplicamos um "blowing up"ao par de 1-formas.

Neste trabalho utilizamos três diferentes técnicas para estudar pares de folheaçōes, a teoria de singularidades, o método do blowing up polar e a redução formal.

A teoria de singularidades oferece técnicas poderosas para o estudo dos pares $(\alpha, \beta)$ regulares ou $\alpha$ regular e $\beta$ exata. Em ambos os casos podemos supor que $\alpha=d y$ e $\beta=d f$, onde $f$ é um germe de função de classe $C^{\infty}$. Então a classificação do par $(\alpha, \beta)$ se reduz a classificação de $f$ por mudanças de coordenadas que preservam as retas horizontais, isto é, as folhas de $d y$.

Este formalismo nos possibilita ainda obter modelos para as deformações de $(\alpha, \beta)$ e meios para associar invariantes a tais pares.

No capítulo 1 , estudamos o caso onde $\alpha$ e $\beta$ são regulares e no capítulo 2 , o caso $\beta$ singular exata. Obtemos uma classificação suave e completa dos germes de codimenssão finita e estudamos a geometria dos pares classificados.

Estendemos estes resultados no capítulo 3 para os pares acima sobre variedades com bordo regular. Tratamos dois casos, primeiro quando o bordo não é uma folha de $\alpha$ nem de $\beta$ e depois, quando o bordo coincide com a folha da 1-forma $\alpha$ na origem.

Usando o método do blowing up polar, obtemos no capítulo 4, modelos topológicos para os pares de 1-formas regular/singular e singular/singular com singularidade do tipo sela, nó ou foco, no plano. A idéia usada aqui foi estender a técnica do blowing up de um germe de 1-forma para dois germes, assim substituímos uma singularidade comum às 1-formas por um círculo, chamado de curva excepcional, que é uma folha comum ao par de 1-formas. Obtendo modelos locais para os pontos ao longo da curva excepcional, colamo-os através das técnicas desenvolvidas por Bruce e Fidal [6], e por blowing down temos modelos topológicos.

Exploramos também a técnica da redução formal no capítulo 5. Mostramos que nos casos regular/singular ou singular/singular com singularidade do tipo sela, nó ou foco, podemos reduzir os pares à formas mais simples. A redução formal consiste em eliminar sucessivamente os termos de ordem $k(k \geq 2)$ nas funções que definem as 1-formas diferenciais. A questão da convergência deste processo ainda esta aberta para os pares acima e no caso geral.

Estudamos no capitulo 6, um teorema de desingularização dos pares de 1-formas diferenciais no plano, análogo aos teoremas de Seidenberg e Dumortier sobre a redução das singularidades de uma 1-forma diferencial no plano.

Algumas das figuras deste trabalho foram obtida usando "The Liverpool Surface Modeling Package" [32]. 


\section{Capítulo 1}

\section{Classificação suave dos pares de folheações regulares em variedades}

\section{de dimensão 2}

Neste capítulo apresentamos uma classificação suave e completa de pares de germes de 1-formas diferenciais regulares $(\alpha, \beta)$ de codimensão finita, definidos na vizinhança de um ponto $p$ de uma variedade $M$ de dimensão 2. A classificação é obtida através das técnicas da teoria de singularidades, que nos possibilita apresentar as formas normais, o grau de determinação, as perturbaçōes versais, determinar a codimensão, estudar a geometria dos pares classificados e associarmos invariantes aos pares de 1-formas diferenciais no plano.

Nesta classificação nos restringimos ao caso onde a folha de $\beta$ na origem tem contato finito com a folha de $\alpha$. O estudo dos pares com contato infinito (uma folha em comum) pode ser encontrado em $[6,30,37,38]$, por exemplo.

Antes de dar início ao nosso estudo sobre pares de 1-formas vamos ver definições básicas sobre 1-formas diferenciais no plano.

Por uma 1-forma diferencial em um conjunto aberto do $\mathbb{R}^{2}$ entendemos uma aplicação $\alpha$, que associa a cada ponto do aberto um funcional linear que denotamos por $\alpha(x, y)=a_{1}(x, y) d x+$ $a_{2}(x, y) d y$, onde $a_{1}, a_{2}$ são funções $C^{\infty}$ neste aberto. Note que dada uma 1-forma diferencial no plano temos uma folheação do aberto cujas folhas são as soluções da equação diferencial $a_{1}(x, y) d x+a_{2}(x, y) d y=0$.

Enquanto que dado um aberto do $\mathbb{R}^{2}$, um campo de direções de classe $C^{\infty}$ é uma função $X$ de classe $C^{\infty}$ que associa a cada ponto $p$ um vetor $X(p) \in T_{p} M$, onde $\mathrm{X}$ pode ser identificado, numa vizinhança do aberto com a aplicação dada por $X(x, y)=A(x, y) \frac{\partial}{\partial x}+B(x, y) \frac{\partial}{\partial y}$, onde $A$, $B$, são os funçōes de classe $C^{\infty}$ que definem o campo.

. Uma curva $C$ é dita curva integral de um campo de direções se em cada ponto dela o vetor 
tangente coincide com o vetor associado pelo campo a este ponto. Dada uma 1-forma diferencial no plano podemos associar um campo de direções $\omega$, cujas curvas integrais são as folhas da 1-forma $\alpha$, denotado por

$$
\omega(x, y)=a_{2}(x, y) \frac{\partial}{\partial y}-a_{1}(x, y) \frac{\partial}{\partial x} .
$$

Neste sentido, o estudo dos campos de direções no plano é equivalente ao estudo das 1-formas diferenciais no plano.

Diremos ainda que uma 1-forma diferencial $\alpha$ definida em $U$ é singular em $p \in U$, se $\alpha(p)=0$, neste caso $p$ é dito ponto crítico, ou ponto singular ou ainda, uma singularidade de $\alpha$. Para os pontos $p$ onde $\alpha(p) \neq 0$ dizemos que $\alpha$ é regular, assim como o ponto $p$.

Definimos o $k$-jato da 1-forma $\alpha=A(x, y) d y+B(x, y) d x$ como $j^{k}(\alpha)=j^{k} A(x, y) d y+$ $j^{k} B(x, y) d x$, onde $j^{k} A$ e $j^{k} B$ representam o truncamento da expansão da série de Taylor de $A$ e $B$ respectivamente, ou seja, ignoramos os termos de grau maior do que $k$.

Podemos definir relações de equivalência no conjunto das 1-formas diferenciais. Dadas duas 1formas diferenciais $\alpha, \beta$ num aberto $U \subset \mathbb{R}^{2}$, diremos que $\alpha$ e $\beta$ são topologicamente equivalentes (respec. suavemente) se existe um homeomorfismo (respec. difeomorfismo) $h: U \rightarrow U$ levando as folhas de $\alpha$ às folhas de $\beta$. Aos representantes convenientes das classes de equivalência pela relação acima, chamamos modelos ou formas normais (note que podemos ainda multiplicar uma 1-forma $\alpha$ por uma função nāo nula e obter modelos equivalentes).

Estudamos agora preliminares sobre a teoria de singularidades, método utilizado na classificação dos pares de 1-formas neste capítulo.

\subsection{Preliminares sobre a teoria de singularidades}

Seja $x \in \mathbb{R}^{\mathrm{n}}$ e $f, g: U \subset \mathbb{R}^{\mathrm{n}} \rightarrow \mathbb{R}^{\mathrm{P}}$ aplicaçōes diferenciáveis definidas em uma vizinhança aberta $U$ de $x$. Dizemos que $f$ e $g$ são equivalentes e escrevemos $f \sim g$, se existir uma vizinhança $V$ de $x$ em $\mathbb{R}^{\mathrm{n}}$ tal que $f|V=g| V$, ou seja, se $f$ e $g$ coincidem em uma vizinhança $V$ de $x$. As classes de equivalência desta relação são chamadas germes de aplicações diferenciáveis de $\mathbb{R}^{\mathfrak{n}} \rightarrow \mathbb{R}^{p}$ em $x$ e os elementos de uma classe são chamados representantes do germe. Representantes do mesmo germe assumem o mesmo valor em $x$, assim como todas as suas derivadas: $D^{n} f(x)=$ $D^{n} g(x), \forall n \in \mathbb{N}$. Usa-se a notação $f: \mathbb{R}^{\mathbf{n}}, x \rightarrow \mathbb{R}^{\mathrm{p}}$ para indicar um germe de uma aplicação em $x$.

Consideramos, sem perda de generalidade, os germes em 0 de aplicações $\mathbb{R}^{n} \rightarrow \mathbb{R}$.

Observe que usando as operaçōes na meta $\mathbb{R}$, podemos somar e multiplicar tais aplicaçōes, então o conjunto dos germes adquire uma estrutura de anel, o qual denotamos por $\mathcal{E}_{n}$. Este anel tem um ideal $\mathcal{M}_{n}$ constituído dos germes de funções $\phi: \mathbb{R}^{\mathbf{n}}, 0 \rightarrow \mathbb{R}, 0$, ou seja, funções $\phi$ com $\phi(0)=0$. Este é o único ideal maximal do anel, logo $\mathcal{E}_{n}$ é um anel local. 
Sendo assim, dado um germe de aplicação $f: \mathbb{R}^{\mathrm{n}}, 0 \rightarrow \mathbb{R}^{\mathrm{p}}$ temos $f=\left(f_{1}, f_{2}, \ldots, f_{p}\right)$, com $f_{1}, \ldots, f_{p} \in \mathcal{E}_{n}$. Podemos identificar o espaço dos germes $\mathbb{R}^{\mathrm{n}}, 0 \rightarrow \mathbb{R}^{\mathrm{p}}$ com o $\mathcal{E}_{n}$-módulo livre $\mathcal{E}(n, p)=\mathcal{E}_{n} \times \ldots \times \mathcal{E}_{n}$ (p-cópias).

Podemos introduzir uma outra relação de equivalência entre as aplicações $\mathbb{R}^{\mathbf{n}}, 0 \rightarrow \mathbb{R}^{\mathbf{p}}$. Dado $x \in \mathbb{R}^{\mathrm{n}}$ e $f, g: \mathbb{R}^{\mathrm{n}}, \mathrm{x} \rightarrow \mathbb{R}^{\mathrm{p}}$ germes de aplicaçōes em $x$, dizemos que $f$ e $g$ têm o mesmo $k$-jato em $x$ se $f(x)=g(x)$ e para algum sistema local de coordenadas em $x \in \mathbb{R}^{\mathrm{n}}$ e $f(x) \in \mathbb{R}^{\mathrm{p}}$ todas as derivadas parciais de ordem $\leq k$ de $f$ e $g$ coincidem em $x$. Sendo assim esta definição depende apenas de $f$ e $g$. O conjunto de todos os $k$-jatos dos germes de aplicações $\mathbb{R}^{\mathrm{n}}, 0 \rightarrow \mathbb{R}^{\mathrm{p}}, 0$ (conjunto das aplicações polinomiais de grau $\leq k$ com termos constantes nulos) é denotado por $J^{k}(n, p)$. O conjunto dos germes de aplicaçōes $\mathbb{R}^{\mathrm{n}}, 0 \rightarrow \mathbb{R}, 0$ cujas derivadas de ordem $\leq k-1$ se anulam em 0 é denotado por $\mathcal{M}_{n}^{k}$.

Logo dada a definição acima temos

$$
J^{k}(n, p)=\frac{\mathcal{M}_{n} \mathcal{E}(n, p)}{\mathcal{M}_{n}^{k+1} \mathcal{E}(n, p)} .
$$

\subsubsection{Grupos de Mather}

Denotamos por $\mathcal{R}$ o grupo dos germes de difeomorfismos $\mathbb{R}^{\mathrm{n}}, 0 \rightarrow \mathbb{R}^{\mathrm{n}}, 0, \mathcal{L}$ o grupo dos germes de difeomorfismos $\mathbb{R}^{\mathrm{p}}, 0 \rightarrow \mathbb{R}^{\mathrm{p}}, 0$, e $\mathcal{A}$ o produto semi-direto $\mathcal{R} \times \mathcal{L}$. Estes grupos agem sobre $\mathcal{M}_{n} \cdot \mathcal{O}(n, p)$ por composição, como segue:

$$
\begin{aligned}
h . f & =f \circ h^{-1}, h \in \mathcal{R} \\
k . f & =k \circ f, k \in \mathcal{L} \\
(h, k) . f & =k \circ f \circ h^{-1}, \quad(h, k) \in \mathcal{A}
\end{aligned}
$$

onde $f \in \mathcal{M}_{n} . \mathcal{E}(n, p)$. O grupo $\mathcal{R}$ (resp. $\mathcal{L}$ ) é chamado também o grupo de mudanças de coordenadas na fonte (resp. na meta). Dessa forma, dados dois germes de aplicações $f, g \in$ $\mathcal{M}_{n} \cdot \mathcal{E}(n, p)$ diremos que $f$ e $g$ são $\mathcal{A}$-equivalentes se existirem $(h, k) \in \mathcal{A}$ tal que $g=k \circ f \circ h^{-1}$.

Por $\mathcal{C}$ denotamos o grupo de difeomorfismos $\mathbb{R}^{\mathrm{n}} \times \mathbb{R}^{\mathrm{p}}, 0 \rightarrow \mathbb{R}^{\mathrm{n}} \times \mathbb{R}^{\mathrm{p}}, 0$ que são escritos na forma $H(x, y)=(x, \bar{H}(x, y)) \operatorname{com} \bar{H}(x, 0)=0$ para $x \in \mathbb{R}^{\mathrm{n}}$ próximo à origem. A ação de $\mathcal{C}$ sobre $m_{n} \cdot \mathcal{E}(n, p)$ é definida como

$$
H . f(x)=H(x, f(x)), H \in \mathcal{C}, f \in m_{n} \cdot \mathcal{E}(n, p) .
$$

$\mathcal{C}$ pode ser visto como o grupo de difeomorfismos $\mathbb{R}^{\mathrm{p}}, 0 \rightarrow \mathbb{R}^{\mathrm{p}}, 0$ parametrizados para $x \in \mathbb{R}^{\mathrm{n}}$.

Temos ainda o grupo $\mathcal{K}$, o grupo dos germes de difeomorfismos $\mathbb{R}^{\mathrm{n}} \times \mathbb{R}^{\mathrm{p}}, 0 \rightarrow \mathbb{R}^{\mathrm{n}} \times \mathbb{R}^{\mathrm{p}}, 0$ que são escritos na forma

$$
H(x, y)=(h(x), \bar{H}(x, y))
$$


onde $h \in \mathcal{R}, \bar{H}(x ; 0)=0$ para $x \in \mathbb{R}^{\mathrm{n}}$ próximo à origem. A ação de $\mathcal{K}$ sobre $\mathcal{M}_{n} . \mathcal{E}(n, p)$ é definida como

$$
H . f(x)=H\left(h^{-1}(x), f\left(h^{-1}(x)\right)\right), H \in \mathcal{K}, f \in m_{n} \cdot \mathcal{E}(n, p) .
$$

$O$ grupo $\mathcal{K}$ é chamado grupo de contato. $O$ grupo $\mathcal{C}$ é um subgrupo normal de $\mathcal{K}$ e os grupos $\mathcal{R}, \mathcal{L}, \mathcal{A}$ podem ser identificados com subgrupos de $\mathcal{K}$.

O grupo de contato tem ainda uma interpretação geométrica (para tal interpretação ver [31]).

$\mathcal{R}, \mathcal{L}, \mathcal{A}, \mathcal{C}, \mathcal{K}$ são conhecidos como Grupos de Mather. Daqui por diante, quando uma afirmação for válida para qualquer um dos grupos de Mather acima, diremos apenas o grupo $\mathcal{G}$.

Vamos descrever os espaços tangentes às órbitas dos grupos de Mather. Faremos isso descrevendo primeiramente os espaços tangentes às órbitas dos grupos $\mathcal{R}, \mathcal{L}$.

Seja $\mathcal{G}$ um dos grupos de Mather. Definimos álgebra de Lie do grupo $\mathcal{G}$ como segue.

Considere $\phi:(-\epsilon, \epsilon) \times \mathbb{R}^{\mathrm{n}+\mathrm{p}}, 0 \rightarrow \mathbb{R}^{\mathrm{n}+\mathrm{p}}, 0$ uma curva em $\mathcal{G}$ tal que $\phi_{0}$ é a identidade em $\mathcal{G}$. Derivando-a temos um campo de vetores

$$
\left.x \rightarrow \frac{\partial \phi}{\partial t}(t, x)\right|_{t=0}
$$

O conjunto de todos estes campos é denotado por $L \mathcal{G}$ e é chamado a álgebra de Lie do grupo $\mathcal{G}$.

Então a álgebra de Lie do grupo $\mathcal{R}$ é dada por $L \mathcal{R}=\mathcal{M}_{n} \cdot \mathcal{E}_{n}$, afinal se $\phi_{0}$ é a identidade temos $\phi(t, x)=\left(x_{1}+t \alpha_{1}(t, x), \ldots, x_{n}+t \alpha_{n}(t, x)\right)$, entāo $\frac{\partial \phi}{\partial t}=\left(\alpha_{1}, \ldots, \alpha_{n}\right)$. Desde que $\phi_{t} \in \mathcal{R}$ e $\phi_{0}=I d$ temos que $\alpha_{i}(0,0)=0, \operatorname{logo} \alpha=\left(\alpha_{1}, \ldots, \alpha_{n}\right) \in \mathcal{M}_{n} \mathcal{E}_{n}$.

Analogamente temos que a álgebra de Lie do grupo $\mathcal{L}$ é dada por $L \mathcal{L}=\mathcal{M}_{p} \mathcal{E}_{p}$.

Para definir o espaço tangente às órbitas do grupo $\mathcal{G}$, podemos generalizar a noção geométrica de espaço tangente em um ponto $p$ para variedade de dimensão finita. Ou seja, dada uma variedade, tomamos uma curva suave passando por $\mathrm{p}$ em $t=0$, em seguida derivamos esta curva e avaliamos-a em 0 . O conjunto de todos os vetores obtidos dessa maneira é o espaço tangente a variedade ern $\mathrm{p}$.

Em nosso caso, consideramos um caminho na órbita $\mathcal{R}$.f

$$
\phi(t, x)=f\left(x_{1}+t \alpha_{1}(t, x), \ldots, x_{n}+t \alpha_{n}(t, x)\right) \dot{\epsilon} \mathcal{R} . f
$$

onde $x=\left(x_{1}, \ldots, x_{n}\right), t$ próximo de zero e $\alpha_{i}(t, 0)=0$. Derivando-o em relação ao tempo temos o vetor

$$
\left.\frac{\partial \phi}{\partial t}(t, x)\right|_{t=0}=\left(\alpha_{1} \frac{\partial f}{\partial x_{1}}, \ldots, \alpha_{n} \frac{\partial f}{\partial x_{n}}\right)
$$

onde $\alpha_{i}(0, x) \in \mathcal{M}_{n} \mathcal{E}_{n}$, logo temos que o $\mathcal{R}$-espaço tangente a $f$ é dado por

$$
L \mathcal{R} \cdot f=\mathcal{M}_{n}\left\{\frac{\partial f}{\partial x_{1}}, \ldots, \frac{\partial f}{\partial x_{n}}\right\} .
$$


Da mesma maneira, obtemos os espaços tangentes para os demais grupos de Mather, basta observar como é a ação do grupo sobre $\mathcal{M}_{n} \mathcal{E}(n, p)$ :

$$
\begin{aligned}
L \mathcal{L} \cdot f & =f^{*}\left(m_{p}\right) \cdot\left\{e_{1}, \cdots, e_{p}\right\} \\
L \mathcal{C} \cdot f & =f^{*}\left(m_{p}\right) m_{n}\left\{e_{1}, \cdots, e_{p}\right\} \\
L \mathcal{A} \cdot f & =L \mathcal{R} \cdot f+L \mathcal{L} \cdot f \\
L \mathcal{K} \cdot f & =L \mathcal{R} \cdot f+L \mathcal{C} \cdot f
\end{aligned}
$$

onde $e_{1}, \cdots, e_{p}$ são elementos da base canônica em $\mathbb{R}^{\mathbf{P}}$ (considerados como elementos de $\mathcal{E}(n, p)$ ).

Toda a construção acima foi feita para mudanças de coordenadas na fonte e na meta que preservam a origem. Se permitirmos que estas mudanças não fixem a origem, obtemos os espaços tangentes estendidos:

$$
\begin{aligned}
L \mathcal{R}_{e} \cdot f & =\mathcal{E}_{n} \cdot\left\{\frac{\partial f}{\partial x_{1}}, \cdots, \frac{\partial f}{\partial x_{n}}\right\} \\
L \mathcal{L}_{e} \cdot f & =f^{*}\left(\mathcal{E}_{p}\right) \cdot\left\{e_{1}, \cdots, e_{p}\right\} \\
L \mathcal{C}_{1} \cdot f & =f^{*}\left(\mathcal{E}_{p}\right) \cdot \mathcal{E}_{n}\left\{e_{1}, \cdots, e_{p}\right\} \\
L \mathcal{A}_{e} \cdot f & =L \mathcal{R}_{e} \cdot f+L \mathcal{L}_{e} \cdot f \\
L \mathcal{K}_{e} \cdot f & =L \mathcal{R}_{e} \cdot f+L \mathcal{C}_{e} \cdot f
\end{aligned}
$$

Definimos a $\mathcal{G}_{e}$-codimensão de um germe $f$ como segue:

$$
\mathcal{G}_{e}-\operatorname{codim}(f)=\operatorname{dim}_{\mathbb{R}} \frac{\mathcal{E}(n, p)}{L \mathcal{G}_{e} \cdot f}
$$

\subsubsection{Determinação finita}

Dado um germe $f$, existem mudanças de coordenadas de modo que este germe seja expresso de maneira "mais simples"? Ou ainda, de modo que $f$ seja $\mathcal{G}$-equivalente a seu polinômio de Taylor de grau $k$ para algum $k$ ?

Estas questões motivam a seguinte definição:

Definiçāo 1.1.1 Um germe $f \in \mathcal{M}_{n} \mathcal{E}(n, p)$ é dito $k-\mathcal{G}$ - determinado se qualquer $g \in$ $\mathcal{M}_{n} \mathcal{E}(n, p)$ tal que $j^{k} f=j^{k} g$ é $\mathcal{G}$ - equivalente a $f$.

Então um germe finitamente determinado é $\mathcal{G}$-equivalente ao seu polinômio de Taylor para algum grau $k$.

A investigação sobre o grau da determinação finita teve início com os trabalhos de Mather [25, $26,27,28,29]$ na década de 60 , onde temos uma estimativa grosseira do grau de determinação. Nos anos 70, Gaffney e du Plessis deram melhores aproximações (ver [39]). Apenas na década de 80, coin o trabalho de Bruce-du Plessis-Wall [8] o problema foi resolvido.

$O$ estudo da determinação finita começou com o objetivo de classificar os germes de aplicações, isto é, obter formas mais simples, porém equivalentes para expressar um dado germe. Os 
germes obtidos na classificação têm expressões mais simples, o que facilita o estudo dos mesmos. A determinação finita reduz o problema da classificação dos germes de aplicações ao espaço dos $k$-jatos, um espaço vetorial de dimensão finita.

Uma das técnicas utilizadas para a classificação é a indução sobre o espaço dos jatos. Dado um $j^{k} f$ verificamos se este é $k$-determinado, em caso afirmativo o processo pára, se não consideramos o $k+1$-jato dos germes cujo $k$-jato é igual a $j^{k} f$.

Uma das ferramentas fundamentais para a classificação é o Teorema da Transversal Completa apresentado por Bruce, Kirk e du Plessis [9].

Antes de enunciar o teorema vejamos uma definição.

Definição 1.1.2 Um conjunto $A$ é um espaço afim se existem um espaço vetorial $V_{A}$ e uma aplicaçāo $A \times V_{A} \rightarrow A,(x, v) \mapsto x+v$ tais que

(i) $x+0=x$ e $x+(u+v)=(x+u)+v \forall x \in A, u, v \in V_{A}$;

(ii) para qualquer $x, y \in A$ existe um único $v \in V_{A}$ tal que $y=x+v$.

Teorema 1.1.3 [9] Teorema da Transversal Completa Seja $G$ um grupo de Lie agindo suavemente sobre um espaço afim $A$, e seja $W$ um subespaço vetorial de $V_{A}$ com

$$
L G \cdot(x+w)=L G \cdot x, \forall x \in A e w \in W .
$$

Entāo

(i) $\forall x \in A$

$$
x+\{L G \cdot x \cap W\} \subset G . x \cap\{x+W\} .
$$

(ii) Se $x_{0} \in A$ e Té um subespaço vetorial de $W$ tal que

$$
W \subset T+L G \cdot x_{0}
$$

então $\forall w \in W$, existe $g \in G, t \in T$ tal que $g .\left(x_{0}+w\right)=x_{0}+t$.

$O$ subespaço $T$ é conhecido como Transversal Completa.

\subsubsection{Desdobramentos versais}

Seja $f: \mathbb{R}^{\mathrm{n}}, 0 \rightarrow \mathbb{R}^{\mathrm{p}}, 0$ um germe de aplicação diferenciável. Um desdobramento a s-parâmetros de $f$ é uma aplicação

$$
\begin{aligned}
F: \mathbb{R}^{\mathrm{n}} \times \mathbb{R}^{\mathrm{s}}, 0 & \rightarrow \mathbb{R}^{\mathrm{p}} \times \mathbb{R}^{\mathrm{s}}, 0 \\
(x, u) & \mapsto F(x, u)=(\bar{f}(x, u), u)
\end{aligned}
$$

tal que $\bar{f}(x, 0)=(f(x), 0)$. A aplicação $\bar{f}$ é chamada deformação de $f$.

Consideramos agora o grupo $\mathcal{A}$, mas os resultados são análogos para qualquer grupo de Mather. Dois desdobramentos $F, G: \mathbb{R}^{\mathrm{n}} \times \mathbb{R}^{\mathrm{s}}, 0 \rightarrow \mathbb{R}^{\mathrm{p}} \times \mathbb{R}^{\mathrm{s}}, 0$ de $f$ são isomorfos se existirem dois germes de difeomorfismos 


$$
\begin{aligned}
& \phi: \mathbb{R}^{\mathrm{n}} \times \mathbb{R}^{\mathrm{s}}, 0 \rightarrow \mathbb{R}^{\mathrm{n}} \times \mathbb{R}^{\mathrm{s}}, 0 \quad \mathrm{e} \\
& \psi: \mathbb{R}^{\mathrm{p}} \times \mathbb{R}^{\mathrm{s}}, 0 \rightarrow \mathbb{R}^{\mathrm{p}} \times \mathbb{R}^{\mathrm{s}}, 0
\end{aligned}
$$

que são desdobramentos a $s$-parâmetros dos germes da identidade em $\mathbb{R}^{\mathrm{n}}$ e $\mathbb{R}^{\mathrm{p}}$ respectivamente, tal que

$$
G=\psi \circ F \circ \phi^{-1} \text {. }
$$

Dentre os desdobramentos de $f$, destacam-se os desdobramentos versais. Dizemos que um desdobramento $F$ do germe $f$ é versal se qualquer outro desdobramento a $r$-parâmetros $G$ desse germe é induzido por $F$, ou seja, existe um germe $h: \mathbb{R}^{\mathbf{r}}, 0 \rightarrow \mathbb{R}^{\mathrm{s}}, 0$ tal que $G$ é isomorfo a $h^{*} F$, onde $h^{*} F(x, u)=(\vec{f}(x, h(u)), u)$.

Temos uma caracterização algébrica dos desdobramentos versais pelo seguinte teorema devido à Martinet, aqui enunciado para o grupo $\mathcal{A}$, porém válido para todos os grupos de Mather. Teorema 1.1.4 [24] $F: \mathbb{R}^{\mathrm{n}} \times \mathbb{R}^{\mathrm{s}}, 0 \rightarrow \mathbb{R}^{\mathrm{p}}, 0$ é um desdobramento versal do germe $f$ se, $e$ somente se,

$$
T \mathcal{A}_{e} f+\mathbb{R} \cdot\left\{\bar{f}_{i}\right\}=\mathcal{E}(n, p)
$$

onde $\overline{f_{i}}=\frac{\partial \bar{f}}{\partial u_{i}}(x, 0)$ é a velocidade inicial da deformação $\bar{f}, i=1, \ldots, s$.

Sendo assim, o número mínimo de parâmetros em um desdobramento versal de $f$ é igual a $\mathcal{A}_{e}$-codimensão de $f$.

Dado um desdobramento $F$ do germe $f$, diremos que $F$ é trivial se é isomorfo ao desdobramento constante $(x, u) \rightarrow(\bar{f}(x), u)$. Donde temos a seguinte definição. Um germe $f$ é estável se todos os desdobramentos de $f$ forem triviais. Como conseqüência desta definição temos que um germe $f$ é estável se, e somente se, sua codimensão é nula.

Dado um desdobramento versal $F$ de um germe $f$ podemos obter informações geométricas sobre $f$ através de alguns conjuntos associados a esse desdobramento, tais como o conjunto dos pontos críticos, o discriminante e o conjunto de bifurcação.

O conjunto dos pontos críticos de $F$ é definido por

$$
\Sigma F=\left\{(x, u): D F_{x}(x, u) \text { é singular }\right\}
$$

À imagem do conjunto dos pontos críticos pela $F, \Delta(F)=F(\Sigma F)$, chamamos discriminante.

O conjunto de bifurcação é definido por

$$
B i f(F)=\left\{u \in \mathbb{R}^{\mathrm{s}}: \exists x \in \mathbb{R}^{\mathrm{n}}, 0 \text { com } f_{u} \text { instável em } x\right\}
$$

Um dos resultados essenciais para a aplicação da teoria de desdobramentos versais é o seguinte:

Teorema 1.1.5 ([24]) Quaisquer dois desdobramentos versais com mesmo número de parâmetros têm conjuntos críticos, discriminantes e conjuntos de bifurcaçäo difeomorfos. 


\subsection{O subgrupo $\mathcal{A}^{*}$}

Dado um par de 1-formas diferenciais $(\alpha, \beta)$ numa vizinhança de um ponto $p \in M \subset \mathbb{R}^{2}$, podemos considerar cartas locais de modo que essa vizinhança do ponto $p \in M$ seja a imagem de um aberto $U \subset \mathbb{R}^{2}, 0$ por uma carta $\phi: U \rightarrow M$ com $\phi(0,0)=p$. Dessa maneira podemos considerar $M$ localmente como o plano. Além disso, se $\alpha$ é germe de 1-forma regular podemos supor que $\alpha=d y$, através do Teorema da Retificação [2]. Se $\beta$ também é regular, existe um germe de função regular $f: \mathbb{R}^{2}, 0 \rightarrow \mathbb{R}, 0$ tal que $\beta=d f$. Sendo assim, classificar os pares $(\alpha, \beta)$ é equivalente a classificar germes de funçōes regulares $f$, através de mudanças de coordenadas que preservem as folhas do germe de 1-forma $d y$ (as retas horizontais $y=c \in \mathbb{R}, 0$ ) na fonte $\mathrm{e}$ qualquer mudança de coordenadas na meta.

Portanto as mudanças de coordenadas na fonte são da forma

$$
h(x, y)=\left(h_{1}(x, y), h_{2}(y)\right)
$$

Essas mudanças de coordenadas formam um subgrupo do grupo $\mathcal{A}$, que denotamos por $\mathcal{A}^{*}$. O grupo $\mathcal{A}^{*}$ é dado por $\mathcal{A}^{*}=\mathcal{R}^{*} \times \mathcal{L}$, onde $\mathcal{R}^{*}$ é o grupo dos germes de difeomorfismos $h: \mathbb{R}^{2}, 0 \rightarrow \mathbb{R}^{2}, 0$ que preservam as retas $y=$ constante, isto é, $h(x, y)=\left(h_{1}(x, y), h_{2}(y)\right)$. Desde que $h$ é difeomofismo local, temos

$$
\frac{\partial h_{1}}{\partial x}(0,0) h_{2}^{\prime}(0) \neq 0
$$

A ação de $\mathcal{A}^{*}$ sobre $\mathcal{M}_{2}$ é a ação do grupo $\mathcal{A}$ restrita a $\mathcal{A}^{*}$.

Usaremos a notação $\mathcal{M}_{y}$ para denotar as funçōes que se anulam na origem e dependem apenas da variável $y$, ressaltando assim qual variável está sendo considerada em $\mathbb{R}^{2}$.

Para a classificação precisamos ainda descrever o espaço tangente às órbitas de $\mathcal{A}^{*}$ em $f$ : $\mathbb{R}^{2}, 0 \rightarrow \mathbb{R}, 0$. Desde que as mudanças de coordenadas na meta são arbitrárias o $\mathcal{L}$-espaço tangente é dado por $L \mathcal{L} \cdot f$. Quanto a fonte, temos que o conjunto dos campos de vetores tangentes a $\mathcal{R}^{*}$-órbita de $f$ é $L \mathcal{R}^{*} \cdot f$, dado por $L \mathcal{R}^{*} \cdot f=\mathcal{M}_{2}\left\{f_{x}\right\}+\mathcal{M}_{y}\left\{f_{y}\right\}$. Portanto

$$
L \mathcal{A}^{*} f=\mathcal{M}_{2}\left\{f_{x}\right\}+\mathcal{M}_{y}\left\{f_{y}\right\}+f^{*}\left(\mathcal{M}_{u}\right)
$$

onde $f_{x}$ e $f_{y}$ são as derivadas parciais de $f$ em relação a $x$ e $y$ respectivamente.

Uma questão natural é se ainda temos um teorema sobre determinação finita e desdobramentos versais para o grupo $\mathcal{A}^{*}$. A questão foi respondida pelos estudos de Damon [15]. Damon demonstrou que existem subgrupos dos grupos $\mathcal{A}$ e $\mathcal{K}$ para os quais existem teoremas da determinação finita e do desdobramento versal, chamou-os de subgrupos geométricos. Vamos verificar que o grupo $\mathcal{A}^{*}$ satisfaz a definição dada por Damon aos subgrupos geométricos. 


\subsubsection{O subgrupo geométrico $\mathcal{A}^{*}$ e suas propriedades}

Primeiramente vamos estudar as propriedades que definem os subgrupos geométricos e em seguida verificar que $\mathcal{A}^{*}$ satisfaz tais propriedades. Para isso algumas definições são necessárias.

Definição 1.2.1 Seja $\Phi: R \rightarrow S$ um homomorfismo de anéis. $N$ um $R$-módulo e $M$ um $S$-módulo, então $\alpha: N \rightarrow M$ é um homomorfismo de módulos sobre $\Phi$ se satisfaz $\alpha(r . n)=$ $\Phi(r) . \alpha(n), \forall r \in R$ e $n \in N$.

Definiçāo 1.2.2 [22] Uma álgebra diferencial ou uma DA-álgebra consiste de uma IR-álgebra e um homomorfismo de álgebras sobrejetor $\psi: \mathcal{E}_{y} \rightarrow A$. Em geral, $A$ é um anel local com ideal maximal $\mathcal{M}_{A}$.

Dadas duas álgebras diferenciais $(A, \psi)$ e $(B, \phi)$, um homomorfismo de DA-álgebras é uma aplicação $\alpha: A \rightarrow B$ que levanta o diagrama comutativo abaixo

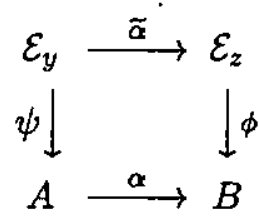

onde $\widetilde{\alpha}$ coincide com o pull-back do germe $g: \mathbb{R}^{\mathrm{m}}, 0 \rightarrow \mathbb{R}^{\mathrm{n}}, 0$.

Um sistema de anéis associados a um conjunto $D$ (finito e parcialmente ordenado) é um conjunto de anéis $\left\{R_{\alpha}: \alpha \in D\right\}$ e homomorfismos de anéis $\left\{\phi_{\alpha \beta}: R_{\alpha} \rightarrow R_{\beta}\right\}$ para $\alpha \leq \beta$, tal que $\phi_{\beta \gamma} \circ \phi_{\alpha \gamma}=\phi_{\alpha \gamma}$ para $\alpha \leq \beta \leq \gamma$ e $\phi_{\alpha \alpha}=i d$.

Notação: $\left\{R_{\alpha}\right\}$ denota um sistema de anéis.

Um sistema de anéis é dito sistema adequadamente ordenado de anéis se cada elemento de $D$ tem apenas um predecessor imediato.

Um sistema de $D A$-álgebras é um sistema de anéis $\left\{R_{\alpha}\right\}$ para os quais $R_{\alpha}$ é uma DA-álgebra e os homomorfismos de conexão são homomorfismos de DA-álgebras.

Exemplo: $\mathcal{E}_{(x, y)}, \mathcal{E}_{y}$ e $\mathcal{E}_{z}$ são anéis locais e podemos definir os homomorfismos de conexão por

$$
\mathcal{E}_{z} \stackrel{f^{*}}{\rightarrow} \mathcal{E}_{(x, y)} \stackrel{i^{*}}{\rightarrow} \mathcal{E}_{y}
$$

onde $f \in \mathcal{E}_{2}$ e $i: \mathbb{R}, 0 \rightarrow \mathbb{R}^{2}, 0$ é definida por $i(y)=(0, y)$. Além disso cada anel tem apenas um predecessor imediato, $\log \left\{\mathcal{E}_{(x, y)}, \mathcal{E}_{y}, \mathcal{E}_{z}\right\}$ formam um sistema adequadamente ordenado de anéis.

$\mathrm{Um}\left\{R_{(x, y, z)}\right\}$-módulo $M$ consiste de uma soma direta $M_{1} \oplus M_{2} \oplus M_{3}$ tal que $M_{1}$ é um $\mathcal{E}_{(x, y)}$-módulo e $M_{2}$ é um $\mathcal{E}_{y}$-módulo e $M_{3}$ é um $\mathcal{E}_{z}$-módulo.

Dizemos que $M$ é finitamente gerado se cada $M_{i}$ for um módulo finitamente gerado sobre o anel correspondente, $1 \leq i \leq 3$. 
$\mathrm{Um}\left\{R_{(x, y, z)}\right\}$ homomorfismo de módulos $\psi: M \rightarrow N$ consiste de uma soma de homomorfismos $\psi_{i j}: M_{j} \rightarrow N_{i}$ para $1 \leq j \leq i \leq 3$ os quais são homomorfismos sobre os homomorfismos de conexão $\phi_{i j}^{*}$.

Exemplo: Para $\mathcal{A}^{*}$ temos $M=T_{e} \mathcal{A}^{*}=\mathcal{E}_{(x, y)}\left\{\frac{\partial}{\partial x}\right\} \oplus \mathcal{E}_{y}\left\{\frac{\partial}{\partial y}\right\} \oplus \mathcal{E}_{z}\left\{\frac{\partial}{\partial z}\right\}$ e dof $: T \mathcal{A}_{e}^{*} \rightarrow$ $\mathcal{E}_{(x, y)}\left\{\frac{\partial}{\partial z}\right\}$ que é um homomorfismo de módulos:

$d \alpha_{f} \mid \mathcal{E}_{(x, y)}$ é um homomorfismo de $\mathcal{E}_{(x, y)}$-módulos. Defina $\frac{\partial}{\partial x} \mapsto \frac{\partial f}{\partial x}$ e $\phi(h)=h, \forall h \in \mathcal{E}_{(x, y)}$.

$d \alpha_{f} \mid \mathcal{E}_{y}$ é homomorfismo de $\mathcal{E}_{y}$-módulo, definindo $\frac{\partial}{\partial y} \mapsto \frac{\partial f}{\partial y}$ e $\phi(s)=s$.

$d \alpha_{f} \mid \mathcal{E}_{z}$ é homomorfismo de $\mathcal{E}_{z}$-módulo, se $\frac{\partial}{\partial z} \mapsto 1$ e $\phi(r)=f^{*}(r)$.

$N$ é um $\left\{R_{(x, y, z)}\right\}$-submódulo de $M$ se $N=N_{1} \oplus N_{2} \oplus N_{3}$, onde $N_{i}$ é submódulo de $M_{i}$ para $1 \leq i \leq 3$.

Se $\left\{\mathcal{I}_{(x, y, z)}\right\}=\left\{\mathcal{I}_{(x, y)}, \mathcal{I}_{y}, \mathcal{I}_{z}\right\}$ é um sistema de ideais de $\left\{R_{(x, y, z)}\right\}$, definimos $\left\{\mathcal{I}_{(x, y, z)}\right\} M=$ $\mathcal{I}_{(x, y)} M_{1} \oplus \mathcal{I}_{y} M_{2} \oplus \mathcal{I}_{z} M_{3}$

Observe que cada anel em $\left\{R_{(x, y, z)}\right\}$ é uma $\mathcal{E}_{z}$-álgebra. $\left\{R_{(x, y, z)}\right\}$ é um sistema adequadamente ordenado de DA-álgebras sobre $\mathcal{E}_{z}$ se cada homomorfismo de conexão é um homomorfismo de $\mathcal{E}_{z}$-álgebras e cada anel tem exatamente um predecessor.

Observação: Os homomorfismos de conexão para um sistema de anéis $\left\{R_{x}\right\}$ dependem de certos germes. Contudo, a estrutura de $\left\{R_{x}\right\}$-módulos não dependem desses homomorfismos, o que torna possível especificar um $\left\{R_{x}\right\}$-módulo sem especificar os homomorfismos de conexão. Mas para homomorfismos de $\left\{R_{x}\right\}$-módulos estes homomorfismo precisam ser especificados.

Definição 1.2.3 Seja $F$ um desdobramento de $f_{0} \in \mathcal{E}_{2}$. Então $\mathcal{A}_{u n}(k)=\left\{(\phi, \psi) \in \mathcal{D}_{2+k} \times\right.$ $\mathcal{D}_{1+k}:(\phi, \psi)$ são desdobramentos à $k$ parâmetros de $\left.\left(\phi_{0}, \psi_{0}\right) \in \mathcal{A}\right\}$, isto é, $(\phi, \psi) \cdot F$ é desdobramento à $k$-parâmetros de $\left(\phi_{0}, \psi_{0}\right) \cdot f_{0}$.

Dizemos que dois desdobramentos $F$ e $G$ à $r$-parâmetros são $\mathcal{A}$-equivalentes se existem $(\phi, \psi) \in \mathcal{A}_{\text {un }}$ e um difeomorfismo $\lambda: \mathbb{R}^{\mathrm{n}} \rightarrow \mathbb{R}^{\mathrm{r}}$ tal que $\psi \circ F \circ \phi^{-1}=\lambda^{*} G$. Isto define uma relação em $\mathcal{A}_{u n}$. O conjunto de todos os desdobramentos equivalentes formam um grupo que denotamos por $\mathcal{A}_{e q}(r)$.

Definição 1.2.4 Um grupo $\mathcal{G}$ agindo em $\mathcal{F}$ (junto com o grupo de desdobramento correspondente) será um subgrupo geométrico de $\mathcal{A}$ se satisfizer as quatro seguintes propriedades:

- naturalidade;

- estrutura de espaço tangente;

- aplicação exponencial;

- condição de filtração. 
Vejamos o que significa cada uma dessas propriedades:

Naturalidade: o grupo e o espaço de desdobramentos são naturais com relação ao pull-back. É possível considerar elementos de $\mathcal{G}_{u n}(q+1)$ e $\mathcal{F}_{u n}(q+1)$ como desdobramentos a 1-parâmetro de elementos de $\mathcal{G}_{u n}(q)$ e $\mathcal{F}_{\text {un }}(q)$ via pull-back pela imersão $\mathbb{R}^{\mathrm{q}} \rightarrow \mathbb{R}^{\mathrm{q}+1}$.

Estrutura do espaço tangente: Descreve a estrutura algébrica dos espaços tangentes e espaços tangentes estendidos e suas relações:

i) Existe uma coleção de DA-álgebras $\left\{R_{x}\right\}$ de $\mathcal{E}_{x}$ tal que para cada $\mathbb{R}^{\mathrm{q}}$ com coordenadas locais $\beta, L_{e} \mathcal{G}_{u n}(q)$ e $L_{e} \mathcal{F}_{u n}(q)$ são $\left\{R_{(x, \beta)}\right\}$-módulo finitamente gerados, com $L \mathcal{G}_{u n}(q)$ e $L \mathcal{F}_{u n}(q)$ $\left\{R_{(x, \beta)}\right\}$-submódulo finitamente gerados e, contendo (via naturalidade) $L_{e} \mathcal{G}$ e $L_{e} \mathcal{F}$ como $\left\{R_{x}\right\}$ submódulo.

ii) Para cada $F \in \mathcal{F}_{u n},\left\{R_{(x, \beta)}\right\}$ torna-se um sistema de DA-álgebras sobre $\mathcal{E}_{\beta}$ tal que

$$
d \mathcal{E}_{F}: L_{e} \mathcal{G}_{u n}(q) \rightarrow L_{e} \mathcal{F}_{u n}(q)
$$

é um homomorfismo de $\left\{R_{(x, \beta)}\right\}$-módulos.

iii) Requer que as aplicações naturais

$$
\frac{L_{e} \mathcal{G}_{u n}(q)}{\mathcal{M}_{\beta} L_{e} \mathcal{G}_{u n}(q)} \rightarrow L_{e} \mathcal{G} \quad \text { e } \frac{L_{e} \mathcal{F}_{u n}(q)}{\mathcal{M}_{\beta} L_{e} \mathcal{F}_{u n}(q)} \rightarrow L_{e} \mathcal{F}
$$

sejam isomorfismos de $\left\{R_{x}\right\}$-módulos.

iv) $\mathcal{M}_{x} L_{e} \mathcal{G} \subset L \mathcal{G}$ e $\mathcal{M}_{x} L_{e} \mathcal{F} \subset L \mathcal{F}$. Esta condição implica que os espaços tangentes estendidos diferem dos espaços tangentes por "campos de direçōes constantes".

Aplicação exponencial: $O$ espaço estendido ao grupo $\mathcal{G}_{e q}$ na identidade é dado por

$$
L_{e} \mathcal{G}_{e q}(q)=L_{e} \mathcal{G}_{u n}(q) \oplus \mathcal{E}_{\beta}\left\{\frac{\partial}{\partial \beta}\right\}
$$

A restrição da aplicação exponencial para $\mathcal{A}$ induz uma aplicação bem definida

$$
\begin{aligned}
\exp : L_{e} \mathcal{G}_{e q}(q) & \rightarrow \mathcal{G}_{e q}(q+1) \\
\xi & \mapsto \phi_{t}
\end{aligned}
$$

onde $\frac{\partial}{\partial t} \phi_{t}=\xi \cdot \phi_{t}$ e $\phi_{0}=i d$, sendo que $\phi_{t}(x, \lambda, y)=\left(\tilde{\phi}_{t}(x, \lambda), \psi(y, \lambda), h_{t}(\lambda)\right)$. Esta aplicação é um difeomorfismo para cada $t$, obtida pela integração do campo de direções $\xi$ (o fluxo).

Condição de Filtração: $\mathcal{G}_{u n}(q)$ preserva a filtração $\left\{\left\{\mathcal{M}_{x}^{l}\right\} \mathcal{F}_{u n}(q)\right\}$ sobre $\mathcal{F}_{u n}(q)$ e induz uma ação no quociente $\frac{\mathcal{F}_{u n}(q)}{\left\{\mathcal{M}_{x}^{l}\right\} \mathcal{F}_{u n}(q)}$, para todo $l \geq 0$

Para o nosso estudo, denotamos por $\mathcal{R}$ o grupo dos difeomorfismos em $\mathbb{R}^{2}, 0$. Lembremos que $\mathcal{A}=\{(\phi, \psi) \in \mathcal{R} \times \mathcal{L}\}$ é um grupo de difeomorfismos que age sobre $\mathcal{E}(2,1)$ da seguinte maneira: $(\phi, \psi) \cdot f=\psi \circ f \circ \phi^{-1}$. 
Como vimos anteriormente

$$
L \mathcal{A}^{*} \cdot f=\mathcal{M}_{x, y}\left\{\frac{\partial}{\partial x}\right\} \oplus \mathcal{M}_{y}\left\{\frac{\partial}{\partial y}\right\} \oplus f^{*}\left(\mathcal{M}_{z}\right),
$$

onde $z \in \mathbb{R}, 0$.

Conseqüentemente, para $\beta \in \mathbb{R}^{q}$ e $F \in \mathcal{E}(2+q, 1+q)$ temos

$$
L_{e} \mathcal{A}_{u n}^{*} \cdot F=\mathcal{E}_{x, y, \beta}\left\{\frac{\partial f}{\partial x}\right\} \oplus \mathcal{E}_{y, \beta}\left\{\frac{\partial f}{\partial y}\right\} \oplus F^{*}\left(\mathcal{E}_{z, \beta}\right) \oplus \mathcal{E}_{\beta}\left\{\frac{\partial}{\partial \beta_{i}}\right\}
$$

Vamos verificar que as quatro propriedades que caracterizam um subgrupo geométrico são válidas para o grupo $\mathcal{A}^{*}$.

1. Naturalidade pelo pull-back

Sejam $F: \mathbb{R}^{2+q} \rightarrow \mathbb{R}^{1+q}$ um desdobramento à $q$-parâmetros de $f: \mathbb{R} 62,0 \rightarrow \mathbb{R}, 0$ e $h: \mathbb{R}^{\mathrm{r}}, 0 \rightarrow \mathbb{R}^{\mathrm{q}}, 0$. Definimos o pull-back de $F$ por $h$ por $h^{*} F(x, v)=(\bar{f}(x, h(v)), v)$, onde $\bar{f}(x, 0)=f(x)$. Assim se $(\phi, \psi) \in \mathcal{A}_{u n}^{*}(q+1)$ e $i: \mathbb{R}^{\mathrm{q}}, 0 \rightarrow \mathbb{R}^{\mathrm{q}+1}, 0$ é a aplicação inclusão, temos

$$
\begin{aligned}
i^{*} \phi(x, y, \beta) & =\left(\phi_{1}(x, y, i(\beta)), \phi_{2}(y, i(\beta)), \beta\right), \\
i^{*} \psi(u, \beta) & =(\psi(u, i(\beta)), \beta)
\end{aligned}
$$

portanto $\left(i^{*} \phi, i^{*} \psi\right) \in \mathcal{A}_{u n}^{*}(q)$.

Esta propriedade para $\mathcal{F}_{\text {un }}=\mathcal{E}(2+q, 1+q)$, segue da definição.

2. Estrutura do espaço tangente

(i) Observe que $L \mathcal{A}_{u n}^{*} \cdot f$ é dado pela soma direta de módulos finitamente gerados sobre anéis locais. Na verdade isso motiva a definiçāo do seguinte sistema de anéis

$$
\left\{\mathcal{E}_{z} \stackrel{f^{*}}{\rightarrow} \mathcal{E}_{x, y} \stackrel{i^{*}}{\rightarrow} \mathcal{E}_{y}\right\}
$$

onde $f: \mathbb{R}^{2}, 0 \rightarrow \mathbb{R}, 0$ e $i: \mathbb{R}, 0 \rightarrow \mathbb{R}^{2}, 0$ é a aplicação inclusão. Já vimos que este é um sistema adequadamente ordenado de DA-álgebras em $\mathcal{E}_{x, y}$ com os homomorfismos de conexão apresentados acima. Denotamos este sistema de DA-álgebras por $\left\{R_{x, y}\right\}$. Logo para $\beta \in \mathbb{R}^{\mathrm{q}}, 0$, definimos o seguinte sistema de DA-álgebras

$$
\left\{\mathcal{E}_{\beta} \stackrel{\pi^{*}}{\rightarrow} \mathcal{E}_{z, \beta} \stackrel{f^{*}}{\rightarrow} \mathcal{E}_{x, y, \beta} \stackrel{i^{*}}{\rightarrow} \mathcal{E}_{y, \beta}\right\}
$$

$\operatorname{com} \pi: \mathbb{R}^{1+q}, 0 \rightarrow \mathbb{R}^{\mathrm{q}}, 0$ como a aplicação projeção natural. Denotamos este sistema por $\left\{R_{x, y, \beta}\right\}$.

Observe então que $L_{e} \mathcal{A}_{u n}^{*} \cdot f$ é um $\left\{R_{x, y, \beta}\right\}$-módulo finitamente gerado em $\mathcal{E}_{x, y}$ desde que é definido como soma direta de módulos finitamente gerados sobre este sistema de anéis (ver definição de $\left\{R_{x, y, \beta}\right\}$-módulo). Assim também $L_{e} \mathcal{E}_{2+q}=\mathcal{E}_{x, y, \beta}\left\{\frac{\partial}{\partial z}\right\}$ é um $\left\{R_{x, y, \beta}\right\}$-módulo cujos somandos com respeito aos anéis $\mathcal{E}_{\beta}, \mathcal{E}_{y, \beta}, \mathcal{E}_{z, \beta}$ são triviais.

Ainda mais, $L \mathcal{A}_{u n}^{*}$ e $L \mathcal{E}_{2+q}$ são $\left\{R_{x, y, \beta}\right\}$-submódulos finitamente gerados desde que, $\mathcal{M}_{x, y, \beta}$, $\mathcal{M}_{y, \beta}, \mathcal{M}_{z, \beta}, \mathcal{M}_{\beta}$ são ideais maximais dos anéis $\mathcal{E}_{x, y, \beta}^{*}, \mathcal{E}_{y, \beta}, \mathcal{E}_{z, \beta}, \mathcal{E}_{\beta}$, respectivamente. 
Note ainda que

$$
L_{e} \mathcal{A}^{*} \cdot f=\mathcal{E}_{x, y}\left\{\frac{\partial f}{\partial x}\right\} \oplus \mathcal{E}_{y}\left\{\frac{\partial f}{\partial y}\right\} \oplus f^{*}\left(\mathcal{E}_{z}\right)
$$

é um $\left\{R_{x, y}\right\}$-módulo finitamente gerado e contido em $L_{e} \mathcal{A}_{u n}^{*} \cdot f$ com a parcela referente a $\mathcal{E}_{\beta}$ trivial.

(ii) Seja $F \in \mathcal{F}_{u n}=\mathcal{E}(2+q, 1+q)$ e defina a aplicação órbita por $F$ dada por

$$
\begin{aligned}
\mathcal{E}_{F}: \mathcal{A}_{u n}^{*} & \rightarrow \mathcal{F}_{u n} \\
(\phi, \psi) & \mapsto \psi \circ F \circ \phi^{-1} .
\end{aligned}
$$

A "derivada" da aplicação $\mathcal{E}_{F}$ é dada pela aplicaçāo

$$
\begin{aligned}
L_{e} \mathcal{A}_{\text {un }}^{*}: & \rightarrow L_{e} \mathcal{E}(2+q, 1) \\
\left(\xi_{1}, \xi_{2}, \eta, \beta\right) & \mapsto \xi_{1}\left\{\frac{\partial F}{\partial x}\right\}+\xi_{2}\left\{\frac{\partial F}{\partial y}\right\}+F^{*}(\eta)+\beta\left\{\frac{\partial F}{\partial \beta_{i}}\right\} .
\end{aligned}
$$

Vamos então demonstrar que esta aplicação é um homomorfismo de $\left\{R_{x, y, \beta}\right\}$-módulos.

Defina os homomorfismos dos módulos da seguinte maneira:

$$
\begin{array}{llll}
\frac{\partial}{\partial x} \mapsto \frac{\partial F}{\partial x}, & \frac{\partial}{\partial y} \mapsto \frac{\partial F}{\partial y}, \\
\frac{\partial}{\partial z} \mapsto 1, & \frac{\partial}{\partial \beta_{i}} \mapsto \frac{\partial F}{\partial \beta_{i}},
\end{array}
$$

e os homomorfismos de anéis por

$$
\begin{array}{rrll}
\phi_{1}: & \mathcal{E}_{x, y, \beta} & \rightarrow \mathcal{E}_{x, y, \beta} & \text { identidade de } \mathcal{E}_{x, y, \beta} \\
\phi_{2}: & \mathcal{E}_{y, \beta} \rightarrow \mathcal{E}_{y, \beta} & \text { identidade de } \mathcal{E}_{y, \beta} \\
\phi_{3}: & \mathcal{E}_{z, \beta} \rightarrow \mathcal{E}_{z, \beta} & \text { pull back de } f \\
\phi_{4}: & \mathcal{E}_{\beta} \rightarrow \mathcal{E}_{\beta} & \text { identidade de } \mathcal{E}_{\beta}
\end{array}
$$

A partir das aplicações acima temos o seguinte diagrama:

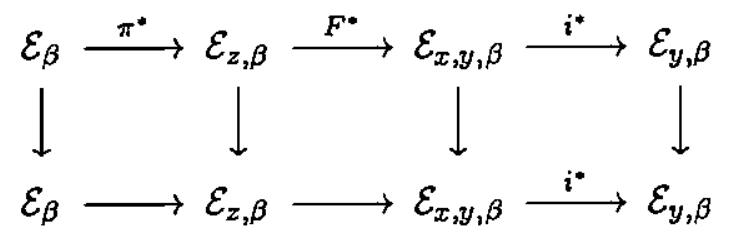

que é comutativo desde que os dois diagramas da direita são comutativos, em função de representarem o grupo $\mathcal{A}$ que é geométrico, e o último diagrama à esquerda é comutativo por construção.

Assim segue da definição de homomorfismo de $\left\{R_{\alpha}\right\}$-módulos que a aplicação $d \mathcal{E}_{F}$ é um homomorfismo de $\left\{R_{x, y, \beta}\right\}$-módulos.

(iii) Defina $\varphi: L_{e} \mathcal{A}_{u n}^{*}(q) \rightarrow L_{e} \mathcal{A}^{*}$ como a restrição da aplicação identidade a $\beta=0$. Então temos que

$$
\begin{aligned}
\xi(x, y, \beta)\left\{\frac{\partial}{\partial x}\right\}+\xi_{2}(y, \beta)\left\{\frac{\partial}{\partial y}\right\} & +\eta(z, \beta)\left\{\frac{\partial}{\partial z}\right\}+\tilde{\beta}(\beta)\left\{\frac{\partial}{\partial \beta_{i}}\right\} \mapsto \\
& \xi(x, y)\left\{\frac{\partial}{\partial x}\right\}+\xi_{2}(y)\left\{\frac{\partial}{\partial y}\right\}+\eta(z)\left\{\frac{\partial}{\partial z}\right\}
\end{aligned}
$$


sendo assim

$$
\begin{aligned}
\operatorname{ker}(\varphi) & =\left\{\left(\xi_{1}, \xi_{2}, \eta, \beta\right) \in L_{e} \mathcal{A}_{u n}^{*}: \xi_{1}(x, y, 0)=\xi_{2}(y, 0)=\eta(z, 0)=0\right\} \\
& =\mathcal{M}_{\beta} L_{e} \mathcal{A}_{u n}^{*} .
\end{aligned}
$$

Se aplicamos o Teorema do Isomorfismo temos que

$$
\frac{L_{e} \mathcal{A}_{u n}^{*}(q)}{\mathcal{M}_{\beta} L_{e} \mathcal{A}_{\text {in }}^{*}(q)} \rightarrow L_{e} \mathcal{A}^{*} \quad \text { e } \quad \frac{L_{e} \mathcal{E}_{2+q}}{\mathcal{M}_{\beta} L_{e} \mathcal{E}_{2+q}} \rightarrow L_{e} \mathcal{E}_{2}
$$

são isomorfismos de $\left\{R_{x, y}\right\}$-módulos, basta definir $\alpha=i d$ nas bases e os homormorfismos de anéis como os homomorfismos de conexão, veremos que o kernel será um sistema de ideais que irão gerar o $\operatorname{ker}(\varphi)$.

(iv) Observamos que dado o sistema de ideais maximais $\left\{\mathcal{M}_{x, y}\right\}=\left\{\mathcal{M}_{x, y}, \mathcal{M}_{y}, \mathcal{M}_{z}\right\}$ temos as seguintes igualdades:

$$
\begin{aligned}
\left\{\mathcal{M}_{x, y}\right\} \cdot L_{e} \mathcal{A}^{*} & =\mathcal{M}_{x, y}\left\{\frac{\partial}{\partial x}\right\}+\mathcal{M}_{y}\left\{\frac{\partial}{\partial y}\right\}+\mathcal{M}_{z}\left\{\frac{\partial}{\partial z}\right\}=L \mathcal{A}^{*} \\
\left\{\mathcal{M}_{x, y}\right\} \cdot L_{e} \mathcal{E}_{2} & =L \mathcal{E}_{2} .
\end{aligned}
$$

Com isso temos a condição sobre a estrutura do espaço tangente satisfeita para o grupo $\mathcal{A}^{*}$.

3. Aplicação exponencial

Considerando a restrição da aplicação exponencial definida no espaço tangente estendido ao grupo $\mathcal{A}_{\text {un }}$ para o grupo $\mathcal{A}_{u n}^{*}$ temos

$$
\begin{aligned}
\exp : L_{e} \mathcal{A}_{u n}^{*}(q) & \rightarrow \mathcal{A}_{e q}(q+1) \\
\xi & \mapsto \phi_{t}
\end{aligned}
$$

onde $\phi_{t}$ é o difeomorfismo obtido pela integração do campo $\xi$, sendo que este é dado pela soma direta dos campos $\xi_{1}\left\{\frac{\partial}{\partial x}\right\}+\xi_{2}\left\{\frac{\partial}{\partial y}\right\}, \eta\left\{\frac{\partial}{\partial z}\right\}$ e $\beta\left\{\frac{\partial}{\partial \beta_{i}}\right\}$.

Esta aplicação está bem definida se dado $\xi \in L_{e} \mathcal{A}_{u n}^{*}(q)$ o difeomorfismo obtido pela integração de $\xi$ pertencer ao grupo $\mathcal{A}_{e q}^{*}(q+1)$. Mas o que sabemos é que a aplicação exponencial satisfaz

$$
\left\{\begin{aligned}
\phi_{0} & =i d \\
\frac{\partial \phi_{t}}{\partial t} & =\xi\left(\phi_{t}\right)
\end{aligned}\right.
$$

então se $\xi \in L_{e} \mathcal{A}_{u n}^{*}(q)$ temos $\xi=\xi(x, y, z, \beta)=\left(\xi_{1}(x, y, \beta), \xi_{2}(y, \beta), \eta(z, \beta), \beta\right)$. Além disso sabemos que $\phi_{t} \in \mathcal{A}_{e q}(q+1)$, ou seja, $\phi_{t}(x, y, z, \beta)=\left(\tilde{\phi}_{t}^{1}(x, y, \beta), \tilde{\phi}_{t}^{2}(x, y, \beta), \psi_{t}(z, \beta), \tilde{\beta}_{t}\right)$, resta apenas mostrarmos que $\tilde{\phi}_{t}^{2}=\tilde{\phi}_{t}^{2}(y, \beta)$, ou seja, $\phi_{t}$ preserva as curvas $y=c \in \mathbb{R}, 0$, para concluir que $\phi_{t} \in \mathcal{A}_{e q}^{*}(q+1)$.

Observe no entanto que $\tilde{\phi}_{t}=\left(\tilde{\phi}_{t}^{1}, \tilde{\phi}_{t}^{2}\right)$ é solução do seguinte sistema:

$$
\begin{aligned}
& x^{\prime}=\xi_{1}(x, y) \\
& y^{\prime}=\xi_{2}(y)
\end{aligned}
$$


numa vizinhança da origem, isto é, satisfazendo $x(0)=x_{0}$ e $y(0)=y_{0}$. Então pelo teorema de existência e unicidade de soluções temos $y(t)=\varphi_{t}\left(t, y_{0}\right)$, ou seja, $\phi_{t}: \mathbb{R}^{2} \rightarrow \mathbb{R}^{2}$ com $t$ e $y_{0}$ fixos leva $y=c$ em $y=c^{\prime}$.

O que faz com que a aplicação exponencial esteja bem definida para o grupo $\mathcal{A}^{*}$.

4. Condição sobre a filtração

$\mathcal{A}_{u n}^{*}(q)$ preserva a filtração $\left\{\left\{\mathcal{M}_{x, y}\right\} \cdot \mathcal{E}_{2+q}\right\}$ sobre $\mathcal{F}_{u n}(q)=\mathcal{E}_{2+q}$ e induz a ação natural sobre o quociente

$$
\frac{\mathcal{E}_{2+q}}{\left.\left\{\mathcal{M}_{x, y}^{l}\right\} \cdot \mathcal{E}_{2+q}\right)}
$$

para todo $l \geq 0$, afinal a filtração acima nos dá a seguinte seqüencia:

$$
\mathcal{E}_{2+q} \supset \mathcal{M}_{2+q} \supset \ldots \supset \mathcal{M}_{2+q}^{k} \supset \ldots
$$

Então dado um germe $F \in \mathcal{M}_{2+q}^{k}$ para algum $k \geq 0$ e $(\phi, \psi) \in \mathcal{A}^{*}$ o germe $g=\psi \circ f \circ \phi^{-1} \in$ $\mathcal{M}_{2+q}^{k}$, como conseqüência da construção das mudanças de coordenadas para o grupo $\mathcal{A}$, o grupo $\mathcal{A}$ preserva o grau dos germes de aplicação, portanto $\mathcal{A}^{*}$ também.

Portanto as quatro propriedades que definem um subgrupo geométrico se verificam para o grupo $\mathcal{A}^{*}$. Isto é, $\mathcal{A}^{*}$ é um subgrupo geométrico de $\mathcal{A}$. Portanto temos assegurado por Damon, a validade dos teoremas da determinação finita e dos desdobramento versais para este grupo e - Teorema da Transversal Completa. Tudo isso nos dá ferramentas para a $\mathcal{A}^{*}$ - classificação de germes $f: \mathbb{R}^{2}, 0 \rightarrow \mathbb{R}, 0$.

Resta-nos então estimar o grau de determinação do germe $f$. Para isso temos o seguinte teorema válido para um sistema de DA-álgebras, dado em [15].

Teorema 1.2.5 [15] Seja $f: \mathbb{R}^{2}, 0 \rightarrow \mathbb{R}, 0$ germe na origem satisfazendo

$$
\mathcal{M}_{2}^{k+1} \subset L \mathcal{A}^{*} f+\mathcal{M}_{2}^{p}
$$

onde $p$ é um polinômio que depende de $k$. Entäo $f$ é $k-\mathcal{A}^{*}$-determinado.

Como em geral, o $p$ do teorema acima é grande, vamos usar o Teorema da Transversal Completa para mostrar que todas as $s$-tranversais para $s>k$ são vazias e assim obter a inclusão acima para o $p$ desejado.

Observação 1.2.6 Vale observar que foi preciso classificar por mudanças em $\mathcal{A}^{*}$ em vez de $\mathcal{K}^{*}=\mathcal{R}^{*} \times \mathcal{C}$. O grupo $\mathcal{K}^{*}$ classifica os germes de acordo com o seu contato na origem, independente do comportamento fora dela. Assim torna equivalentes germes que apesar de mesmo contato na origem têm comportamento distinto fora dela. Isso pode ser observado no exemplo abaixo. 
Exemplo: Considere os germes de funçöes $f(x, y)=y-x^{3}$ e $g(x, y)=y+x y+x^{3}$. As curvas de nível de ambos os germes possuem contato $3 \mathrm{com}$ as folhas de $\alpha=d y$ na origem e além disso é fácil verificar que $f$ e $g$ são germes $\mathcal{K}^{*}$-equivalentes. Mas se consideramos uma vizinhança da origem veremos que o discriminante (veja Definição 1.4.1) do par $\left(d y, d\left(y-x^{3}\right)\right.$ ) é dado por $\left\{x^{2}=0\right\}$, onde o contato é sempre 3, enquanto que o discriminante do par $\left(d y, d\left(y+x y+x^{3}\right)\right)$ é uma parábola, onde com a exceção da origem, o contato é dois. Queremos classificar pares de 1-formas de modo que fatos como esse signifiquem que os pares não são equivalentes. Veremos que os germes $y+x y+x^{3}$ e $y+x^{3}$ não são $\mathcal{A}^{*}$-equivalentes, sendo que o primeiro é $\mathcal{A}^{*}$-finitamente determinado e o segundo não.

\section{3 $\mathcal{A}^{*}$-classificação de germes regulares $\mathbb{R}^{2}, 0 \rightarrow \mathbb{R}, 0$}

Os germes $\mathbb{R}^{2}, 0 \rightarrow \mathbb{R}, 0$ em consideração são os germes de submersões. O principal resultado deste capítulo se resume no seguinte teorema:

Teorema 1.3.1 Os únicos germes $\mathcal{A}^{*}$-finitamente determinados estäo descritos na tabela abaixo, onde exibimos a $\mathcal{A}_{e}^{*}$-codimensão dos germes e o número máximo de singularidades $3_{1}$ que aparecem em uma deformação genérica do germe (ver seção 1.6).

\begin{tabular}{|c|l|c|c|}
\hline nome & modelo & $\mathcal{A}^{*}$-codimensão & $\sharp 3_{1}$ \\
\hline 1 & $x$ & 0 & 0 \\
2 & $y+x^{2}$ & 0 & 0 \\
$3_{2 p+1}$ & $y+x y^{2 p+1}+x^{3}, p \geq 0$ & $2 p$ & $2 p+1$ \\
$3_{2}$ & $y \pm x y^{2}+x^{3}$ & 1 & 2 \\
$3_{2 p}$ & $y \pm x y^{2 p}+\lambda x y^{5 p-1}+x^{3}, p \geq 2$ & $2 p$ & $2 p$ \\
\hline
\end{tabular}

A demonstração do Teorema 1.3.1 é dada neste capítulo como conseqüência de algumas proposiçōes que seguem abaixo.

Lema 1.3.2 Lema Preparatório Seja $f: \mathbb{R}^{2}, 0 \rightarrow \mathbb{R}, 0$ germe de aplicação diferenciável na origem, com $j^{1} f(0,0) \neq 0$. Se $f^{-1}(0)$ é uma curva que tem contato $k+1$ com a reta $y=0$, então existem mudanças de coordenadas em $\mathcal{A}^{*}$ tal que $f$ pode ser escrita na forma

$$
f(x, y)=x^{k+1}+h_{k-1}(y) x^{k-1}+\ldots+h_{1}(y) x+y
$$

para alguns germes de funções $h_{i} \in \mathcal{M}_{y}$, com $i=1, \ldots, k-1$.

Demonstração: Desde que $f^{-1}(0)$ tem contato $k+1$ com a curva $y=0$, podemos escrever $f(x, 0)= \pm x^{k+1}+$ t.o.m, ou seja, $f(x, 0) \sim_{\mathcal{R}} \pm x^{k+1}$. 
Um desdobramento $\mathcal{R}$-versal da singularidade $\pm x^{k+1}$ é dado por

$$
F\left(x, u_{k-1}, \ldots, u_{0}\right)=x^{k+1}+u_{k-1} x^{k-1}+\ldots+u_{1} x+u_{0}
$$

enquanto que a própria $f$ é um desdobramento a 1-parâmetro de $\pm x^{k+1}$, onde o parâmetro é a variável $y$. Logo existe $h: \mathbb{R}, 0 \rightarrow \mathbb{R}^{\mathrm{k}}, 0$, germe de aplicação diferenciável, tal que $f$ é isomorfa a $h^{*} F$, isto é,

$$
\begin{aligned}
f(x, y) & =h^{*} F(\psi(x, y), y)=F(\psi(x, y), h(y)) \\
& = \pm \psi^{k+1}(x, y)+h_{k-1}(y) \psi^{k-1}(x, y)+\ldots+h_{1}(y) \psi(x, y)+h_{0}(y)
\end{aligned}
$$

para algum germe $\psi: \mathbb{R}^{2}, 0 \rightarrow \mathbb{R}$. Como $(\psi(x, y), y)$ é difeomorfismo temos $\frac{\partial \psi}{\partial x}(0,0) \neq 0$. Além disso, por hipótese, $j^{1} f(0,0) \neq 0$, mas $\frac{\partial f}{\partial x}(0,0)=0$, logo $\frac{\partial f}{\partial y}(0,0)=h_{0}^{\prime}(0) \neq 0$. Portanto $\left(\psi, h_{0}\right) \in \mathcal{A}^{*}$. As mudanças de coordenadas $X=\psi(x, y), Y=h_{0}(y)$ e a multiplicação por \pm 1 (que é a mudança $\pm i d$ em $\mathcal{L}$ ), reduzem o germe $f$ na forma descrita acima.

Vamos então classificar $f$ de acordo com o contato da curva $f^{-1}(0)$ com $y=0$, como no Lema 1.3.2. Diremos contato de $f$ na origem com $y=0$ em vez do contato da curva $f^{-1}(0)$ com a curva $y=0$. O nosso objetivo é classificar os germes de $\mathcal{A}_{e}^{*}$-codimensão finita.

\subsubsection{Classificaçāo de germes com contato 1 e 2 na origem}

Proposição 1.3.3 Seja $f: \mathbb{R}^{2}, 0 \rightarrow \mathbb{R}, 0 \operatorname{com} j^{\mathrm{i}} f(0,0) \neq 0$.

(i) Se $f$ é transversal a reta $y=0$ na origem, então existe um difeomorfismo em $\mathcal{A}^{*}$ que leva $f$ ao germe $x$. Este é um germe $\mathcal{A}^{*}$-estável.

(ii) Se $f$ têm ordem de contato 2 na origem com a reta $y=0$, então $f$ é $\mathcal{A}^{*}$-equivalente a $y+x^{2}$, que é $\mathcal{A}^{*}$ estável.

Demonstração: (i) Do Lema Preparatório 1.3.2 temos $f$ equivalente a $g(x, y)=x+y$. Considerando a mudança de coordenadas $X=x+y, Y=y$ em $\mathcal{A}^{*}$ temos $f(x, y) \sim_{\mathcal{A}^{*}} x$. Então o germe $f$ é $1-\mathcal{A}^{*}$-determinado e estável. De fato para encontrarmos a $s$-transversal completa, para $s \geq 2$, observamos que $f_{x}=1$ e $f_{y}=0$, logo todas as transversais são vazias e temos $L \mathcal{A}^{*} \cdot f=\mathcal{M}_{2}$.

(ii) Se o contato de $f$ na origem com $y=0$ é igual a 2, pelo Lema Preparatório 1.3.2 temos o $f(x, y) \sim \mathcal{A}^{*} y+x^{2}$. Então a $s$-transversal completa, para $s \geq 3$, é vazia, afinal $f_{x}=2 x$ e $f_{y}=1$. Portanto o $\mathcal{A}^{*}$-espaço tangente a $f$ coincide com $\mathcal{M}_{2}$. Como no item (i) temos ainda um germe estável ( $\mathcal{A}_{e}^{*}$-codimensão zero). 


\subsubsection{Classificação de germes com contato 3 na origem}

Proposição 1.3.4 Seja $f: \mathbb{R}^{2}, 0 \rightarrow \mathbb{R}, 0$ tal que $j^{1} f(0,0) \neq 0$. Suponha que $f$ é de codimensão finita e têm contato 3 na origem com a reta $y=0$. Então existe um difeomorfismo levando o $f$ a um dos modelos abaixo:

$$
\begin{array}{ll}
3_{2 p+1}: & y+x y^{2 p+1}+x^{3}, p \geq 0 \\
3_{2}: & y \pm x y^{2}+x^{3} o u \\
3_{2 p}: & y+x\left( \pm y^{2 p}+\lambda y^{5 p-1}\right)+x^{3}, p \geq 2
\end{array}
$$

onde $\lambda$ é um módulo suave. Além disso, se $f(x, y)=y+x \psi(y)+x^{3}$ sendo que todas as derivadas de $\psi$ se anulam na origem, existe um difeomorfismo formal levando $f$ a $y+x^{3}$.

Uma deformação versal dos modelos acima são dados por

$$
\begin{aligned}
& F(x, y, u)=y+x\left(y^{2 p+1}+u_{2 p-1} y^{2 p-1}+\cdots+u_{0}\right)+x^{3} \\
& F(x, y, u)=y+x\left( \pm y^{2}+u_{0}\right)+x^{3} \\
& F(x, y, u)=y+x\left( \pm y^{2 p}+\left(\lambda+u_{2 p-1}\right) y^{5 p-1}+u_{2 p-2} y^{2 p-2}+\cdots+u_{0}\right)+x^{3} .
\end{aligned}
$$

Em particular, o germe $3_{1}$ é o caso estável.

Demonstração: Desde que $f$ têm contato 3 na origem com $y=0$, pelo Lema Preparatório 1.3.2 existe um sistema de coordenadas conveniente de modo que $f$ se escreve na forma

$$
f(x, y)=x^{3}+\psi(y) x+y
$$

Logo vamos classificar $f$ por indução sobre os jatos de $\psi$.

Suponha que $j^{k-1} \psi=0$ e $j^{k} \psi \neq 0$. Após mudanças escalares podemos escrever $j^{k} \psi(y)=$ $\pm y^{k}$. Considere o $(k+1)$-jato

$$
j^{k+1} f(x, y)=x^{3} \pm x y^{k}+y \text {. }
$$

que ainda denotamos por $f$. Portanto temos $f_{x}=3 x^{2} \pm y^{k}$ e $f_{y}=1 \pm k x y^{k-1}$. Vamos analisar 3 casos.

Caso 1 Se $k=1$, temos $f_{x}=3 x^{2}+y$ e $f_{y}=1+x$. Usando $f_{x}$ mostramos que todos os monômios de grau $s$, com exceção de $x^{s}$, serão gerados como produto de um polinômio de grau $s-1$ por $f_{x}$ em $L \mathcal{A}^{*} \cdot f+\mathcal{M}_{2}^{s+1}$. Para obter $x^{s}$, considere os seguintes vetores em $L \mathcal{A}^{*} \cdot f+\mathcal{M}_{2}^{s+1}$ :

$$
x^{s-2 j} y^{j-1} f_{x}=3 x^{s-2(j-1)} y^{j-1}+x^{s-2 j} y^{j}, \quad j \geq 1, \quad s-2 j \geq 0 .
$$

Então se $s$ é par $\left(s=2 j\right.$, para algum $j$ ), basta que mostremos que o monômio $y^{j}$ pertence a. $L \mathcal{A}^{*} \cdot f+\mathcal{M}_{2}^{s+1}$, se $s$ é ímpar $(s=2 j+1$, para algum $j$ ), precisamos mostrar que o monômio 
$x y^{j} \in L \mathcal{A}^{*} \cdot f+\mathcal{M}_{2}^{s+1}$. Em ambos os casos considere o sistema de vetores abaixo

$$
\begin{aligned}
x y^{j-1} f_{x} & =x y^{j}+3 x^{3} y^{j-1} \\
y^{j} f_{y} & =y^{j}+x y^{j} \\
f^{j} & =y^{j}+j x y^{j}+j x^{3} y^{j-1}+\text { t.o.m. }
\end{aligned}
$$

e mostre que todas as parcelas de $f^{j}$ pertencem a $L \mathcal{A}^{*} \cdot f+\mathcal{M}_{2}^{s+1}$, exceto $y^{j}, x y^{j}$ e $x^{3} y^{j-1}$. São elas: $y^{j}, y^{j-i}(x y)^{i}, y^{j-i} x^{3 i},(x y)^{j-i} x^{3 i}$, onde $1 \leq i \leq j$. Basta que $x^{i} y^{j}, x^{3 i} y^{j-i}$, com $2 \leq i \leq j \mathrm{e}$ $x^{2 i+j} y^{j-i}$, com $1 \leq i \leq j$ sejam vetores em $L \mathcal{A}^{*} \cdot f+\mathcal{M}_{2}^{s+1}$. Mas $2 i+j+j-i=2 j+i \geq s+1$, para $i \neq 1$. Para $i=1$ temos o monômio $x^{j+2} y^{j-1}$, então considere os vetores:

$$
x^{j+2 r} y^{j-r-1} f_{x}=3 x^{j+2 r+2} y^{j-r-1}+x^{j+2 r} y^{j-r}, \quad 1 \leq r \leq j .
$$

Fazendo $j=r$ temos $j+2 r=3 j \geq s+1$. Então $x^{2 i+j+1} y^{j-i}$ é vetor de $L \mathcal{A}^{*} \cdot f+\mathcal{M}_{2}^{k+1}$, para todo $j$.

Para as parcelas da forma $x^{i} y^{j}$ considere os vetores

$$
x^{i+2(r-1)} y^{j-\tau} f_{x}=3 x^{i+2 \tau} y^{j-\tau}+x^{i+2(r-1)} y^{j-r+1}, \quad 1 \leq r \leq j
$$

fazendo $j=r$ temos $x^{i+2 j-2} f_{x}=3 x^{i+2 j}+x^{i+2 j-2} y$. Como $i+2 j \geq s+i, i \geq 2$, temos $x^{i} y^{j} \in L \mathcal{A}^{*} \cdot f+\mathcal{M}_{2}^{s+1}$, com $2 \leq i \leq j$. Para as parcelas da forma $x^{3 i} y^{j-i}$ considere os vetores da forma

$$
x^{3 i+2(r-1)} y^{j-i-r} f_{x}=3 x^{3 i+2 r} y^{j-i-r}+x^{3 i+2(r-1)} y^{j-i-r+1}, \quad 1 \leq r \leq j-i .
$$

Fazendo $r=j-i$ temos

$$
x^{3 i+2 j-2 i-2} f_{x}=3 x^{3 i+2(j-i)}+x^{3 i+2 j-2-2 i} y .
$$

Como $3 i+2 j-2-2 i+1=i+2 j-1 \geq s+1$, com $i \geq 2$, temos que a inclusão desejada.

Portanto que a matriz associada ao sistema (1.9) é

$$
\left(\begin{array}{lll}
0 & 3 & 1 \\
1 & 0 & 1 \\
1 & j & j
\end{array}\right)
$$

cujo determinante é $(-3+4 j) \neq 0$. Concluímos assim que todos os monômios acima pertencem a $L \mathcal{A}^{*} \cdot f+\mathcal{M}_{2}^{s+1}$. Sendo assim temos $x^{s} \in L \mathcal{A}^{*} \cdot f+\mathcal{M}_{2}^{s+1}$.

Do estudo acima concluímos que as $(s+1)$-transversais completas são vazias, para todo $s \geq 4$. Então o germe $f(x, y)=x^{3}+x y+y$ é $3-\mathcal{A}^{*}$-determinado. Além disso o germe é também estável, desde que possui $\mathcal{A}_{e}^{*}$-codimensão nula.

Caso 2 Se $k=2$, temos $f_{x}=3 x^{2} \pm y^{2}$ e $f_{y}=1 \pm 2 x y$. Vamos mostrar que o germe $f$ é $3-\mathcal{A}^{*}$-determinado. 
Através do vetor $f_{x}$ temos duas formas de monômios a considerar: $x^{m-2 j} y^{2 j}$ e $x^{m-2 j+1} y^{2 j+1}$, onde $j \geq 0$ e $m \geq 4$. Vamos trata-los separadamente.

Os monômios da forma $x^{m-2 j} y^{2 j}$ são gerados pelos vetores

$$
x^{m-2 j} y^{2 j-2} f_{x}=3 x^{m-2 j+2} y^{2 j-2} \pm x^{m-2 j} y^{2 j}
$$

Se mostrarmos que o monômio $x^{m-2 j} y^{2 j}$ pertence a $L \mathcal{A}^{*} \cdot f+\mathcal{M}_{2}^{m+1}$ para algum $j$ teremos a inclusão para todos os demais monômios como conseqüência. Mas notemos que se $m$ é par, então existe $j$ tal que $m=2 j$, sendo assim temos:

$$
y^{m-2} f_{x}=3 x^{2} y^{m-2} \pm y^{m},
$$

logo basta considerar o vetor

$$
y^{m} f_{y}=y^{m} \pm 2 x y^{m+1}
$$

para concluir que $y^{m} \in L \mathcal{A}^{*} \cdot f+\mathcal{M}_{2}^{m+1}$. Logo $x^{m-2 j} y^{2 j} \in L \mathcal{A}^{*} \cdot f+\mathcal{M}_{2}^{m+1}$, para todo $j$.

Se $m$ é ímpar, existe $j$ tal que $m=2 j+1$, sendo assim temos

$$
x y^{m-3} f_{x}=3 x^{3} y^{m-3} \pm x y^{m-1} \text {. }
$$

Considere os vetores

$$
\begin{aligned}
y^{m-2} f_{y} & =y^{m-2} \pm 2 x y^{m-1} \\
f^{m-2} & =y^{m-2} \pm(m-2) x y^{m+1} \pm(m-2) x^{3} y^{m-1}+\text { t.o.m. }
\end{aligned}
$$

Observe que, com exceção de $y^{m-2}$, os demais monômios em $f^{m-2}$ pertencem a $\mathcal{M}_{2}$, logo $y^{m-2} \in L \mathcal{A}_{e}^{*} \cdot f+\mathcal{M}_{2}^{m+1}$, assim como os monômios da forma $x^{m-2 j} y^{2 j}$.

Quanto aos monômios da forma $x^{m-2 j+1} y^{2 j+1}$, eles são gerados pelos vetores

$$
x^{m-2 j+1} y^{2 j-1} f_{x}=3 x^{m-2 j+3} y^{2 j-1} \pm x^{m-2 j+1} y^{2 j+1}
$$

Daqui a análise segue de modo análogo a anterior.

Sendo assim temos que $f(x, y)=y \pm x y^{2}+x^{3}$ é $3-\mathcal{A}^{*}$-determinada. Quanto ao desdobramento versal de $f$, ele é dado como no enunciado da proposição, desde que utilizando um sistema semelhante ao encontrado acima, somos capazes de mostrar que o germe $f$ tem $\mathcal{A}_{e}^{*}$-codimensão 1 , pois $\mathcal{E}_{2} / L \mathcal{A}_{e}^{*} \cdot f=\mathbb{R} \cdot\{x\}$.

Caso 3 Se $k \geq 3$, temos $f_{x}=3 x^{2} \pm y^{k}$ e $f_{y}=1 \pm k x y^{k-1}$, logo todos os monômios divisíveis por $x^{2}$ e aqueles monômios da forma $y^{k+1+i}$ pertencem a $L \mathcal{A}^{*} \cdot f+\mathcal{M}_{2}^{k+2+i}$, para $i \geq 1$. Resta verificarmos se os monômios $x y^{k+i}$ também pertencem. Verifiquemos que as $(k+1+i)$ transversais completas são vazias. Então pelo Teorema 1.2.5 temos que o germe $f$ é $(k+1)-\mathcal{A}^{*}$ determinado. Para isso basta considerar os seguintes vetores:

$$
\begin{aligned}
x y^{i} f_{x} & =3 x^{3} y^{i}+x y^{k+i} \\
y^{i+1} f_{y} & =y^{i+1}+k x y^{k+i} \\
f^{i+1} & \sim \mathcal{A}^{*}
\end{aligned}
$$


As demais parcelas de $f^{i+1}$ pertencem a $L \mathcal{A}^{*} \cdot f+\mathcal{M}_{2}^{k+2+i}$, basta proceder como no caso $k=1$.

O determinante da matriz associada ao sistema acima é dado por $3 k-2(i+1)$. Logo não se anula se $k$ é ímpar. Anula-se para $k=2 p$ e $i=3 p-1$.

Sendo assim, quando $k=2 p+1$ o germe $y+x y^{2 p+1}+x^{3}$ é $(k+1)-\mathcal{A}^{*}$-determinado. Além disso, o germe tem $\mathcal{A}_{e}^{*}$ - codimensão $k-1$, pois $\mathcal{E}_{2} / L \mathcal{A}_{e}^{*} \cdot f=\mathbb{R} \cdot\left\{x, x y, \ldots, x y^{k-2}\right\}$. Então um desdobramento versal é dado como no enunciado do teorema.

Quando $k=2 p$, o determinante da matriz é não nulo, exceto para $i=3 p-1$, indicando que, um dos monômios do sistema acima não pertencem a $L \mathcal{A}^{*} \cdot f$. Digamos que seja o monômio $x y^{5 p-1}$. Concluímos então que a $(5 p-1)$-transversal é dada por $T=\mathbb{R} .\left\{x y^{5 p-1}\right\}$. Então $j^{5 p-1} f$ é equivalente a $y+x\left( \pm y^{2 p}+\lambda y^{5 p-1}\right)+x^{3}$, para algum $\lambda \in \mathbb{R}$. Vale observar que näo podemos eliminar $\lambda$ através de mudanças de coordenadas pertencentes a $\mathcal{A}^{*}$, logo $\lambda$ é um módulo suave.

Voltando a matriz associada ao sistema temos que todas as $s$-transversais com $s \geq 5 p+1$ são vazias, sendo assim $y \pm x\left(y^{2 p}+\lambda y^{5 p-1}\right)+x^{3}$ é $5 p-\mathcal{A}^{*}$-determinado para todo $\lambda \neq 0$. Além disso este germe tem codimensão $k$ (o estrato tem codimensão $k-1$ ), afinal $\mathcal{E}_{2} / L \mathcal{A}_{e}^{*} \cdot f=$ $\mathbb{R}:\left\{x, x y, \ldots, x y^{k-2}, x y^{5 p-1}\right\}$. Logo um desdobramento versal de $f$ é dado como no enunciado do teorema.

Passemos a estudar o caso em que as derivadas de todas as ordens de $\phi$ são nulas em 0 . Neste caso, $f(x, y)=x^{3}+\phi(y) x+y$ é formalmente equivalente ao germe $x^{3}+y$, pois $\forall l \geq 3$, $j^{l} f \simeq x^{3}+y$ e este germe não é $\mathcal{A}^{*}$-finitamente determinado.

\subsubsection{Contato $\geq 4$ na origem}

Vamos mostrar primeiramente que para contato $k=4$ não existe nenhum germe finitamente $\mathcal{A}^{*}$-determinado.

Supondo que $j^{1} f(0,0) \neq 0$, temos pelo Lema Preparatório 1.3.2 que nos permite escrever

$$
f(x, y)=y+x \psi_{2}(y)+x^{2} \psi_{1}(y)+x^{4}
$$

No caso menos degenerado $j^{1} \psi_{1}(y) \neq 0$ e $j^{1} \psi_{2}(y) \neq 0$. Uma mudança escalar reduz $j^{1}\left(\psi_{1}, \psi_{2}\right)=$ $(y ; \lambda y)$. Note que $\lambda$ não pode ser eliminado por mudanças em $\mathcal{A}^{*}$. Com isso temos a seguinte Proposição:

Proposição 1.3.5 Seja $f$ germe de função em $\mathbb{R}^{2}, 0$ dada por $f(x, y)=y+x \psi_{2}(y)+x^{2} \psi_{1}(y)+$ $x^{4}$, com $j^{1}\left(\psi_{1}, \psi_{2}\right)=(y, \lambda y)$. Entāo $f$ não é $\mathcal{A}^{*}$-finitamente determinado.

Demonstração: Vamos trabalhar nos níveis de $k$-jatos de $\left(\psi_{1}, \psi_{2}\right)$. Considerando o 1-jato, temos $f_{x}=y+2 \lambda x y+4 x^{3}$ e $f_{y}=1+x+\lambda x^{2}$. Fazendo $P_{k-1} f_{x}$, onde $P_{k-1}$ é um monômio de 
grau $k-1$, temos que todos os monômios de grau $k$ pertencem a $L \mathcal{A}^{*} \cdot f+\mathcal{M}_{2}^{k+1}$, exceto $x^{k}$. O que faremos então é verificar se $x^{k}$ pertence à $L \mathcal{A}^{*} \cdot f+\mathcal{M}_{2}^{k+1}$. Considere os vetores:

$$
x^{k-3 j} y^{j-1} f_{x}=4 x^{k-3 j+3} y^{j-1}+2 \lambda x^{k-3 j+1} y^{j}+x^{k-3 j} y^{j}, \quad j \geq 2, k-3 j \geq 0 .
$$

Nestas condições temos três possibilidades para $k: k=3 j, 3 j+1,3 j+2$, para algum $j$.

Caso 1: $k=3 j$. Neste caso obtemos o seguinte vetor $y^{j-1} f_{x}=y^{j}+2 \lambda x y^{j}+4 x^{3} y^{j-1}$. Considere então o vetor $y^{j} f_{y}=y^{j}+x y^{j}+\lambda x^{2} y^{j} \in L \mathcal{A}^{*} \cdot f+\mathcal{M}_{2}^{k+1}$. Vamos mostrar que os monômios da forma $x^{\tau} y^{j} \in L \mathcal{A}^{*} \cdot f+\mathcal{M}_{2}^{k+1}, \forall r \geq 1$, para isso considere os seguintes vetores neste espaço:

$$
x^{r+3 i} y^{j-1-i} f_{x}=x^{\tau+3 i} y^{j-i}+2 \lambda x^{\tau+3 i+1} y^{j-i}+4 x^{\tau+3 i+3} y^{j-i-1}, \quad 0 \leq i \leq j-1
$$

escolhendo $i=j-1$ e teremos

$$
x^{r+3 i} f_{x}=x^{r+3 i} y+2 \lambda x^{r+3 i+1} y+4 x^{r+3 i+3},
$$

desde que $r+3 i+3=r+3 j-3+3=r+3 j=k+r \geq k+1, x^{r+3 i+3} \in L \mathcal{A}^{*} \cdot f+\mathcal{M}_{2}^{k+1}$. $\mathrm{O}$ mesmo acontece ao monômio $x^{r+3 i+1} y$, pois $r+3 i+2=r+3 j-1=k+r-1 \geq k$, e todos os monômios de grau $k$ estão em $L \mathcal{A}^{*} \cdot f+\mathcal{M}_{2}^{k+1}$, exceto possivelmente $x^{k}$. Portanto o monômio $x^{r+3 i} y$ também pertence ao mesmo espaço tangente. Retornando através de (1.12), usando os passos acima teremos que os monômios da forma $x^{r} y^{j} \in L \mathcal{A}^{*} \cdot f+\mathcal{M}_{2}^{k+1}, \forall r \geq 1$. Logo do vetor $y^{j} f_{y}$ temos que o monômio $y^{j}$ também pertence a esse espaço, o que implica que $x^{3 j} \in L \mathcal{A}^{*} \cdot f+\mathcal{M}_{2}^{k+1}$ (através dos vetores em 1.11).

Caso 2: Se $k=3 j+1$ temos $x y^{j-1} f_{x}=x y^{j}+2 \lambda x^{2} y^{j}+4 x^{4} y^{j-1}$. Considere os vetores

$$
\begin{aligned}
y^{j} f_{y} & =y^{j}+x y^{j}+\lambda x^{2} y^{j} \\
f^{j} & =y^{j}+j x y^{j}+t . o . m . .
\end{aligned}
$$

Primeiramente verifiquemos que $f^{j} \simeq \mathcal{A}^{*} y^{j}+j x y^{j}+x^{4} y^{j}, y^{j} f_{y} \simeq \mathcal{A}^{*} y^{j}+x y^{j}$, e $x y^{j-1} f_{x} \simeq \mathcal{A}^{*}$ $x y^{j}+4 x^{4} y^{j-1}$.

Lembremos que as parcelas de $f^{j}$ são: $y^{j}, x^{i} y^{j}, x^{2 i} y^{j}, x^{j+i} y^{j}, x^{j+3 i} y^{j-i}, x^{2 i+2 j} y^{j-i}, x^{4 i} y^{j-i}$, com $1 \leq i \leq j$.

Seguindo os passos do Caso 1 , para $x^{r} y^{j}, r \geq 2$ temos os vetores em (1.12), considere $i=j-1$ e obtemos o vetor

$$
x^{r+3 j-3} f_{x}=x^{r+3 j-3} y+2 \lambda x^{r+3 j-2} y+4 x^{r+3 j} .
$$

Note que $r+3 j=r+k-1 \geq k+1, \forall r \geq 2$. Além disso os vetores da forma $x^{r+3 j-2} y$ pertencem a $L \mathcal{A}^{*} \cdot f+\mathcal{M}_{2}^{k+1}$, basta gerá-los na primeira parcela de $f_{x}$ assim como os monômios que aparecem nas demais parcelas, até que o grau dos monômios envolvidos na equação seja maior ou igual a $k+1$. Dessa forma os monônios $x^{i} y^{j}, x^{2 i} y^{j}, x^{j+i} y^{j}$ também pertencem a $L \mathcal{A}^{*} \cdot f+\mathcal{M}_{2}^{k+1}$. 
Em relação aos monômios da forma $x^{r} y^{j-i}$, como no caso anterior usaremos $f_{x}$ e a equaçāo (1.12), desta vez com $1 \leq i \leq j-1$. Pelo argumento feito no primeiro caso temos $r+3 j=$ $r+k-1 \geq k+1$, afinal $r \geq 2$ e lembrando que todos os monômios gerados na segunda parcela de $f_{x}$ pertencem a $L \mathcal{A}^{*} \cdot f+\mathcal{M}_{2}^{k+1}$ como feito no caso anterior, concluímos que $x^{r} y^{j-i} \in$ $L \mathcal{A}^{*} \cdot f+\mathcal{M}_{2}^{k+1}, \forall r \geq 2$.

Sendo assim temos as equivalências desejadas e podemos considerar o seguinte sistema de vetores:

$$
\begin{aligned}
x y^{j-1} f_{x} & =x y^{j}+4 x^{4} y^{j-1} \\
y^{j} f_{y} & \simeq \mathcal{A}^{*} \quad y^{j}+x y^{j} \\
f^{j} & \simeq \mathcal{A}^{*} \quad y^{j}+j x y^{j}+j x^{4} y^{j-1}
\end{aligned}
$$

cujo determinante da matriz associada é dado por $3 j-4 \neq 0$. Sendo assim temos que $x^{3 j+1} \in$ $L \mathcal{A}^{*} \cdot f+\mathcal{M}_{2}^{k+1}$, para todo $j \geq 2$.

Caso 3: $k=3 j+2$. Vamos mostrar que para todo $j \geq 2, x^{3 j+2} \notin L \mathcal{A}^{*} \cdot f+\mathcal{M}_{2}^{3 j+3}$. Portanto para todo $k$, existe $j$ de modo que $x^{3 j+2} \notin L \mathcal{A}^{*} \cdot f+\mathcal{M}_{2}^{k+1}$, isto é, o germe $f$ não é $\mathcal{A}^{*}$-finitamente determinado.

Considere o germe $f(x, y)=y+x y+\lambda_{1} x^{2} y+x^{4}+\lambda_{2} x^{8}+\lambda_{3} x^{11}+\ldots+\lambda_{n} x^{3 n+2}$, mostramos que comparando os monômios de grau menor ou igual a $3 m+2$, para $m>n$ como os vetores em $L \mathcal{A}^{*} \cdot f+\mathcal{M}_{2}^{3 m+3}$ envolvendo estes monômios, sempre teremos mais monômios do que vetores, logo existe monômio em $\mathcal{M}_{2}^{3 m+2}$ que nāo pertence a $L \mathcal{A}^{*} \cdot f+\mathcal{M}_{2}^{3 m+3}$.

Começamos pela lista dos monômios de grau menor ou igual a $3 m+2$ para $m>n$. Observamos que a partir de $f_{x}$ temos todos os monômios de grau $3 m+2$ em $L \mathcal{A}^{*} \cdot f+\mathcal{M}_{2}^{3 m+3}$, exceto $x^{3 m+2}$. Logo o monômio $x^{3 m-1} y$ também não pertence $L \mathcal{A}^{*} \cdot f+\mathcal{M}_{2}^{3 m+3}$, afinal $x^{3 m-1} f_{x}=$ $x^{3 m-1} y+2 \lambda x^{3 m} y+4 x^{3 m+2}+\ldots$, com $x^{3 m} y \in L \mathcal{A}^{*} \cdot f+\mathcal{M}_{2}^{3 m+3}$. Assim seguindo temos:

$\begin{array}{lllll}x^{k} & & & & \\ x^{k-1} & & & & \\ x^{k-2} & x^{k-3} y & & & \\ x^{k-3} & x^{k-4} y & & & \\ \ldots & \ldots & \ldots & & \\ x^{k-2 m} & x^{k-2 m-1} y & \ldots & x^{k-3 m} y^{m} & \\ x^{k-2 m-1} & x^{k-2 m-2} y & \ldots & x^{k-3 m-1} y^{m} & \\ x^{m} & x^{m-1} y & \ldots & x y^{m-1} & y^{m} \\ \ldots & \cdots & \ldots & \ldots & \ldots \\ x^{4} & x^{3} y & x^{2} y^{2} & x y^{3} & y^{4} \\ -- & x^{2} y & x y^{2} & y^{3} & \\ -- & x y & y^{2} & & \\ -- & y, & & & \end{array}$


onde $k=3 j+2$.

Observe que encontramos $\sum_{i=1}^{m+1} 2 i+\sum_{i=1}^{m}(i+1)-3$ monômios, ou seja, nosso sistema tem $\frac{3 m^{2}+9 m-2}{2}$ incógnitas (não consideramos os monômios $x^{3}, x^{2}, x$ porque eles não irão aparecem como parcelas nos vetores em $\left.L \mathcal{A}^{*} \cdot f+\mathcal{M}_{2}^{k+1}\right)$.

Vejamos então os vetores em $L \mathcal{A}^{*} \cdot f+\mathcal{M}_{2}^{3 m+3}$ envolvendo os monômios acima. O monômio $x^{r}$, para $4 \leq r \leq 3 m+2$ pode ser gerado por $x^{3 m-1} f_{x}, x^{3 m-2} f_{x}, \ldots, x f_{x}$. Analogamente ocorre com os monômios da forma $x^{r} y^{l}$, com $0 \leq r \leq 3 m-3 l+2$ para cada $l$ fixado em $1 \leq l \leq m$. Então temos os seguintes vetores a considerar :

$$
\begin{array}{lll}
x^{3 m-1} f_{x}, & \ldots & x f_{x} \\
x^{3 m-1-3 i} y^{i} f_{x}, & \ldots & y^{i} f_{x}, 2 \leq i \leq m-1 .
\end{array}
$$

Além dos vetores $y f_{y}, \ldots, y^{m} f_{y}$ e $f^{2}, \ldots, f^{m}$.

Portanto temos $\sum_{i=1}^{m-1} 3 i+5 m-2=\frac{3 m^{2}+7 m-4}{2}$ vetores.

Fazendo a diferença entre eles temos: VETORES - INCÓGNITAS $=\frac{3 m^{2}+7 m-4}{2}-\frac{3 m^{2}+9 m-2}{2}=$ $-(m+1)<0$. Ou seja, nosso sistema é impossível. Consequentemente $x^{3 m+2} \notin L \mathcal{A}^{*} \cdot f+\mathcal{M}_{2}^{3 m+3}$, para todo $m \geq 2$.

Concluímos portanto que o germe $f(x, y)=y+x y+\lambda x^{2} y+x^{4}$ não é $\mathcal{A}^{*}$-finitamente determinado.

Mostramos agora que não existe germe finitamente $\mathcal{A}^{*}$-determinado com contato 4 na origem, usando a proposição anterior e a que segue abaixo. A proposição a seguir é válida para todo subgrupo geométrico de $\mathcal{A}$ e $\mathcal{K}$ 'desde que neste caso temos garantida a existência de um teorema de determinação finita.

Proposição 1.3.6 Seja $\mathcal{G}$ subgrupo geométrico de $\mathcal{A}$ ou $\mathcal{K}, f: \mathbb{R}^{\mathrm{n}}, 0 \rightarrow \mathbb{R}^{\mathrm{p}}, 0$ germe finitamente $\mathcal{G}$-determinado e $F$ um desdobramento versal de $f$ com parâmetro $u \in \mathbb{R}^{\mathrm{q}}$. Entäo todos os germes $F_{u}$ são finitamente $\mathcal{G}$-determinados.

Demonstração: Um germe $f: \mathbb{R}^{\mathrm{n}}, 0 \rightarrow \mathbb{R}, 0$ é $\mathcal{G}$-finitamente determinado se existe $k$ tal que $\mathcal{M}_{n}^{k} \mathcal{E}_{n} \subset T \mathcal{G} \cdot f+\mathcal{M}_{n}^{r}$, com $r>k$, dado pelo teorema da determinação finita. O que é equivalente a existência de um sistema determinado formado por vetores em $T \mathcal{G} \cdot f+\mathcal{M}_{n}^{r}$ e tendo por variáveis os monômios $g$ de grau $s$, tal que $k \leq s \leq r$. Portanto a matriz $A$, associada a este sistema tem determinante não nulo.

Seja $F$ um desdobramento versal do germe $f$. A matriz $A_{u}$ associada ao sistema formado pelos vetores em $T \mathcal{G} \cdot F_{u}+\mathcal{M}_{n}^{r}$, onde as variáveis são os monômios de grau $s, \operatorname{com} k \leq s \leq r$, está suficientemente próxima da matriz $A$. Além disso, desde que a função determinante é contínua, $\operatorname{det}\left(A_{u}\right)$ é não nulo, afinal $\operatorname{det} A$ é não nulo. Portanto $F_{u}$ é $\mathcal{G}$-finitamente determinado. 
A partir da proposição anterior, temos que se um elemento $f_{u}$ de uma deformação de $f$ não é $\mathcal{A}^{*}$-finitamente determinado, então $f$ não é $\mathcal{A}^{*}$-finitamente determinado. Esse argumento será usado para demonstrar que a classificação obtida é completa.

Proposição 1.3.7 Seja $f: \mathbb{R}^{2}, 0 \rightarrow \mathbb{R}, 0$ germe na origem tal que $j^{1} f(0,0) \neq 0$. Se $f$ têm contato $\geq 4$ na origem com $y=0$, então o germe não é finitamente $\mathcal{A}^{*}$-determinado.

Demonstração: Pelo Lema Preparatório, se um germe tem contato $k \geq 4$ na origem ele pode ser escrito da forma

$$
f(x, y)=y+x h_{1}(y)+x^{2} h_{2}(y)+x^{k-2} h_{k-2}(y)+x^{k}
$$

com $k \geq 4$.

Além disso existe uma deformação do germe $f$ tal que $f_{u}(x, y, u)=y+\left(u_{1} y+h_{1}(y)\right) x+$ $\left(u_{2} y+h_{2}(y)\right) x^{2}+\ldots+\left(u_{4}+h_{4}(y)\right) x^{4}+\ldots+x^{k}$. Este germe por sua vez, é equivalente ao germe $\left.\overline{f_{u}}(x, y, \tilde{u})\right)=y+\left(\tilde{u}_{1} y+\tilde{h}_{1}(y)\right) x+\left(\tilde{u}_{2} y+\tilde{h}_{2}(y)\right) x^{2}+x^{4}$ se $u_{4} \neq 0$. Logo $\overline{f_{u}}$ não é germe $\mathcal{A}^{*}$-finitamente determinado pela Proposição 1.3 .5 , portanto não existe germe $\mathcal{A}^{*}$-finitamente determinado com contato $\geq 4$ pela Proposição 1.3.6.

\subsection{Classificação dos pares de 1-formas regulares}

Para o estudo dos pares de 1-formas regulares precisamos da seguinte definição.

Definịção 1.4.1 Dados dois germes de 1-formas diferenciais $\alpha$ e $\beta$ definimos o conjunto discriminante do par $(\alpha, \beta)$ como

$$
\Delta=\{(x, y):(\alpha \wedge \beta)(x, y)=0\}
$$

Além disso, dado $p \in \Delta$, diremos que p é um ponto do Tipo 2, se p pertence a uma folha comum a ambas as 1-formas, caso contrário p é dito do Tipo 1. Veja Figura 1.1.

Note que se

$$
\begin{aligned}
& \alpha=A(x, y) d x+B(x, y) d y \\
& \beta=M(x, y) d x+N(x, y) d y
\end{aligned}
$$

e $\delta=A N-B M$, então o conjunto $\triangle$ é a pré-imagem do zero pela função $\delta$. Ainda mais,

Proposição 1.4.2 Se $(\alpha, \beta) e(\gamma, \eta)$ são suavemente equivalentes então seus discriminantes são difeomorfos. 


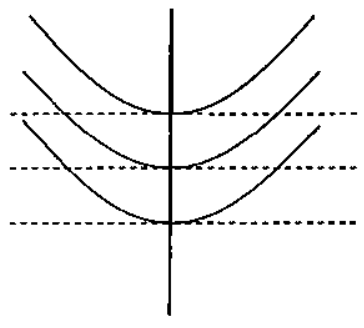

Tipo 1

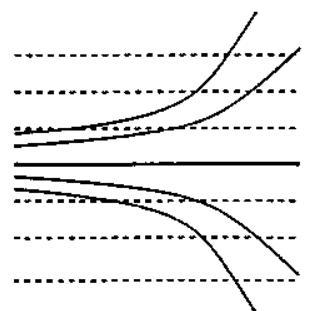

Tipo 2

Figura 1.1: Discriminante de um par'de 1-formas

Demonstração: Seja $\Phi$ uma mudança de coordenadas que leva as folhas de $(\alpha, \beta)$ às folhas de $(\gamma, \eta)$. Escrevendo $\Phi=(\phi, \psi)$, temos

$$
\begin{array}{ll}
x=\phi(X, Y) & d x=\phi_{X} d X+\phi_{Y} d Y \\
y=\psi(X, Y) & d y=\psi_{X} d X+\psi_{Y} d Y
\end{array}
$$

então $\phi_{X} \psi_{Y}-\phi_{Y} \psi_{X} \neq 0$ e enquanto que $\gamma=\left(A(\phi, \psi) \phi_{X}+B(\phi, \psi) \psi_{X}\right) d X+\left(A(\phi, \psi) \phi_{Y}+\right.$ $\left.B(\phi, \psi) \psi_{Y}\right) d Y$ e $\eta=\left(M(\phi, \psi) \phi_{X}+N(\phi, \psi) \psi_{X}\right) d X+\left(M(\phi, \psi) \phi_{Y}+N(\phi, \psi) \psi_{Y}\right) d Y$. Conseqüentemente, o discriminante do $\operatorname{par}(\gamma, \eta)$ é dado por

$$
\begin{aligned}
\delta(X, Y) & =(A N-B M)(\phi, \psi)\left(\phi_{X} \psi_{Y}-\phi_{Y} \psi_{X}\right)(X, Y) \\
& =\delta(\phi(X, Y), \psi(X, Y))\left(\phi_{X} \psi_{Y}-\phi_{Y} \psi_{X}\right)(X, Y)
\end{aligned}
$$

Ou seja, $\bar{\delta}(X, Y)=k(X, Y) \cdot \delta \circ \Phi(x, y)$, onde $k$ é uma função não nula.

Observamos que em muitas situações, abusando da linguagem, diremos que dois pares de 1-formas $(d y, d f)$ e $(d y, d g)$ são $\mathcal{A}^{*}$-equivalentes, em vez de dizer que os pares são suavemente equivalentes desde que $f \simeq \mathcal{A}^{*} g$.

Da classificação dos germes de funções pelo grupo $\mathcal{A}^{*}$ temos o seguinte teorema:

Teorema 1.4.3 Sejam $\alpha$ e $\beta$ dois germes de 1-formas diferenciais regulares com discriminante consistindo de pontos do Tipo 1. Então temos as seguintes possibilidades para o par $(\alpha, \beta)$ :

(i) Se as duas 1 -formas forem transversais, então o par $(\alpha, \beta)$ é suavemente equivalente a $(d y, d x)$, o qual é estável. Ver Figura 1.2(i).

(ii) Se as duas 1-formas têm contato 2 na origem então o discriminante é uma curva suave $e$ o par $(\alpha, \beta)$ é suavemente equivalente ao par $\left(d y, d\left(y-x^{2}\right)\right)$, o qual é estável. Ver Figura 1.2(ii).

(iii) Suponha que as 1-formas têm contato 3 na origem. Então o discriminante tem uma 
singularidade $A_{k \geq 0}$. Se $k \leq \infty,(\alpha, \beta)$ é suavemente equivalente a um dos pares abaixo:

$$
\begin{array}{ll}
3_{2 p+1}: & \left(d y, d\left(y+x y^{2 p+1}+x^{3}\right)\right), p \geq 0 \\
3_{2}: & \left(d y, d\left(y \pm x y^{2}+x^{3}\right)\right) \\
3_{2 p}: & \left(d y, d\left(y+x\left( \pm y^{2 p}+\lambda y^{5 p-1}\right)+x^{3}\right)\right), p \geq 2,
\end{array} .
$$

onde $\lambda$ é um módulo suave. Quando o discriminante tem uma singularidade $A_{\infty}$, existe difeomorfismo formal levando o par $(\alpha, \beta)$ ao par $\left(d y, d\left(y+x^{3}\right)\right)$.

As deformações versais destes pares são dadas por:

$$
\begin{aligned}
& \left(d y, d\left(y+x\left(y^{2 p+1}+u_{2 p-1} y^{2 p-1}+\cdots+u_{0}\right)+x^{3}\right)\right) \\
& \left(d y, d\left(y+x\left( \pm y^{2}+u_{0}\right)+x^{3}\right)\right) \\
& \left(d y, d\left(y+x\left( \pm y^{2 p}+\left(\lambda+u_{2 p-1}\right) y^{5 p-1}+u_{2 p-2} y^{2 p-2}+\cdots+u_{0}\right)+x^{3}\right)\right)
\end{aligned}
$$

respectivamente. Em particular, $3_{1}$ é o caso estável.

(iv) Se o contato é $\geq 4$, näo existem modelos suaves de codimensão finita para o par de 1formas.

Demonstração: Dado o par $(\alpha, \beta)$ de 1-formas regulares, podemos supor que $\alpha=d y$ (Teorema da Retificação, [2]) e $\beta=d f$. Então o discriminante do par de 1-formas é dado por $f_{x}(x, y)=0$. Desde que $y=0$ é folha de $\alpha$ e por hipótese o discriminante possui apenas pontos do Tipo 1 , temos $f^{-1}(0)$ não coincide com a curva $y=0$.

(i), (ii) Supor que as 1-formas são transversais ou têm contato 2 na origem significa que $f$ é $\mathcal{A}^{*}$-equivalente a $f(x, y)=x+y$ ou $f(x, y)=y-x^{2}$, respectivamente (ver Proposição 1.3.3), portanto $(d y, d f)$ é suavemente equivalente a $(d y, d x)$ ou $\left(d y, d\left(y-x^{2}\right)\right)$, respectivamente. Além disso estes pares são estáveis desde que $x$ e $y-x^{2}$ são germes $\mathcal{A}^{*}$-estáveis e o grupo $\mathcal{A}^{*}$ preserva as folhas da 1-forma $d y$.
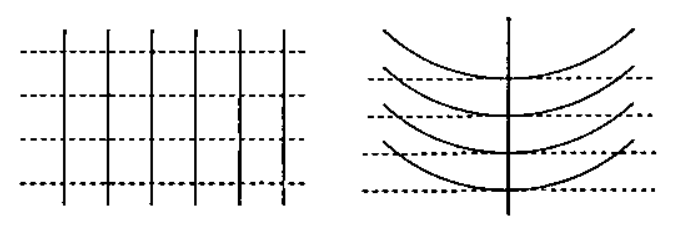

Figura 1.2: Pares regulares estáveis:(i) $(d y, d x)$ à esquerda, (ii) $\left(d y, d\left(y-x^{2}\right)\right)$ à direita

(iii) Se o contato entre as folhas de $\alpha$ e $\beta$ na origem é 3 vimos na Proposição 1.3.4 que $f$ é equivalente a um dos pares de 1-formas acima e como o desdobramento versal depende apenas do germe $f$ temos as deformações apresentadas anteriormente.

(iv) Se o contato é $\geq 4$ as Proposições 1.3.5 e 1.3.6 asseguram a não existência de modelos $\mathcal{A}^{*}$-finitamente determinados para a função $f$, portanto não temos pares de 1 -formas regulares no plano de codimensão finita com contato $\geq 4$. 
Observações 1.4.4 1. Na Figura 1.2 assim como nas figuras que seguem, as retas tracejadas indicam as folhas da 1-forma dy, a curva em negrito é o discriminante e as demais curvas são as folhas da 1-forma $d f$.

2. No Teorema 1.4.3 temos a lista completa dos pares de 1-formas diferenciais no plano de codimensão finita, a menos de mudanças suaves, desde que a $\mathcal{A}^{*}$-classificação dos germes $\mathbb{R}^{2}, 0 \rightarrow \mathbb{R}, 0$ obtida no Teorema 1.3 .1 é completa.

3. Mancini, Ruas e Teixeira, [23] obtiveram a classificação formal completa dos diagramas divergentes do tipo $(f, g):(\mathbb{R}, 0) \leftarrow\left(\mathbb{R}^{2}, 0\right) \rightarrow(\mathbb{R}, 0)$ de codimensão finita. Eles reduziram o problema à classificação de germes de funções $\mathbb{R}^{2}, 0 \rightarrow \mathbb{R}, 0$. Notamos que as formas normais por eles obtidas são as mesmas que encontramos na $\mathcal{A}^{*}$-classificação. Porém na classificação dos diagramas divergentes o grupo de mudanças de coordenadas não é geométrico como ocorre com o grupo $\mathcal{A}^{*}$. Sendo assim a classificação dos diagramas divergentes é apenas formal, enquanto que a classificação dos pares de 1-formas é suave.

\subsection{Estudo geométrico}

Dado o par de germes de 1-formas diferenciais $(d y, d f)$, temos $\delta(x, y)=f_{x}(x, y)$. Além disso, se $f$ é um germe $\mathcal{A}^{*}$-finitamente determinado, todas as informaçōes sobre o par $(d y, d f)$ são dadas pelas $\mathcal{A}^{*}$-singularidades de $f$. Em particular todas as deformações do par podem ser obtidas através das deformações de $f$. Desde que as deformações de $f$ são obtidas a partir de um desdobramento versal, basta estudarmos a geometria do desdobramento versal de $f$.

Notamos que os únicos germes de interesse são aqueles com singularidade $3_{k}, k \geq 1$. Neste caso o conjunto de bifurcação de um desdobramento versal $F$ é o conjunto dos valores de $u$ para os quais o contato entre $d y$ e $d f^{u}$ na origem é 3 e o discriminante do par de folheações é singular.

Logo podemos escrever

$$
\begin{aligned}
& B i f(F)=\left\{u: \exists x \text { com } f^{u} \mathcal{A}^{*}-\text { instável em } x\right\} \\
& B i f(F)=\left\{u: f_{x}^{u}=f_{x x}^{u}=f_{x y}^{u}=0\right\}
\end{aligned}
$$

- Dado o par $\left(d y, d\left(y+x y+x^{3}\right)\right)$, temos $\delta(x, y)=3 x^{2}+y$, isto é, o discriminante é uma parábola (uma curva suave), logo o conjunto de bifurcação é vazio. Veja Figura 1.3.

- Dada a família $\left(d y, d\left(y+x\left( \pm y^{2}+u_{0}\right)+x^{3}\right)\right.$, temos $\delta^{u}(x, y)= \pm y^{2}+3 x^{2}+u_{0}$, ou seja, o discriminante $\delta^{0}$ tem uma singularidade Morse e a família $\delta^{u}$ é um desdobramento versal desta singularidade, sendo assim $\delta^{0}$ passa pelas deformaçōes genéricas de Morse (ver Figura 1.4). O conjunto de bifurcação neste caso é um ponto isolado, a origem, que 


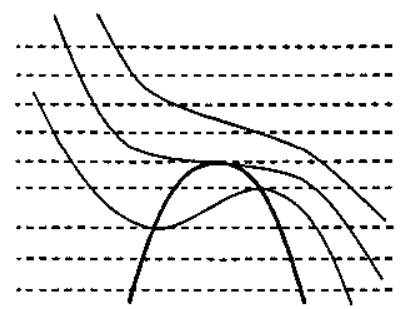

Figura 1.3: As folheações do par $\left(d y, d\left(y+x y+x^{3}\right)\right)$

é o $\mathcal{R}$-conjunto de bifurcação da singularidade $A_{1}$ do discriminante. Na Figura 1.4 temos acima as deformações genéricas de uma singularidades $A_{1}^{+}$do discriminante e, abaixo, $A_{1}^{-}$.

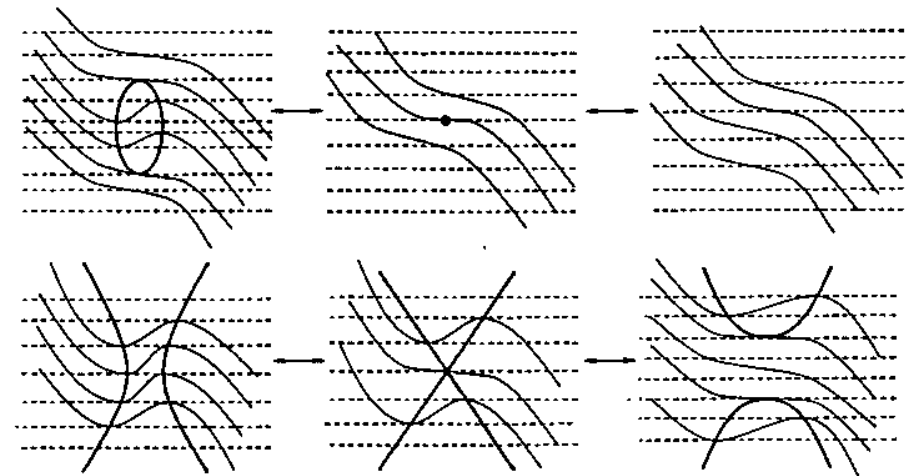

Figura 1.4: Deformaçōes genéricas dos pares de 1-formas: $\left(d y, d\left(y+x y^{2}+x^{3}\right)\right.$ (acima) e $\left(d y, d\left(y-x y^{2}+\right.\right.$ $\left.\left.x^{3}\right)\right)$ (abaixo)

- Dada a família $\left(d y, d\left(y+x\left(y^{3}+u_{0} y+u_{1}\right)+x^{3}\right)\right)$ e denotando por $u=\left(u_{0}, u_{1}\right)$, temos $\delta^{u}(x, y)=y^{3}+3 x^{2}+u_{0} y+u_{1}$. Note que para $u=(0,0)$ o discriminante apresenta singularidade $A_{2}$ (cúspide) com $\delta^{u}$ um desdobramento versal de tal singularidade. Ao conjunto de bifurcação pertencem os pontos $u=\left(u_{0}, u_{1}\right)$ tais que $\delta^{u}$ tem singularidade $A_{\geq 1}$. Estes pontos satisfazem as equaçōes

$$
\left\{\begin{array}{l}
\delta=3 x^{2}+y^{3}+u_{0} y+u_{1}=0 \\
\delta_{x}=6 x=0 \\
\delta_{y}=3 y^{2}+u_{0}=0
\end{array}\right.
$$

ou seja, os pontos da forma $\left(u_{0}, u_{1}\right)=\left(-3 y^{2}, 2 y^{3}\right)$ (cúspide). O diagrama de bifurcação da família é dado na Figura 1.5, onde a cada ponto do conjunto dos parâmetros $\left(u_{0}, u_{1}\right)$ temos o par de germes para esses valores dos parâmetros.

Observemos ainda que o conjunto de bifurcação divide o conjunto dos parâmetros em dois abertos, em ambos o discriminante é regular. Em um dos ramos da cúspide o discriminante tem uma singularidade $A_{1}^{+}$e no outro ramo, $A_{1}^{-}$. O par de folheaçōes realizam as bifurcações da Figura 1.4 atravessando os ramos da cúspide. 


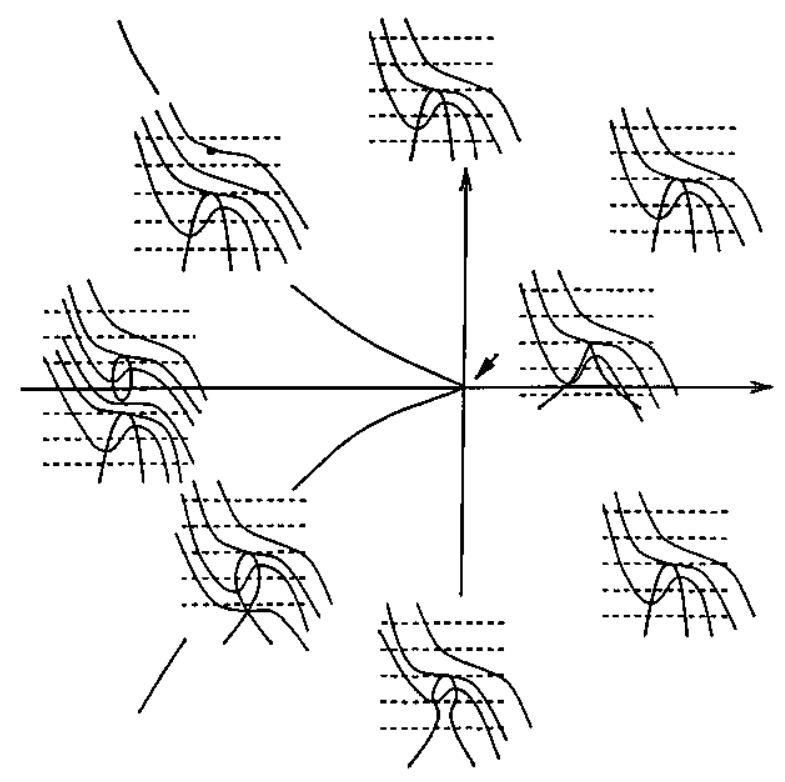

Figura 1.5: Diagrama de bifurcação do par de germes $\left(d y, d\left(y+x y^{3}+x^{3}\right)\right)$

Neste estudo geométrico, assim como nos demais capítulos, estudamos com mais detalhes os germes de codimensão menor ou igual a 2 , desenhamos seus desdobramentos versais e observamos o que ocorre nos conjuntos de bifurcação.

Observação 1.5.1 (1) Em geral, dada a familia $\left(d y, d\left(y+x\left( \pm y^{2 p+1}+u_{2 p-1} y^{2 p-1}+\ldots+u_{0}\right)+\right.\right.$ $\left.x^{3}\right)$, temos $\delta^{u}(x, y)=3 x^{2} \pm y^{2 p+1}+u_{2 p-1} y^{2 p-1}+\ldots+u_{0}$, ou seja, o discriminante $\delta^{0}$ tem singularidade $A_{2 p}$ e a familia $\delta^{u}$ é um $\mathcal{R}$-desdobramento versal desta singularidade. $O$ mesmo acontece com a familia dada por $\left(d y, d\left(y+x\left( \pm y^{2 p}+\left(\lambda+u_{2 p-1}\right) y^{5 p-1}\right)+u_{2 p-2} y^{2 p-2}+\right.\right.$ $\left.\left.\left.\ldots+u_{0}\right)+x^{3}\right)\right)$. $O$ discriminante $\delta^{0}$ tem singularidade $A_{2 p-1}$ e $\delta^{u}$ é um $\mathcal{R}$-desdobramento versal de tal singularidade. O conjunto de bifurcação da deformação de $f$ é igual ao $\mathcal{R}$ conjunto de bifurcaçāo da singularidade $A_{k}$ do discriminante $(k=2 p$, ou $k=2 p-1)$.

(2) Na Proposição 1.3.4 encontramos um germe com módulo, isto é, um escalar $\lambda$ que não pode ser eliminado por mudanças em $\mathcal{A}^{*}$. No entanto observemos que tal módulo nāo altera a configuração do par de 1-formas, o tipo de singularidade do discriminante nem suas deformações (se $\lambda \neq 0$ ). Na seção 1.8 tratamos da eliminação desse módulo.

\subsection{Invariantes}

Dado um germe $f: \mathbb{R}^{2}, 0 \rightarrow \mathbb{R}, 0$ finitamente determinado temos invariantes a ele associados, por exemplo, o grau da $\mathcal{A}^{*}$-determinação de $f$ e a $\mathcal{A}^{*}$-codimensão deste germe são invariantes naturais vindos da teoria de singularidades. 
Dado um par de germes de 1-formas $(d y, d f)$ no plano, onde $f$ é germe $\mathcal{A}^{*}$-finitamente determinado, vimos que uma deformação versal deste par é dada por $(d y, d F)$, onde $F$ é uma deformação versal de $f$.

Além disso observamos que existe uma singularidade estável que aparece em número finito nas deformações dos pares instáveis. Esta singularidade é caracterizada pelo contato de ordem 3 da folha de $\beta$ com a folha de $\alpha$ enquanto que o discriminante tem contato 2 com essa mesma folha. Usando a convenção anterior $(\alpha, \beta)=(d y, d f)$, esta singularidade é caracterizada por $f_{x}=f_{x x}=0, f_{x x x} \neq 0, f_{x y} \neq 0$ e é modelada pela singularidade $3_{1}$ do Teorema 1.4.3. Dado um germe $f: \mathbb{R}^{2}, 0 \rightarrow \mathbb{R}, 0$ definimos o seguinte número

$$
\sharp 3_{1}(f)=\operatorname{dim}_{\mathbb{R}} \frac{\mathcal{E}_{2}}{\left.<f_{x}, f_{x x}\right\rangle} .
$$

Lembremos que a multiplicidade de um germe de aplicação holomorfa $F: \mathbb{C}^{n}, 0 \rightarrow \boldsymbol{C}^{p}, 0$ dado por $F=\left(f_{1}, \ldots, f_{p}\right)$, é definido como sendo a dimensão do ideal gerado por $\left\{f_{1}, \ldots, f_{p}\right\}$, ou seja,

$$
\operatorname{dim}_{\mathbf{C}_{<f_{1}, \ldots, f_{p}>}} \frac{\dot{\mathcal{O}}_{n}}{}
$$

que representa o número de soluções do sistema $f_{1}=\ldots=f_{p}=0$, quando $F$ é deformada. Para um germe real nem sempre encontramos o mesmo número de soluções para esse sistema, no entanto podemos interpretar a dimensão acima como sendo o número máximo de soluções que o sistema pode ter. Em alguns casos podemos garantir que o número máximo de soluções é atingido por uma deformação real de $F$.

Abusando da notação, denotamos-o por $\sharp 3_{1}(f)$ em vez de $\sharp 3_{1}(d y, d f)$ e assim podemos interpretar $\sharp 3_{1}(f)$ como sendo o número máximo de pontos caracterizados por $f_{x}=f_{x x}=0$ que aparecem numa deformação versal do par $(d y, d f)$.

Mostramos no lema abaixo que $\sharp 3_{1}$ como definido acima é um invariante suave para pares de germes de 1-formas regulares no plano.

Lema 1.6.1 Dados dois germes $f, g: \mathbb{R}^{2}, 0 \rightarrow \mathbb{R}, 0$ tal que $f \simeq \mathcal{A}^{*} g$ temos

$$
\sharp 3_{1}(d y, d f)=\sharp 3_{1}(d y, d g) .
$$

Demonstração: Desde que $f \simeq_{\mathcal{A}^{*}} g$ existem $(h, k)=\left(\left(h^{1}(x, y), h^{2}(y)\right), k(z)\right) \in \mathcal{A}^{*}$ tal que $g=k \circ f \circ h$. Vamos mostrar que

$$
\operatorname{dim}_{\mathbb{R}} \frac{\mathcal{E}_{2}}{<g_{x}, g_{x x}>}=\operatorname{dim}_{\mathbb{R}} \frac{\mathcal{E}_{2}}{\left.<f_{x}, f_{x x}\right\rangle} .
$$

Observe que

$$
\begin{aligned}
& g_{x}=k^{\prime}(f \circ h) \cdot\left(f_{x} \circ h\right) \cdot h_{x}^{1} \\
& g_{x x}=k^{\prime \prime}(f \circ h) \cdot\left[\left(f_{x} \circ h\right) \cdot h_{x}^{1}\right]^{2}+k^{\prime}(f \circ h) \cdot\left[\left(f_{x x} \circ h\right) \cdot\left(h_{x}^{1}\right)^{2}+\left(f_{x} \circ h\right) \cdot h_{x x}^{1}\right] .
\end{aligned}
$$


Supondo que $\frac{\mathcal{E}_{2}}{\left\langle f_{x}, f_{x x}\right\rangle}=\mathbb{R} \cdot\left\{\theta_{1}, \ldots \theta_{p}\right\}$, para todo $H \in \mathcal{E}_{2}$ existem únicos $\lambda_{i} \in \mathbb{R}, i=1, \ldots, p$ e $\phi_{1}, \phi_{2} \in \mathcal{E}_{2}$ tal que $H=\sum_{i=1}^{p} \lambda_{i} \theta_{i}+\left(\phi_{1} f_{x}+\phi_{2} f_{x x}\right)$.

Dados $e_{1}, e_{2}, e_{3}$ funçōes unitárias $\left(e_{i}(0) \neq 0\right)$ temos

$$
\frac{\mathcal{E}_{2}}{<e_{1} f_{x}, e_{2} f_{x}+e_{3} f_{x x}>}=\mathbb{R} \cdot\left\{\theta_{1}, \ldots \theta_{p}\right\}
$$

Além disso

$$
\frac{\mathcal{E}_{2}}{<f_{x} \circ h, f_{x x} \circ h>}=\mathbb{R}\left\{\theta_{1} \circ h, \ldots \theta_{p} \circ h\right\} .
$$

Portanto a codimensão dos ideais $<f_{x}, f_{x x}>\mathrm{e}<g_{x}, g_{x x}>$ são coincidentes se $f$ e $g$ são germes $\mathcal{A}^{*}$-equivalentes.

No entanto $\sharp 3_{1}$ não é um invariante topológico, como veremos através do seguinte exemplo:

Exemplo 1.1 Considere os pares $\left(d y, d\left(y+x^{2}\right)\right) e\left(d y, d\left(y+x^{2} y^{2}+x^{4}\right)\right)$, denotados por $(d y, d f)$ $e(d y, d g)$, respectivamente.

Primeiramente notamos que existe um homeomorfimo $h$ preservando as folhas da 1 -forma $d y$, dado por $h(x, y)=\left(x\left(x^{2}+y^{2}\right)^{1 / 2}, y\right)$ tal que $g \circ h=f$, ou seja $f$ e $g$ são topologicamente equivalentes.

Mas

$$
\sharp 3_{1}(f)=\operatorname{dim}_{\mathbb{R}} \frac{\mathcal{E}_{2}}{<2 x, 2>}=0
$$

enquanto que

$$
\sharp 3_{1}(g)=\operatorname{dim}_{\mathbb{R}} \frac{\cdot \mathcal{E}_{2}}{<4 x^{3}+2 x y^{2}, 12 x^{2}+2 y^{2}>}=\operatorname{dim} \mathbb{R} .\left\{1, x, x^{2}, y, x y, x^{2} y\right\}=6 .
$$

Portanto $\sharp 3_{1}$ não é um invariante topológico.

Observação 1.6.2 O cálculo da codimensão do ideal acima foi feita com o auxílio do software Maple. Seguindo os comandos

$>$ with(grobner):

$>I:=\left[f_{x}, f_{x x}\right]:$

$>\operatorname{gbasis}(I, \operatorname{tdeg}(x, y))$;

temos a base $J$ de Grobner do ideal I de acordo com a ordem lexicográfica definida $(x>y)$. Calcule então os termos líderes desse ideal $(L T(J))$ e construa o ideal $K=\langle L T(J)\rangle$. Os elementos divisiveis por $L T(J)$ formam um base para $\frac{\mathcal{E}_{2}}{\langle I\rangle}$.

No caso da família $\left(d y, d\left(y \pm x y^{k}+x^{3}+u_{k-2} x y^{k-2}+\ldots+u_{0} x\right)\right), \sharp 3_{1}$ depende do número máximo de zeros da equação $3 \pm y^{k}+u_{k-2} y^{k-2}+\ldots+u_{0}=0$. Note que se escolhemos convenientemente os valores de $u_{k-2}, \ldots, u_{0}$ temos $k$ soluções para a equação acima, portanto $\sharp 3_{1}(f)=k$. Note 
ainda que no caso de $k=2$, o desdobramento versal do par $\left(d y, d\left(y \pm x y^{2}+x^{3}\right)\right.$ ) (ver Figura 1.4) tem em um dos lados da transição 2 pontos de tipo $3_{1}$ e nenhum ponto do outro. $O$ que mostra que o número máximo de pontos $3_{1}$ neste caso é atingido por uma deformação versal deste par. Olhando para o caso $k=3$, temos que o conjunto de bifurcação define dois abertos numa vizinhança da origem onde os pares são estáveis (ver Figura 1.5), então observamos 3 pontos do tipo $3_{1} \mathrm{em}$ um aberto enquanto que apenas 1 no outro, sendo que sobre o conjunto de bifurcação temos sempre 2 pontos do tipo $3_{1}$, portanto $\sharp 3_{1}$ também é atingido neste caso. Uma pergunta natural é se isso ocorre em geral. A resposta é dada pela proposição abaixo.

Proposição 1.6.3 Dados os pares de germes $(d y, d f)=\left(d y, d\left(y+x y^{2 p+1}+x^{3}\right)\right) e(d y, d g)=$ $\left(d y, d\left(y+x\left( \pm y^{2 p}+\lambda y^{5 p-1}\right)+x^{3}\right)\right)$ temos $\sharp 3_{1}(f)=2 p+1 e \sharp 3_{1}(g)=2 p$. Além disso, o número máximo de pontos $3_{1}$ é observado numa deformação real dos pares de germes acima.

Demonstração: Uma deformação versal do primeiro par acima é dada pela família $(d y, d(y+$ $\left.\left.x y^{2 p+1}+x^{3}+u_{k-2} x y^{k-2}+\ldots+u_{0} x\right)\right)$ com $k \geq 2$ logo temos

$$
\begin{aligned}
f_{x}^{u} & =3 x^{2} \pm y^{2 p+1}+u_{2 p} y^{2 p-1}+\ldots+u_{1} y+u_{0} \\
f_{x x}^{u} & =x
\end{aligned}
$$

e o número de zeros do polinômio $\pm y^{2 p+1}+u_{2 p} y^{2 p-1}+\ldots+u_{1} y+u_{0}$ é que determinam o número de pontos $3_{1}$ que aparecem num desdobramento deste par. Desde que existem $u_{2 p}, \ldots, u_{1}, u_{0}$ de modo que o polinômio acima admite $2 p+1$ raízes, o número máximo de pontos $3_{1}$ que é $2 p+1$ é atingido.

Analogamente para o segundo par, uma deformação versal é dada pela família $(d y, d(y \pm$ $\left.\left.x y^{2 p}+\lambda x y^{5 p-1}+x^{3}+u_{2 p-2} x y^{2 p-2}+\ldots+u_{0} x\right)\right)$ com $k \geq 0$. Entāo procedendo como acima teremos $2 p$ raízes.

\subsection{Eliminação do módulo na classificação suave}

Observamos que o módulo $\lambda$ não altera a geometria do par de germes de 1-formas no plano dado por $\left(d y, d\left(y+x\left( \pm y^{2 p}+\lambda y^{5 p-1}\right)+x^{3}\right)\right)$, o discriminante tem singularidade $A_{2 p-1}, \sharp 3_{1}=2 p-1$, para todo $\lambda \in \mathbb{R}$. Parece bastante natural questionarmos a possibilidade de eliminarmos esse módulo por uma relação de equivalência via homeomorfismos.

Lembremos que duas folheaçōes $(\alpha, \beta)$ e $(\gamma, \eta)$ são topologicamente equivalentes se existe um homeomorfismo que leva as folhas da 1-forma $\alpha$ nas folhas da 1-forma $\gamma$ e leva as folhas da 1-forma $\beta$ nas folhas da 1-forma $\eta$.

No próximo teorema demonstramos que os pares $3_{2}^{+}$são topológicamente equivalentes ao par $(d y, d x)$. 
Teorema 1.7.1 O par de 1-formas regulares e suaves $\left(d y, d\left(y+x\left(y^{2 p}+\lambda y^{5 p-1}\right)+x^{3}\right)\right)$ é topologicamente equivalente ao par $(d y, d x)$.

Demonstração: Vamos construir uma vizinhança $U$ da origem e verificar que existe um homeomorfismo levando as folhas do par de germe $\left(d y, d\left(y+x\left(y^{2 p}+\lambda y^{5 p-1}\right)+x^{3}\right)\right)$ em $U$ nas folhas do par $(d y, d x)$.

Escolha $\varepsilon>0$ (suficientemente pequeno) e considere as curvas de nível do germe $f=$ $y+x\left(y^{2 p}+\lambda y^{5 p-1}\right)+x^{3}$ passando por $(\varepsilon, 0)$ e $(-\varepsilon, 0)$. Escolha também $\delta>0$ e considere as folhas de $d y$ por $(0, \delta)$ e $(0,-\delta)$. Por $U$ entendemos a região limitada pelas quatro curvas acima.

Denotamos por $J_{1}$ o intervalo dos pontos da forma $(x, \delta)$ da reta $y=\delta$ que estão limitados pelas intersecções das curvas de nível de $f$ por $(\varepsilon, 0)$ e $(-\varepsilon, 0)$, que denotamos por $L_{\beta}(\varepsilon)$ e $L_{\beta}(-\varepsilon)$, respectivamente. E por $I_{1}$, o intervalo dos pontos em $L_{f}(-\varepsilon)$, limitados pelas intersecções desta curva com $y=-\delta$ e $y=\delta$.

Dado $p \in U$, existem únicas folhas de $d y$ e $d f$ por $p$ (desde que o discriminante deste par é um ponto isolado), denotando-as por $L_{\beta}(p)$ e $L_{\alpha}(p)$. Seguindo-as até alcançar $J_{1}$ e $I_{1}$, respectivamente, defina os pontos intersecção $p_{1}=L_{\alpha}(p) \cap I_{1}$ e $p_{2}=L_{\beta}(p) \cap J_{1}$. Abusando da notação, chamamos $\left(p_{1}, p_{2}\right) \in I_{1} \times J_{1}$ as coordenadas de $p \in U$.

Seja $V$ uma vizinhança da origem da forma $I_{2} \times J_{2}$, onde $I_{2}=(-1,1)$ e $J_{2}=(-1,1)$. Vamos construir um homemorfismo $H: U \rightarrow V$ que leva as folhas de $(d y, d f)$ as follhas de $(d y, d x)$.

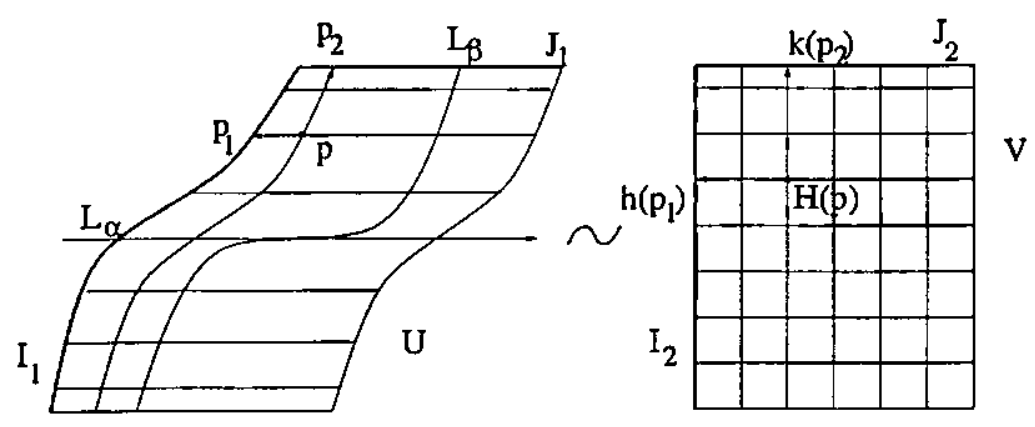

Figura 1.6: Pares topologicamente equivalentes

Considere dois homeomorfismos $h: I_{1} \rightarrow I_{2}$ e $k: J_{1} \rightarrow J_{2}$ e defina a aplicação $H: I_{1} \times J_{1} \rightarrow I_{2} \times J_{2}$ por $H(p)=H\left(p_{1}, p_{2}\right)=\left(h\left(p_{1}\right), k\left(p_{2}\right)\right)$. É fácil verificar que esta aplicação é um homeomorfimo levando as folhas do par $\left(d y, d\left(y+x\left( \pm y^{2 p}+\lambda y^{5 p-1}\right)+x^{3}\right)\right)$ que estão em $U$ às folhas do par $(d y, d x)$ em $V$. Portanto esses pares são topologicamente equivalentes.

Vale observar que neste resultado foi fundamental a hipótese sobre o discriminante ser um ponto isolado.

No teorema seguinte exibimos um modelo topológico para os pares $3_{2}^{-}$. 
Teorema 1.7.2 O par de germes de 1-formas $\left(d y, d\left(y+x\left(-y^{2 p}+\lambda y^{5 p-1}\right)+x^{3}\right)\right)$ é topologicamente equivalente ao par $\left(d y, d\left(y-x y^{2}+x^{3}\right)\right)$.

Demonstração: Observe que o discriminante do primeiro par de 1-formas é formado por duas curvas com tangência de ordem $p$ na origem enquanto que o discriminante do segundo par é formado por duas retas concorrentes pela origem, sendo assim, em ambos os pares, temos 4 regiões distintas definidas pelas curvas discriminante em qualquer vizinhança da origem. Definindo uma correspondência entre as quatro regiōes como na Figura 1.7, vamos mostrar que os dois pares de folheações são homeomorfos em cada uma dessas regiões. Mostramos também que para estes homeomorfismos a colagem fica bem definida.
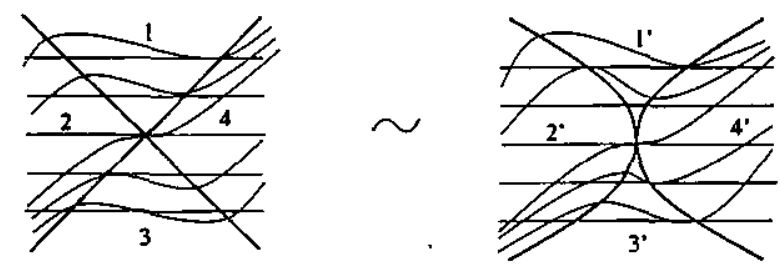

Figura 1.7: Regiões definidas pelo discriminante

Denotamos por $\alpha=d y, \beta=d\left(y-x y^{2}+x^{3}\right)$ e $\beta^{\prime}=d\left(y+x\left(-y^{2 p}+\lambda y^{5 p-1}\right)+x^{3}\right)$. Sendo que $L_{\alpha}(p)$ e $L_{\beta}(p)$ indicam as folhas de $\alpha$ e $\beta$, respectivamente, por $p$.

Região 1. Veja a Figura 1.8. Nesta região $I_{1}$ denota um segmento da semi-reta $y=-x$, para $x \leq 0$ e $J_{1}$ denota um segmento da semi-reta $y=x$, para $x \geq 0$. As folhas de $\beta$ definem um homeomorfismo $h: I_{1} \rightarrow J_{1}$, que associa a cada ponto $p \in I_{1}$, o ponto $h(p) \in J_{1}$ dado pela interseç̧ão de $J_{1}$ e $L_{\beta}(p)$. Podemos definir também um homeomorfismo $k$ em $I_{1}$ que associa a cada ponto $p$ de $I_{1} \circ$ ponto $k(p)$, intersecção de $L_{\alpha}(h(p))$ e $I_{1}$.

(1)

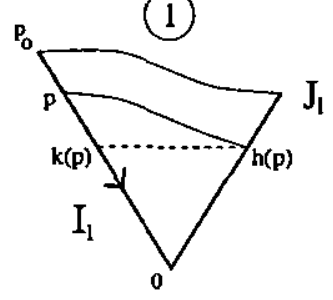

(1)

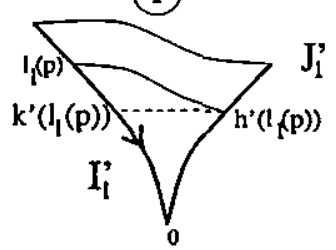

Figura 1.8: Regiāo 1 e $1^{\prime}$

Da mesma maneira definimos os homeomorfismos $h^{\prime}: I_{1}^{\prime} \rightarrow J_{1}^{\prime}$ e $k^{\prime}: I_{1}^{\prime} \rightarrow I_{1}^{\prime}$ na região $1^{\prime}$ para configuração do par $\left(\alpha, \beta^{\prime}\right)$.

Escolha a orientação para o segmento $I_{1}$ de $p_{0}$ para a origem e para $I_{1}^{\prime}$ da mesma forma, com essas orientaçōes os homeomorfismos $h, h^{\prime}, k, k^{\prime}$ são crescentes.

Observe que existe um homeomorfismo $H$ entre as regiões 1 e $1^{\prime}$ acima se, e somente se, existir um homeomorfismo crescente $l_{1}$ tal que $k^{\prime}=l_{1} \circ k \circ l_{1}^{-1}$. 
De fato, se existe tal homeomorfismo $H$, ele deve preservar as folheações e induzir um homeomorfismo $l_{1}: I_{1} \rightarrow I_{1}^{\prime}$, tal que, dado $p \in I_{1}, H\left(L_{\beta}(p)\right)=L_{\beta^{\prime}}\left(l_{1}(p)\right)$ e $H\left(L_{\alpha}(h(p))\right)=$ $L_{\alpha}\left(h^{\prime}\left(l_{1}(p)\right)\right)$. Portanto $l_{1}(k(p))=k^{\prime}\left(l_{1}(p)\right)$, ou ainda,

$$
l_{1} \circ k=k^{\prime} \circ l_{1} \text {. }
$$

Além disso, não é difícil verificar que a aplicação $l_{1}$ é crescente pela construção acima.

Inversamente, se existe um homeomorfismo crescente $l_{1}: I_{1} \rightarrow I_{1}^{\prime}$ satisfazendo (1.13) podemos construir um homeomorfismo $H$ entre as regiões 1 e $1^{\prime}$ da seguinte maneira. Seja $q$ um ponto na região 1, então $q$ é a interseç̧ão das folhas $L_{\beta}\left(q_{\beta}\right)$ e $L_{\alpha}\left(q_{\alpha}\right)$. Além disso, $q_{\beta} \leq q_{\alpha} \leq k\left(q_{\beta}\right)$, pois as folhas de $\beta$ são transversais as folhas de $\alpha$ nesta região. Desde que $l_{1}$ é crescente temos $l_{1}\left(q_{\beta}\right) \leq l_{1}\left(q_{\alpha}\right) \leq l_{1}\left(k\left(q_{\beta}\right)\right)=k^{\prime}\left(l_{1}\left(q_{\beta}\right)\right)$ (ver Figura 1.9 ).
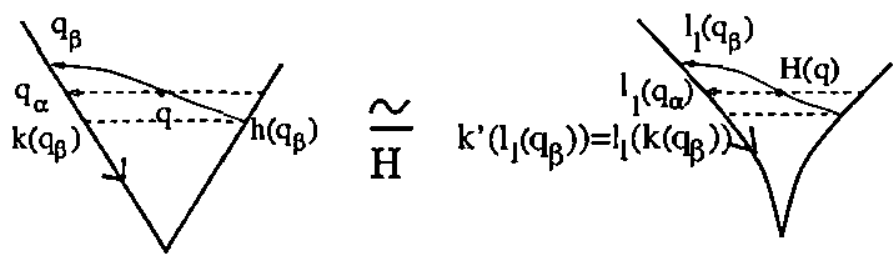

Figura 1.9: Construção do homeomorfismo na região 1

Sendo assim $L_{\beta^{\prime}}\left(l_{1}\left(q_{\beta}\right)\right)$ intercepta $L_{\alpha}\left(l_{1}\left(q_{\alpha}\right)\right)$ em um único ponto que denotamos por $H(q)$ e a aplicação $H$ assim construída é um homeomorfismo, desde que a aplicação $l_{1}$ é bijetiva e andar ao longo das folhas de $\alpha$ e $\beta$ define uma aplicação contínua com inversa contínua.

Note que o homeomorfismo $l_{1}$ existe desde que as aplicações $k, k^{\prime}$ não têm pontos fixos em $I_{1}$ e $I_{1}^{\prime}$ respectivamente, como mostrado em [35], página 19-20, o que acontece neste caso, portanto podemos garantir a existência do homeomorfismo $H$ entre as regiões 1 e $1^{\prime}$. Observe ainda que as folheações na região 1 são levadas nas folheações da região $1^{\prime}$, por essa construção .

Região 3. É tratada da mesma maneira que a região 1 acima, apenas definimos a orientação oposta para $I_{2}$ e $I_{2}^{\prime}$, isto é da origem para os extremos dos intervalos $I_{2}$ e $I_{2}^{\prime}$.

Região 2. Nesta região é preciso definir o homeomorfismo de maneira conveniente para que a colagem esteja bem definida, isto é, os homeomorfismo coincidam no conjunto discriminante e assim tenhamos um par de folheação sendo levado em outro par de folheação

Das regiões 1 e 3 , temos os homeomorfismos $l_{1}: I_{1} \rightarrow I_{1}^{\prime}$ e $l_{2}: I_{2} \rightarrow I_{2}^{\prime}$. Defina a aplicação $l: I_{1} \cup I_{2} \rightarrow I_{1}^{\prime} \cup I_{2}^{\prime}$ satisfazendo as seguintes condições $l_{I_{1}}=l_{1}$ e $l_{I_{2}}=l_{2}$. Então dado $q$ na região 2, existirão $q_{\alpha}$ e $q_{\beta}$ em $I_{1} \cup I_{2}$ definidos pela interseç̧ão das folhas $L_{\beta}\left(q_{\beta}\right)$ e $L_{\alpha}\left(q_{\alpha}\right)$ com $I_{1} \cup I_{2}$, sendo que $q_{\alpha} \geq q_{\beta}$ (orientação definida nas regiōes 1 e 3 ), portanto, $L_{\beta^{\prime}}\left(l\left(q_{\beta}\right)\right.$ ) e $L_{\alpha}\left(l\left(q_{\alpha}\right)\right)$ se interceptam em um ponto da região $2^{\prime}$, que denotaremos por $H(q)$.

A aplicação $H$ está bem definida, afinal $\left.H\right|_{I_{1}}=l_{1}$ e $\left.H\right|_{I_{2}}=l_{2}$, donde temos que a colagem está bem definida também e é homeomorfismo por construção. 

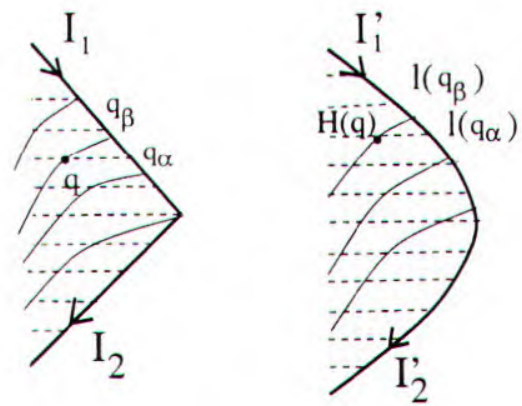

Figura 1.10: O homeomorfismo na região 2

Região 4. Como na região 2, usaremos os homeomorfismos $l_{1}$ e $l_{2}$ definidos anteriormente para definir homeomorfismos $g_{1}: J_{1} \rightarrow J_{1}^{\prime}$ e $g_{2}: J_{2} \rightarrow J_{2}^{\prime}$. Consideramos que $J_{1}$ está orientado de $q_{0}$ para a origem, assim como $J_{1}^{\prime}$ enquanto que $J_{2}$ e $J_{2}^{\prime}$ tem a orientação da origem para os extremos (ver Figura 1.11).

Para todo $q \in J_{1}$, existe um único $p \in I_{1}$ tal que $q=h(p)$, ou seja, $p=h^{-1}(q)$. Do homeomorfismo definido na região 1 temos que $q=h(p)$ é levado em $h^{\prime}\left(l_{1}(p)\right)=h^{\prime}\left(l_{1}\left(h^{-1}(q)\right)\right)$. Logo definimos $g_{1}(q)=\left(h^{\prime} \circ l_{1} \circ h^{-1}\right)(q)$ em $J_{1}$. Analogamente definimos $g_{2}=h_{2}^{\prime} \circ l_{2} \circ h_{2}^{-1}$, onde $h_{2}^{\prime} \mathrm{e}$ $h_{2}$ são os homeomorfismos obtidos na construção do homeomorfismo entre as regiões 3 e $3^{\prime}$.
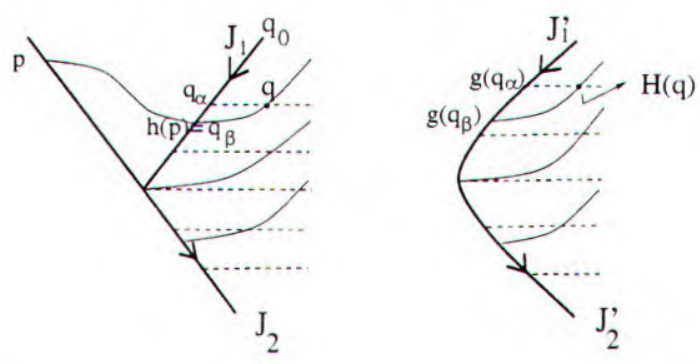

Figura 1.11: O homeomorfismo na região 3

Considere $g: J_{1} \cup J_{2} \rightarrow J_{1}^{\prime} \cup J_{2}^{\prime}$ tal que $\left.g\right|_{J_{1}}=g_{1}$ e $\left.g\right|_{J_{2}}=g_{2}$.

Além disso, dado um ponto $q$ na região 4 , existem os pontos $q_{\beta}$ e $q_{\alpha}$, intersecção de $L_{\beta}(q)$ e $L_{\alpha}(q)$ com $J_{1}$ satisfazendo $q_{\alpha} \leq q_{\beta}$, logo as folhas $L_{\beta}\left(g\left(q_{\beta}\right)\right)$ e $L_{\alpha}\left(g\left(q_{\alpha}\right)\right)$ também se interceptam na região $4^{\prime}$ pois $g$ é crescente nesta região. Definimos então a aplicação $H$, que associa ao ponto $q$ o ponto definido pela intersecção acima (ver a Figura 1.11). $H$ é um homeomorfismo e define uma boa colagem das regiões $1,2,3$ e 4 .

Observe ainda, que pela construção acima o par de folheações $(\alpha, \beta)$ é levado ao par de folheações $\left(\alpha, \beta^{\prime}\right)$ nas respectivas regiões, sendo ainda que a colagem esta bem definida, pois os homeomorfismos coincidem no bordo das regiões consideradas. Portanto podemos concluir que o pares de folheação $(\alpha, \beta)$ é levado ao par $\left(\alpha, \beta^{\prime}\right)$ pelo homeomorfismo construído nesta proposição. 


\section{Capítulo 2}

\section{Classificação suave de pares de 1-formas diferenciais $(d y, d f)$ em variedades de dimensão 2}

No capítulo anterior estudamos pares de germes de 1-formas do tipo regular/regular no plano e vimos que sempre podemos escrever o $\operatorname{par}(\alpha, \beta)$ da forma $(d y, d f)$, onde $f$ é germe de função diferenciável definida em $\mathbb{R}^{2}, 0$. Neste capítulo queremos estudar os pares de germes de 1-formas diferenciais $(\alpha, \beta)$ no plano, onde a folheação $\alpha$ é regular e $\beta$ é uma folheação singular. Neste caso não podemos assegurar que $\beta$ é 1-forma exata, então vamos restringir nosso estudo àquelas folheações onde $\beta$ é definida pelas curvas de nível de um germe de função $f: \mathbb{R}^{2}, 0 \rightarrow \mathbb{R}, 0$ singular e classificar pares de folheaçōes da forma $(d y, d f)$. Os casos estáveis e de codimensāo 1 foram estudados por Teixeira em [37] e [38]. Em [37] encontramos ainda um exemplo de um par estável sobre $S^{2}$, que em uma situação idealizada, representa o campo magnético da Terra e os paralelos.

Como no primeiro capítulo, a classificação dos germes de funções $f: \mathbb{R}^{2}, 0 \rightarrow \mathbb{R}, 0$ com $j^{1} f(0,0)=0$, através de mudanças pertencentes ao grupo $\mathcal{A}^{*}$ (mudanças que preservam as folhas da 1-forma $d y$ ) nos leva a modelos suaves para o par desejado. Neste caso a classificação é feita através da indução sobre os jatos de $f$.

\subsection{Classificação}

$\mathrm{Na}$ tabela abaixo temos um resumo da classificação que se encontra neste capítulo.

Teorema 2.1.1 Os únicos germes singulares $\mathcal{A}^{*}$-finitamente determinados estão descritos na tabela a seguir, onde exibimos as formas normais, a $\mathcal{A}^{*}$-codimensão dos germes e o número máximo de singularidades $2_{1}$ que ocorrem em uma deformação genérica. 


\begin{tabular}{|c|c|c|c|}
\hline nome & modelo & $\mathcal{A}^{*}$-codimensão & $\sharp 2_{1}$ \\
\hline $2_{k}$ & $x^{2} \pm y^{k+1}$ & $k-1$ & $k$ \\
\hline
\end{tabular}

A demonstração deste teorema segue do estudo feito neste capítulo.

Proposição 2.1.2 Seja $f: \mathbb{R}^{2}, 0 \rightarrow \mathbb{R}, 0$ um germe na origem com $j^{1} f \equiv 0$. Então as $\mathcal{A}^{*}$ órbitas de $f$ em $J^{2}(2,1)$ sāo

$$
x^{2} \pm y^{2}, x^{2}, x y, y^{2}, 0
$$

Demonstração: Desde que $j^{1} f \equiv 0$ podemos escrever $j^{2} f(x, y)=a_{0} x^{2}+a_{1} x y+a_{2} y^{2}$. Aplicando mudanças em $\mathcal{A}^{*}$ temos

$$
j^{2} f(x, y) \simeq_{\mathcal{A}^{*}} \begin{cases}x^{2} \pm y^{2} & \text { se } a_{1}^{2}-4 a_{0} a_{2} \neq 0, a_{0} \neq 0 \\ x^{2} & \text { se } a_{1}^{2}-4 a_{0} a_{2}=0, a_{0} \neq 0 \\ x y & \text { se } a_{0}=0, a_{1} \neq 0 \\ y^{2} & \text { se } a_{0}=a_{1}=0, a_{2} \neq 0 \\ 0 & \text { se } a_{0}=a_{1}=a_{2}=0\end{cases}
$$

Estudemos cada uma das órbitas de $j^{2} f$.

Proposição 2.1.3 O germe $f(x, y)=x^{2} \pm y^{2}$ é $2-\mathcal{A}^{*}$-determinado e estável.

Demonstração: Dado o germe $f(x, y)=x^{2} \pm y^{2}$ temos

$$
\left\{\begin{array}{l}
f_{x}=2 x \\
f_{y}= \pm 2 y
\end{array}\right.
$$

Sendo assim, de $f_{x}$ temos todos os monômios de grau $k$ em $L \mathcal{A}^{*} \cdot f+\mathcal{M}_{2}^{k+1}$, exceto $y^{k}$. Mas este é gerado através de $f_{y}$. Logo $\mathcal{M}_{2}^{k} \mathcal{E}_{2} \subset L \mathcal{A}^{*} \cdot f+\mathcal{M}_{2}^{k+1}$, para todo $k$. Então pelo Teorema 1.2.5 temos que $f(x, y)=x^{2} \pm y^{2}$ é um germe 2- $\mathcal{A}^{*}$-determinado. Além disso $\mathcal{E}_{2}=L \mathcal{A}_{e}^{*}$, ou seja, o germe $f$ é estável.

Quanto as demais órbitas temos

Proposição 2.1.4 Seja $f: \mathbb{R}^{2}, 0 \rightarrow \mathbb{R}, 0$ tal que $j^{2} f=x^{2}$. As $\mathcal{A}^{*}$-órbitas em $J^{k}(2,1)$ com $j^{k-1} f=x^{2}$ são

$$
x^{2} \pm y^{k}, x^{2}
$$

para todo $k \geq 3$. Ainda mais, $f(x, y)=x^{2} \pm y^{k}$ é um germe $k-\mathcal{A}^{*}$-determinado cujo desdobramento versal é dado por $F\left(x, y, u_{k-2}, \ldots u_{1}\right)=x^{2} \pm y^{k}+u_{k-2} y^{k-2}+\ldots+u_{1} y$. 
Demonstração: Dado o germe $f(x, y)=x^{2}$ temos $f_{x}=2 x$ e $f_{y}=0$, então os monômios da forma $y^{k}$ não pertencem a $L \mathcal{A}^{*} \cdot f$, portanto $f$ não é finitamente $\mathcal{A}^{*}$-determinado.

Estudemos então o $k$-jato dos germes que possuem $(k-1)$-jato igual $x^{2}$. Através do Teorema da Transversal Completa temos $j^{k} f(x, y) \sim \mathcal{A}^{*}\left\{\begin{array}{l}x^{2} \pm y^{k}, \text { ou } \\ x^{2}\end{array}\right.$

Considerando o germe $f(x, y)=x^{2} \pm y^{k}$ temos

$$
\begin{aligned}
& f_{x}=2 x \\
& f_{y}= \pm k y^{k-1} .
\end{aligned}
$$

Então todos os monômios de grau $r>k$ pertencem a $L \mathcal{A}^{*} \cdot f+\mathcal{M}_{2}^{r+1}$. Pelo Teorema 1.2.5, o germe $f$ é $k-\mathcal{A}^{*}$-determinado. Ainda mais, $\mathcal{E}_{2} / L \mathcal{A}_{e}^{*} \cdot f=\mathbb{R}\left\{y, \ldots, y^{k-2}\right\}$, ou seja, este germe é de codimensão $k-2$.

Proposição 2.1.5 Não existe germe $f: \mathbb{R}^{2}, 0 \rightarrow \mathbb{R}, 0$ finitamente $\mathcal{A}^{*}$-determinado tal que $j^{2} f(x, y)=x y$.

Demonstração: Considere o germe $f(x, y)=x y$. Então $f_{x}=y$ e $f_{y}=x$. Conseqüentemente os monômios da forma $x^{k}$ não pertencem a $L \mathcal{A}^{*} \cdot f$, ou seja, este germe não é finitamente $\mathcal{A}^{*}$-determinado. Passando ao 3 -jato de $f$, usando o Teorema da Transversal Completa temos $j^{3} f(x, y) \sim x y+x^{3}$ ou $x y$

Denotando por $f(x, y)=x y+x^{3}$ temos $f_{x}=y+3 x^{2}$ e $f_{y}=x$. Notemos então que os monômios da forma $x^{2 n+1}$ pertencem a $L \mathcal{A}^{*} \cdot f+\mathcal{M}_{2}^{2 n+2}$. Basta considerar os seguintes vetores:

$$
\begin{aligned}
x^{2 n-1} f_{x} & =x^{2 n-1} y+3 x^{2 n+1} \\
& \vdots \\
x^{2 n-1-2 i} y^{i} f_{x} & =x^{2 n-1-2 i} y^{i+1}+3 x^{2 n+1-2 i} y^{i}, \quad(0 \leq i \leq n-1) \\
& \vdots \\
x y^{n-1} f_{x} & =x y^{n}+3 x^{3} y^{n-1} \\
y^{n} f_{y} & =x y^{n} .
\end{aligned}
$$

O mesmo ocorre com os monômios da forma $x^{3 n}$. Considere o seguinte sistema, formado por vetores em $L \mathcal{A}^{*} \cdot f+\mathcal{M}_{2}^{3 n+1}$.

$$
\begin{aligned}
x^{3 n-2} f_{x} & =x^{3 n-2} y+3 x^{3 n} \\
& \vdots \\
x^{3 n-2-2 i} y^{i} f_{x} & =x^{3 n-2-2 i} y^{i+1}+3 x^{3 n-2 i} y^{i} \quad(0 \leq i \leq n-1) \\
& \vdots \\
x^{n} y^{n-1} f_{x} & =x^{n} y^{n}+3 x^{n+2} y^{n-1} \\
f^{n} & =x^{n} y^{n}+\sum_{i=1}^{n-1} C_{n}^{n-i} x^{3 n-2 i} y^{i}
\end{aligned}
$$


onde $C_{n}^{n-i}$ denota o número máximo de possíveis combinações de $n$ elementos formando grupos de $n-i$ elementos. A matriz associada ao sistema acima tem a forma

$$
\left[\begin{array}{cccccc}
3 & 1 & 0 & \ldots & 0 & 0 \\
0 & 3 & 1 & \ldots & 0 & 0 \\
\ldots & \ldots & \ldots & \ldots & \ldots & \ldots \\
0 & 0 & 0 & \ldots & 3 & 1 \\
1 & n & C_{n}^{n-1} & \ldots & n & 1
\end{array}\right]
$$

cujo determinante é dado por $\sum_{i=0}^{n}(-1)^{i} 3^{i} C_{n}^{i}+3^{n}+(-1)^{n} \neq 0$.

Resta-nos então verificar que não é possível gerar os monômios da forma $x^{2 n}$ tal que $2 n \neq 3 k$, para todo $n$ e $k$. Notemos que se $2 n \neq 3 k$ então $2 n=6 k+2$ ou $2 n=6 k+4$, para algum $k$. Mostraremos que por maior que seja o jato considerado, os monômio da forma $x^{2 m}, \operatorname{com} 2 m \neq 3 k$ não podem ser gerados pelo germe considerado, ou seja, dado um germe da forma

$$
f(x, y)=x y+x^{3}+\lambda_{04} x^{4}+\ldots+\lambda_{k 2} x^{6 k+2}+\lambda_{k 4} x^{6 k+4}
$$

todos os monômios da forma $x^{2 m}$, com $m>n=3 k+1,3 k+2$ não pertencem a $L \mathcal{A}^{*} \cdot f+\mathcal{M}_{2}^{2 m+1}$.

Para provar a afirmação acima vamos considerar o conjunto de todos os possíveis vetores em $L \mathcal{A}^{*} \cdot f+\mathcal{M}_{2}^{2 m+1}$ envolvendo os monômios de grau menor ou igual a $2 n$ que não podem ser gerados através de $f_{x}$ como elementos de $T \mathcal{A}^{*} \cdot f+\mathcal{M}_{2}^{2 m+1}$. Mostraremos que para todo par $(m, n)$ de inteiros positivos com $m>n$ obtemos mais monômios do que vetores, portanto o sistema formado pelos vetores descritos acima é impossível.

Observe que dos monômios de grau $2 m$, apenas o monônio $x^{2 m}$ não é gerado como elemento de $T \mathcal{A}^{*} \cdot f+\mathcal{M}_{2}^{2 m+1}$, via $f_{x}$. Dos monômios de grau $2 m-1$ apenas $x^{2 m-1}$ e $x^{2 m-2} y$ não são gerados como vetores de $T \mathcal{A}^{*} \cdot f+\mathcal{M}_{2}^{2 m+1}$, por $f_{x}$. Enquanto que dos monômios de grau $2 m-r$ temos os seguintes monômios nas condições acima : $x^{2 m-r}, x^{2 m-r-1} y, \ldots, x^{2 m-r-i} y^{i}, \ldots, x^{2 m-2 r} y^{r}$. Portanto a partir de $r=m$, com exceção de $x y^{r-j}, j<r$, todos os monômios de grau menor ou igual a $r$ não serão gerados como elementos de $T \mathcal{A}^{*} \cdot f+\mathcal{M}_{2}^{2 m+1}$ via $f_{x}$, portanto serão monômios considerados em nosso sistema. No diagrama a seguir, exibimos todos os monômios considerados em nosso sistema. Note que os monômios $x, y, x^{2}$ não serão considerados pois não pertencem a $T \mathcal{A}^{*} \cdot f$ enquanto que os monômios $x y^{r-j}, j<r$ pertencem a $T \mathcal{A}^{*} \cdot f+\mathcal{M}_{2}^{2 m+1}$ via $f_{y}$, logo também não serão considerados aqui. Lembramos que $m=3 k+1$ ou $m=3 k+2$. 


$$
\begin{aligned}
& x^{2 m} \\
& x^{2 m-1} \quad x^{2 m-2} y \\
& x^{2 m-2} \quad x^{2 m-3} y \quad x^{2 m-4} y^{2} \\
& \text { : } \\
& x^{2 m-r} \quad x^{2 m-r-1} y \quad x^{2 m-r-2} y^{2} \quad x^{2 m-r-3} y^{3} \quad \ldots \quad x^{2 m-2 r} y^{r} \\
& \vdots \\
& \begin{array}{lllllllll}
x^{m} & x^{m-1} y & x^{m-2} y^{2} & x^{m-3} y^{3} & \ldots & \ldots & \ldots & -y^{m}
\end{array} \\
& \begin{array}{lllllll}
x^{m-1} & x^{m-2} y & x^{m-3} y^{2} & x^{m-4} y^{3} & \ldots & \ldots & -y^{m-1}
\end{array} \\
& \text { : } \\
& \begin{array}{rrrr}
x^{3} & x^{2} y & - & y^{3} \\
- & - & y^{2}, &
\end{array}
\end{aligned}
$$

Então o sistema possui $m^{2}+m-2$ monômios.

Enquanto que os vetores em $T \mathcal{A}^{*} \cdot f+\mathcal{M}_{2}^{2 m+1}$, envolvendo os monômios acima, são os seguintes:

$$
\begin{aligned}
& x^{i} f_{x} ; \quad 1 \leq i \leq 2 m-2, \quad \text { geram } \quad x^{t} ; \quad 3 \leq t \leq 2 m \\
& x^{i} y f_{x} ; \quad 0 \leq i \leq 2 m-4, \quad \text { geram } \quad x^{t} y, \quad 2 \leq t \leq 2 m-2 \\
& x^{i} y^{r} f_{x} ; \quad 0 \leq i \leq 2 m-2 r-2, \quad \text { geram } \quad x^{t} y^{r} ; \quad t=0,2 \leq t \leq 2 m-2 r
\end{aligned}
$$

onde $1 \leq r \leq m-1$. E ainda temos os vetores $f^{2}, \ldots, f^{k}$, onde $m=3 k+1$ ou $m=3 k+2$, afinal para $j>k$, todos os monômios que aparecem em $f^{j}$ pertencem a $T \mathcal{A}^{*} \cdot f+\mathcal{M}_{2}^{2 m+1}$. Logo temos $2 m-2+\sum_{r=1}^{m-1}(2 m-2 r-1)+k-1$ vetores, isto é, $m^{2}-1+k-1$ vetores envolvendo as $m^{2}+m-2$ incógnitas. Portanto a diferença entre o número de incógnitas e o número de vetores é igual a $2 k-1$ para $m=3 k+1$ ou $2 k$ para $m=3 k+2$. Sendo $k \geq 1$ temos sempre mais incógnitas do que vetores, isto é um sistema impossível. Assim concluímos que $x^{2 m} \notin T \mathcal{A}^{*} \cdot f+\mathcal{M}_{2}^{2 m+1}$, para todo par $(n, m) \operatorname{com} n<m$. Logo $\mathcal{M}_{2}^{2 n} \not \subset L \mathcal{A}^{*} \cdot f$, o que implica que o germe não é $\mathcal{A}^{*}$-finitamente determinado.

Considerando $f(x, y)=y^{2}$ temos $f_{x}=0$ e $f_{y}=2 y$, logo apenas os monômios da forma $y^{k}$ pertencem a $T \mathcal{A}^{*} \cdot f+\mathcal{M}_{2}^{k+1}$, isto é, o germe $f$ não é finitamente determinado. Mas será que existe um $k$ tal que $j^{k} f$ é finitamente $\mathcal{A}^{*}$ determinado?

A resposta é negativa.

Dado o germe $f$ com $j^{2} f(x, y)=y^{2}$, se existisse germe $g$ tal que $f(x, y)=y^{2}+g(x, y)$ fosse $\mathcal{A}^{*}$-finitamente determinado, teríamos $F_{u}(x, y)=y^{2}+g(x, y)+u x y$ como elemento do desdobramento versal de $f$, pois $x y \notin L \mathcal{A}^{*} \cdot f$, então $F_{u}$ seria $\mathcal{A}^{*}$-finitamente determinado. 
Mas já vimos na Proposição 2.1.5 que germes cujo 2-jato é $x y+y^{2} \sim x y$ não são finitamente $\mathcal{A}^{*}$-determinados, logo $f$ não é germe finitamente $\mathcal{A}^{*}$-determinado.

O mesmo acontece com os germe cujo $j^{2} f=0$, afinal o germe $x y$ sempre será um elemento de seu desdobramento versal. Portanto os únicos germes finitamente $\mathcal{A}^{*}$-determinados sāo dados pela série $f_{k}(x, y)=x^{2} \pm y^{k}$.

Do estudo acima temos o seguinte teorema:

Teorema 2.1.6 Dado um par de germes de 1 -formas diferenciais (dy,df), com $j^{1} f \equiv 0$, os únicos modelos suaves de codimensão finita são

$$
\left(d y, d\left(x^{2} \pm y^{k}\right)\right), \quad k \geq 2,
$$

com codimensão $k-2$. Em particular o único germe estável ocorre para $k=2$. Além disso, um desdobramento do par é dado por $\left(d y, d\left(x^{2} \pm y^{k}+u_{k-2} y^{k-2}+\ldots+u_{1} y\right)\right)$.

\subsection{Estudo geométrico}

Dado o par de germes de 1-formas diferenciais $(d y, d f)$, com $j^{1} f \equiv 0$ temos o conjunto discriminante dado por $\delta(x, y)=f_{x}(x, y)$.

Desde que os únicos pares de germes finitamente $\mathcal{A}^{*}$-determinados são $\left(d y, d\left(x^{2} \pm y^{k}\right)\right)$, temos o discriminante $\delta_{k}(x, y)=2 x=0$, que é uma curva suave para todo $k$. Além disso para $k=2$ temos um germe estável, logo o conjunto de bifurcaçāo é vazio. Ver figura 2.1.
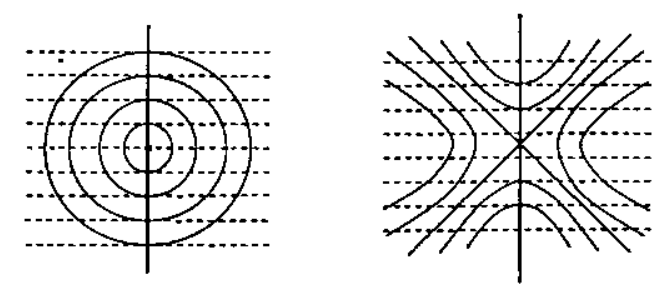

Figura 2.1: Par de 1-formas $\left(d y, d\left(x^{2}+y^{2}\right)\right)$ à esquerda (regular/centro), $\left(d y, d\left(x^{2}-y^{2}\right)\right)$ à direita (regular/sela)

Novamente as deformações do par de germes $(d y, d f)$ são obtidas a partir das deformaçōes do germe $f$. Então vamos estudar a geometria dos desdobramentos versais de $f$.

Neste caso o conjunto de bifurcação do germe $f$ é dado por:

$$
\begin{aligned}
B i f(F) & =\left\{u: \exists x \text { com } f^{u} \mathcal{A}^{*}-\text { instável em } x\right\} \\
& =\left\{u: f^{u} \text { tem singularidade } A_{k}, k>1\right\} \\
& =\left\{u: f_{x}^{u}=f_{y}^{u}=f_{x y}^{2}-f_{x x} f_{y y}=0\right\} .
\end{aligned}
$$

Para $k=3$ observamos que os pares $\left(d y, d\left(x^{2}+y^{3}\right)\right)$ e $\left(d y, d\left(x^{2}-y^{3}\right)\right)$ são suavemente equivalentes e que um desdobramento versal é dado por $\left(d y, d\left(x^{2}+y^{3}+u_{0} y\right)\right)$. Quanto ao 
conjunto de bifurcação, desde que

$$
\begin{aligned}
f_{x} & =2 x ; \quad f_{y}=3 y^{2}+u_{0} ; \\
f_{x x} & =2 ; \quad f_{y x}=0 ; \quad f_{y y}=6 y ;
\end{aligned}
$$

temos $u_{0}=0$, ou seja, o conjunto de bifurcação é a origem. As deformações desse par de germe estão na Figura 2.2.
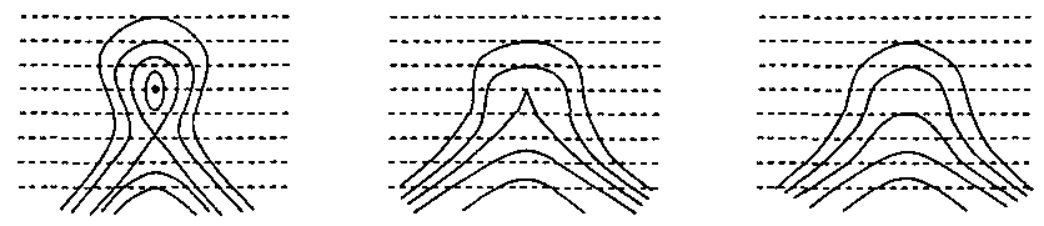

Figura 2.2: Deformações genéricas do par $\left(d y, d\left(x^{2}+y^{3}\right)\right)$

Se $k=4$ temos a família $\left(d y, d\left(x^{2} \pm y^{4}+u_{0} y+u_{1} y^{2}\right)\right)$. Então

$$
\begin{aligned}
f_{x} & =2 x=0 ; \quad f_{y}= \pm 4 y^{3}+2 u_{1} y+u_{0}=0 ; \\
f_{x x} & =2 ; \quad f_{y y}= \pm 12 y^{2}+2 u_{1}=0 \quad f_{x y}=0 .
\end{aligned}
$$

Portanto o conjunto de bifurcação é dado por $\left\{\left(u_{1}, u_{0}\right) \in \mathbb{R}^{2} ; u_{1}=\mp 6 y^{2}, u_{0}= \pm 8 y^{3}\right\}$, uma cúspide. As deformações genéricas desse par estão representadas na Figura 2.3. Novamente observamos que o conjunto de bifurcação divide o conjunto dos parâmetros em duas regiões, onde observamos pares estáveis. Atravessando os ramos do conjunto de bifurcação observamos as deformações genéricas dos pares de codimensão 1.

Vale observar que na Figura 2.3 temos simetria em relação ao eixo $O x$, o que pode ser verificado diretamente pelas equações que as define. Além disso o conjunto de bifurcação tem uma componente multi-local, isto é,

$$
\left\{u: \exists p_{1}, p_{2} \text {, pontos singulares de } f_{u} \text { com } f_{u}\left(p_{1}\right)=f_{u}\left(p_{2}\right)\right\} \text {. }
$$

Afinal $p_{1}, p_{2}$ são pontos singulares de $f$ no mesmo nível se, e somente se, $p_{i}=\left(0, p_{i}^{\prime}\right)$ e os $p_{i}^{\prime}$ são rá́zes duplas de $\pm y^{4}+u y^{2}+v y+w=0$, para algum $w \in \mathbb{R}, i=1,2$, ou seja, $\pm y^{4}+u y^{2}+v y+w=$ $\left(y-p_{1}^{\prime}\right)^{2}\left(y-p_{2}^{\prime}\right)^{2}$. Dessa igualdade temos $p_{1}^{\prime}=-p_{2}^{\prime}$ e $v=0, u=6 p_{1}^{\prime 2}, w=p_{1}^{4}$.

Logo o conjunto dos $u^{\prime}=(u, v)$ tal que existem $p_{1}, p_{2}$ pontos singulares de $f^{u}$ tal que $f^{u}\left(p_{1}\right)=f^{u}\left(p_{2}\right)$ é formado pelos pontos da forma $u^{\prime}=(u, 0)$.

Como no capítulo 1 , restringimos nosso estudo geométrico aos pares de codimensão menor ou igual a 2.

\subsection{Invariantes}

Dado um par de germes de 1-formas $(d y, d f)$ com $f$ singular, temos a $\mathcal{A}^{*}$-codimensão de $f$ e o grau de determinação de $f$ como invariantes mas ainda podemos associar um invariante 

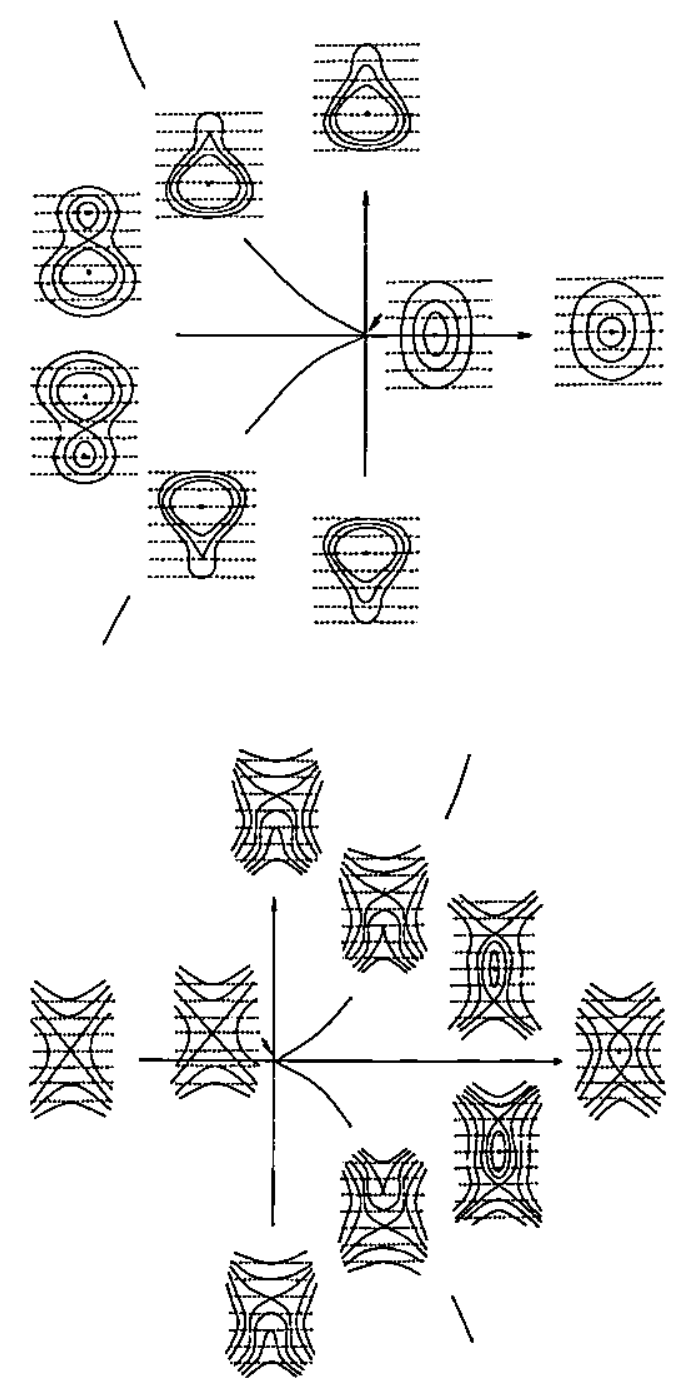

Figura 2.3: Deformaçōes genéricas do par $\left(d y, d\left(x^{2}+y^{4}\right)\right)$ (acima) e do par $\left(d y, d\left(x^{2}-y^{4}\right)\right)$ (embaixo)

geométrico a este par de 1-formas dado pelo tipo de singularidade de $f$, que é definido por

$$
\sharp 2_{1}(d y, d f)=\operatorname{dim}_{\mathbb{R}} \frac{\mathcal{E}_{2}}{<f_{x}, f_{y}>} .
$$

Novamente vamos abusar da notação e escrever $\sharp 2_{1}(f)$ para $\sharp 2_{1}(d y, d f)$.

Esta definição foi motivada pela observação dos desdobramentos versais dos germes $\mathcal{A}^{*}$ finitamente determinados apresentados na seção anterior. Observamos que os pontos singulares de $f$ sempre aparecem em número finito nas deformações versais dos germes mencionados além de aparecer em pontos isolados.

Como no caso anterior, vamos interpretar esse número como o número máximo de pontos que satisfazem o sistema $f_{x}=f_{y}=0$. A proposição abaixo mostra que este é um invariante suave para pares de germes de 1 -formas. 
Proposição 2.3.1. Dados dois germes de aplicações $f, g: \mathbb{R}^{2}, 0 \rightarrow \mathbb{R}, 0 \mathcal{A}^{*}$-equivalentes, então $\sharp 2_{1}(f)=\sharp 2_{1}(g)$.

Demonstração: Se $f \simeq \mathcal{A}^{*} g$ então $g=k \circ f \circ h$ sendo que $h(x, y)=\left(h^{1}(x, y), h^{2}(y)\right), \log$ o

$$
\begin{aligned}
& g_{x}=k^{\prime}(f \circ h) \cdot\left(f_{x} \circ h\right) \cdot h_{x}^{1} \\
& g_{y}=k^{\prime}(f \circ h) \cdot\left(f_{x} \circ h \cdot h_{y}^{1}+f_{y} \circ h \cdot h_{y}^{2}\right)
\end{aligned}
$$

Sendo assim $\frac{\mathcal{E}_{2}}{\left\langle f_{x}, f_{y}\right\rangle}=\mathbb{R} \cdot\left\{\theta_{1}, \theta_{2}, \ldots, \theta_{p}\right\}$, isto é, para todo $H \in \mathcal{E}_{2}$, existem $\lambda_{i} \in \mathbb{R}, i=1,2, \ldots p$ tal que $H=\sum \lambda_{i} \theta_{i}+\varphi_{1} f_{x}+\varphi_{2} f_{y}$.

Então se $e_{1}, e_{2}, e_{3}$ são funções unitárias temos $\frac{\mathcal{E}_{2}}{\left\langle e_{i} f_{x}, e_{2} f_{x}+e_{3} f_{y}\right\rangle}=\mathbb{R} \cdot\left\{\theta_{1}, \theta_{2}, \ldots, \theta_{p}\right\}$, e ainda, $\operatorname{dim}_{\mathbb{R}} \frac{\mathcal{E}_{2}}{\left\langle f_{x} \circ h, f_{y} \circ h\right\rangle}=\operatorname{dim}_{\mathbb{R}} \frac{\mathcal{E}_{2}}{\left\langle f_{x}, f_{y}\right\rangle}$.

Portanto $\operatorname{dim}_{\mathbb{R}} \frac{\mathcal{E}_{2}}{\left\langle f_{x}, f_{y}\right\rangle}=\operatorname{dim}_{\mathbb{R}} \frac{\mathcal{E}_{2}}{\left\langle g_{x}, g_{y}\right\rangle}$.

Observamos que se complexificamos $f$, temos $\operatorname{dim}_{\left.\mathbf{C}<f_{x}, f_{y}\right\rangle}=\mu(f)$. Desde que este invariante coincide com o número de Milnor do germe de aplicação $f$ este é um invariante topológico, conseqüentemente também é suave.

Outro fato que observa-se é que este invariante é sempre atingido por uma deformação real dos pares finitamente $\mathcal{A}^{*}$-determinados, como mostra a proposição abaixo.

Proposição 2.3.2 Dado um par de germes $(d y, d f), f: \mathbb{R}^{2}, 0 \rightarrow \mathbb{R}, 0$ tal que $j^{1} f(0,0)=0$ e $f$ é germe $\mathcal{A}^{*}$-finitamente determinado, temos $\sharp 2_{1}(f)=k-1$ e ainda, existe uma deformação real tal que o número máximo de pontos $2_{1}$ é observado.

Demonstração: Dado o par $(d y, d f)$ nas condiçōes da proposição, podemos escrever $\left(d y, d\left(x^{2} \pm\right.\right.$ $\left.y^{k}\right)$ ). Logo $f_{x}=2 x$ e $f_{y}=k y^{k-1}$ então $\frac{\mathcal{E}_{2}}{\left\langle f_{x}, f_{y}\right\rangle}=\mathbb{R} \cdot\left\{y^{k-2}, \ldots, y, 1\right\}$, ou seja, $\sharp 2_{1}(f)=k-1$.

Além disso, dada a família $\left(d y, d\left(x^{2} \pm y^{k}+u_{k-2} y^{k-2}+\ldots+u_{1} y\right)\right)$, deformação versal de $(d y, d f)$, as soluções do sistema de equações $f_{x}=f_{y}=0$ coincidem com as raízes do polinômio $k y^{k-1}+(k-2) u_{k-2} y^{k-3}+\ldots+u_{1}=0$. Desde que é podemos escolher $u_{k-2}, \ldots, u_{1}$ convenientemente tal que o polinômio acima tenha $k-1$ rázes, o número máximo de pontos $2_{1}$ é atingido por uma deformação real de $f$. 


\section{Capítulo 3}

\section{Classificação suave de pares de folheações $(d y, d f)$ sobre variedades}

\section{de dimensão 2 com bordo regular}

Podemos estudar também pares de folheações definidas sobre uma variedade de dimensão 2 com bordo regular, através da teoria de singularidades. Como nos capítulos anteriores vamos apresentar as formas normais, o grau de determinação, as perturbações versais, a codimensão, estudar a geometria e ainda apresentar invariantes aos pares de folheações definidos por 1-formas diferenciais no semiplano.

Estes pares aparecem no estudo da configuração das linhas de curvatura e das curvas assintóticas em superfícies com bordo.

Exemplo 3.1 Linhas de curvatura

Seja $M$ uma superfície com bordo regular e $p \in M$. As linhas de curvatura de $M$ em $p$ satisfazem a equação diferencial binária dada por

$$
(f E-e F)(x, y) d y^{2}+(g E-e G)(x, y) d x d y+(g F-f G)(x, y) d x^{2}=0
$$

onde $E, F, G, e, f, g$ são os coeficientes da primeira e segunda forma fundamental, respectivamente. Então se p é um ponto não umbilico os coeficientes de 3.1 não são todos nulos, portanto a equação determina duas direções ortogonais no plano, afinal ela pode ser escrita localmente na forma

$$
\left(a_{1}(x, y) d x+a_{2}(x, y) d y\right)\left(b_{1}(x, y) d x+b_{2}(x, y) d y\right)=0 .
$$

para alguns germes de funções suaves $a_{i}, b_{i}, i=1,2$, com $a_{i}$ e $b_{i}$ não todos nulos em $p$. Então a configuração das linhas de curvatura na vizinhança dos pontos não umbilicos é dada pelo estudo dos pares de 1-formas diferenciais no plano. Se p é um ponto umbilico no interior de $M$, temos 
os trabalhos de Sotomayor e Gutierrez, [20] e de Bruce e Fidal, [6], sobre a configuração das linhas de curvatura numa vizinhança do ponto umbilico.

Para estudar o caso da configuração das linhas de curvatura numa vizinhança de um ponto de bordo da superfície $M$, quando o bordo não passa por pontos umbilicos, basta considerar pares de 1 -formas regulares sobre o bordo.

\section{Exemplo 3.2 Curvas assintóticas}

Seja $p$ um ponto de $M$. As curvas assintóticas também satisfazem uma equação diferencial binária dada por

$$
e(x, y) d y^{2}+2 f(x, y) d y d x+g(x, y) d x^{2}=0 .
$$

onde $e, f, g$ são os coeficientes da segunda forma fundamental. Então de acordo com o sinal do discriminante $\delta=f^{2}-$ eg temos duas, uma ou nenhuma direção assintótica. Em pontos elipticos não existem direções assintóticas, desde que o discriminante $\delta$ é negativo, nos pontos parabólicos temos apenas uma direção $(\delta=0)$ e duas direçöes nos pontos hiperbólicos $(\delta>0)$.

Sendo assim, dado um ponto $p \in M$, se $p$ é um ponto elíptico não teremos curvas assintóticas por $p$. Se p é ponto hiperbólico temos um par de campos de direções cujas curvas integrais são as curvas assintóticas e finalmente, se p é um ponto parabólico temos a configuração das curvas assintóticas numa vizinhança de p dada em [4] e [16], veja também [33].

Suponha que desejamos estudar a configuração das curvas assintóticas numa vizinhança de um ponto $p$ de bordo da superfície $M$. Se p pertence a região hiperbólica da superfície a equação 3.3 define um par de 1-formas diferenciais sobre a superfície com bordo regular.

Considere o par $(\alpha, \beta)$ de germes de 1-formas definidas sobre uma variedade de dimensão 2 com bordo regular e $p \in \partial M$. Podemos considerar cartas locais de modo que uma vizinhança de $p$ seja a imagem de $U=\left\{(x, y) \in \mathbb{R}^{2}, 0: y \geq 0\right\}$ por uma carta $\varphi: U \rightarrow M \operatorname{com} \varphi(0,0)=p$. Dessa maneira o bordo é dado por $\phi(y=0)$ e o $\operatorname{par}(\alpha, \beta)$ pode ser estudado em $\left\{(x, y) \in \mathbb{R}^{2}, 0 ; y \geq 0\right\}$.

'Dado o par de folheações $(\alpha, \beta)$ em uma variedade com bordo regular, onde $\alpha$ é regular, vamos considerar dois casos em relação a posição do bordo $y=0$ e a folha de $\alpha$ na origem:

- o bordo é transversal a folha de $\alpha$ na origem (seção 3.1);

- o bordo coincide com uma folha da 1-forma $\alpha$ (seção 3.2);

Nos Exemplos 3.1 e 3.2, o bordo é transversal a folha de $\alpha$ na origem, então estamos no primeiro caso acima.

Neste caso, como $\alpha$ é 1-forma regular podemos supor que $\alpha=d x$ e se $\beta$ é também regular, podemos supor que $\beta=d f$, onde $f: \mathbb{R}^{2}, 0 \rightarrow \mathbb{R}, 0$ é germe diferenciável em $\left\{(x, y) \in \mathbb{R}^{2}, 0\right.$ : $y \geq 0\}$. No caso em que $\beta$ é singular, apenas estudaremos as folheações exatas $(\beta=d f)$. 
Nestas condiçōes para obter as configuraçōes do par $(d x, d f)$ vamos classificar $f$ usando mudanças de coordenadas que preservem as folhas da 1-forma $\alpha$ e o bordo da superfície, ou seja, mudanças de coordenadas na fonte da forma $h(x, y)=\left(h_{1}(x), y h_{2}(x, y)\right)$ e qualquer mudança na meta.

Tais mudanças pertencem a um subgrupo do grupo $\mathcal{A}$, o qual denotamos por $\mathcal{B}(\mathcal{B}$ de bordo). Então $\mathcal{B}$ é dado por $\mathcal{B}=\mathcal{R}^{*} \times \mathcal{L}$, onde $\mathcal{R}^{*}$ é o grupo dos germes de difeomorfismos $h: \mathbb{R}^{2}, 0 \rightarrow \mathbb{R}^{2}, 0$ que preservam as retas verticais $x=c \in \mathbb{R}$ e o bordo $y=0$, isto é $h(x, y)=\left(h_{1}(x), h_{2}(x, y)\right)$, com $h_{1}^{\prime}(0) h_{2}(0,0) \neq 0$ e $h_{2}(0,0)>0$. $\mathcal{L}$ é o grupo dos germes de difeomorfismo na meta. A ação de $\mathcal{B}$ sobre $\mathcal{M}_{2}$ é a ação do grupo $\mathcal{A}$ restrita a $\mathcal{B}$.

Vamos estudar o espaço tangente $L \mathcal{B} \cdot f$, para $f \in \mathcal{M}_{2}$. Desde que o $\mathcal{L}$-espaço tangente é conhecido (ver as preliminares sobre a teoria de singularidades no capítulo 1), resta-nos encontrar o $\mathcal{R}^{*}$-espaço tangente. Para isso considere o seguinte caminho em $\mathcal{R}^{*} \cdot f$

$$
\begin{aligned}
\Phi:(\epsilon,-\epsilon) \times \mathbb{R}^{2} & \rightarrow \mathbb{R} \\
(t, x, y) & \rightarrow f \circ \alpha_{t}(x, y),
\end{aligned}
$$

satisfazendo

$$
\begin{aligned}
\Phi(0, x, y) & =f(x, y) \\
\alpha_{0} & =I d, \\
\alpha_{t}(x, y) & =\left(x+t \alpha_{1}(t, x), y+y t \alpha_{2}(t, x, y)\right)
\end{aligned}
$$

$\operatorname{com} \alpha_{1} \in \mathcal{M}_{x}$ e $\alpha_{2} \in \mathcal{E}_{2}$.

Sendo assim

$$
\left.\frac{\partial \Phi}{\partial t}\right|_{t=0}=\alpha_{1} \frac{\partial f}{\partial x}+\left.y \alpha_{2} \frac{\partial f}{\partial y}\right|_{t=0}
$$

então

$$
L \mathcal{R}_{y}^{*} \cdot f=\mathcal{M}_{x} \frac{\partial f}{\partial x}+y \mathcal{E}_{2} \frac{\partial f}{\partial y}
$$

$\forall f \in \mathcal{M}_{2}$.

Enquanto que

$$
L \mathcal{B} \cdot f=\mathcal{M}_{x}\left\{f_{x}\right\}+\mathcal{E}_{2}\left\{y f_{y}\right\}+f^{*}\left(\mathcal{M}_{u}\right),
$$

onde $\mathcal{M}_{x}$ denota os germes de funções que se anulam na origem e dependem apenas da variável $x$.

Seguindo o estudo feito no capítulo 1 para o grupo $\mathcal{A}^{*}$, podemos demonstrar que $\mathcal{B}$ também é um subgrupo geométrico e portanto temos válido o Teorema da Transversal Completa, assim como o seguinte teorema.

Teorema 3.0.3 [15] Seja $f: \mathbb{R}^{2}, 0 \rightarrow \mathbb{R}, 0$ germe na origem satisfazendo

$$
\mathcal{M}_{2}^{k+1} \subset L \mathcal{B} \cdot f+\mathcal{M}_{2}^{p}
$$

onde $p$ é um polinômio em $k$, independente do germe $f$. Entõo $f$ é $k-\mathcal{B}$-determinado. 
Utilizando a técnica da indução sobre os jatos, já somos capazes de classificar os germes $f$.

\section{1 $\mathcal{B}$-Classificação dos germes $\mathbb{R}^{2}, 0 \rightarrow \mathbb{R}, 0$}

O teorema a seguir apresenta um resumo dos resultados obtidos na $\mathcal{B}$-classificação dos germes $\mathbb{R}^{2}, 0 \rightarrow \mathbb{R}, 0$.

Teorema 3.1.1 Os germes $\mathcal{B}$-finitamente determinados são dados na tabela abaixo, assim como sua codimensão e número máximo de singularidades $\sharp b_{2}$ (sobre o bordo) e $\sharp d_{1}$ que aparecem em uma deformação genérica do germe

\begin{tabular}{|c|l|c|c|c|}
\hline nomes & modelos & $\mathcal{B}_{e}$-codimensão & $\sharp b_{2}$ & $\sharp d_{1}$ \\
\hline$b_{k}^{ \pm}$ & $y \pm x^{k}$ & $k-2$ & $k-1$ & 0 \\
$d_{k}^{ \pm}$ & $x+y^{2} \pm x^{k} y$ & $k-1$ & 0 & $k$ \\
\hline
\end{tabular}

A demonstração deste teorema é feita através de uma sequência de resultados neste capítulo.

Proposição 3.1.2 Seja $f: \mathbb{R}^{2}, 0 \rightarrow \mathbb{R}, 0$ um germe na origem. Então as $\mathcal{B}$-órbitas de $f$ em $J^{1}(2,1) s \tilde{o} o$

$$
y+x, \quad y, \quad x, \quad 0 .
$$

Demonstração: Dada $f$ podemos escrevê-la na forma

$$
f(x, y)=\psi(x)+y \phi(x, y)
$$

Sendo assim temos dois casos a considerar:

(i) $\phi(0,0) \neq 0$,

(ii) $\phi(0,0)=0$.

Se (i) ocorre, podemos fazer mudanças escalares de modo que $f(x, y)=\psi(x)+y(1+\varphi(x, y))$. Fazendo $X=x, Y=y(1+\varphi(x, y))$ temos $f$ suavemente equivalente ao germe $y+\psi(x)$. Sendo assim, $j^{1} f(x, y)=y$ ou $j^{1} f(x, y)=y+x$, de acordo com o $j^{1} \psi$.

Se (ii) ocorre, temos $j^{1} f(x, y)=x$ ou $j^{1} f(x, y)=0$, dependendo do $j^{1} \psi(x)$.

Sendo assim as únicas órbitas em $J^{1}(2,1)$ são $x, y, y+x, 0$.

Vamos estudar cada órbita no $j^{1} f$.

Proposição 3.1.3 O germe $f(x, y)=y+x$ é $1-\mathcal{B}$-determinado e estável. 
Demonstração: Considerando o germe $f(x, y)=y+x$ temos.

$$
\begin{aligned}
f_{x} & =1 \\
y f_{y} & =y .
\end{aligned}
$$

De $f_{x}$ temos $x^{k}$, para todo $k \geq 2$, enquanto que, a partir de $y f_{y}$ temos os monômios da forma $x^{k-j} y^{j}$, para todo $k \geq 2$ e $1 \leq j \leq k$. Logo concluímos que

$$
\mathcal{M}_{2}^{r} \subset L \mathcal{B} \cdot f+\mathcal{M}_{2}^{r+1}, \forall r \geq 2 .
$$

Pelo Teorema 3.0.3, temos que o germe $f(x, y)=y+x$ é $1-\mathcal{B}$-determinado. Além disso temos $L \mathcal{B}_{e} \cdot f=\mathcal{E}_{2}$, logo o germe tem codimensão nula, ou seja, é estável.

Proposição 3.1.4 Seja $f: \mathbb{R}^{2}, 0 \rightarrow \mathbb{R}, 0$ tal que $j^{1} f(x, y)=y$. As $\mathcal{B}$-órbitas em $J^{k}(2,1)$ com $j^{k-1} f(x, y)=y$, são

$$
y \pm x^{k}, \quad y
$$

para todo $k \geq 2$. Além disso, o germe $f(x, y)=y \pm x^{k}$ é $k-\mathcal{B}$-determinado com $\mathcal{B}_{e}$-codimensão $k-2$. Um desdobramento versal deste germe é dado por

$$
F\left(x, y, u_{k-2}, \ldots u_{1}\right)=y \pm x^{k}+u_{k-2} x^{k-2}+\ldots+u_{1} x .
$$

Demonstração: Usando o Teorema da Transversal Completa temos que se um germe possui $j^{k-1} f(x, y)=y$, então $j^{k} f(x, y) \simeq_{\mathcal{B}} y \pm a x^{k}$, para todo $k \geq 2$. Aplicando mudanças escalares temos

$$
j^{k} f(x, y) \simeq_{\mathcal{B}}= \begin{cases}y \pm x^{k}, & \text { se } a \neq 0, \\ y, & \text { caso contrário. }\end{cases}
$$

Denotando por $f$ o germe $y \pm x^{k}$ temos $f_{x}= \pm k x^{k-1}$ e $y f_{y}=y$. Portanto de $y f_{y}$ obtemos os todos monômios de grau $r \geq k+1$, exceto $x^{r}$. O monômio $x^{r}$ por sua vez é obtido de $f_{x}$. Concluímos assim que

$$
\mathcal{M}_{2}^{r} \subset L \mathcal{B} \cdot f+\mathcal{M}_{2}^{r+1}, \quad \forall r \geq k+1
$$

Então o Teorema 3.0.3 nos garante que o germe $f(x, y)=y \pm x^{k}$ é $k-\mathcal{B}$-determinado. Quanto a $\mathcal{B}_{e}$-codimensão de $f$ temos $\mathcal{E}_{2} / L \mathcal{B}_{e}=\mathbb{R} \cdot\left\{x, x^{2}, \ldots, x^{k-2}\right\}$, isto é, a $\mathcal{B}_{e}$-codimensão de $f$ é $k-2$. Com isso o desdobramento versal deste germe é dado por

$$
f\left(x, y, u_{k-2}, \ldots, u_{1}\right)=y \pm x^{k}+u_{k-2} x^{k-2}+\ldots+u_{1} x .
$$

Observação 3.1.5 Vale observar que os germes $f(x, y)=y+x^{k}$ e $g(x, y)=y-x^{k}$ não são germes $\mathcal{B}$-equivalentes se $k$ é par. Considere $k=2 s$ as seguintes as curvas de nivel, $f^{-1}(0) e$ $g^{-1}(0)$ em $\left\{(x, y) \in \mathbb{R}^{2} ; y \geq 0\right\}$. A primeira é um ponto, a origem, enquanto que a segunda é uma curva pela origem. No caso $k$ ímpar os germes são equivalentes. 
Proposição 3.1.6 Seja $f: \mathbb{R}^{2}, 0 \rightarrow \mathbb{R}, 0$ tal que $j^{1} f(x, y)=x$. As B-órbitas em $J^{2}(2,1)$ cujo $j^{1} f(x, y)=x$ sāo

$$
x+x y+y^{2}, \quad x \pm y^{2}, \quad x+x y, \quad x
$$

Demonstração: Pelo Teorema da Transversal Completa, podemos considerar o 2-jato de $f$ na forma

$$
j^{2} f(x, y)=x+a x y+b y^{2} .
$$

Através de mudanças de coordenadas, o 2-jato de $f$ pode ser escrito na forma

$$
j^{2} f(x, y) \simeq_{\mathcal{B}} \begin{cases}x+x y+y^{2}, & \text { se } a \neq 0 \neq b \\ x \pm y^{2}, & \text { se } a=0, b \neq 0 \\ x+x y, & \text { se } b=0, a \neq 0 \\ x, & \text { se } a=b=0 .\end{cases}
$$

Nosso próximo passo, seguindo a indução sobre os jatos, é classificar os germes cujo o 2-jatos são as órbitas acima.

Proposição 3.1.7 O germe $f(x, y)=x+x y+y^{2}$ é $2-\mathcal{B}$-determinado e estável.

Demonstração: Seja $f(x, y)=x+x y+y^{2}$, então temos

$$
\begin{aligned}
f_{x} & =1+y \\
y f_{y} & =x y+2 y^{2} .
\end{aligned}
$$

Usando $f_{x}$ temos $x^{k}$, para todo $k \geq 1$. Para os demais monômios considere os vetores abaixo, pertencentes a $L \mathcal{B} \cdot f+\mathcal{M}_{2}^{k+1} \mathcal{E}_{2}$ :

$$
\begin{aligned}
y^{k-2}\left\{y f_{y}\right\} & =x y^{k-1}+2 y^{k} \\
\vdots & \\
x^{i} y^{k-(i+2)}\left\{y f_{y}\right\} & =x^{i+1} y^{k-(i+1)}+2 x^{i} y^{k-i}
\end{aligned}
$$

com $0 \leq i \leq k-2$.

Fazendo $i=k-2$ construímos o seguinte sistema:

$$
\begin{aligned}
x^{k-2}\left\{y f_{y}\right\} & =x^{k-1} y+2 x^{k-2} y^{2} \\
x^{k-1}\left\{f_{x}\right\} & =x^{k-1}+x^{k-1} y \\
f^{k-1} & \simeq_{\mathcal{B}} x^{k-1}+(k-1) x^{k-1} y+(k-1) x^{k-2} y^{2}
\end{aligned}
$$

para verificar que as demais parcelas de $f^{k-1}$ pertencem a $L \mathcal{B} \cdot f+\mathcal{M}_{2}^{k+1}$ basta usar y $f_{y}$, como fizemos na Proposição 1.3.4. 
A matriz associada ao sistema acima é

$$
\left(\begin{array}{ccc}
0 & 1 & 2 \\
1 & k-1 & k-1 \\
1 & 1 & 0
\end{array}\right)
$$

cujo determinante é não nulo para todo $k$ inteiro positivo. Voltando através das equações em (3.6) temos $\mathcal{M}_{2}^{k} \subset L \mathcal{B} \cdot f+\mathcal{M}_{2}^{k+1}$, para todo $k \geq 3$, logo $f(x, y)=x+x y+y^{2}$ é um germe $2-\mathcal{B}$-determinado.

Além disso este é um germe estável, afinal sua $\mathcal{B}_{e}$-codimensão é nula.

Considere o germe $f(x, y)=x \pm y^{2}$. Este germe não é finitamente $\mathcal{B}$-determinado, afinal os monômios da forma $x^{k} y$, com $k \geq 3$, não pertencem a $L \mathcal{B} \cdot f$, para todo $k \geq 1$.

Seguindo o processo indutivo vamos considerar o $k$-jato cujo $(k-1)$-jato é $x \pm y^{2}$, para todo $k \geq 3$.

Proposição 3.1.8 Seja $f: \mathbb{R}^{2}, 0 \rightarrow \mathbb{R}, 0$ tal que $j^{2} f(x, y)=x \pm y^{2}$. As $\mathcal{B}$-órbitas em $J^{k+1}(2,1)$ cujo $j^{k} f(x, y)=x \pm y^{2}$, para $k \geq 2$, são dadas por

$$
x \pm y^{2}+x^{k} y, \quad x \pm y^{2}
$$

Além disso o germe $f(x, y)=x \pm y^{2}+x^{k} y$ é $(k+1)-\mathcal{B}$-determinado, com $\mathcal{B}_{e}$-codiménsão $k-1$ e desdobramento versal dado por

$$
F\left(x, y, u_{k-2}, \ldots, u_{0}\right)=x \pm y^{2}+y\left(x^{k}+u_{k-2} x^{k-2}+\ldots+u_{2} x^{2}+u_{1} x+u_{0}\right) .
$$

Demonstração: Pelo Teorema da Transversal Completa, dado um germe cujo $j^{k} f(x, y)=x \pm y^{2}$ temos que $j^{k+1} f(x, y) \simeq_{\mathcal{B}} x \pm y^{2}+a x^{k} y$, para todo $k \geq 3$, este por sua vez é $\mathcal{B}$-equivalente ao germe

$$
\begin{cases}x \pm y^{2}+x^{k} y, & \text { se } a \neq 0 \\ x \pm y^{2}, & \text { caso contrário. }\end{cases}
$$

Ainda denotando por $f$ o germe $x \pm y^{2}+x^{k} y$, temos

$$
\begin{aligned}
f_{x} & =1+k x^{k-1} y \\
y f_{y} & = \pm 2 y^{2}+x^{k} y
\end{aligned}
$$

Sendo assim, de $f_{x}$ temos os monômios da forma $x^{r}$, para todo inteiro positivo $r>k+1$ e de $y f_{y}$, os monômios da forma $y^{r}, y^{r-1} x, \ldots, y^{2} x^{r-2}$. Então resta-nos analisar se os monômios da forma $x^{r-1} y$, para todo $r>k+1$, também pertencem a $L \mathcal{B} \cdot f+\mathcal{M}_{2}^{r+1}$. Considere os seguintes vetores:

$$
\begin{aligned}
x^{r-k}\left\{f_{x}\right\} & =x^{r-k}+k x^{r-1} y \\
x^{r-k-1}\left\{y f_{y}\right\} & = \pm 2 x^{r-k-1} y^{2}+x^{r-1} y \\
f^{r-k} & \simeq_{\mathcal{B}} x^{r-k} \pm(r-k) x^{r-k-1} y^{2}+(r-k) x^{r-1} y
\end{aligned}
$$


(como nos casos anteriores as demais parcelas de $f^{r-k}$ pertencem a $L B \cdot f+\mathcal{M}_{2}^{r+1}$ ). A matriz associada ao sistema de equaçōes acima é dada por

$$
\left(\begin{array}{ccc}
1 & k & 0 \\
0 & 1 & \pm 2 \\
1 & \pm(r-k) & r-k .
\end{array}\right)
$$

cujo determinante é não nulo. Sendo assim temos $\mathcal{M}_{2}^{r} \subset L \mathcal{B} \cdot f+\mathcal{M}_{2}^{r+1}$, para todo $r \geq k+2$. Pelo Teorema 3.0.3 o germe $f(x, y)=x \pm y^{2}+x^{k} y$ é $(k+1)-\mathcal{B}$-determinado. Quanto a codimensão deste germe temos que

$$
\frac{\mathcal{E}_{2}}{L \mathcal{B}_{e} \cdot f}=\mathbb{R} \cdot\left\{x^{k-2} y, \ldots, x y, y\right\}
$$

logo o germe tem $\mathcal{B}_{e}$-codimensão $(k-1)$ e um desdobramento versal é dado por

$$
F\left(x, y, u_{k-2}, \ldots, u_{0}\right)=x \pm y^{2}+y\left(x^{k}+u_{k-2} x^{k-2}+\ldots+u_{2} x^{2}+u_{1} x+u_{0}\right)
$$

Considere agora o germe $f(x, y)=x+x y$, ele não é $\mathcal{B}$-determinado, afinal os monômios da forma $y^{k}$, para $k \geq 3$ não pertencem a $L \mathcal{B} \cdot f$.

Aplicando o Teorema da Transversal Completa, temos o 3-jato da forma

$$
j^{3} f(x, y) \simeq_{\mathcal{B}} x+x y+a y^{3} \simeq_{\mathcal{B}} \begin{cases}x+x y+y^{3}, & \text { se } a \neq 0 \\ x+x y, & \text { caso contrário }\end{cases}
$$

Vamos então estudar a determinação do germe $f(x, y)=x+x y+y^{3}$. Veremos que este germe não é $\mathcal{B}$-finitamente determinado, isto é, $\mathcal{M}_{2}^{k} \not \subset L \mathcal{B} \cdot f$, para todo inteiro positivo $k$.

Proposiçāo 3.1.9 Todo germe de função $f: \mathbb{R}^{2}, 0 \rightarrow \mathbb{R}, 0$ com $j^{3} f(x, y)=x+x y+y^{3}$ não é $\mathcal{B}$-finitamente determinado.

Demonstraçăo: Observe que dado o germe $f(x, y)=x+x y+y^{3}$, temos

$$
\begin{aligned}
f_{x} & =1+y \\
y f_{y} & =x y+3 y^{3}
\end{aligned}
$$

logo a única maneira de obter o monômio $y^{4}$ como elementos de $L \mathcal{B} \cdot f$ é através de $y\left\{y f_{y}\right\}=$ $x y^{2}+3 y^{4}$, portanto o germe $f$ não é 3 - $\mathcal{B}$-determinado.

Passando ao 4-jato dos germes que possuem 3-jato dado por $x+x y+y^{3}$ pelo Teorema da Transversal Completa, basta considerarmos o germe $F(x, y)=x+x y+\lambda x y^{2}+y^{3}$. Note que $\lambda$ não pode ser eliminado por mudanças pertencentes ao grupo $\mathcal{B}$, logo $\lambda$ é um módulo suave. 
Observe ainda que para obter alguma informação sobre a determinação finita do germe $F$ com respeito ao grupo $\mathcal{B}$, basta estudarmos os monômios da forma $y^{n}$, para $n>4$, afinal os demais monômios em $\mathcal{M}_{2}^{n}$ podem ser gerados em $L \mathcal{B} \cdot F+\mathcal{M}_{2}^{n+1}$ usando $y f_{y}$.

Não é difícil verificar que $y^{2 n-1} \in L \mathcal{B} \cdot F+\mathcal{M}_{2}^{2 n}$ para todo $n>2$ inteiro positivo, basta considerar os seguintes vetores em $L \mathcal{B} \cdot F+\mathcal{M}_{2}^{2 n}$ :

$$
\begin{aligned}
x^{i} y^{2 n-4-2 i}\left\{y F_{y}\right\} & =x^{i+1} y^{2 n-3-2 i}+2 \lambda x^{i+1} y^{2 n-2-2 i}+3 x^{i} y^{2 n-1-2 i}, \quad 0 \leq i \leq n-2 \\
x^{n-1} F_{x} & =x^{n-1}+x^{n-1} y+\lambda x^{n-1} y^{2} \\
F^{n-1} & \simeq_{\mathcal{B}} x^{n-1}+(n-1) x^{i+1} y+(n-1) x^{n-2} y^{3}
\end{aligned}
$$

Quanto aos monômios da forma $x^{i+1} y^{2 n-2-2 i}$, para todo $0 \leq i \leq n-2$, estes pertencem a $L \mathcal{B} \cdot F+\mathcal{M}_{2}^{2 n}$ (use $y F_{y}$ sucessivamente), assim como os demais monômios em $F^{n-1}$.

Desde que a matriz associada ao sistema formado pelos três últimos vetores em (3.8) possui determinante não nulo, temos por recorrência que $y^{2 n-1} \in L \mathcal{B} \cdot F+\mathcal{M}_{2}^{2 n}$, para todo inteiro positivo $n>3$.

Por outro lado, verificaremos que para todo inteiro $m \geq 2$, o germe $F(x, y)=x+x y+$ $\lambda x y^{2}+y^{3}+\lambda_{3} y^{6}+\ldots+\lambda_{m} y^{2 m}$ não é $\mathcal{B}$-finitamente determinado, afinal os monômios da forma $y^{2 n}, \operatorname{com} n>m$ inteiro positivo, não pertencem a $L \mathcal{B} \cdot F+\mathcal{M}_{2}^{2 n+1}$.

Para isso considere todos os possiveis vetores pertencentes a $L \mathcal{B} \cdot F+\mathcal{M}_{2}^{2 n+1}$ envolvendo os monômios de grau menor ou igual a $2 n$ que não podem ser gerados através de $y F_{y}$ como elementos de $L \mathcal{B} \cdot F+\mathcal{M}_{2}^{2 n+1}$. Mostraremos que para todo par $(m, n)$ de inteiros positivos com $n>m$ obtemos mais monômios do que vetores, portanto o sistema formado pelos vetores $\mathrm{e}$ monômios acima é impossível.

No $2 n$-jato, apenas o monônio $y^{2 n}$ não é gerado como elemento de $L \mathcal{B} \cdot F+\mathcal{M}_{2}^{2 n+1}$, via $y F_{y}$. No $(2 n-1)$-jato temos os monômios $y^{2 n-1}$ e $x y^{2 n-2}$, que não são gerados como vetores em $L \mathcal{B} \cdot F+\mathcal{M}_{2}^{2 n+1}$. Sendo que no $(2 n-r)$-jato temos os monômios $y^{2 n-r}, x y^{2 n-r-1}, \ldots, x^{i} y^{2 n-r-i}, \ldots$, $x^{r} y^{2 n-2 r}$, nas condições acima. Portanto a partir de $r=n$ todos os monômios de grau menor ou igual a $r$ não serão gerados como elementos de $L \mathcal{B} \cdot F+\mathcal{M}_{2}^{2 n+1}$ via $y F_{y}$, logo serão monômios considerados em nosso sistema. Os monômios y $4 y^{2}$ não serão considerados em nosso sistema pois não pertencem a $L \mathcal{B} \cdot F+\mathcal{M}_{2}^{2 n+1}$. Com isso temos um sistema com $n^{2}+2 n-2$ monômios. No diagrama abaixo exibimos todos os monômios envolvidos neste sistema: 


$$
\begin{array}{lllllll}
y^{2 n} & & & & & \\
y^{2 n-1} & x y^{2 n-2} & & & & & \\
y^{2 n-2} & x y^{2 n-3} & x^{2} y^{2 n-4} & & & & \\
\vdots & & & & & & \\
y^{2 n-r} & x y^{2 n-r-1} & \ldots & \ldots & \ldots & \ldots & x^{r} y^{2 n-2 r} \\
\vdots & & & & & & \\
y^{n} & x y^{n-1} & \ldots & \ldots & \ldots & \ldots & x^{n} \\
y^{n-1} & x y^{n-2} & \ldots & \ldots & \ldots & x^{n-1} & \\
\vdots & & & & & & \\
y^{3} & x y^{2} & x^{2} y & x^{3} & & & \\
- & x y & x^{2} & & & & \\
- & x & & & & &
\end{array}
$$

Passando aos vetores em $L \mathcal{B} \cdot F+\mathcal{M}_{2}^{2 n+1}$, gerados em $y F_{y}$, que envolvem os monômios acima temos no $2 n$-jato:

$$
\begin{array}{ll}
y^{2 n-2 i} & \left\{y F_{y}\right\}, \quad 3 \leq i \leq m \\
y^{2 n-3} & \left\{y F_{y}\right\}
\end{array}
$$

No $(2 n-1)$-jato temos

$$
\begin{aligned}
y^{2 n-2 i-1} & \left\{y F_{y}\right\}, \quad 3 \leq i \leq m \\
y^{2 n-4} & \left\{y F_{y}\right\} \\
& \\
x y^{2 n-2 i-2} & \left\{y F_{y}\right\}, \quad 3 \leq i \leq m \\
x y^{2 n-5} & \left\{y F_{y}\right\} .
\end{aligned}
$$

E finalmente, no $(2 n-r)$-jato, para $2 \leq r \leq n-m$

$$
\begin{aligned}
y^{2 n-2 m-r} & \left\{y F_{y}\right\} \\
x^{i} y^{2 n-2 m-r-i} & \left\{y F_{y}\right\}, \quad 1 \leq i \leq r-3 \\
x^{r-2} y^{2 n-2 r-2 m+2} & \left\{y F_{y}\right\} \\
x^{r-2} y^{2 n-2 r-1} & \left\{y F_{y}\right\} \\
x^{r-1} y^{2 n-2 r-2 i+1} & \left\{y F_{y}\right\}, \quad 3 \leq i \leq m \\
x^{r-1} y^{2 n-2 r-2} & \left\{y F_{y}\right\} \\
x^{r} y^{2 n-2 r-2 i} & \left\{y F_{y}\right\}, \quad 3 \leq i \leq m \\
x^{r} y^{2 n-2 r-3} & \left\{y F_{y}\right\}
\end{aligned}
$$


Para $r=n-m+t$, os monômios $x^{i} y^{2 n-r-i}$ não podem ser gerados na última parcela de $y F_{y}$, logo temos um número menor de equações. São elas:

$$
\begin{aligned}
x^{i} y^{n-m-t-i} & \left\{y F_{y}\right\}, \quad 0 \leq i \leq n-m-t, \quad 1 \leq t \leq n-m \\
x^{n-m+t-2} y^{2 m-2 t-1} & \left\{y F_{y}\right\} \\
x^{n-m+t-2} y^{2 m-2 t-2} & \left\{y F_{y}\right\} \\
x^{n-m+t-1} y^{2 m-2 t+1-2 i} & \left\{y F_{y}\right\}, \quad 3 \leq i \leq m-t \\
x^{n-m+t} y^{2 m-2 t-3} & \left\{y F_{y}\right\} \\
x^{n-m+t} y^{2 m-2 t-2 i} & \left\{y F_{y}\right\}, \quad 3 \leq i \leq m-t
\end{aligned}
$$

Quando $r=n-2$, estamos no $n+2$-jato, onde temos os seguintes vetores pertencentes a $L \mathcal{B} \cdot F+\mathcal{M}_{2}^{2 n+1}$ :

$$
\begin{array}{rr}
x^{n-2} y & \left\{y F_{y}\right\} \\
x^{n-3} y^{2} & \left\{y F_{y}\right\} \\
x^{n-4} y^{3} & \left\{y F_{y}\right\}
\end{array}
$$

para os demais monômios neste jato, os vetores já foram considerados anteriormente.

Quando $r=n-1$ temos os vetores

$$
\begin{aligned}
x^{n-3} y & \left\{y F_{y}\right\} \\
x^{n-2} & \left\{y F_{y}\right\}
\end{aligned}
$$

Sendo que para $r \geq n$ todos os vetores envolvendo os moôômios desejados já foram considerados usando $y F_{y}$, resta apenas os vetores

$$
F^{n-1}, F^{n-2}, \ldots, F^{2}
$$

e

$$
x^{n-1} F_{x}, x^{n-2} F_{x}, \ldots, x^{2} F_{x}, x F_{x}
$$

Com isso temos que o número de vetores envolvendo os $n^{2}+2 n-2$ monômios desejados é $n^{2}-n$. A diferença entre o número de monômios e o número de vetores é $3 n-2$. Desde que $n$ é um inteiro positivo estritamente maior que 2, sempre teremos mais monômios do que vetores pertencentes a $L \mathcal{B} \cdot F+\mathcal{M}_{2}^{2 n+1}$ em nosso sistema. Portanto o sistema é impossível.

Concluímos assim que os monômios da forma $y^{2 n} \notin L \mathcal{B} \cdot F+\mathcal{M}_{2}^{2 n+1}$, para todo $n \geq 2$, independente do valor de $m$. Isto é $y^{2 n} \notin L \mathcal{B} \cdot f$, o que é equivalente a dizer que o germe $f(x, y)=x+x y+y^{3}$ não é finitamente $\mathcal{B}$-determinado, afinal não existe inteiro $k$ tal que $\mathcal{M}_{2}^{k} \subset L \mathcal{B} \cdot f$.

Além disso não existem outros germes finitamente $\mathcal{B}$-determinados além daqueles já citados acima, como segue da seguinte proposição: 
Proposição 3.1.10 Seja $f: \mathbb{R}^{2}, 0 \rightarrow \mathbb{R}, 0$ cujo 3-jato é dado por $x+x y$, ou cujo 2-jato é $x$, ou ainda cujo 1-jato é nulo ( $\beta$ é singular). Nenhum desses germes é $\mathcal{B}$-finitamente determinado.

Demonstração: Vamos analisar cada caso separadamente. Primeiramente suponha que $j^{3} f(x, y)=x+x y$. Então $j^{4} f(x, y) \simeq_{\mathcal{B}} x+x y+a y^{4}$, portanto $f_{x}=1+y$ e $y f_{y}=x y+4 a y^{4}$ e o monômio $y^{3}$ não pertence a $L \mathcal{B}_{e} \cdot f$, sendo assim, $f_{u}(x, y)=x+x y+u y^{3}+a y^{4}$ é elemento do $\mathcal{B}$-desdobramento do germe $f$. Mas vimos pela Proposição 3.1 .9 que este não é um germe $\mathcal{B}$-finitamente determinado. Portanto o Lema 1.3.6 assegura que o germe $f(x, y)=x+x y+a y^{4}$ não é $\mathcal{B}$-finitamente determinado. Note ainda que isso acontece para todo $j^{k} f=x+x y$, com $k \geq 3$.

Considere agora o germe $j^{2} f(x, y)=x$. Neste caso $j^{3} f(x, y) \simeq_{\mathcal{B}} x+a x^{2} y+b x y^{2}+c y^{3}$ e denotando o por $f$ temos $f_{x}=1+2 a x y+b y^{2}$ e $y f_{y}=a x^{2} y+2 b x y^{2}+3 c y^{3}$. Sendo assim o monômio $x y$ não pertence a $L \mathcal{B}_{e} \cdot f$ e $f_{u}(x, y)=x+u_{1} x y+c y^{3}+a x^{2} y+b x y^{2}$ é um elemento do $\mathcal{B}$ desdobramento do germe $f$. A Proposição 3.1.9 mostrou-nos que este germe não é $\mathcal{B}$-finitamente determinado, logo pelo Lema 1.3.6 concluímos que o mesmo é válido para o germe cujo 2-jato é $x$. Novamente esse procedimento pode ser feito para todo $k$-jato, com $k \geq 2$, mostrando que não existe germe $\mathcal{B}$-finitamente determinado com $j^{k} f=x$, para todo $k \geq 2$.

Quanto ao germe $j^{1} f=0$, o procedimento é análogo. Temos $j^{2} f(x, y) \simeq_{\mathcal{B}} a x^{2}+b x y+c y^{2}$, portanto os monômios $x, x y$ e $y^{3}$ não pertencem ao espaço tangente estendido. Sendo assim irão ser parcelas das deformações do germe $f$. Novamente teremos um germe como na Proposição 3.1 .9 e do Lema 1.3 .6 concluímos que não existe germe $\mathcal{B}$-finitamente determinado cujo $k$-jato é nulo para $k \geq 1$.

Observação 3.1.11 Da proposição anterior temos que a $\mathcal{B}$-classificação dos germes $\mathbb{R}^{2}, 0 \rightarrow \mathbb{R}, 0$ de codimensão finita obtida é completa.

\subsubsection{Classificação dos pares de folheaçōes}

Dada a classificação anterior podemos enunciar a seguinte proposição sobre pares de folheações.

Proposição 3.1.12 Sejam $\alpha$ e $\beta$ dois germes de 1-formas diferenciais regulares definidas em uma variedade de dimensão 2 com bordo regular. Se a folha da 1-forma $\alpha$ na origem é transversal ao bordo da variedade, temos as seguintes casos:

(i) Se $\alpha$ e $\beta$ são transversais temos $(\alpha, \beta)$ suavemente equivalente a

- $(d x, d(y+x))$, quando a folha de $\beta$ pela origem é transversal ao bordo. Par estável.

- $b_{k}^{ \pm}:\left(d x, d\left(y \pm x^{k}\right)\right.$, quando a folha de $\beta$ pela origem tem contato $k \geq 2$ com o bordo. Um desdobramento versal é dado por $\left(d x, d\left(y \pm x^{k}+u_{k-2} x^{k-2}+\ldots+u_{1} x\right)\right)$. 
(ii) Se as folhas de $\alpha$ e $\beta$ pela origem têm contato 2 entre si e são ambas transversais ao bordo, o par $(\alpha, \beta)$ é equivalente a

- $d_{2 s}^{ \pm}:\left(d x, d\left(x+x^{2 s} y \pm y^{2}\right)\right), o u$

- $d_{2 s-1}:\left(d x, d\left(x+x^{2 s-1} y-y^{2}\right)\right)$

(temos contato $k=2 s$ ou $2 s-1$ entre o discriminante $e$ o bordo). Um desdobramento versal do par é dado por $\left(d x, d\left(x \pm y^{2}+y\left(u_{1}+u_{2} x+\ldots+u_{k-1} x^{k-1}+x^{k}\right)\right)\right.$.

(iv) Se as folhas de $\alpha$ e $\beta$ pela origem têm contato $\geq 3$, não existem modelos suaves de codimensão finita para o par $(\alpha, \beta)$.

Na Figura 3.1 temos os pares estáveis, onde com exceçāo da figura no canto inferior direito, as folheações são transversais. Nas Figuras $3.2--3.5$ temos as deformaçöes dos pares de $\mathcal{B}_{e^{-c o d i m e n s} \tilde{a} o} 1$ e 2 .

Observação 3.1.13 Nas figuras a seguir as retas tracejadas são as folhas da 1-forma dx, as curvas em negrito são o discriminante e o bordo e as demais curvas são as folhas da 1-forma $d f$.

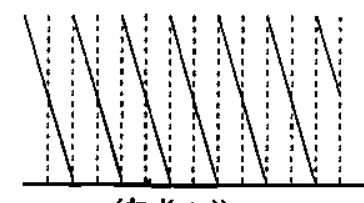

$(d x . d(y+x))$

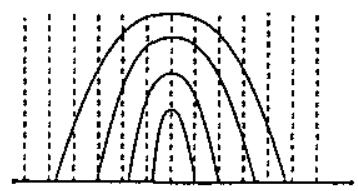

$\left(d x, d\left(y+x^{2}\right)\right)$

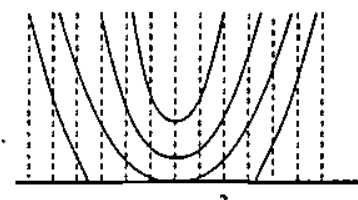

$\left(d x, d\left(y-x^{2}\right)\right)$

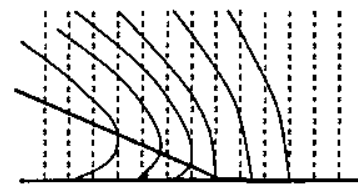

$\left(d x, d\left(x+x y+y^{2}\right)\right)$

Figura 3.1: Pares estáveis

Demonstração: Como vimos no início deste capítulo, podemos supor que $\alpha=d x$ e $\beta=d f$, com $f: \mathbb{R}^{2}, 0 \rightarrow \mathbb{R}, 0$ um germe em $\{(x, y) ; y \geq 0\}$, ou seja, as folhas de $\beta$ são as curvas de nível do germe $f$. Nestas condições temos

$$
\delta(x, y)=f_{y}(x, y)
$$

Então se $\alpha$ e $\beta$ são transversais temos $f_{y}(x, y) \neq 0, \forall(x, y) \in \mathbb{R}^{2}, 0$, o que implica que $j^{1} f(x, y)=y$ ou $y+x$. Então pelas Proposições 3.1.3 e 3.1.4 temos $f(x, y) \simeq_{\mathcal{B}} y+x$, germe estável, portanto temos o par estável $(d x, d(y+x))$. 
Se o contato da folha de $\beta$ na origem com o bordo é $k$, ela pode ser escrita da forma $y=a x^{k}+\ldots$, ou equivalentemente $f(x, y)=y+x^{k}\left(1+g_{1}(x)\right)+y \phi(x, y)$. Aplicando mudanças de coordenadas pertencentes a $\mathcal{B}$ temos que o germe $f$ pode ser escrito na forma $f(x, y)=y \pm x^{k}$ (germe $\mathcal{B}$-finitamente determinado, ver Proposição 3.1.4). De onde obtemos o par $\left(d y, d\left(y \pm x^{k}\right)\right.$ ). Além disso, segue da mesma proposição que um desdobramento versal é dado como no enunciado.

Sendo assim, se a folha de $\beta$ na origem é transversal ao bordo, temos o par equivalente a $(d x, d(y+x))$. Se a folha de $\beta$ em zero têm contato $k \geq 2$ com o bordo temos o par equivalente a $\left(d x, d\left(y \pm x^{k}\right)\right)$.

Por outro lado se as folhas de $\alpha$ e $\beta$ na origem têm contato 2 entre si, temos

$$
j^{2} f(x, y)=x \pm y^{2}
$$

então $f(x, y)=x \pm y^{2}+g(x, y)$. Logo o discriminante é dado por $\delta(x, y)= \pm 2 y+g_{y}(x, y)$. Mas se o contato entre o discriminante e o bordo é $k, \delta= \pm 2 y+x^{k}\left(1+g_{1}(x, y)\right)$. Então

$$
f(x, y)=x \pm y^{2}+x^{k} y\left(1+g_{1}(x, y)\right) \simeq_{\mathcal{B}} x \pm y^{2}+x^{k} y
$$

(germe $\mathcal{B}$-finitamente determinado).

Observe que para $k=2 s-1$, os germes $x+x^{2 s-1} y-y^{2}$ e $x+x^{2 s-1} y+y^{2}$ são $\mathcal{B}$-equivalentes, basta considerar o difeomorfismo $h(x, y)=(-x, y)$ e multiplicar $f$ por -1 .

Sendo assim temos dois pares distintos:

(i) $\left(d x, d\left(x \pm y^{2}+x^{2 s} y\right)\right)$

(ii) $\left(d x, d\left(x-y^{2}+x^{2 s-1} y\right)\right)$.

Um desdobramento versal para esses pares segue do desdobramento versal do germe $f$, veja Proposição 3.1.8.

Se o contato for $\geq 3$ temos a Proposição 3.1 .9 e a Proposição 3.1 .10 que asseguram que não existe germe $\mathcal{B}$-finitamente determinado.

A partir da classificação acima podemos enunciar o seguinte corolário:

Corolário 3.1.14 As configurações genéricas das linhas de curvatura e das linhas assintóticas numa vizinhança de um ponto $p$ do bordo (fora da curva parabólica, no caso das linhas assintóticas) são dadas na Figura 3.1.

\subsubsection{Estudo geométrico}

Como no capítulo 2, todas as informaçōes sobre o par $(d x, d f)$ são dadas pelas $\mathcal{B}$-singularidades de $f$, em particular todas as deformações deste par podem ser obtidas a partir das deformações versais de $f$.

Neste caso temos que o conjunto de bifurcação é dado por duas componentes: 
(1) O conjunto dos valores $u$ para os quais $\alpha$ e $\beta$ são transversais e o contato entre as folhas de $\beta$ e o bordo é maior ou igual a 3 , chamamos este conjunto de componente de bordo.

(2) O conjunto dos valores $u$ tal que o contato entre as folhas de $\alpha$ e $\beta$ é 2, sendo ambas transversais ao bordo e o contato entre o discriminante e o bordo é maior ou igual a 2 .

Sendo assim temos que:

$$
B i f(F)=\left\{u: f_{x}^{u}=f_{x x}^{u}=y=0\right\} \cup\left\{u: f_{y}^{u}=f_{y x}^{u}=0=y\right\}
$$

Vamos estudar o conjunto de bifurcação de cada par de germe classificado de $\mathcal{B}_{e}$-codimensão menor ou igual a 2 :

(i) Dado o par $(d x, d(y+x)$ ) (folhas de $\beta$ transversal ao bordo), $\delta=1$ (folhas transversais entre si), logo $B i f(f)=\emptyset$ (veja Figura 3.1, no canto superior esquerdo).

(ii) Dada a família $\left(d x, d\left(y \pm x^{k}+u_{k-2} x^{k-2}+\ldots+u_{1} x\right)\right)$, com $k \geq 2$ temos $\delta(x, y)=1$, logo o conjunto de bifurcação tem apenas a componente de bordo. Para $k=20$ par é estável, então o conjunto de bifurcação é vazio. Observe ainda que as folhas de $\beta$ têm contato $k$ com o bordo, então o conjunto de bifurcação do germe $f$ coincide com o conjunto de bifurcação das $\mathcal{R}$-singularidades $A_{k \geq 2}$ de $f(x, 0)$. Veja na Figura 3.2 as deformações genéricas desse par para $k=3$ e na Figura 3.3 as deformações versais do par de germes $\left(d x, d\left(y \pm x^{4}\right)\right)$.

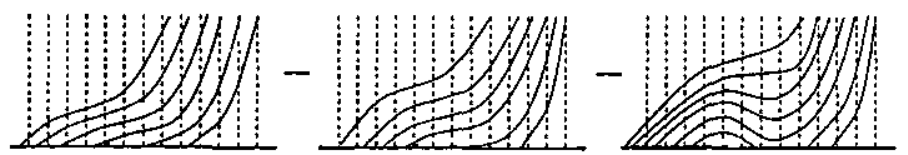

Figura 3.2: Deformações genéricas do par $\left(d x, d\left(y-x^{3}\right)\right)$
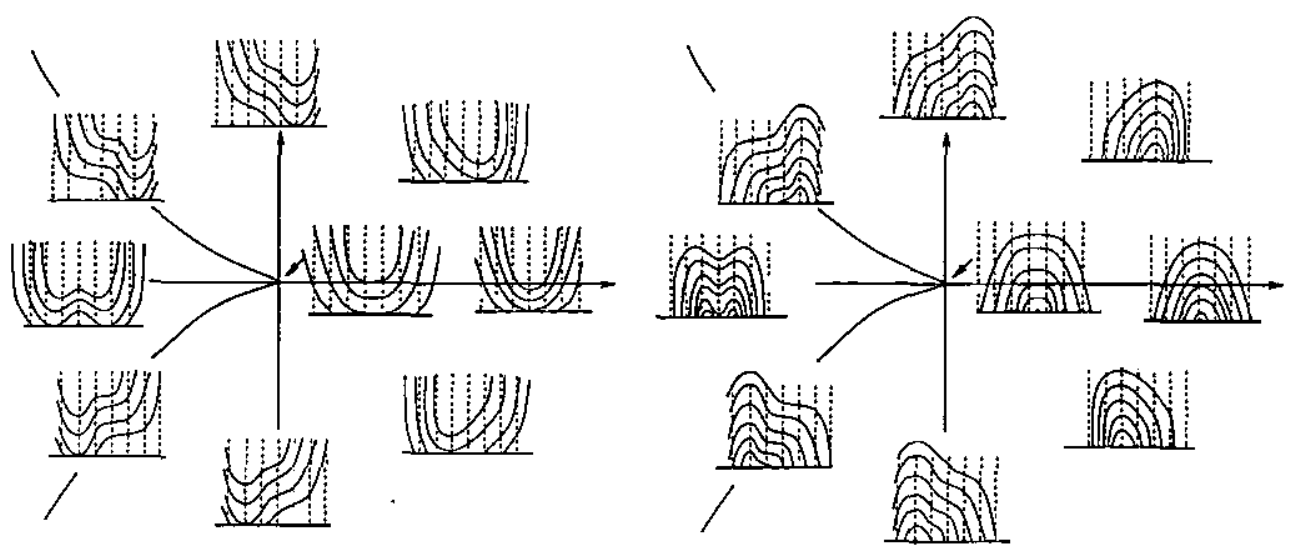

Figura 3.3: Deformações genéricas dos pares $\left(d x, d\left(y+x^{4}\right)\right)$ e $\left(d x, d\left(y-x^{4}\right)\right)$

Na Figura 3.3 observamos que as deformações apresentam duas singularidades no mesmo nível. Para verificar esse fato, basta observar que se $p_{i}$ é um ponto singular de $f$ no mesmo 
nível então $p_{i}=\left(p_{i}^{\prime}, 0\right)$, com $p_{i}^{\prime}$ raíz dupla de $\pm x^{4}+u x^{2}+v x+w=0$, para algum $w \in \mathbb{R}$ e $i=1,2$. Agora proceda exatamente como no capítulo 3 , no estudo geométrico do par $\left(d x, d\left(x \pm y^{4}\right)\right)$ e obterá $v=0$ e $u \in \mathbb{R}, 0$.

(iii) Dado o par $\left(d x, d\left(x+x y+y^{2}\right)\right)$, temos $\delta=2 y+x$, ou seja, o discriminante é regular e é transversal ao bordo, logo $B i f(f)=\emptyset$. Ver Figura 3.1 (canto inferior direito).

(iv) Dada a família $\left(d x, d\left(x+x^{k} y \pm y^{2}+u_{k-2} x^{k-2} y+\ldots+u_{1} x y+u_{0} y\right)\right)$, para $k \geq 2$, temos $f_{y}^{u}(x, y)=-2 y+x^{k}+u_{k-2} x^{k-2}+\ldots+u_{2} x^{2}+u_{1} x+u_{0}, y \geq 0$, ou seja, o discriminante é regular para todos os valores de $k \geq 2$.

Observe que $f_{y}^{0}(x, 0)=x^{k}$, ou seja, $f_{y}^{0}(x, 0)$ possui uma singularidade do tipo $A_{k-1}, k \geq 2$, sendo $f_{y}^{u}(x, 0)$ uma deformação versal de tal singularidade.

Para $k=2$ temos $f_{y}^{u}(x, y)= \pm 2 y+x^{2}+u_{0}$, logo o contato entre as folhas de $d x$ e $d f$ é dois, como também o contato entre o discriminante e o bordo, portanto temos $B i f(f)=$ $\left\{u: f_{y}^{u}(x, 0)=f_{x y}^{u}(x, 0)=0\right\}=\{0\}$. As deformações genéricas do par de 1-formas com $k=2$ são dadas pela Figura 3.4. Na parte superior temos as deformaçōes genéricas do caso positivo(+) e embaixo, do caso negativo(-).

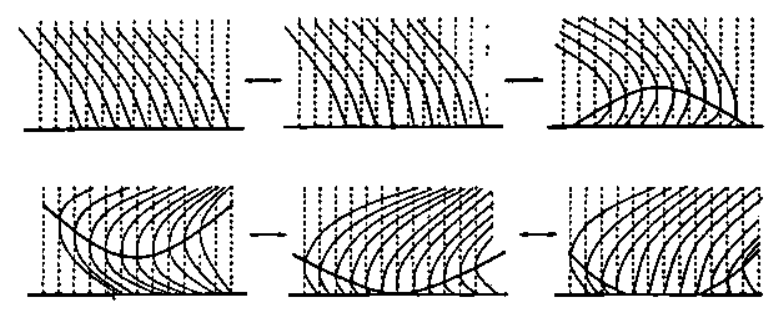

Figura 3.4: Deformáções genéricas de $\left(d x, d\left(x+x^{2} y+y^{2}\right)\right)$ e $\left(d x, d\left(x+x^{2} y-y^{2}\right)\right)$

Para o caso $k=3$, temos $\delta^{u}=f_{y}^{u}=x^{3}+u_{1} x+u_{0} \pm 2 y=0$, então $\delta^{0}$ tem contato 3 com $y=0$ e o conjunto de bifurcação é dado pelos valores de $u$ que satisfazem $f_{y}=f_{x y}=0$. Desde que

$$
\begin{aligned}
f_{y}(x, 0) & =x^{3}+u_{1} x+u_{0}=0 \\
f_{x y}(x, 0) & =3 x^{2}+u_{1}=0
\end{aligned}
$$

ou seja, $\left(u_{0}, u_{1}\right)=\left(2 x^{3},-3 x^{2}\right)$, uma cúspide. As deformações genéricas desse par são dadas pela Figura 3.5

\subsubsection{Invariantes}

A classificação dos pares de germes de 1-formas $(d x, d f)$ em variedades com bordo regular sugere alguns invariantes. Porém notenios que fora do bordo as folhas são transversais, ou possuem contato 2 entre si, logo os pontos que merecem maịor atenção aparecem sobre o bordo. 


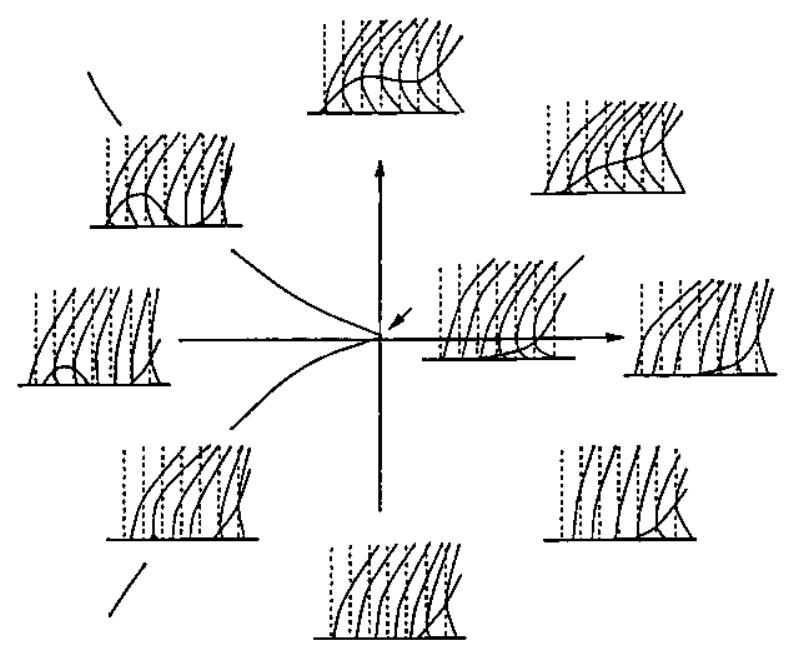

Figura 3.5: Deformaçōes genéricas do par $\left(d x, d\left(x+x^{3} y-y^{2}\right)\right)$

Em deformaçōes versais dos pares classificados observamos que os pontos do bordo que têm tangência de ordem 2 com uma folha de $d f$ (singularidade do tipo $b_{2}^{ \pm}$) ou os pontos do bordo que pertencem ao discriminante (singularidade do tipo $d_{1}$ ) são pontos isolados, portanto esperamos poder enumerá-los em deformações genéricas de pares $(d x, d f)$ com $f$ germe $\mathcal{B}$-finitamente determinado. Observando esse comportamento associamos dois invariantes. O primeiro deles é o número máximo de pontos soluções da equação $f_{x}(x, 0)=0$ (tangência maior ou igual a dois entre a folha da 1-forma $d f$ e o bordo, singularidade $b_{2}^{ \pm}$) e o segundo, o número máximo de soluções da equação $f_{y}(x, 0)=0$ (pontos de intersecção entre o discriminante e o bordo, singularidade $d_{1}$ ). Estes números são denotados por

$$
\sharp b_{2}^{ \pm}(f)=\operatorname{dim}_{\mathbb{R}} \frac{\mathcal{E}_{1}}{\left.<f_{x}(x, 0)\right\rangle}
$$

e

$$
\sharp d_{1}(f)=\operatorname{dim}_{\mathbb{R}} \frac{\mathcal{E}_{1}}{<f_{y}(x, 0)>}
$$

respectivamente.

Novamente abusamos da notação escrevendo $\sharp b_{2}^{ \pm}(f)$ para $\sharp b_{2}^{ \pm}(d y, d f)$, analogamente para $\sharp d_{1}^{ \pm}(f)$.

$\mathrm{Na}$ próxima proposição verificamos que esses são de fato invariantes.

Proposição 3.1.15 Dados os pares de germes $(d x, d f)$ e $(d x, d g)$ com $f \simeq_{\mathcal{B}} g$, temos $\sharp b_{2}^{ \pm}(f)=$ $\sharp b_{2}^{ \pm}(g)$ e $\sharp d_{1}(f)=\sharp d_{1}(g)$.

Demonstração: A demonstração segue os passos da demonstração feita nos capítulos 2 e 3 para $\sharp 3_{1}$ e $\sharp 2_{1}$. 
Além disso, $\sharp b_{2}^{ \pm}$é um invariante topológico desde que coincide com o número de Milnor do germe $\left.f\right|_{\text {bordo }}$ complexificado. Quanto ao invariante $\sharp d_{1}$, sabemos que este é suave como conseqüência da Proposição 3.1.15. Porém este nāo é topológico, como mostra o exemplo abaixo.

Exemplo 3.3 Sejam $f, g: \mathbb{R}^{2}, 0 \rightarrow \mathbb{R}, 0$ definidos por $f(x, y)=x+y^{2}$ e $g(x, y)=x-2 x^{2} y+y^{2}$. Observamos que $f$ e g säo germes topologicamente equivalentes, através do homeomorfismo que preserva as folhas da 1-forma $\alpha=d x$, dado por $h(x, y)=\left(x\left(1-x^{3}\right), y-x^{2}\right)$.

No entanto, $\sharp d_{1}(f)=\infty$ enquanto que $\sharp d_{1}(g)=2$.

Como ocorre com os invariantes anteriormente estudados, o número máximo de pontos $b_{2}^{ \pm}$e $d_{1}$ é atingido por uma deformação real do par $(d x, d f)$ tal que $f$ é germe $\mathcal{B}$-finitamente determinado.

Lema 3.1.16 Seja $(d x, d f)$ um par de germes de 1 -formas com $f$ germe $\mathcal{B}$-finitamente determinado. Então $\sharp b_{2}^{ \pm}(f)=k-1 e \sharp d_{1}(f)=0$ ou $\sharp b_{2}^{ \pm}(f)=0$ e $\sharp d_{1}(f)=k$. Além disso existe uma deformaçāo real de $f$ tal que o número máximo de pontos $b_{2}^{ \pm}$e $d_{1}$ são observados.

Demonstração: Da classificação dos germes $\mathcal{B}$ determinados temos os pares $\left(d x, d\left(y \pm x^{k}\right)\right)$ e $\left(d x, d\left(x \pm x^{k} y+y^{2}\right)\right)$.

Dado o par de germes $\left(d x, d\left(y \pm x^{k}\right), k \geq 2\right.$ temos $f(x, 0)=k x^{k-1}$ então $\frac{\mathcal{E}_{1}}{\left\langle x^{k-1}\right\rangle}=\mathbb{R}$. $\left\{1, x, \ldots, x^{k-2}\right\}$ e $\sharp b_{2}^{ \pm}(f)=k-1$. Além disso uma deformação genérica desse par é dada por $\left(d x, d\left(y \pm x^{k}+u_{k-2} x^{k-2}+\ldots+u_{1} x\right)\right)$ donde temos $f(x, 0)=0$ se e somente se, $k x^{k-1}+(k-$ 2) $u_{k-2} x^{k-1}+\ldots+u_{1}=0$. Escolhendo $u_{k-2}, \ldots, u_{1}$ de modo conveniente o polinômio anterior terá $k-1$ zeros, portanto o número máximo de pontos $b_{2}^{ \pm}$é atingido por deformação real de $f$.

Dado o par $\left(d x, d\left(x \pm x^{k} y+y^{2}\right)\right)$, temos um par transversal ao bordo e com contato 2 entre si, sendo que $\frac{\mathcal{E}_{1}}{\left\langle f_{y}(x, 0)\right\rangle}=\mathbb{R} \cdot\left\{1, x, \ldots, x^{k-1}\right\}$, ou seja, $\sharp d_{1}(f)=k$. Analogamente temos que existe deformação real do par de germes de modo que o número máximo de pontos $d_{1}$ é observado, pois uma deformaçāo genérica deste par é dada por $\left(d x, d\left(x \pm x^{k} y+y^{2}+u_{k-2} x^{k-2} y+\ldots+u_{1} x y+u_{0} y\right)\right)$.

\section{$3.2 \mathcal{B}_{\alpha}$-Classificação de germes $\mathbb{R}^{2}, 0 \rightarrow \mathbb{R}, 0$}

Estudamos agora pares de folheações definidas por 1-formas diferenciais $(\alpha, \beta)$ sobre uma variedade de dimensão 2 com bordo regular supondo que o bordo desta variedade é uma folha da 1-forma $\alpha$.

Considerando cartas locais podemos supor que esta variedade é parametrizada por $\{(x, y)$ : $y \geq 0\}$ e $y=0$ é o bordo da variedade. Entāo se $\alpha$ tem o bordo como folha e é germe de 1-forma regular, podemos supor que $\alpha=d y$. Vamos nos restringir ao caso em que $\beta=d f$ e estudar pares de germes de 1-formas (dy, df). 
Considerando mudanças de coordenadas que preservem as folhas da 1-forma $d y$ e o bordo $y=0$, a classificação do germe $f$ nos dará a classificação dos pares de germes de 1-formas $(d \dot{y}, d f)$. Então as mudanças na fonte devem ter a forma

$$
h(x, y)=\left(h_{1}(x, y), y h_{2}(y)\right)
$$

$\operatorname{com} \frac{\partial h_{1}}{\partial x}(0,0) \cdot h_{2}(0) \geq 0$ sem restrições para mudança na meta.

Estas mudanças formam um subgrupo do grupo $\mathcal{A}$, que denotamos por $\mathcal{B}_{\alpha}$. Além disso, o espaço tangente a $\mathcal{B}_{\alpha}$ é dado por

$$
L \mathcal{B}_{\alpha} \cdot f=\mathcal{M}_{2}\left\{f_{x}\right\}+\mathcal{O}_{y}\left\{y f_{y}\right\}+L \mathcal{L} \cdot f
$$

Logo os espaços $L \mathcal{A}^{*} \cdot f$ e $L B_{\alpha} \cdot f$ coincidem, a diferença ocorre apenas nos espaços tangentes estendidos. Conseqüentemente todo germe $\mathcal{A}^{*}$-finitamente determinado é também $\mathcal{B}_{\alpha^{-}}$ finitamente determinado, apenas as codimensōes podem ser diferentes.

Além disso estamos considerando os germes definidos em $\left\{(x, y) \in \mathbb{R}^{2}: y \geq 0\right\}$, logo os germes acima apresentarāo também geometrias diferentes.

\subsubsection{Caso regular}

Da classificação obtida no capítulo 2 pelo grupo $\mathcal{A}^{*}$, para pares regulares, temos o seguinte teorema:

Teorema 3.2.1 Seja $(\alpha, \beta)$ um par de germes de 1-formas regulares definidas numa variedade bidimensional com bordo regular, tal que o bordo é folha da 1-forma $\alpha$. Então, de acordo com o contato de $\beta$ na origem com o bordo, temos os seguintes casos:

(i) Se $\beta$ é 1-forma transversal ao bordo então $(\alpha, \beta)$ é suavemente equivalente o par $(d y, d x)$ o qual é estável.

(ii) Se o contato de $\beta$ na origem com o bordo é 2 então $(\alpha, \beta)$ é suavemente equivalente a $\left(d y, d\left(y \pm x^{2}\right)\right)$, o qual é estável.

(iii)Se o contato de $\beta$ na origem com o bordo é 3 , entāo $(\alpha, \beta)$ é suavemente equivalente a um dos seguintes pares:

- $\left(d y, d\left(y \pm x y^{2 p+1}+x^{3}\right)\right)$, com $p \geq 0$, com desdobramento versal $\left(d y, d\left(y+x\left(y^{2 p+1}+\right.\right.\right.$ $\left.\left.\left.u_{2 p} y^{2 p}+\ldots+u_{0}\right)+x^{3}\right)\right)$

- $\left(d y, d\left(y \pm x y^{2}+x^{3}\right)\right)$, com desdobramento versal $\left(d y, d\left(y+x\left( \pm y^{2}+u_{1} y+u_{0}\right)+x^{3}\right)\right)$

- $\left(d y, d\left(y+x\left( \pm y^{2 p}+\lambda y^{5 p-1}\right)+x^{3}\right)\right)$, com $p>1$ e desdobramento versal $(d y, d(y+$ $\left.\left.x\left( \pm y^{2 p}+(\lambda+\mu) y^{5 p-1}+u_{2 p-1} y^{2 p-1}+\ldots+u_{0}\right)+x^{3}\right)\right)$ 
(iv) Se o contato de $\beta$ na origem com o bordo é $\geq 4$, não existem modelos suaves de codimensão finita para o par de 1 -formas $(\alpha, \beta)$.

Demonstração: Nas condiçōes do teorema podemos supor que $\alpha=d y$ e $\beta=d f$, com $f: \mathbb{R}^{2}, 0 \rightarrow \mathbb{R}, 0$. Então a folha de $d f$ tem contato $k$ com o bordo na origem significa que $d y$ e $d f$ tem contato $k$ na origem, portanto a partir da $\mathcal{A}^{*}$-classificação dos pares $(d y, d f)$, (que coincide com a $\mathcal{B}_{\alpha}$-classificação) temos (dy,df) suavemente equivalente a um dos pares em (i), (ii), (iii) se o contato for $\leq 3$. Caso o contato seja $\geq 4$ vimos na $\mathcal{A}^{*}$-classificação, que não existem pares de codimensão finita, logo temos (iv).

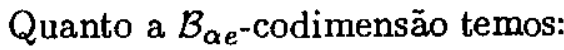

- (i) Para $f(x, y)=x$ temos $f_{x}=1$ e $y f_{y}=0$ então $L \mathcal{B}_{\alpha e} \cdot f=\mathcal{O}_{2}$, isto é, este é um germe $\mathcal{B}_{\alpha}$-estável.

- (ii) Para $f(x, y)=y \pm x^{2}$ temos $f_{x}= \pm 2 x$ e $y f_{y}=y$ logo este também é um germe $\mathcal{B}_{\alpha}$-estável. No entanto neste caso os germes $f(x, y)=y-x^{2}$ e $g(x, y)=y+x^{2}$ não são $\mathcal{B}_{\alpha}$-equivalentes, basta considerar as curvas de nível $f^{-1}(0)$ e $g^{-1}(0) . g^{-1}(0)$ é um ponto, enquanto que $f^{-1}(0)$ é uma curva suave.

- (iii) Para $f(x, y)=y \pm x y^{2 p+1}+x^{3}, \operatorname{com} p \geq 0$ temos $\mathcal{E}_{2} / L \mathcal{B}_{\alpha e} \cdot f=\mathbb{R} \cdot\left\{x, x y, \ldots, x y^{2 p}\right\}$,

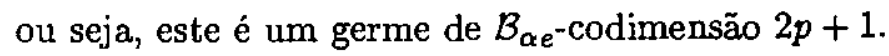

- (iv) Se $f(x, y)=y \pm x y^{2}+x^{3}$ temos $f_{x}= \pm y^{2}+3 x^{2}$ e $y f_{y}=y \pm 2 x y^{2}$, então $\mathcal{E}_{2} / L \mathcal{B}_{\alpha e} \cdot f=$ $\mathbb{R} \cdot\{x, x y\}$ e a codimensão é dois.

- (v) Se $f(x, y)=y+x\left( \pm y^{2 p}+\lambda y^{5 p-1}\right)+x^{3}$, com $p>1$ temos um par de $\mathcal{B}_{\alpha e^{-c o d i m e n s a ̃ o}}$ $2 p+1$ e estrato de codimensão $2 p$, afinal $\mathcal{E}_{2} / L \mathcal{B}_{\alpha e} \cdot f=\mathbb{R} \cdot\left\{x, x y, \ldots, x y^{2 p-1}, x y^{5 p-1}\right\}$.

\subsubsection{Estudo geométrico}

Seja $(d y, d f)$ um par de 1-formas diferenciais com $f \mathcal{B}_{\alpha}$-finitamente determinada, como nos casos anteriores, todas as informaçōes sobre o par de 1-formas são dadas pelas $\mathcal{B}_{\alpha}$-singularidades do germe $f$. Vamos então estudar as deformações de $f$ através de uma deformação versal deste germe e do conjunto de bifurcação.

O conjunto de bifurcação é dado pelos valores de $u$ tais que $f_{u}$ não é estável. Temos duas possibilidades:

- o ponto pertence ao bordo: o contato de $d f_{u}$ na origem com o bordo é $\geq 3$. 
- o ponto não pertence ao bordo: então o ponto pertence ao discriminante que é uma curva singular e o contato entre uma folha de $d f_{u}$ e $d y$ é 3. (compare com o capítulo 1).

Então podemos escrever

$$
B i f(F)=\left\{u ; y=f_{x}^{u}=f_{x x}^{u}=0\right\} \cup\left\{u ; f_{x}^{u}=f_{x x}^{u}=f_{x y}^{u}=0\right\}
$$

Os pares em descritos nos items (i) e (ii) da Proposição 3.2.1 sāo os pares estáveis, logo nestes casos o conjunto de bifurcação é vazio. Veja a Figura 3.6.
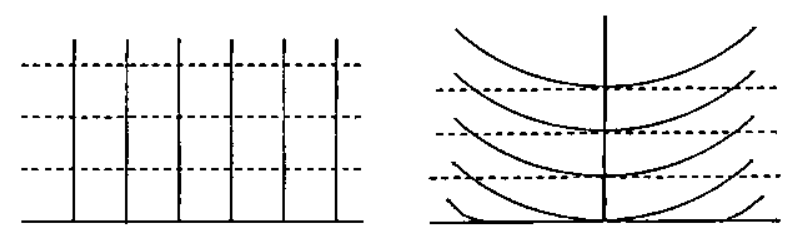

Figura 3.6: Pares estáveis em variedade bidimensional com bordo

Quanto aos demais pares temos:

- Dada a família $\left(d y, d\left(y \pm x y+x^{3}+u_{0} x\right)\right)$, temos que o contato entre $d f$ na origem e o bordo é 3 , além disso $\delta$ é regular, então o conjunto de bifurcação é dado pela primeira componente. Como

$$
\begin{aligned}
f_{x}^{u} & = \pm y+3 x^{2}+u_{0}=0 \\
f_{x x}^{u} & =6 x=0
\end{aligned}
$$

e para $y=0$ temos $u_{0}=0$, o conjunto de bifurcação é um ponto isolado, a origem. Na Figura 3.7 temos as deformações genéricas dessa família. Note que $f^{0}(x, 0)$ tem singularidade $B_{2}$ (para singularidades $B_{k}$, singularidades de funções em variedades com bordo, veja [3]).

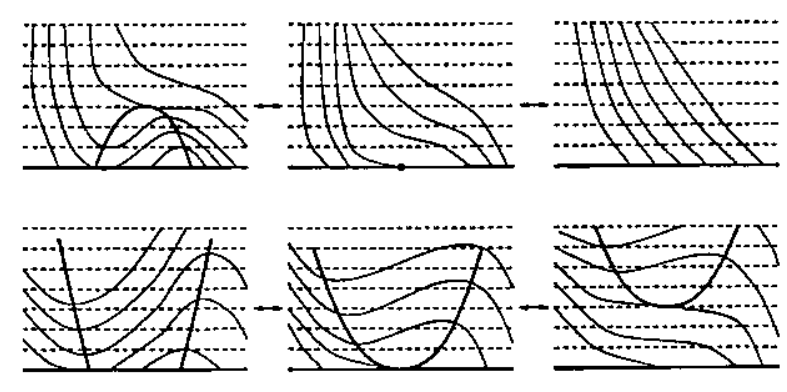

Figura 3.7: Deformaçōes genéricas do par $\left(d y, d\left(y+x y+x^{3}\right)\right)$

- Dada a família $\left(d y, d\left(y \pm x y^{2}+x^{3}+u_{1} x y+u_{0} x\right)\right)$, temos contato 3 entre a 1 -forma $d f$ na origem e o bordo e $\delta$ é uma curva singular então o conjunto de bifurcação é tem duas componentes. Observe ainda que

$$
\begin{aligned}
f_{x}^{u} & = \pm y^{2}+3 x^{2}+u_{1} y+u_{0} & f_{y}^{u} & =1+2 x y+u_{1} x \\
f_{x x}^{u} & =6 x & f_{x y}^{u} & = \pm 2 y+u_{1},
\end{aligned}
$$


então da primeira componente temos $f_{x}^{u}(x, 0)=3 x^{2}+u_{0}=0$ e $f_{y}^{u}(x, 0)=1+u_{1} x=$ 0 , o que significa que em uma vizinhança suficientemente pequena de zero, temos que $u_{0}=0$ e $u_{1} \neq 0$. Enquanto que da segunda componente do conjunto de bifurcação temos $u_{1}=\mp 2 y, u_{1}= \pm y^{2}$ com $y \geq 0$. Portanto

$$
B i f(F)=\left\{\left(u_{1}, 0\right)\right\} \cup\left\{\left(u_{1}, u_{0}\right): u_{1}=\mp 2 y, u_{0}= \pm y^{2}, y \geq 0\right\}
$$

$\mathrm{Na}$ Figura 3.8 exibimos as deformações das duas famf́lias acima estudadas. Observe que o discriminante é formado pelo eixo $O_{x}$ e por um ramo de parábola apenas. $\mathrm{O}$ ramo oposto não pertence ao discriminante devido a restriçäo imposta pelo bordo $(y \geq 0)$.
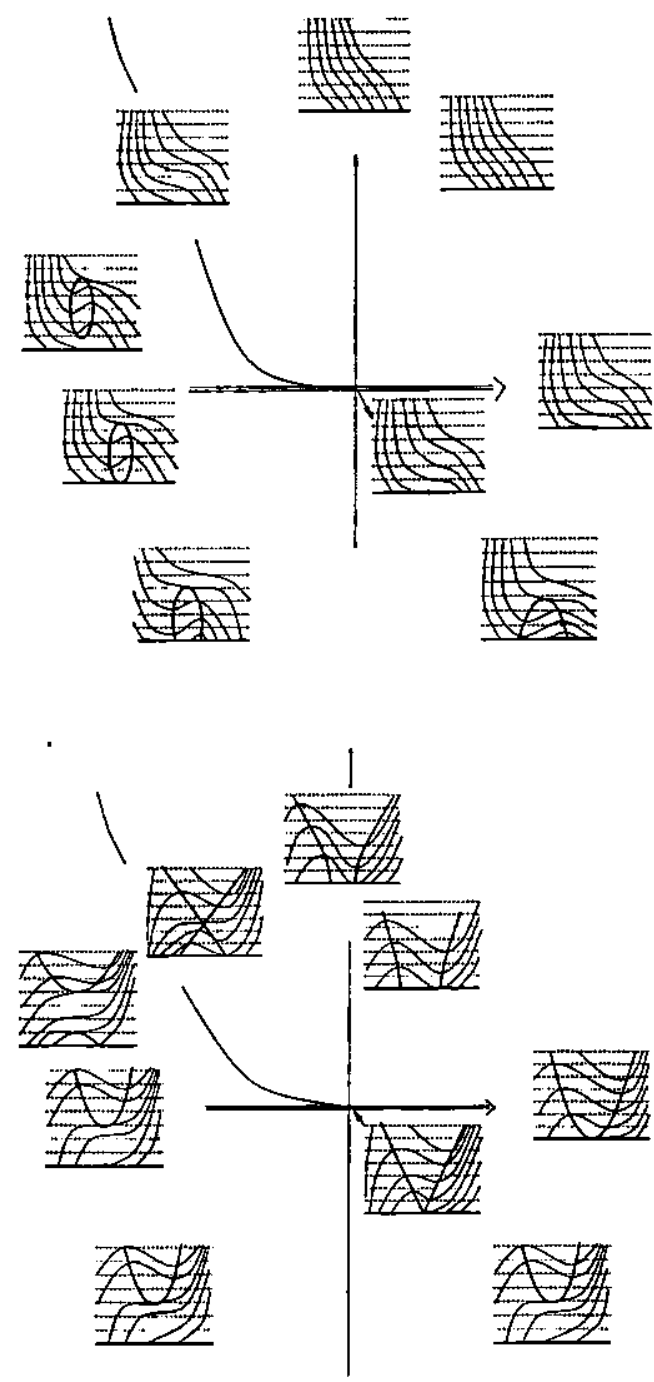

Figura 3.8: Deformaçōes genéricas dos pares $\left(d y, d\left(y+x y^{2}+x^{3}\right)\right)$ e $\left(d y, d\left(y-x y^{2}+x^{3}\right)\right)$

Observaçöes 3.2.2 1. Em geral, dada a familia $\left(d y, d\left(y+x\left( \pm y^{2 p-1}+u_{2 p} y^{2 p}+\ldots+u_{1} y+\right.\right.\right.$ $\left.\left.u_{0}\right)+x^{3}\right)$ ) temos $\delta^{u}= \pm y^{2 p-1}+u_{2 p} y^{2 p}+\ldots+u_{1} y+u_{0}+3 x^{2}$, ou seja, o discriminante $\delta^{0}$ 
tem singularidade $B_{2 p-1}$ e a familia $\delta^{u}$ é um desdobramento versal de tal singularidade. $O$ mesmo acontece a familia $\left(d y, d\left(y+x\left( \pm y^{2 p}+(\lambda+\mu) y^{5 p-1}+u_{2 p-1} y^{2 p-1}+\ldots+u_{0}\right)+x^{3}\right)\right)$, o discriminante $\delta^{0}$ tem singularidade $B_{2 p}$ e $\delta^{u}$ é o desdobramento de tal singularidade. Em ambos os casos o conjunto de bifurcação de $F$ é igual ao $\mathcal{R}_{\alpha}$-conjunto de bifurcação da singularidade $B_{k}$ do discriminante.

2. Como no plano, o módulo não altera a configuração do par de 1-formas, mas não pode ser eliminado por mudanças no grupo $\mathcal{B}_{\alpha}$. Porém de maneira semelhante aos Teoremas 1.7.1 e 1.7.2 obtemos uma equivalência topológica entre os pares $\left(d y, d\left(y+x\left(y^{2 p}+\lambda y^{5 p-1}\right)+x^{3}\right)\right) e$ $(d y, d x)$ e, o par $\left(d y, d\left(y+x\left(-y^{2 p}+\lambda y^{5 p-1}\right)+x^{3}\right)\right) e\left(d y, d\left(y-x y^{2}+x^{3}\right)\right)$ restritos a $y \geq 0$, em variedades com bordo regular.

\subsubsection{Caso singular}

Quando estudamos pares de 1-formas regular/singular, não podemos assegurar que a 1-forma $\beta$ é exata. Contudo neste capítulo restringimos nosso estudo aos pares de 1 -formas $(\alpha, \beta)$, onde as folhas de $\beta$ são curvas de nível de um germe de função diferenciável $f$ no plano, isto é, pares da forma $(d y, d f)$ com $j^{1} f(0,0)=0$. A classificação desses pares de germes segue, assim como no caso regular, da $\mathcal{A}^{*}$-classificação do germe $f$. Donde temos o seguinte teorema:

Teorema 3.2.3 Seja $(\alpha, \beta)$ um par de germes de 1-formas diferenciais regular/singular tal que $\beta$ é 1-forma exata. Então os únicos modelos suaves de codimensão finita são dados pelos pares

$$
\left(d y, d\left(x^{2} \pm y^{k}\right)\right)
$$

para algum valor de $k \geq 2$. Além disso, as deformação versais dos pares acima são dadas por $\left(d y, d\left(x^{2} \pm y^{k}+u_{k-1} y^{k-1}+\ldots+u_{1} y\right)\right)$. Em particular, não temos pares de germes estáveis.

Demonstração: Segue da classificação dos pares $(d y, d f)$ em $\mathbb{R}^{2}, 0$ pelo grupo $\mathcal{A}^{*}$, ver Teorema 2.1.6.

Quanto a $\mathcal{B}_{\alpha}$-codimensão temos:

$$
\begin{aligned}
f_{x} & =2 x \\
y f_{y} & =k y^{k},
\end{aligned}
$$

logo segue que $\mathcal{E}_{2} / L \mathcal{B}_{\alpha e} \cdot f=\mathbb{R} \cdot\left\{y, \ldots, y^{k-1}\right\}$, donde temos o desdobramento enunciado.

\subsubsection{Estudo geométrico}

Como nos demais casos a geometria do par $(d y, d f)$ depende do desdobramento versal do germe $f$ no bordo.

Neste caso o conjunto de bifurcação é dado por duas componentes: 
- O conjunto dos valores de $u$ para os quais $f^{u}$ tem uma singularidade no bordo

- O conjunto dos valores de $u$ para os quais $f^{u}$ tem uma singularidade $B_{k \geq 2}$ fora do bordo.

Ou seja,

$$
B i f(F)=\left\{u: f_{x}^{u}=f_{y}^{u}=y=0\right\} \cup\left\{u: f_{x}^{u}=f_{y}^{u}=\left(f_{x y}^{u}\right)^{2}-f_{x x}^{u} f_{y y}^{u}=0\right\}
$$

Dado o germe $\left(d y, d\left(x^{2} \pm y^{2}\right)\right)$ temos sua deformação versal dada por $\left(d y, d\left(x^{2} \pm y^{2}+\right.\right.$ $\left.u_{0} y\right)$ ). Neste caso $f^{u}$ tem singularidade Morse, logo o conjunto de bifurcação é dado por $B i f(F)=\left\{u: f_{x}^{u}=f_{y}^{u}=y=0\right\}$. Desde que $f_{x}^{u}(x, 0)=2 x=0, f_{y}^{u}(x, 0)=u_{0}=0$, 。 conjunto de bifurcação é a origem. Ver a Figura 3.9 para as deformações versais dessa família.

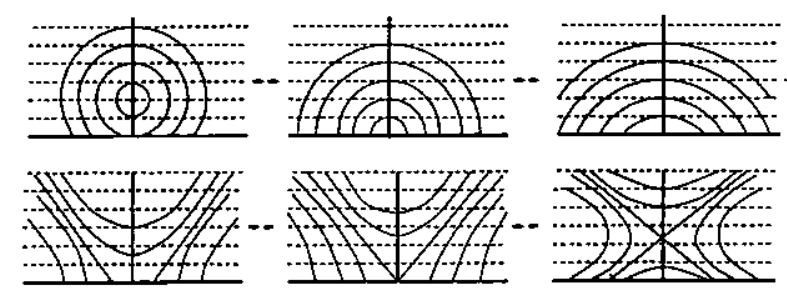

Figura 3.9: Deformações genéricas de $\left(d y, d\left(x^{2} \pm y^{2}+u_{0} y\right)\right)$

Para $k=3$ a família é dada por $\left(d y, d\left(x^{2} \pm y^{3}+u_{0} y^{2}+u_{1} y\right)\right), f^{u}$ tem singularidade $B_{2}$ então o conjunto de bifurcação é formado pelas duas componentes. Desde que

$$
\begin{aligned}
f_{x}^{u} & =2 x \\
\left(f_{x y}^{u}\right)^{2}-f_{x x}^{u} f_{y y}^{u} & =0-2\left( \pm 6 y+2 u_{0}\right)
\end{aligned} \quad f_{y}^{u}= \pm 3 y^{2}+2 u_{0} y+u_{1}
$$

temos $f_{x}(x, 0)=x=0, f_{y}^{u}(x, 0)=u_{1}=0$. Portanto da primeira componente do conjunto de bifurcação temos os pontos $u_{1}=0$ e da segunda componente temos $u_{0}=\mp 3 y, u_{1}= \pm 3 y^{2}, y \geq 0$.

Sendo assim $B i f(F)=\left\{\left(u_{0}, 0\right)\right\} \cup\left\{\left(u_{0}, u_{1}\right): u_{0}=\mp 3 y, u_{1}= \pm 3 y^{2}, y \geq 0\right\}$, que é uma curva regular. Na Figura 3.10 temos as deformações genéricas dessa família. Como no caso regular não vemos o outro ramo da parábola devido a restrição $y \geq 0$.

\subsubsection{Invariantes}

A classificação dos pares de germes de 1-formas em variedades com bordo regular sugere um invariante geométrico, o número de singularidades do tipo $3_{1}$ e $2_{0}$ que aparecem nos pares de 1-formas fora do bordo, afinal a $\mathcal{B}_{\alpha}$-classificação coincide com a $\mathcal{A}^{*}$-classificação fora dos pontos do bordo; e as singularidades do tipo $b_{2}$ que aparecem no bordo.

Como no capítulo 1, vamos interpretar estes invariantes como o número máximo de zeros 

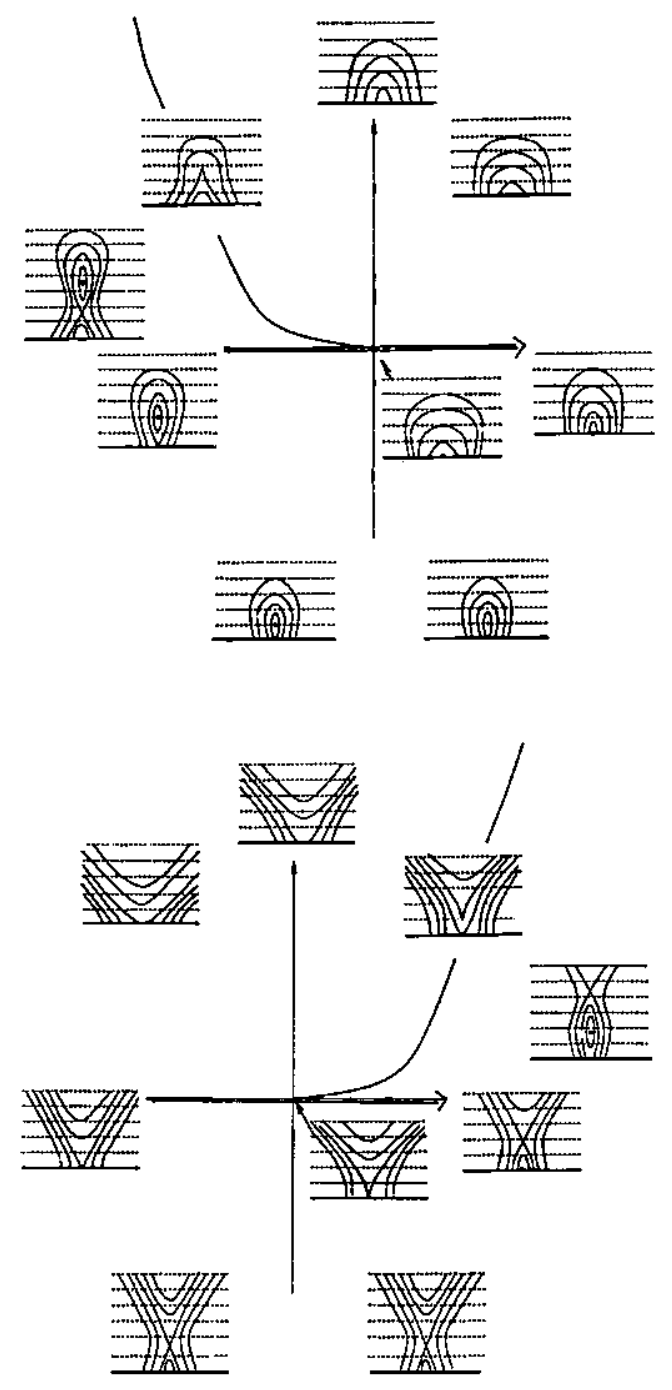

Figura 3.10: Deformaçōes genéricas dos pares $\left(d y, d\left(x^{2}+y^{3}\right)\right)$ e $\left(d y, d\left(x^{2}-y^{3}\right)\right)$

das equações que definem o ideal. Sendo assim definimos:

$$
\begin{aligned}
& \sharp 3_{1}(f)=\operatorname{dim}_{\mathbb{R}} \frac{\mathcal{E}_{2}}{\left\langle f_{x}, f_{x x}\right\rangle} \\
& \sharp 2_{0}(f)=\operatorname{dim}_{\mathbb{R}} \frac{\mathcal{E}_{2}}{\left\langle f_{x}, f_{y}\right\rangle} \\
& \sharp b_{2}(f)=\operatorname{dim}_{\mathbb{R}} \frac{\mathcal{E}_{1}}{\left\langle f_{x}(x, 0)\right\rangle}
\end{aligned}
$$

Como vimos no capítulo $1, \sharp 3_{1}$ e $\sharp 2_{0}$ säo invariantes suaves, mais ainda, $2_{0}$ é um invariante topológico. Além disso, sempre podemos definir uma deformação real do germe $f$ de modo que o número máximo de pontos do tipo $3_{1}$ e $2_{0}$ sejam observados, esse fato pode ser demonstrado seguindo os passos do Lema 1.6.3.

Quanto a $\sharp b_{2}$, vimos no capítulo 2 que este é um invariante topológico, afinal coincide com o número de Milnor do germe $\left.f\right|_{b o r d o}$ complexificado. Neste caso $\sharp b_{2}$ é igual a $I$ se $f$ é um germe $\mathcal{B}_{\alpha}$-finitamente determinado, afinal $f(x, y) \simeq x^{2} \pm y^{k}$, portanto $f_{x}(x, 0)=2 x$. 


\section{Capítulo 4}

\section{Classificação topológica de pares de folheações em variedades de}

\section{dimensão 2}

Neste capítulo apresentamos uma lista de modelos topológicos de pares de germes de 1-formas diferenciais $(\alpha, \beta)$, onde $\alpha, \beta$ são ambos regulares ou têm uma singularidade do tipo sela, nó ou foco. Esta lista cobre todos os fenômenos de codimensão $\leq 1$ exceto aqueles vindos da instabilidade de uma das 1-formas.

Observamos que a folheação com singularidade do tipo nó na origem é topologicamente equivalente a folheação com singularidade do tipo foco [2], mas podemos usar argumentos topológicos para mostrar que o par (regular, nó) não é topologicamente equivalente ao par (regular, foco).

As formas normais deste capítulo foram obtidas usando duas técnicas diferentes. No caso quando ambos os germes de 1-formas são regulares recorremos aos resultados do capítulo 1. Quando um ou ambos os germes de 1-formas são singulares usamos o método do blowing up polar e analisamos o par numa vizinhança da fibra excepcional.

A idéia principal no método do blowing up polar é levantar o par de 1-formas $(\alpha, \beta)$ a um par $\left(\alpha^{\prime}, \beta^{\prime}\right)$ em $S^{1} \times \mathbb{R}$ e produzir modelos via homeomorfismo que se estendam para a fibra excepcional. Assim através do blowing down obtemos um par equivalente na vizinhança da origem. Note que a fibra excepcional é uma folha comum ao par de 1-formas após o blowing up, portanto temos uma situação muito degenerada, de codimensão infinita quando considerada no espaço de todos os pares de 1-formas, mas que não pode ser evitada quando usamos o blowing up.

O estudo feito neste capítulo é topológico mas cobre os pares de folheações da forma regular/singular e singular/singular, onde a folheação singular não é necessariamente definida como curva de nível de função. Estes resultados se aplicam aos sistemas bidimensionais e aos sistemas 
de controle como em [16].

Iniciamos este capítulo com alguns conceitos sobre genericidade e transversalidade. Estes conceitos são usados para definir de maneira mais rigorosa a codimensão dos pares de 1-formas diferenciais no plano nos casos acima mencionados.

\subsection{Genericidade e transversalidade}

O conceito de transversalidade e genericidade é apresentado de maneira clara por Bruce e Giblin em [10] e por Gibson em [18]. Aqui apenas enunciamos os principais resultados que serão necessários no estudo da codimensão de pares de 1-formas no plano. Os principais resultados aqui apresentados foram estabelecidos por $R$. Thom, mas nos referimos ao livro de Gibson [18] para as demonstraçōes.

Definição 4.1.1 Seja $f: \mathbb{R}^{\mathrm{n}} \rightarrow \mathbb{R}^{\mathrm{p}}$ uma aplicação de classe $C^{\infty}$ e $S \subset \mathbb{R}^{\mathrm{p}}$ uma variedade suave. Dizemos que $f$ é transversal a $S$ em $x \in \mathbb{R}^{\mathrm{n}}$ se $f(x) \notin S$ ou para $f(x) \in S$ temos

$$
T_{x} f\left(\mathbb{R}^{\mathfrak{n}}\right)+T_{\mathrm{f}(\mathrm{x})} \mathrm{S}=\mathbb{R}^{\mathrm{p}}
$$

Notação: f↔S. Veja Figura 4.1
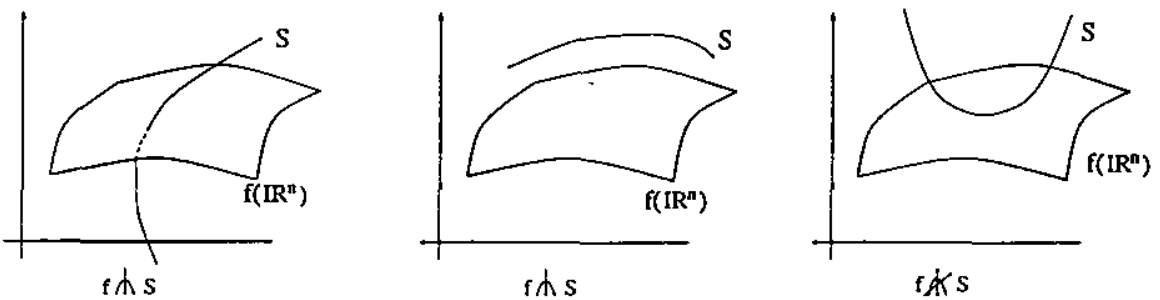

Figura 4.1: Transversalidade de $f$ e $S$

Observe que a equação de transversalidade implica que codim $S \leq n$. Se codim $S>n$ então $f\left(\mathbb{R}^{\mathrm{n}}\right) \cap S=\emptyset$. Além disso, se $S$ é apenas um ponto, dizer que $f$ é transversal a $S$ é equivalente a dizer que $f$ é germe de submersão ou $p \notin f\left(\mathbb{R}^{\mathrm{n}}\right)$.

Proposição 4.1.2 Seja $f: \mathbb{R}^{\mathrm{n}} \rightarrow \mathbb{R}^{\mathrm{p}}$ de classe $C^{\infty}$ e $S \subset \mathbb{R}^{\mathrm{p}}$ subvariedade suave com $f \pitchfork S$. Então $M=f^{-1}(S)$ é uma variedade suave em $\mathbb{R}^{\mathrm{n}}$ de mesma codimensão que $S$ ou entāo $M=\emptyset$.

Lema 4.1.3 Lema da Transversalidade Seja $F: \mathbb{R}^{\mathfrak{n}} \times \mathbb{R}^{\mathrm{q}} \rightarrow \mathbb{R}^{\mathrm{p}}$ uma aplicação de classe $C^{\infty}$ e transversal a subvariedade $S \subset \mathbb{R}^{\mathrm{p}}$. Então existe um conjunto denso de parâmetros $t \in \mathbb{R}^{\mathrm{q}}$ tal que $f_{t} \pitchfork S$, onde $f_{t}(x)=F(t, x)$. 
Ou seja, o lema nos diz que se $t_{0} \in \mathbb{R}^{\mathrm{q}}$ é tal que a transversalidade entre $f_{t_{0}}$ e $S$ não se verifica, existe $t$ suficientemente próximo de $t_{0}$ tal que $f_{t} \pitchfork S$.

Temos o seguinte teorema:

Teorema 4.1.4 $O$ conjunto das aplicações $C^{\infty}\left(\mathbb{R}^{\mathrm{n}}, \mathbb{R}^{\mathrm{p}}\right)$ que são transversais a um variedade $S \subset \mathbb{R}^{\mathrm{p}}$ é denso.

O seguinte teorema é fundamental desde que nos garante que podemos nos restringir ao $k$-jato das aplicações no estudo da transversalidade.

Teorema 4.1.5 Seja $S$ uma subvariedade suave no $J^{k}(n, p)$. O conjunto das aplicaçōes suaves $f: \mathbb{R}^{\mathrm{n}} \rightarrow \mathbb{R}^{\mathrm{p}}$ para os quais $j^{k} f: \mathbb{R}^{\mathrm{n}} \rightarrow \mathrm{J}^{\mathrm{k}}(\mathrm{n}, \mathrm{p})$ é transversal a $S$ é denso em $C^{\infty}\left(\mathbb{R}^{\mathrm{n}}, \mathbb{R}^{\mathrm{p}}\right)$.

Diremos que uma propriedade $P$ é genérica se ela se verifica para um conjunto residual, ou seja, para uma interseç̧ão enumerável de conjuntos abertos e densos de aplicaçōes (para uma definiçāo mais rigorosa veja [10]).

Vejamos agora o que acontece no estudo de pares de folheações no plano definidas por 1formas diferenciais, conjunto que denotamos por $\mathcal{W}$. Vamos identificar $\mathcal{W}$ com o módulo $\mathcal{E}_{2}^{4}$ dos germes de aplicações $\mathbb{R}^{2}, 0 \rightarrow \mathbb{R}^{4}, 0$. Dadas duas 1-formas $\alpha=a(x, y) d x,+b(x, y) d y$ e $\beta=c(x, y) d x+d(x, y) d y$, considere a aplicação $\theta_{0}: \mathbb{R}^{2}, 0 \rightarrow \mathrm{J}^{\mathrm{k}}(2,4)$ dada por $\theta_{0}(x, y)=$ $j^{k}(a, b, c, d)(x, y)$. Consideramos este espaço com a topologia de Whitney [19]. Um par $(\alpha, \beta)$ é dito estável se existe uma vizinhança $V$ de $(\alpha, \beta)$ em $\mathcal{W}$ tal que todos os elementos de $V$ sejam equivalentes a $(\alpha, \beta)$.

Em geral, seja $P$ uma propriedade em $J^{k}(2,4)$ dos pares $(\alpha, \beta)$, por exemplo, $(\alpha, \beta)$ é da forma regular/singular. Suponha que o conjunto $S$ de $k$-jatos de pares que satisfazem a propriedade $P$ é definido por uma aplicação regular $g: J^{k}(2,4) \rightarrow \mathbb{R}^{\mathrm{p}}$, ou seja, $S=g^{-1}(0)$. Dizemos que $(\alpha, \beta) \in S$ tem codimensão $s$ se existir uma família $\theta: \mathbb{R}^{2} \times U \rightarrow J^{\mathrm{k}}(2,4)$, onde $U$ é uma vizinhança da origem em $\mathbb{R}^{\mathrm{s}}$ tal que $\theta(-, 0)=\theta_{0}, \theta \pitchfork S$ e $s$ é a dimensão mínima de $U$ para que $\theta$ satisfaça as duas propriedades acima.

Pela Definição 4.1.1 é claro que $s=\operatorname{codim} S-2$, ou seja, a codimensão de $(\alpha, \beta)$ é o número de condições que definem a propriedade $P$ menos 2 (número de parâmetros $(x, y)$ ).

Por exemplo, suponha que $S$ é conjunto dos pares de 1-formas da forma regular/singular. Defina a aplicação $g: J^{k}(2,4) \rightarrow \mathbb{R}^{2}$ dada por $g\left(j^{k}(a, b, c, d)\right)=\left(c_{0}, d_{0}\right)$, onde $c_{0}$ e $d_{0}$ são os termos constantes de $c$ e $d$ respectivamente. Então $S=g^{-1}(0)$ é uma subvariedade de codimensão 2 , desde que $g$ é regular. Portanto os pares $(\alpha, \beta) \in S$ são de codimensão zero, ou seja, estáveis.

Mas se adicionamos mais uma condição aos pares de 1-formas teremos $S$ de codimensão 3, portanto os pares não serāo mais estáveis, é preciso mais um parâmetro para ter a transversali- 
dade. De modo análogo tratamos os pares singulares que terão codimensão dois, pois precisamos de 4 condições para definir estes pares (todos os termos constantes de $a, b, c$ e $d$ devem ser nulos).

\subsection{Método de blowing up polar}

F. Dumortier [17] usou o método do blowing up polar para o estudo das singularidades de 1-formas diferenciais. A idéia baseia-se em substituir um ponto singular pelo círculo $S^{1}$.

Seja $\alpha$ é uma 1-forma diferencial definida na vizinhança da origem e seja $\phi: S^{1} \times \mathbb{R} \rightarrow \mathbb{R}^{2}$ a aplicação definida por $\phi(\theta, r)=(r \cos \theta, r \operatorname{sen} \theta)$. Existe uma 1-forma $\beta$ em $S^{1} \times \mathbb{R}$, tal que em cada ponto $p \in S^{1} \times \mathbb{R}$, tem-se $\phi_{*}(\beta(p))=\alpha(\phi(p))$.

Denotando por $\mathbb{R}_{+}^{*}$ o conjunto dos número reais positivos, notamos que a restrição de $\phi$ à $S^{1} \times \mathbb{R}_{+}^{*}$ é um difeomorfismo definido no plano exceto a origem.

Sendo assim a 1-forma $\beta$ não é nada mais do que a 1-forma $\alpha$ escrita em coordenadas polares.

Se a 1-forma diferencial tem a propriedade que $j^{k}(\alpha)(0)=0$ e $j^{k+1}(\alpha)(0) \neq 0$, então dividi$\operatorname{mos} \beta$ por $r^{k}$, o que não altera a configuração das folhas da 1-forma. Então daqui para frente, por blowing up, entendemos a forma $\alpha=\left(1 / r^{k}\right) \beta$, onde $k$ é o maior inteiro tal que $j^{k}(\alpha)(0)=0$.

Vejamos alguns exemplos:

Seja a 1-forma linearizável $\alpha=x d y-\lambda y d x$, com singularidade do tipo sela ou nó dependendo do sinal de $\lambda$. Considere a aplicação $\Phi$ definida em $S^{1} \times \mathbb{R}$ por $\Phi(\theta, r)=(r \cos \theta, r \operatorname{sen} \theta)$, assim temos

$$
\begin{aligned}
& x=r \cos \theta \\
& y=r \operatorname{sen} \theta
\end{aligned}
$$

então

$$
\begin{aligned}
& d x=\cos \theta-r \operatorname{sen} \theta d \theta \\
& d y=\operatorname{sen} \theta+r \cos \theta d \theta
\end{aligned}
$$

portanto

$$
\Phi^{*}(\alpha)=r \cos \theta \operatorname{sen} \theta(1-\lambda) d r+r^{2}\left(\cos ^{2} \theta+\lambda \operatorname{sen}^{2} \theta\right) d \theta
$$

e dividindo a equação (4.1) por $r$ obtemos

$$
\Phi^{*}(\alpha)=\cos \theta \operatorname{sen} \theta(1-\lambda) d r+r\left(\cos ^{2} \theta+\lambda \operatorname{sen}^{2} \theta\right) d \theta
$$

Estudando os pontos singulares sobre o círculo $r=0$ encontramos $\theta=0, \frac{\pi}{2}, \pi, \frac{3 \pi}{2}$.

O polinômio característico de $\alpha$ em $(0, \theta)$, para $\theta=0, \pi$ é dado por $t^{2}-(2-\lambda) t+(1-\lambda)$ o que implica que $\delta=\lambda^{2}>0$, ou seja, $(0, \theta)$ serão pontos de sela ou nó, dependendo do sinal de $\lambda$. Para $\theta=\frac{\pi}{2}, \frac{3 \pi}{2}$, o polinômio é dado por $t^{2}-(2 \lambda-1) t-\lambda(1-\lambda)$, assim $\delta=1>0$ e, como no caso acima, a singularidade dependerá do sinal de $\lambda$. 
Assim se $\alpha$ possui singularidade do tipo sela na origem temos que $\lambda<0$, logo $\Phi^{*}(\alpha)$ também possui singularidade do tipo sela nos pontos $\theta=0, \pi, \frac{\pi}{2}, \frac{3 \pi}{2}$ e é regular nos demais pontos do círculo $r=0$.

Para o caso de $\alpha$ possuir singularidade do tipo nó temos que $\lambda>0$, então para $0<\lambda<1$, $\Phi^{*}(\alpha)$ possui singularidade do tipo sela para $\theta=0, \pi$ e do tipo nó, para $\theta=\frac{\pi}{2}, \frac{3 \pi}{2}$ e, é regular nos demais pontos do círculo $r=0$. Para $\lambda>1, \Phi^{*}(\alpha)$ possui singularidade do tipo nó, para $\theta=0, \pi$ e, do tipo sela para $\theta=\frac{\pi}{2}, \frac{3 \pi}{2}$, sendo regular nos demais pontos do círculo $r=0$.

O mesmo pode ser feito para uma forma diferencial com singularidade do tipo foco na origem. Temos que $\alpha=(x-\lambda y) d y-(\lambda x+y) d x$ após a blow up polar como feito no caso sela e nó temos

$$
\Phi^{*}(\alpha)=\lambda d r+r d \theta
$$

Ou seja, $\Phi^{*}(\alpha)$ não possui pontos singulares sobre a curva $r=0$.

\subsection{Resultados auxiliares}

Como mencionamos na introdução deste trabalho, alguns resultados sobre pares de folheações foram demonstrados por Bruce e Fidal [6], Michel [30], Bruce, Fletcher e Tari [7], entre outros. Vamos relembrar as formas normais conhecidas e estabelecer alguns resultados auxiliares que serão usados neste capítulo.

Proposição 4.3.1 ([6]) Sejam $\alpha$ e $\beta$ dois germes de 1-formas regulares com uma folha em comum. Suponha que $\alpha-\beta$ tem um zero não degenerado, então existe um germe de um difeomorfismo levando o par ao modelo $(d y,(1+x) d y-y d x)$.

Michel provou uma versão geral da proposição acima.

Proposiçāo 4.3.2 ([30]) Sejam $\alpha$ e $\beta$ dois germes de 1-formas regulares com uma folha comum. Suponha que o discriminante é dado por $\delta=y^{k+1} \delta_{0}$ com $\delta_{0}(0,0) \neq 0$, em um sistema de coordenadas adequado. Então existe um germe de um difeomorfismo conjugando o par ao modelo $\left(d y, d\left(y e^{y^{k} x}\right)\right)$.

Note que a condição $\alpha-\beta$ tendo um zero não degenerado na Proposição 4.3.1 é equivalente a $\delta=y \delta_{0}$. Também temos o seguinte resultado estabelecido.

Proposição 4.3.3 ([6]) Sejam $\alpha$ e $\beta$ dois germes de 1-formas diferenciais com $\alpha$ regular $e$ $\beta$ com uma singularidade do tipo sela ou nó na origem. Suponha que o par tem uma folha comum pela origem. Então existe um germe de um homeomorfismo levando o par ao modelo $(d y, x d y \pm y d x)$. 
É importante observar que na proposição acima os autovalores do campo de vetores associado à 1-forma singular não precisam satisfazer a condição de não-resonância (ver [14] para a definição).

Nós precisamos de um modelo local para o caso de pares regulares cujo discriminante tem uma singularidade $A_{1}^{-}$, sendo que um ramo do discriminante é uma folha comum ao par de 1-formas e o outro ramo consiste de pontos críticos do Tipo 1 (ver Definição 1.4.1).

Proposição 4.3.4 Sejam $\alpha$ e $\beta$ dois germes de 1-formas regulares. Suponha que o discriminante tem uma singularidade $A_{1}^{-}$onde um dos ramos do discriminante é uma folha comum do par de 1-formas e o outro consiste de pontos do Tipo 1. Então existe um difeomorfismo levando o $\operatorname{par}(\alpha, \beta)$ ao $\operatorname{par}\left(d y, d\left(y\left(1 \pm x^{2}\right)\right)\right)$.
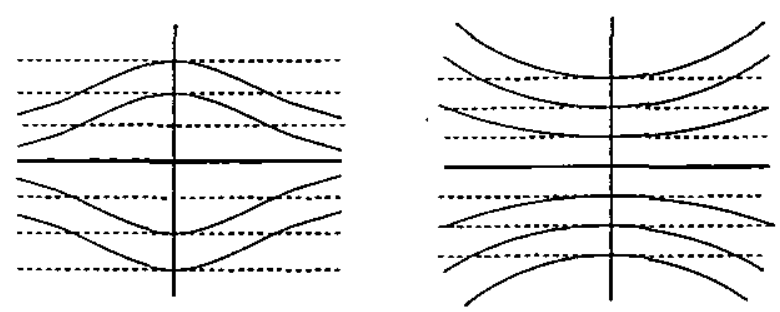

Figura 4.2: $\operatorname{Par}(\alpha, \beta)$ regular/regular onde o discriminante tem singularidade do tipo Morse e uma folha em comum

Demonstração: Pelo Teorema da Retificação podemos assumir que $\alpha=d y$ e que a folheação de $\beta$ é dada pelas curvas de nível de $f(x, y)=y(1+a y+g(x, y))$, para alguma funçāo suave $g$ com 1-jato nulo, e um escalar a. Neste caso o discriminante é dado por $f_{x}(x, y)=y g_{x}(x, y)=0$ e segue das hipóteses que $g_{x}(0,0)=0$ e $g_{x x}(0,0) \neq 0$.

Usando o Splitting Lemma [5], podemos escrever $g(x, y)= \pm x^{2}+k(y)$ através de um difeomorfismo da forma $(\phi(x, y), \psi(y))$, que preserva a folheação $\alpha$. Logo um segundo difeomorfismo preservando as folhas da 1-forma $\alpha$ reduz $f$ à $y\left(1 \pm x^{2}\right)$.

Observamos ainda que os pares $\left(d y, d\left(y\left(1+x^{2}\right)\right)\right)$ e $\left(d y, d\left(y\left(1-x^{2}\right)\right)\right)$ não são topologicamente equivalentes (ver Figura 4.2).

Alguns aspectos do caso onde as duas 1-formas são singulares com separatrizes em comum são estudadas em [7], contudo precisamos do seguinte resultado:

Teorema 4.3.5 Sejam $\alpha$ e $\beta$ dois germes de 1-formas singulares com separatrizes em comum $e$ autovalores (do campos de vetores associados d̀s 1-formas) distintos. Entāo existe um homeomorfismo levando o par $(\alpha, \beta)$ ao par

$$
(x d y \pm y d x, x d y-\mu y d x), \mu \neq 0
$$


Demonstração: A prova segue a construção feita na Proposição 4.3 em [6] para o caso de uma I-forma regular e a outra com singularidade do tipo sela ou nó. A idéia é a seguinte. Por [21], existe um difeomorfismo $C^{1}$ o qual leva a folheação de $\alpha$ às curvas de nível do conjunto definido por $x^{-\lambda} y=$ const. Então podemos fixar $\alpha=x d y-\lambda y d x$.

Seja $j^{1} \beta=x d y-\mu y d x$. Novamente por [21], existe um difeomorfismo local de classe $C^{1}$, $\varphi(x, y)=\left(\varphi_{1}(x, y), \varphi_{2}(x, y)\right)$, com 1-jato igual a identidade, que leva o germe de 1-forma $\beta$ a $j^{1} \beta$. Contudo a folheação de $j^{1} \beta$ é dado pelas curvas de nível do conjunto $x^{-\mu} y=$ const.

Considere a linha $x=\epsilon$ para um $\epsilon$ pequeno e trabalhe no primeiro quadrante. A folha $C$ de $\beta$ pelo ponto $(x, y)$ intercepta a linha $x=\epsilon$ em um ponto $(\epsilon, c)$. Determine $c$ resolvendo a equação $\varphi_{1}^{-1}(x, y) \cdot \varphi_{2}(x, y)=\varphi_{1}^{-1}(\epsilon, c) \cdot \varphi_{2}(\epsilon, c)$. Considere agora o germe de função $h: \mathbb{R}, 0 \rightarrow \mathbb{R}, 0$ definido por $h(c)=\varphi_{1}^{-1}(\epsilon, c) \cdot \varphi_{2}(\epsilon, c)$. Como mostrado em [6], $h$ é de classe $C^{1}$ então a função $g(x, y)=h^{-1}\left(\varphi_{1}^{-1}(x, y) \cdot \varphi_{2}(x, y)\right)$ é de classe $C^{1}$ fora do eixo $O_{y}$ e $g(0, y)=0$.(O valor de $g(x, y)$ é exatamente o escalar $c$ ).

A idéia agora é ir da folha $C$ até a folha de $j^{1} \beta$ andando ao longo das curvas de nível de $x^{-\lambda} y$, veja a Figura 4.3. Esta transformação envia um ponto $(x, y)$ a um ponto $(X, Y)$ cujas coordenadas são obtidas pela solução do sistema $X^{-\lambda} Y=x^{-\lambda} y$ e $X^{-\mu} Y=\epsilon^{-\mu} c=\epsilon^{-\mu} g(x, y)$. Assumindo que $\lambda>\mu$, a solução é dada por

$$
\begin{aligned}
X & =\left(\epsilon^{-m u} x^{-\lambda} g(x, y) / y\right)^{\frac{1}{\lambda-\mu}} \\
Y & =\epsilon^{-\mu} g(x, y) X^{-\mu} .
\end{aligned}
$$

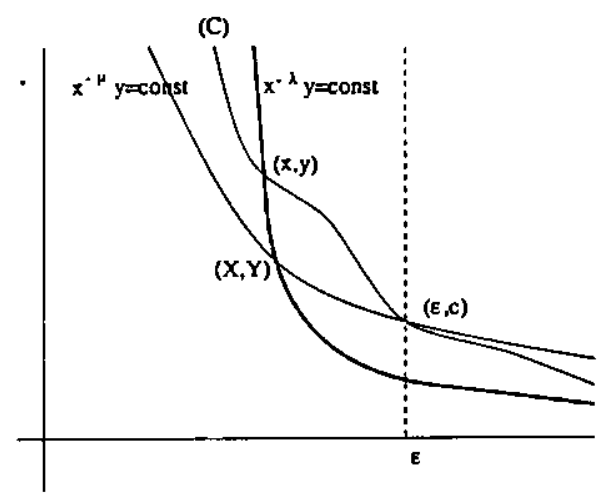

Figura 4.3: Andando ao longo das folhas de $\alpha$

Usando as propriedades da função $g$ em [6] podemos mostrar que $X$ e $Y$ estão bem definidos e que $(X, Y)$ é um homeomorfismo no primeiro quadrante. A mesma construção é feita para os demais quadrantes. O homeomorfismo construído é de fato um difeomorfismo fora dos eixos coordenados. Além disso podemos fixar $\lambda= \pm 1$ usando o homeomorfismo $\left(\operatorname{sign}(x) \cdot|x|^{-|\lambda|}, y\right)$.

O caso onde as separatrizes são comuns e os autovalores coincidem (esta é uma condição necessária para as 1-formas serem transversais fora da separatriz comum) é dado em [7]. Quando 
temos um par de 1-formas singulares com uma separatriz em comum e autovalores distintos, podemos supor que

$$
\begin{aligned}
j^{1} \alpha & =x d y-\lambda y d x \\
j^{1} \beta & =(x+y) d y-\mu y d x .
\end{aligned}
$$

O 2-jato do discriminante é dado por $y((\lambda-\mu) x+\lambda y)$. Desde que $\lambda-\mu \neq 0$, o discriminante tem uma singularidade $A_{1}^{-}$com um ramo das separatrizes em comum e o outro do Tipo 1, o qual nós assumimos ser transversal as separatrizes de $\alpha$ e $\beta$. Portanto temos o seguinte resultado:

Teorema 4.3.6 Sejam $\alpha$ e $\beta$ dois germes de 1-formas singulares com uma separatriz comum $e$ suponha que os autovalores dos campos de vetores associados às 1-formas são distintos. Suponha ainda que um dos ramos do discriminante, aquele formado por pontos do Tipo 1 , é transversal às separatrizes das 1 -formas diferenciais. Então existe um homeomorfismo levando o par $(\alpha, \beta)$ ao par

$$
(x d y \pm y d x,(x+y) d y-\mu y d x), \mu \neq 0
$$

A prova deste teorema é feita como a prova do Teorema 4.4.6 abaixo e usa o Teorema 4.3.5.

\subsection{Classificação topológica de pares de folheações no plano}

Apresentamos aqui modelos topológicos para pares de 1-formas diferenciais no plano da forma regular/regular, regular/singular e singular/singular com singularidade do tipo sela, nó ou foco. Nosso estudo cobre todos os fenômenos de codimensão menor ou igual a 1, exceto aqueles que provêm da instabilidade de uma das 1-formas. Começamos pelos pares regulares e estáveis.

Teorema 4.4.1 Sejam $\alpha$ e $\beta$ dois germes de 1-formas regulares e estáveis. Entāo um par estável $(\alpha, \beta)$ é topologicamente equivalente a um dos seguintes pares:

(i) $(d x, d y)$ : se as duas 1-formas são transversais.

(ii) $\left(d y, d\left(y-x^{2}\right)\right.$ : se as duas formas têm contato 2 na origem.

(iii) $\left(d y, d\left(y+x y+x^{3}\right)\right)$ : se as duas 1-formas tem contato de ordem 3 na origem.

Demonstração: A prova segue da Proposição 1.4.3 e do estudo feito na seção sobre transversalidade e genericidade. Vimos na seção 4.1 que a codimensão dos pares de 1-formas no plano depende do número de condiçõés que definem o par de 1-formas. No caso dos pares estáveis, podemos ter pares definidos por até duas condições. Sendo assim, 3 é o maior contato possível. Mas neste caso temos apenas os pares acima, dados pelo Teorema 1.4.3.

Agora consideramos a situação onde uma das 1-forma é singular, digamos $\beta$. Suponha que a singularidade de $\beta$ é do tipo sela, nó ou foco. Então os casos estáveis ocorrem quando a folha 
da 1-forma regular é transversal as separatrizes da 1-forma singular, no caso da sela e nó. Neste caso o conjunto dos pontos críticos é do Tipo 1. Estes pares foram estudados por Davydov em [16]. Apresentamos uma demonstração diferente usando o método do blowing up polar.

Teorema 4.4.2 Seja $\alpha$ um germe de 1-forma regular na origem e $\beta$ um germe de 1 -forma com singularidade do tipo sela, nó ou foco na origem. Então o discriminante é uma curva suave. Suponha que esta curva é transversal às folhas de $\alpha$. Então existe um homeomorfismo levando as folhas de $(\alpha, \beta)$ às folhas de

$$
\begin{array}{ll}
(d y,(x-y) d y+x d x), & \text { se } \beta \text { possui singularidade do tipo sela, } \\
(d y,(x+1 / 8 y) d y+x d x), & \text { se } \beta \text { possui singularidade do tipo nó } e \\
(d y,(x+y) d y+x d x), & \text { se } \beta \text { possui singularidade do tipo foco. }
\end{array}
$$
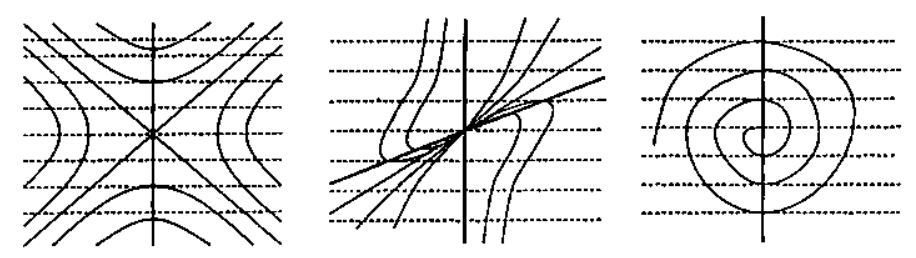

Figura 4.4: Pares de 1-formas regular/singular com discriminante transversal as folhas da 1-forma regular:regular/sela(à direita), regular/nó (centro) regular/foco(à esquerda)

Demonstração: Podemos supor que $\alpha=d y$ e que $j^{1}(\beta)=\left(a_{1} x+a_{2} y\right) d y+\left(b_{1} x+b_{2} y\right) d x$. Neste caso $\delta=b_{1} x+b_{2} y+O(2)$ é uma função suave, desde que a 1-forma é suave. Considerando mudanças de coordenadas da forma

$$
\psi(X, Y)=(\gamma X+\mu Y, \eta Y)
$$

com $\gamma \eta \neq 0$, temos as folhas de $\alpha$ preservadas e

$$
\begin{aligned}
\phi^{*}(\alpha) & =\left(\left(\eta a_{1} \gamma+\mu b_{1} \gamma\right) X+\left(\eta a_{1} \mu a_{2} \eta^{2} b_{1} \mu^{2}+b_{2} \mu \eta\right) Y\right) d Y \\
& +\left(\gamma^{2} b_{1} X+\left(\gamma \mu b_{1}+\gamma b_{2} \eta\right) Y\right) d X
\end{aligned}
$$

Assim se o discriminante é uma curva suave transversal as folhas de $\alpha$, temos que $\gamma \mu b_{1}+$ . $\gamma b_{2} \eta=0, \operatorname{com} \alpha \neq 0$. Agora se $b_{1}=0$ temos que uma separatriz de $\alpha$ tangencia uma folha de $\beta$ contradizendo a hipótese. Logo $b_{1} \neq 0$ e o primeiro jato do campo $\beta$ reduzido à

$$
j^{1}(x, y)=(x+\lambda y) d y+x d x
$$

Além disso para $1-4 \lambda>0$, o campo $\beta$ tem uma singularidade do tipo sela ou um nó na origem, dependendo do sinal de $\lambda$, e para $1-4 \lambda<0$ o campo $\beta$ tem uma singularidade do tipo foco na origem. Conseqüentemente podemos supor que

$$
\begin{aligned}
& \alpha=d y \\
& \beta=(x+\lambda y+A(x, y)) d y+(x+B(x, y)) d x
\end{aligned}
$$


onde $A$ e $B$ são funções suaves cujo 1-jato é identicamente nulo.

Aplicando o blow up polar $x=r \cos \theta$ e $y=r \operatorname{sen} \theta$ e dividindo as expressões por $r$ temos

$$
\begin{aligned}
\bar{\alpha} & =\operatorname{sen} \theta d r+r \cos \theta d \theta \\
\bar{\beta} & =\left(\cos ^{2} \theta+\cos \theta \operatorname{sen} \theta+\lambda \operatorname{sen}^{2} \theta+\phi_{1} \operatorname{sen} \theta+\phi_{2} \cos \theta\right) d r+ \\
& +r\left(\cos ^{2} \theta+(\lambda-1) \cos \theta \operatorname{sen} \theta+\phi_{1} \cos \theta-\phi_{2} \operatorname{sen} \theta\right) d \theta
\end{aligned}
$$

onde $\phi_{1}=(1 / r) A(r \cos \theta, r \operatorname{sen} \theta)$ e $\phi_{2}=(1 / r) B(r \cos \theta, r \operatorname{sen} \theta)$.

As singularidades de $\bar{\alpha}$ em $\{0\} \times S^{1}$ ocorrem para $\theta=0, \pi$, que são singularidades do tipo sela, e para $\bar{\beta}$, as singularidades ocorrem para os valores $\operatorname{de} \theta$ tais que

$$
\cos \theta^{2}+\cos \theta \operatorname{sen} \theta+\lambda \operatorname{sen} \theta^{2}=0 .
$$

Então se $\lambda<\frac{1}{4}$ (neste caso $\beta$ possui uma singularidade do tipo sela ou um nó na origem) existirão quatro valores de $\theta$ satisfazendo a equação 4.3. Além disso observe que se $\theta$ é solução da referida equação então $\theta+\pi$ também é.

$\bar{\beta}$ terá uma singularidade do tipo sela ou nó (serão quatro selas se $\lambda<0$ e duas selas e dois nós se $\left.0<\lambda<\frac{1}{4}\right)$.

Se $\lambda>\frac{1}{4}$ ( $\beta$ possui singularidade do tipo foco) a equação (4.3) não terá soluções reais, neste caso $\bar{\beta}$ não terá singularidades.

Vale notar que $\theta=0, \pi$ não satisfazem a equação (4.3), logo as singularidades de $\alpha$ e $\beta$ não coincidem em $\{0\} \times S^{1}$. Portanto localmente para cada um destes pontos o par pode ser reduzido por homeomorfismo ao modelo $(d y, x d y \pm y d x)$, como na Proposição 4.3.3.

Os demais pontos em $\{0\} \times S^{1}$ serão pontos regulares a ambas as formas.

A função discriminante de $(\bar{\alpha}, \bar{\beta})$ é dada por $\delta(r, \theta)=r \delta_{1}(r, \theta)$, onde $\delta_{1}(0, \theta)=-\cos \theta$.

Então dado $p \in\{0\} \times S^{1}-\left\{\frac{\pi}{2}, \frac{3 \pi}{2}\right\}$, temos $\delta_{1}(0, \theta) \neq 0$, ou seja $r=0$ é folha comum de ambas folheaçōes, sendo que fora desta curva as folhas são transversais, então temos um modelo local para o par de 1-formas numa vizinhança deste ponto dado pela Proposição 4.3.1, a saber $(d y,(1+x) d y+x d x)$.

Para $\theta=\frac{\pi}{2}$ ou $\frac{3 \pi}{2}$ temos $\delta_{1}(0, \theta)=0, \operatorname{mas} \frac{\partial \delta_{1}}{\partial \theta}(0, \theta)=\operatorname{sen} \theta-\frac{\partial}{\partial \theta}\left(\phi_{2}(0, \theta)\right)= \pm 1$. Logo $\alpha$ e $\beta$ são 1-formas regulares na vizinhança dos pontos $\left(0, \frac{\pi}{2}\right)$ e $\left(0, \frac{3 \pi}{2}\right)$ com discriminante com singularidade do Tipo Morse $A_{1}^{-}$com um ramo com pontos do Tipo 1, assim temos um modelo local pela Proposição 4.3.4.

Enfim temos modelos locais para cada ponto de $\{0\} \times S^{1}$ e utilizando as mesmas técnicas apresentadas por Bruce e Fidal em [6] para colagem temos um modelo local para um par de 1-formas numa vizinhança da origem.

Estudamos agora os fenômenos de codimensão 1. Quando as 1-formas são regulares, a degeneração pode ser medida pela ordem de contato na origem e pela singularidade do discriminante. 
Teorema 4.4.3 Sejam $\alpha$ e $\beta$ 1-formas regulares. Então os pares de codimensão 1 são topologicamente equivalentes a um dos seguintes pares:

(i) $\left(d y, d\left(y \pm x y^{2}+x^{3}\right)\right)$ : se as 1-formas têm contato 3 na origem e o discriminante tem uma singularidade do tipo Morse.

(ii) $\left(d y, d\left(y+x y+x^{4}\right)\right)$ : se as 1 -formas têm contato 4 na origem. Então o discriminante é uma curva suave.

Demonstração: A parte (i) do teorema segue como no Teorema 4.4.1 da Proposição 1.4.3. Passamos então ao estudo do caso (ii). Podemos supor que $\alpha=d y$ e $\beta=d f$, onde $f$ é germe de função suave. Começamos reduzindo este germe a forma $f=\varphi_{1}(y)+\varphi_{2}(y) x+\varphi_{3}(y) x^{2}+x^{4}$. Quando $f$ é regular podemos fixar $\varphi_{1}(y)=y$. O caso menos degenerado ocorre quando o discriminante é suave, ou seja, $\varphi_{2}(y)$ é regular, podemos então supor que $j^{1} \varphi(y)=y$. $O$ discriminante $\Delta$ tem uma inflexão e separa o plano em duas regiões .(ver Figura 4.5).

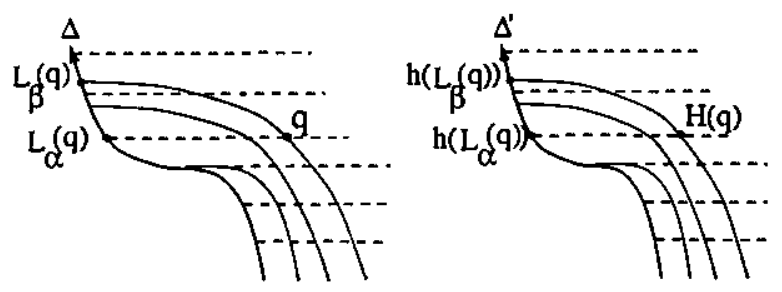

Figura 4.5:

Escolha uma região. Um ponto $q$ nesta região é unicamente determinado pela intersecção das folhas de $\alpha$ e $\beta$ passando pelo ponto $p$. Estas determinam dois pontos no conjunto discriminantes $\Delta, L_{\alpha}(q)$ e $L_{\beta}(q)$, respectivamente. Observe que $L_{\alpha}(q) \leq L_{\beta}(q)$ (se adotamos a orientação crescente para o discriminante, como na Figura 4.5).

Agora seja $\left.\beta^{\prime}=d\left(y+x y+x^{4}\right)\right)$ e $\Delta^{\prime}$ o discriminante do par $\left(\alpha, \beta^{\prime}\right)$, também orientado no sentido crescente. Escolha um homeomorfismo crescente $h$ de $\Delta$ para $\Delta^{\prime}$. Definimos $H(q)$ como o ponto de interseç̧ão da folha de $\alpha$ passando por $h\left(L_{\alpha}(q)\right)$ e a folha de $\beta^{\prime}$ passando por $h\left(L_{\beta}(q)\right)$. Note que $h$ é crescente então as duas folhas imagem interceptam-se em um único ponto na região escolhida.

Não é difícil verificar que $H$ é um homeomorfismo que leva as folhas do par $(\alpha, \beta)$ nas folhas do $\operatorname{par}\left(\alpha, \beta^{\prime}\right)$ na região escolhida. O mesmo homeomorfismo $h$ define $H$ de maneira análoga na outra região. Sendo assim temos o homeomorfismo desejado.

Um outro caso de codimensão 1 a considerar é quando uma das 1-formas é singular e a folha da 1-forma regular na origem tem tangência ordinária com as separatrizes da 1-formas singular. Teorema 4.4.4 Seja $\alpha$ um germe de 1-forma regular na origem e $\beta$ um germe com singularidade do tipo sela, nó ou foco na origem. Suponha quie a folha de $\alpha$ na origem tem uma tangência 
ordinária com uma das separatrizes de $\beta$. Então existe um homeomorfismo levando o par acima ao modelo $\left(d\left(y-x^{2}\right), x d y-\lambda y d x\right)$. Veja a Figura 4.6 .
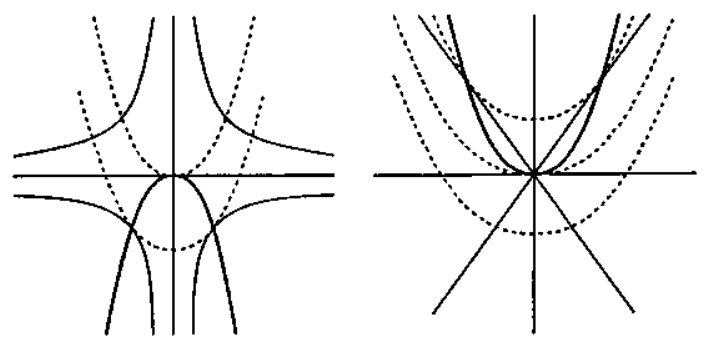

Figura 4.6: O caso não transversal: (regular, sela), à esquerda e (regular, nó) à direita

Demonstração: A prova deste teorema é análoga a prova do Teorema 4.4.2. Podemos fixar $\alpha=d\left(y-x^{2}\right)$ e reduzir o 1-jato de $\beta$ a $j^{1} \beta=(x+a y) d y-\lambda y d x$. A diferença entre esta demonstração com a demonstração do teorema anterior é que quando consideramos o blow up polar de dois zeros do levantamento de $\alpha$, os pontos singulares do levantamento do discriminante e os dois zeros do levantamento de $\beta$ coincidem. Mas os autovalores do campos de vetores associados aos levantamentos de $\alpha$ e $\beta$ são distintos em tais pontos e as duas 1-formas tem somente uma separatriz em comum, chamada de fibra excepcional. Usando então o Teorema 4.3.6, obtemos um modelo topológico em tais pontos. Faça agora como no Teorema 4.4.2. Desde que o modelo topológico não depende de $a$ podemos fixar $a=0$.

Supondo agora que as 1-formas são ambas singulares, vamos primeiramente analisar o 1-jato dos pares de 1-formas. Este caso é, em geral, de codimensão 2 (ver seção 4.1). Temos dois casos a considerar:

(I) $\alpha$ possui singularidade do tipo sela ou nó: Podemos fixar $j^{1} \alpha=x d y-\lambda y d x$ e reduzir o 1 -jato de $\beta$ à forma $j^{1} \beta=(x+\mu y) d y-(x+\gamma y) d x$. O polinômio característico da matriz associada ao $j^{1} \beta$ é dado por $t^{2}-(\gamma+1) t+(\gamma-\mu)$. Assim os sinais de $\delta_{1}=(\gamma-1)^{2}+4 \mu \mathrm{e}$ $\gamma-\mu$ que determinam o tipo de singularidade de $\beta$. Se $(\gamma-1)^{2}+4 \mu>0$ e $\gamma-\mu<0, \beta$ terá singularidade do tipo sela, se $(\gamma-1)^{2}+4 \mu>0$ e $\gamma-\mu>0, \beta$ terá singularidade do tipo nó. Para $(\gamma-1)^{2}+4 \mu<0, \beta$ terá singularidade do tipo foco.

Também temos que considerar a singularidade do discriminante.

O 2-jato do discriminante é dado por $-x^{2}+(\lambda-\gamma) x y+\lambda \mu y^{2}$. Supondo que a singularidade do discriminante é do tipo Morse, temos que esta é do tipo $A_{1}^{+}$( $\Delta$ é um ponto isolado) se $(\lambda-\gamma)^{2}+4 \lambda \mu<0$ e $A_{1}^{-}$( $\Delta$ é formado por retas transversais) se $(\lambda-\gamma)^{2}+4 \lambda \mu>0$.

Considerando as parábolas $(\gamma-1)^{2}+4 \mu=0$ e $(\lambda-\gamma)^{2}+4 \lambda \mu=0$ e a reta $\gamma-\mu=0$ no plano $(\gamma, \mu)$ para $\lambda$ fixo, podemos deduzir os vários pares de singularidades que podem ocorrer com um tipo particular de singularidade do tipo Morse para o discriminante, como mostramos 
na Figura 4.7.

(II) $\alpha$ e $\beta$ possuem singularidade do tipo foco: Podemos fixar $\alpha=(x-\lambda y) d y-(\lambda x+y) d x$. O 1-jato de $\beta$ pode ser escrito na forma $j^{1} \beta=(x+\mu y) d y-(x+\gamma y) d x, \operatorname{com}(\gamma-1)^{2}+4 \mu<0$. Assim o 2-jato do discriminante será dado por

$$
(\lambda-1) x^{2}+(\lambda \mu+\lambda-\gamma+1) x y+(\lambda \gamma+\mu) y^{2}
$$

e a singularidade do discriminante será do tipo $A_{1}^{+}$se $\delta_{2}=(\lambda \mu+\lambda-\gamma+1)^{2}-4(\lambda-1)(\lambda \gamma+\mu)<0$ e, do tipo $A_{1}^{-}$se, $\delta_{2}>0$. Veja na Figura 4.7 à esquerda as curvas que definem o tipo de singularidade de $\beta$ e o tipo de singularidade do discriminante.

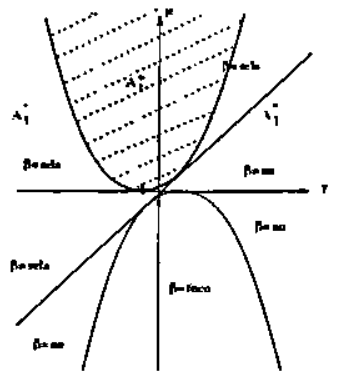

a w sela

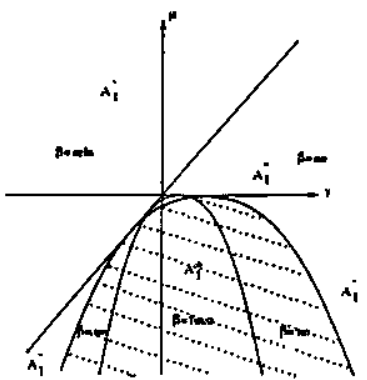

$\alpha=n$

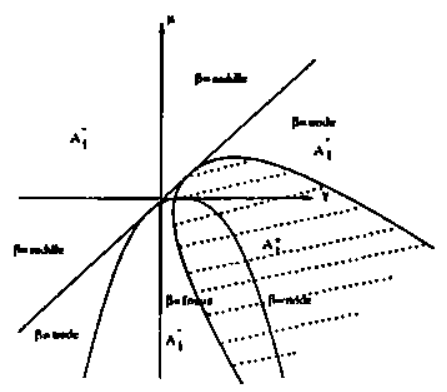

$\alpha=1000$

Figura 4.7: Curvas que determinam o tipo de singularidade do par $(\alpha, \beta)$

Conseqüentemente temos a seguinte proposiçāo:

Proposição 4.4.5 Modelos para todos os casos possíveis de pares de 1-jatos de 1-formas diferenciais com singularidade do tipo sela, nó ou foco na origem e que não possuem uma folha comum são apresentados a seguir:

I. Se o discriminante tem uma singularidade do tipo $A_{1}^{+}$:

$$
\begin{aligned}
(\text { sela, selo }): & (x d y+y d x,(x+y) d y-(x-y) d x) \\
(\text { nó, nó) }: & \left(x d y-2 y d x,\left(x-\frac{1}{8} y\right) d y-(x+2 y) d x\right) \\
(\text { nó, foco }): & (x d y-2 y d x,(x-y) d y-(x+2 y) d x) \\
(\text { foco }, \text { foco }): & ((x-2 y) d y-(2 x+y) d x,(x-y) d y-(x+y) d x)
\end{aligned}
$$
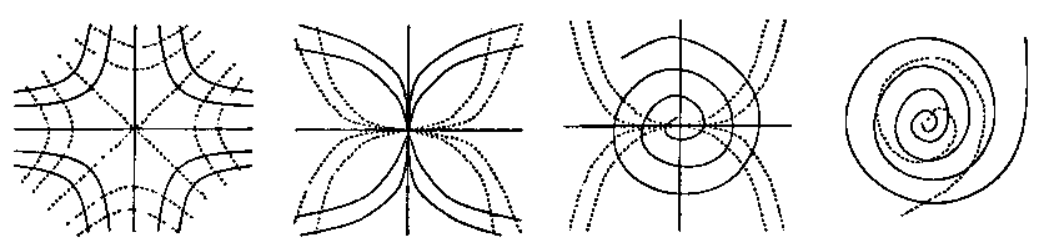

Figura 4.8: $\operatorname{Par}(\alpha, \beta)$ singular/singular cujo discriminante possui singularidade $A_{1}^{+}$ II. Se o discriminante tem uma singularidade do tipo $A_{1}^{-}$: 


$$
\begin{aligned}
(\text { sela,sela }): & \left(x d y+y d x,\left(x-\frac{1}{2} y\right) d y-(x-y) d x\right) \\
(\text { sela,nó) : } & (x d y+y d x,(x+y) d y-(x+2 y) d x) \\
(\text { sela,foco }): & (x d y+y d x,(x-y) d y-(x+y) d x) \\
(\text { nó,nó) : } & (x d y-2 y d x,(x+y) d y-(x+2 y) d x) \\
(\text { nó,foco }): & \left(x d y-2 y d x,\left(x-\frac{1}{10} y\right) d y-(x+y) d x\right) \\
(\text { foco,foco }): & ((x-2 y) d y-(2 x+y) d x,(x-3 y) d y-(x+y) d x)
\end{aligned}
$$
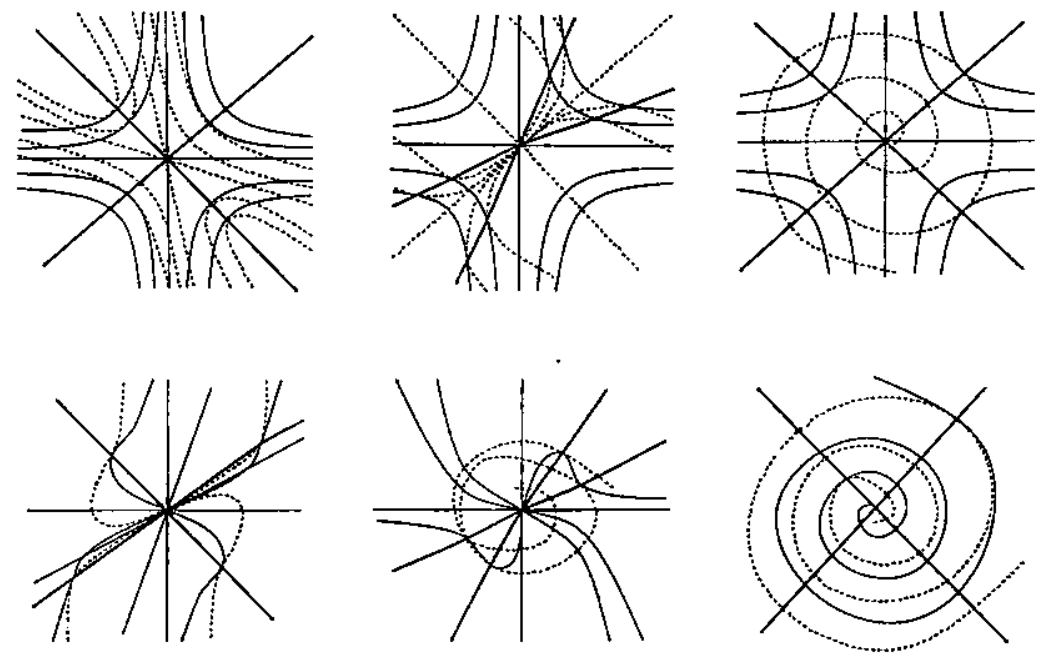

Figura 4.9: $\operatorname{Par}(\alpha, \beta)$ singular/singular cujo discriminante possui singularidade $A_{1}^{-}$

Podemos demonstrar então o seguinte teorema.

Teorema 4.4.6 Sejam $\alpha$ e $\beta$ 1-formas singulares sem folhas em comum com exceção da origem e com separatrizes transversais. Suponha que o conjunto discriminante tenha uma singularidade do tipo Morse $\left(A_{1}^{-}\right.$ou $\left.A_{1}^{+}\right)$com ramos transversais as separatrizes de $\alpha$ e $\beta$ no caso $A_{1}^{-}$. Então existe um homeomorfismo que leva as folhas do par $(\alpha, \beta)$ às folhas de um dos pares de 1-formas diferenciais dados na Proposição 4.4.5.

Demonstração: Supondo que $\alpha$ possui uma singularidade do tipo sela ou nó na origem temos $\alpha=\left(x+\phi_{1}(x, y)\right) d y-\left(\lambda y+\phi_{2}(x, y)\right) d x$, onde $\phi_{i}$ são funções suaves com 1-jato nulo. Além disso podemos escrever $\beta=\left(x+\tau y+\psi_{1}(x, y)\right) d y+\left(x+\gamma y+\psi_{2}(x, y)\right) d x$, onde $\psi_{i}$ assim como $\phi_{i}$ são funções suaves còm 1-jato nulo.

Aplicando o blowing up polar: $x=r \cos \theta, y=r \operatorname{sen} \theta$ e dividindo as 1-formas por $r$, teremos:

$$
\begin{aligned}
\bar{\alpha} & =\left((1-\lambda) \cos \theta \operatorname{sen} \theta+\overline{\phi_{1}} \operatorname{sen} \theta+\overline{\phi_{2}} \cos \theta\right) d r+ \\
& +r\left(\cos ^{2} \theta+\lambda \operatorname{sen}^{2} \theta+\phi_{1} \cos \theta+\phi_{2} \operatorname{sen} \theta\right) d \theta \\
\bar{\beta} & =\left((1+\gamma) \cos \theta \operatorname{sen} \theta+\tau \operatorname{sen}^{2} \theta+\cos ^{2} \theta+\overline{\psi_{1}} \operatorname{sen} \theta+\overline{\psi_{2}} \cos \theta\right) d r \\
& +r\left(\tau \operatorname{sen}^{2} \theta+(1+\gamma) \cos \theta \operatorname{sen} \theta+\cos ^{2} \theta+\overline{\psi_{1}} \cos \theta-\overline{\psi_{2}} \operatorname{sen} \theta\right) d \theta
\end{aligned}
$$


onde $\overrightarrow{\phi_{i}}=r \phi_{i}$ e $\overline{\psi_{i}}=r \psi_{i}$. Assim temos que as singularidades de $\bar{\alpha}$ em $0 \times S^{1}$ ocorrem para $\theta=0, \frac{\pi}{2}, \pi, \frac{3 \pi}{2}$, enquanto que para $\bar{\beta}$ ocorrem quando

$$
\tau \operatorname{sen}^{2} \theta+(1+\gamma) \cos \theta \operatorname{sen} \theta+\cos ^{2} \theta=0 .
$$

Portanto $\bar{\alpha}$ e $\bar{\beta}$ não possuem singularidades em comum.

Suponha agora que $\beta$ tem uma singularidade do tipo sela ou nó na origem então $(1+\gamma)^{2}-4 \tau=$ $(1-\gamma)^{2}-4(\tau-\gamma)>0$, o que implica que $\bar{\beta}$ terá singularidade em $0 \times S^{1}$. Enquanto que se $\beta$ tem uma singularidade do tipo foco então $\bar{\beta}$ é regular, pois $(1+\gamma)^{2}-4 \tau<0$ o que implica que a equação (4.4) não admite solução real.

Quanto ao discriminante do par $(\alpha, \beta)$ temos

$$
j^{2}(\delta(x, y))=x^{2}+(\gamma+\lambda) x y+\lambda \tau y^{2}
$$

ou seja, a singularidade do discriminante depende da equação $(\gamma+\lambda)^{2}-4 \lambda \tau$. Se $(\gamma+\lambda)^{2}-$ $4 \lambda \tau<0$ temos uma singularidade isolada para o discriminante, caso contrário, temos duas retas concorrentes.

Após o blowing up polar vemos que $\delta(r, \theta)=r \delta_{1}(r, \theta)$, sendo que

$$
\delta_{1}(0, \theta)=-\left(\cos ^{2} \theta+(\gamma+\lambda) \cos \theta \operatorname{sen} \theta+\lambda \tau \operatorname{sen}^{2} \theta\right)
$$

Portanto se $\Delta$ é um ponto isolado temos $\delta_{1}(0, \theta) \neq 0, \forall \theta$, ou seja, o discriminante de $(\bar{\alpha}, \bar{\beta})$ é regular e consiste em $0 \times S^{1}$. Mas se o discriminante é formado por um par de retas concorrentes (singularidade do tipo $A_{1}^{-}$), existirão valores de $\theta_{i}$ para os quais $\delta_{1}\left(0, \theta_{i}\right)=0$. Note porém que para estes pontos, temos

$$
\frac{\partial \delta_{1}}{\partial \theta}\left(0, \theta_{i}\right)=(\lambda+\gamma) \cos ^{2} \theta_{i}+2(\lambda \tau-1) \cos \theta_{i} \operatorname{sen} \theta_{i}-(\lambda+\gamma) \operatorname{sen}^{2} \theta_{i}
$$

Então $\frac{\partial \delta_{1}}{\partial \theta}$ se anula se, e somente se, $(\lambda \tau-1)^{2}+(\lambda-\gamma)^{2}=0$, no entanto temos as seguintes desigualdades:

$$
(\lambda \tau-1)^{2}+(\lambda-\gamma)^{2}>(\lambda+\gamma)^{2}-4 \lambda \tau>0
$$

a última desigualdade vinda da hipótese sobre a singularidade do discriminante do par $(\alpha, \beta)$ ser do tipo $A_{1}^{-}$. Portanto a derivada de $\delta_{1}$ em relação a $\theta$ não se anula, mostrando que os pares obtidos por blowing up também têm discriminante com singularidade do tipo Morse.

Vamos estudar agora todos os pontos sobre $0 \times S^{1}$. Para os pontos $(0, \theta) \operatorname{com} \theta=0, \frac{\pi}{2}, \pi, \frac{3 \pi}{2}$ ou, para $\theta$ satisfazendo a equaçāo (4.4) temos um campo singular e outro regular, cujo discriminante é uma folha comum, logo temos um modelo pela Proposição 4.3.3.

Para os demais valores de $\theta$ temos duas 1-formas regulares, cujo conjunto $\Delta$ é uma folha comum as 1-formas $(r=0)$, ou possui uma singularidade do tipo Morse com uma folha comum 
ao par, então pela Proposição 4.3.2 temos um modelo local para o primeiro caso enquanto que a Proposição 4.3.4, nos dá modelo local para o segundo caso.

Utilizando as técnicas apresentadas por Bruce e Fidal em [6, Proposição 4.1], colamos os modelos encontrados obtendo assim um modelo na vizinhança de $0 \times S^{1}$. Aplicando o blowing down teremos um modelo numa vizinhança da origem para o par de 1-formas diferenciais.

Se $\alpha$ e $\beta$ possuem singularidades do tipo foco na origem temos

$$
\begin{aligned}
& \alpha=\left(x-\tau y+\phi_{1}(x, y)\right) d y-\left(\tau x+y+\phi_{2}(x, y)\right) d x \\
& \beta=\left(x+\gamma y+\varphi_{1}(x, y)\right) d y+\left(x+\lambda y+\varphi_{2}(x, y)\right) d x
\end{aligned}
$$

Aplicando blowing up polar encontramos, após a divisão por $r$, as seguintes formas:

$$
\begin{aligned}
\bar{\alpha} & =\left(\tau+\overline{\phi_{1}} \operatorname{sen} \theta+\overline{\phi_{2}} \cos \theta\right) d r+\left(r+\overline{\phi_{1}} \cos \theta+\overline{\phi_{2}} \operatorname{sen} \theta\right) d \theta \\
\bar{\beta} & \left.=(1+\gamma) \cos \theta \operatorname{sen} \theta+\lambda \operatorname{sen}^{2} \theta+\cos ^{2} \theta+\overline{\varphi_{1}} \operatorname{sen} \theta+\overline{\varphi_{2}} \cos \theta\right) d r \\
& +r\left(\cos ^{2} \theta+(\lambda-1) \cos \theta \operatorname{sen} \theta-\gamma \operatorname{sen}^{2} \theta+\overline{\varphi_{1}} \cos \theta-\overline{\varphi_{2}} \operatorname{sen} \theta\right) d \theta
\end{aligned}
$$

Logo em $0 \times S^{1}, \alpha$ é regular e $\beta$ tem uma singularidade para os valores de $\theta$ que satisfazem a equação (4.4).

Vamos agora estudar os discriminantes dos pares $(\alpha, \beta)$ e $(\bar{\alpha}, \bar{\beta})$, como fizemos no caso anterior. Se o discriminante do par $(\alpha, \beta)$ tem singularidade isolada $\left(A_{1}^{+}\right)$, então $(1+\lambda)^{2}-4 \gamma<0$, logo o discriminante do $\operatorname{par}(\bar{\alpha}, \bar{\beta})$ é da forma $\delta(r, \theta)=r \delta_{1}(r, \theta)$, com $\delta_{1}(0, \theta) \neq 0$, ou seja, regular. Enquanto que se o discriminante é formado por um par de retas concorrentes $\left(A_{1}^{-}\right)$teremos valores para $\theta_{i}$ tais que $\delta_{1}\left(0, \theta_{i}\right)=0$, porém para tais valores de $\theta_{i}, \frac{\partial \delta_{1}}{\partial \theta}\left(0, \theta_{i}\right) \neq 0$, usando a hipótese dada pelo discriminante do $\operatorname{par}(\alpha, \beta)$.

Como $\alpha$ não possui singularidades temos que $(0, \theta)$ poder ser um ponto regular de $\alpha$ e singular de $\beta$, ou um ponto regular para ambas as 1-formas. No primeiro caso temos um modelo local para o par de 1-formas dado pela Proposição 4.3.3, desde que nestes pontos o discriminante é formado por uma folha comum. No caso de pares regulares temos pares regulares com folha comum, que aparecem se o discriminante do par $(\alpha, \beta)$ é um ponto isolado, logo temos modelos locais para tais pares dado pela Proposição 4.3.2. Ou ainda, temos pares regulares com singularidade do tipo Morse, neste caso temos um modelo local pela Proposição 4.3.4.

Logo conhecemos modelos locais para todos os pares de 1-formas sobre os pontos do círculo $0 \times S^{1}$. Usando as técnicas de colagem apresentadas por Bruce e Fidal [6], temos um modelo topológico para o par de 1-formas na vizinhança de $0 \times S^{1}$. Aplicando o blowing down, temos modelos numa vizinhança de $\mathbb{R}^{2}-\{0\}$. 


\section{Capítulo 5}

\section{Classificação formal de pares de folheações no plano}

Neste capítulo fazemos um estudo formal dos pares de germes de 1-formas diferenciais no plano. $\mathrm{O}$ método usado é a redução formal por séries de potências.

Este é um método devido à Poincaré, consiste em eliminar sucessivamente os termos de ordem $k(k \geq 2)$ nas funções que definem uma 1-forma diferencial. Nem sempre é possível provar que este processo é convergente. No entanto, Arnold [1] afirma que, em geral, os primeiros jatos de um germe de 1-forma são suficientes para o estudo da configuração da 1-forma.

Considere a 1-forma diferencial $\alpha=A(x, y) d y+B(x, y) d x$. Supondo que o $(k-1)$-jato de $\alpha$ pode ser reduzido a forma linear, podemos escrever seu $k$-jato na forma

$$
j^{k}(\alpha)=\left(a x+b y+A_{k}(x, y)\right) d y+\left(c x+d y+B_{k}(x, y)\right) d x
$$

onde $A_{k}$ e $B_{k}$ são polinômios homogêneos de grau $k$.

Considere a mudança de coordenadas $H_{k}$ em $\mathbb{R}^{2}, 0$ da forma

$$
\begin{aligned}
& x=X+P_{k}(X, Y) \\
& y=Y+Q_{k}(X, Y)
\end{aligned}
$$

onde $P_{k}$ e $Q_{k}$ são polinômios homogêneos de grau $k$ em $X$ e $Y$. Então

$$
\begin{aligned}
& d x=\left(1+\frac{\partial P_{k}}{\partial X}\right) d X+\frac{\partial P_{k}}{\partial Y} d Y \\
& d y=\frac{\partial Q_{k}}{\partial X} d X+\left(1+\frac{\partial Q_{k}}{\partial Y}\right) d Y
\end{aligned}
$$

Supondo que após esta mudança de coordenadas, os termos homogêneos de grau $k$ no $j^{k} \alpha$ podem ser eliminados, temos:

$$
\begin{aligned}
& X\left(a \frac{\partial Q_{k}}{\partial X^{\prime}}+c \frac{\partial P_{k}}{\partial X}\right)+Y\left(b \frac{\partial Q_{k}}{\partial X}+d \frac{\partial P_{k}}{\partial X}\right)+c P_{k}+d Q_{k}=-B_{k}(X, Y) \\
& X\left(a \frac{\partial Q_{k}}{\partial Y}+c \frac{\partial P_{k}}{\partial Y}\right)+Y\left(b \frac{\partial Q_{k}}{\partial Y}+d \frac{\partial P_{k}}{\partial Y}\right)+a P_{k}+b Q_{k}=-A_{k}(X, Y)
\end{aligned}
$$


onde $A_{k}$ e $B_{k}$ denotam o $k$-jato de $A_{k}\left(X+P_{k}(X, Y), Y+Q_{k}(X, Y)\right)$ e $B_{k}\left(X+P_{k}(X, Y), Y+\right.$ $\left.Q_{k}(X, Y)\right)$, respectivamente. Note que estes $k$-jatos dependem apenas dos $k$-jatos de $A_{k}$ e $B_{k}$.

As equaçōes acima geram um sistema linear de $2 k+2$ equações com $2 k+2$ incógnitas:

$$
M_{k}\left(\begin{array}{c}
P_{k} \\
Q_{k}
\end{array}\right)=-\left(\begin{array}{c}
B_{k} \\
A_{k}
\end{array}\right)
$$

sendo que o determinante da matriz $M_{k}$ dependerá dos autovalores $\left(\lambda_{1}, \lambda_{2}\right)$ do campo de vetores associados à 1-forma diferencial $\alpha$. Logo se o sistema acima pode ser resolvido para todo $k$, obtemos um difeomorfismo que reduz a forma $\alpha$ na sua parte linear, mas se existem enumeráveis valores de $\lambda=\left(\lambda_{1}, \lambda_{2}\right)$ para os quais temos $\operatorname{det} M_{k}=0$, dizemos que existe um difeomorfismo formal reduzindo $\alpha$ à sua parte linear. Estes valores de $\lambda$ se chamam valores resonantes. Então se $\lambda$ é não-resonante $\alpha$ é formalmente equivalente a sua parte linear (ver [14]).

Existem outros métodos que garantem a convergência da redução formal de 1-formas diferenciais, por exemplo, Bruce e Tari em [11], demonstraram que se a matriz obtida na redução satisfaz uma desigualdade envolvendo o módulo da inversa dessa matriz, então o processo converge. $O$ que ocorre na prática é que, exceto para aquelas matrizes cujo módulo da matriz inversa possa ser calculado com certa facilidade, este método não é fácil de ser aplicado.

\section{;.1 Estudo Formal}

Primeiramente estudamos os pares regular/singular com lsingularidade do tipo sela, nó ou foco. Observamos que o modelo obtido coincide com o modelo topológico.

Teorema 5.1.1 Suponha que o 1 -jato da 1-forma $\beta$ se escrive na fórma $(x+\lambda y) d y-x d x$ e $\alpha$ todos os valores de $\lambda$ existe um difeomorfismo fomal levando paratrizes de $\beta$. Então para quase par $((x+\lambda y) d y+x d x, d y)$.

Demonstração: Nas condições do teorema podemos fixar

onde $A_{2}$ e $B_{2}$ são polinômios he $\left(x+\lambda y+A_{2}(x, y)\right) d y+\left(x+B_{2}(x, y)\right) d x$

$$
\begin{aligned}
& \qquad \begin{array}{l}
\alpha=d y \\
\beta=\left(x+\lambda y+A_{2}(x, y)\right) d y+\left(x+B_{2}(x, y)\right) d x
\end{array} \\
& \text { onde } A_{2} \text { e } B_{2} \text { são polinômios homogêneos de grau } 2 \text {, escritos na forma } A_{2}(x, y)=a_{02} x^{2}+a_{12} x y+ \\
& a_{22} y^{2}+O(2) \text { e } B(x, y)=b_{02} x^{2}+b_{12} x y+b_{22} y^{2}+O(2) \text {. Considerando a } \\
& \phi_{2}(X, Y) \text { dada por }
\end{aligned}
$$$$
\text { Considerando a mudança de variável }
$$$$
x=X+P_{2}(X, Y)
$$$$
y=Y+q_{0} Y^{2}
$$ 
onde $P_{2}(X, Y)$ é um polinômio homogêneo de grau 2 e $q_{0} \in \mathbb{R}$. Multiplique $\phi_{2}^{*}(X, Y)$ por $1+R_{1}(X, Y)$, onde $R_{1}=r_{0} X+r_{1} Y$ o que não altera a 1-forma. (Note que esta mudança preserva as folhas de $\alpha$ ).

Quando fazemos $j^{2}\left(\left(1+R_{1}\right) \phi_{2}^{*}(\beta)\right)=(X+\lambda Y) d Y+X d x$ encontramos as seguintes equações:

$$
\begin{aligned}
& P_{2}(X, Y)+\lambda q_{0} Y^{2}+X R_{1}(X, Y)+\lambda Y R_{1}(X, Y)=-\overline{A_{2}}(X, Y) \\
& P_{2}+X R_{1}(X, Y)+X \frac{\partial P_{2}}{\partial X}=-\overline{B_{2}}(X, Y)
\end{aligned}
$$

onde $\overline{A_{2}}(X, Y)=a_{02} X^{2}+a_{12} X Y+a_{22} Y^{2}$ e $\overline{B_{2}}(X, Y)=b_{02} X^{2}+b_{12} X Y+b_{22} Y^{2}$.

Equivalentemente, o sistema acima pode ser escrito na seguinte forma:

$$
\left(\begin{array}{llllll}
1 & 1 & 0 & 1 & 0 & 0 \\
0 & 1 & 2 & \lambda & 1 & 2 \\
0 & 0 & 1 & 0 & \lambda & 3 \lambda \\
3 & 0 & 0 & 1 & 0 & 0 \\
0 & 2 & 0 & 0 & 1 & 0 \\
0 & 0 & 1 & 0 & 0 & 0
\end{array}\right)\left(\begin{array}{l}
p_{0} \\
p_{1} \\
p_{2} \\
r_{0} \\
r_{1} \\
q_{0}
\end{array}\right)=\left(\begin{array}{l}
a_{02} \\
a_{12} \\
a_{22} \\
b_{02} \\
b_{12} \\
b_{22}
\end{array}\right)
$$

Este sistema tem solução se o determinante da matriz é não nulo, ou seja, se $\lambda \neq 0, \frac{2}{9}$. Então para $\lambda \neq 0, \frac{2}{9}$ o par $(\alpha, \beta)$ é equivalente ao par

$$
\begin{aligned}
& \alpha_{3}=d y \\
& \beta_{3}=\left(x+\lambda y+A_{3}(x, y)\right) d y-\left(x+B_{3}(x, y)\right) d x
\end{aligned}
$$

onde $A_{3}$ e $B_{3}$ são funções suaves cujo 2 -jatos são identicamente nulos.

Suponha que esta construçãó é feit́a para $k=2,3, \ldots, n-1$ e vamos estudar o caso $k=n$. Por hipótese de induçāo, podemos escrever $j^{n} \beta$ da forma

$$
j^{n} \beta=\left(x+\lambda y+A_{n}(x, y)\right) d y+\left(x+B_{n}(x, y)\right) d x,
$$

onde $A_{n}$ e $B_{n}$ sāo polinōmios homogêneos de grau $n$. Considere a transformação $\phi_{n}$ tal que

$$
\begin{aligned}
& x=X+P_{n}(X, Y) \\
& y=Y+q_{0} Y^{n}
\end{aligned}
$$

onde $P_{n}$ é um polinômio homogêneo de grau $n, q_{0} \in \mathbb{R}$. Multiplique $\phi_{n}^{*}(\beta)$ por $1+R_{n-1}$, sendo $R_{n-1}$ um polinômio homogêneo de grau $n-1$.

$$
\begin{aligned}
& \text { um polinômio homogêneo de grau } n-1 . \\
& \text { Da condição } j^{n}\left(\left(1+R_{n-1}\right) \phi_{n}^{*}(\beta)\right)=(X+\lambda Y) d Y+X d X \text {, temos as equaçöes } \\
& \qquad \begin{array}{c}
P_{n}+\alpha q_{0} Y^{n}+X R_{n_{1}}+\alpha Y R_{n-1}+n q_{0} Y^{n_{1}} X+X \frac{\partial P_{n}}{\partial y}=-\overline{A_{n}} \\
P_{n}+X \frac{\partial P_{n}}{\partial x}+x R_{n-1}=-\bar{B}_{n}
\end{array} \\
& \text { ande } \overline{A_{n}} \text { e } \overline{B_{n}} \text { são polinôtnios de grau } n \text { das séries de Taylor de } A_{n} \text { e } B_{n} \text {. O sistema linear acima }
\end{aligned}
$$




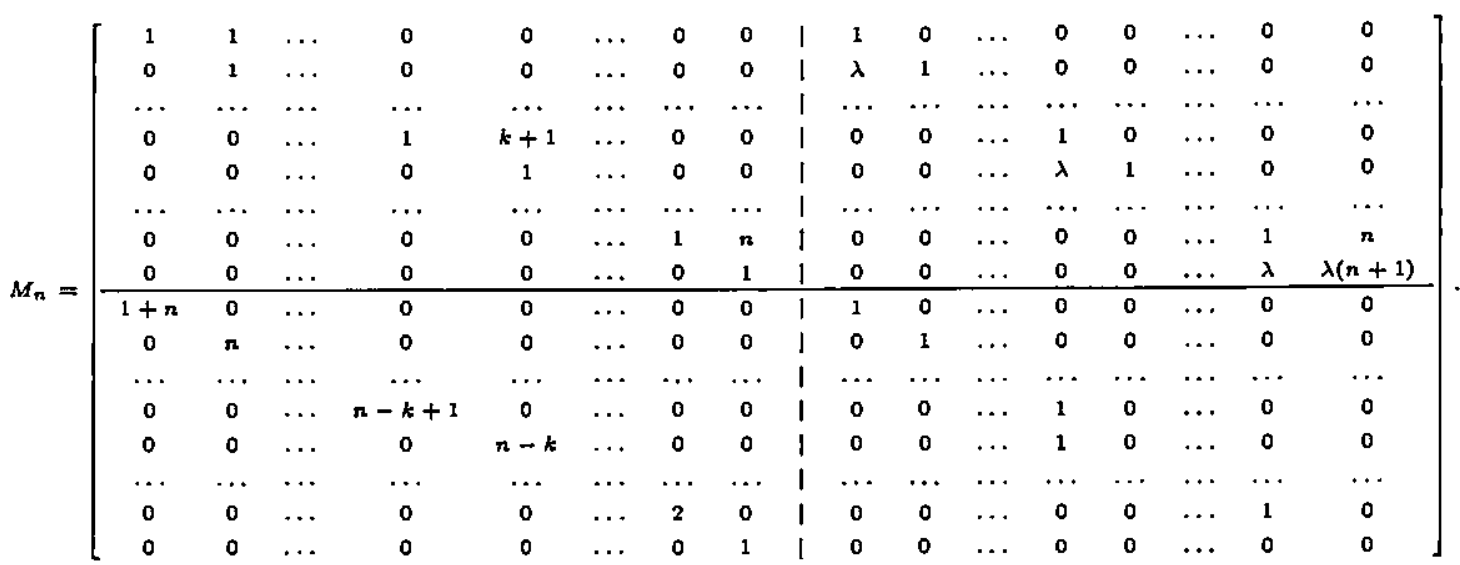

Vamos mostrar que o determinante desta matriz não é identicamente nulo se visto como um polinômio em $\lambda$. Observe que a matriz $M_{n}$ é formada por 4 blocos:

$$
\left(\begin{array}{ll}
A & B \\
C & D
\end{array}\right)
$$

onde $A, B, C, D$ são matrizes de ordem $n+1$, inversíveis, com exceção de $D$. Logo o $\operatorname{det} M_{n}=$ $\operatorname{det} A \cdot \operatorname{det}\left(D-C A^{-1} B\right)$. Além $\operatorname{disso} \operatorname{det}\left(M_{n}\right)$ é um polinômio em $\lambda$ de grau menor ou igual a $2 n+2$ e não é difícil observar que existe um coeficiente não nulo neste polinômio. Portanto para cada valor de $n$ o determinante se anula apenas para um número finito de valores de $\lambda$.

Considere o conjunto formado por todos os valores de $\lambda$ para os quais existe $n$ tal que a matriz acima possui determinante nulo. Este é um conjunto de medida nula. Exceto para $\lambda$ no conjunto acima, temos um difeomorfismo que conjuga o par de 1-formas ao modelo apresentado.

Aplicamos o mesmo método para pares singulares.

Teorema 5.1.2 Sejam $\alpha$ e $\beta$ duas 1-formas diferenciais com singularidades elementares na origem. Suponha que os 1-jatos de $\alpha$ e $\beta$ são dados pelas formas:

- $(x d y-\lambda y d x,(x+\mu y) d y-(x+\gamma y) d x)$, se $\alpha$ possue singularidade do tipo sela ou nó na origem;

- $((x-\gamma y) d y-(\gamma x+y) d x,(x+\mu y) d y-(x+\gamma y) d x)$, se $\alpha$ possue singularidade do tipo fóco na origem.

Então para quase todos os valores de $(\lambda, \gamma, \mu)$, existe um difeomorfismo formal que leva o par $(\alpha, \beta)$ aos pares:

- $(x d y-\lambda y d x,(x+\mu y+a(y)) d y-(x+\gamma y+b(y)) d x)$, se $\alpha$ possue singularidade do tipo sela ou nó na origem; 
- $((x-\gamma y) d y-(\gamma x+y) d x,(x+\mu y+a(y)) d y-(x+\gamma y+b(y)) d x)$, se $\alpha$ tem singularidade do tipo foco na origem,

onde a e b são séries formais com 1-jatos são nulos.

Demonstração: Vamos separar esta prova em dois casos. Caso 1: $\alpha$ possui singularidade do tipo sela ou nó. Caso 2: $\alpha$ tem singularidade do tipo foco.

Sejam $\alpha$ e $\beta$ como nas hipóteses do teorema e apliquemos a redução formal. No $n$-ésimo passo encontramos as seguintes equações

Caso 1:

$$
\begin{aligned}
& X \frac{\partial Q_{n}}{\partial X}+\lambda Y \frac{\partial P_{n}}{\partial X}+\lambda Q_{n}+\lambda Y R_{n_{1}}=-A_{n}(X, Y) \\
& X \frac{\partial Q_{n}}{\partial Y}+P_{n}+\lambda Y \frac{\partial P_{n}}{\partial Y}+X R_{n-1}=-B_{n}(X, Y) \\
& X \frac{\partial Q_{n}}{\partial X}+P_{n}+\mu Y \frac{\partial Q_{n}}{\partial X}+\gamma Q_{n}+X \frac{\partial P_{n}}{\partial X}+\gamma Y \frac{\partial P_{n}}{\partial X}+X S_{n-1}+\gamma Y S_{n-1}=-C_{n}(X, Y) \\
& X \frac{\partial Q_{n}}{\partial Y}+P_{n}+\mu Y \frac{\partial Q_{n}}{\partial Y}+\mu Q_{n}+X \frac{\partial P_{n}}{\partial Y}+\gamma Y \frac{\partial P_{n}}{\partial Y}+X S_{n-1}+\mu Y S_{n-1}=-D_{n}(X, Y)
\end{aligned}
$$

Caso 2:

$$
\begin{aligned}
& X \frac{\partial Q_{n}}{\partial X}-\lambda Y \frac{\partial Q_{n}}{\partial X}+P_{n}+\lambda Q_{n}+\lambda Y \frac{\partial P_{n}}{\partial X}+x \frac{\partial P_{n}}{\partial X}+X R_{n_{1}}+\lambda Y R_{n-1}=-A_{n}(X, Y) \\
& X \frac{\partial Q_{n}}{\partial Y}-\lambda Y \frac{\partial Q_{n}}{\partial Y}+P_{n}-\lambda Q_{n}+\lambda Y \frac{\partial P_{n}}{\partial Y}+X \frac{\partial P_{n}}{\partial Y}+X R_{n_{1}}-\lambda Y R_{n-1}=-B_{n}(X, Y) \\
& X \frac{\partial Q_{n}}{\partial Y}+P_{n}+\mu Y \frac{\partial Q_{n}}{\partial Y}+\mu Q_{n}+X \frac{\partial P_{n}}{\partial Y}+\gamma Y \frac{\partial P_{n}}{\partial Y}+X S_{n-1}+\mu Y S_{n-1}=-C_{n}(X, Y) \\
& X \frac{\partial Q_{n}}{\partial X}+P_{n}+\mu Y \frac{\partial Q_{n}}{\partial X}+X \frac{\partial P_{n}}{\partial X}+\gamma Y \frac{\partial P_{n}}{\partial X}+\gamma Q_{n}+X S_{n-1}+\gamma Y S_{n-1}=-D_{n}(X, Y)
\end{aligned}
$$

onde $A_{n}(X, Y)$ representa o $n$-jato de $A_{n}\left(X+P_{n}, Y+Q_{n}\right)$, analogamente para $B_{n}, C_{n}, D_{n}$.

As funções $R_{n}, S_{n}$ são devido a compensação dos paramêtros, feita pela multiplicação das equações acima por polinômios homogêneos de grau menor que $n$. Note porém que ainda não obtivemos um sistema com mesmo número de equações e incógnitas. Sendo assim igualamos o $n$-jato da 1-forma à 1-forma reduzida adicionado um termo näo linear em $y$.

Seguindo as idéias do teorema anterior, podemos mostrar que o determinante da matriz obtida é diferente de zero exceto em um conjunto de medida nula de $(\lambda, \gamma, \mu)$ para cada $n$. Logo o conjunto de valores para os quais o sistema deixa de ter solução é de medida nula e como no Teorema 5.1.1 segue o resultado. 


\section{Capítulo 6}

\section{Desingularização de pares de 1-formas diferenciais no plano}

Nosso objetivo neste capítulo é apresentar um teorema de desingularização para pares de germes de 1-formas diferenciais no plano.

Este teorema foi motivado pelos teoremas de desingularização de uma 1-forma diferencial apresentados por Seidenberg [34] e Dumortier [17].

\subsection{Desingularização de uma 1-forma diferencial}

Dada uma 1-forma analítica ou formal, Seidenberg [34] assegura que após um número finito de transformações da forma $x=X, y=X Y$ e $x=X Y, y=Y$, as singularidades da 1-forma se tornam simples, isto é, se $\lambda_{1}$ e $\lambda_{2}$ são os autovalores do campo de vetores associado a 1-forma, temos:

(i) $\lambda_{1} \cdot \lambda_{2} \neq 0$ e $\lambda_{1} / \lambda_{2} \notin \mathbf{Q}^{+}$ou

(ii) $\lambda_{1}=0$ e $\lambda_{2} \neq 0$.

As mudanças de coordenadas citadas acima são chamadas de blowing up direcional.

Dada uma 1-forma diferencial $\alpha$ analítica ou formal, podemos escrever $\alpha=A(x, y) d y+$ $B(x, y) d x$, onde $A$ e $B$ são funções analíticas ou definidas por séries formais, logo podemos definir a ordem da 1-forma por $o(\alpha)=\min \{\operatorname{grau} A, \operatorname{grau} B\}$, onde por grau entendemos a ordem da primeira derivada não nula da função avaliada em zero. Sendo assim podemos supor $o(\alpha)<\infty$. Como o blowing up diminui a ordem de $\alpha$, com finitos passos teremos $o(\alpha) \leq 1$.

Camacho, Lins Neto e Sad em [13] também estudaram a desingularização de campos de vetores no caso holomorfo e mostraram que finitas blowing ups reduzem a 1-forma na forma "desingularizada". Segundo [13], uma 1-forma $\alpha$ é desingularizada se as singularidades são 
simples, estão sobre uma linha projetiva invariante e o conjunto de separatrizes é constituído por curvas suaves e disjuntas; nenhuma separatriz passa por um "corner" (intersecção de dois divisores); todas as separatrizes são transversais ao divisor. Ou seja, eles analisaram também a configuração das separatrizes das 1-formas.

Temos ainda uma generalização dos estudos de Seidenberg [34] ao caso suave dado por Dumortier [17]. Dumortier estudou a decomposição das singularidades de um campo de vetores em uma variedade de dimensão 2, em singularidades mais simples. Para isso definiu o grau de degeneração de uma singularidade da seguinte maneira:

Dado um germe de campo de vetores em $\mathbb{R}^{2}$ com $j^{k} X(0)=0$ e $j^{k+1} X(0) \neq 0$, o grau de degeneração de $X$ em $0 \in \mathbb{R}^{2}$, denotado por $d X(0)$, é definido por

- $d X(0)=k$, se o $(k+1)$-jato de $X$ em 0 não é linearmente conjugado à $y^{k+1} \frac{\partial}{\partial x}$;

- $d X(0)=k+1$, caso contrário.

Dumortier [17] mostrou que por blowing up o grau de degeneração não aumenta. O problema para a desingularização surge então quando após blowing ups o grau de degeneração se mantém. Neste caso o campo de vetores é chamado fortemente dX(0)-preservado. Mas sob a condição do campo satisfazer a desigualdade de Lojasiewicz a redução das singularidades em finitos passos está garantida.

Um campo de vetores $X$ satisfaz a condição de Lojasiewicz se: $\exists l \in \mathbb{N}, c>0, \delta>0$ tal que $\|X(x)\| \geq c\|x\|\left\|^{l}, \forall\right\| x \| \leq \delta$. Todo campo de vetores analítico satisfaz tal desigualdade.

Segundo [17], se um campo de vetores satisfaz a desigualdade acima, suas curvas integrais possuem contato finito com curvas suaves. Blowing ups reduzem esse contato, logo após finitas blowing ups teremos transversalidade.

Dumortier define singularidades elementares, apresentando modelos topológicos para os campos que possuem tal singularidade. Em seguida afirma que dado um campo de vetores suave satisfazendo a desigualdade de Lojasiewicz, existe homeomorfismo que conjuga-o a um campo cujas singularidades são elementares. Neste artigo ainda, temos que a desigualdade de Lojasiewicz é equivalente a existência da desingularização após um número finito de blowing ups.

Com isso dada uma 1-forma diferencial plana $\alpha$, com singularidade isolada na origem, diremos que a singularidade de $\alpha$ é simples ou elementar se ela é topologicamente equivalente a uma das configurações da Figura 6.1, onde $H$ denota uma singularidade hiperbólica, $P$ uma singularidade parabólica, $C$ um corner(interseção de dois divisores) e $I$ um intervalo singular.

Neste capítulo utilizamos basicamente.o blowing up direcional, no entanto não é difícil estabelecer uma relação entre a blowing up direcional e a polar [17]:

Seja $\phi: S^{1} \times \mathbb{R} \rightarrow \mathbb{R}^{2}$ dada por $\phi(\theta, r)=(r \cos \theta, r \operatorname{sen} \theta)$ e $\psi: \mathbb{R}^{2} \rightarrow \mathbb{R}^{2}$ dada por $\psi(x, y)=(X, X Y)$. Defina $U \subset S^{1} \times \mathbb{R}^{2}$ onde $U=\{(\theta, r) ; \theta \in]-\frac{\pi}{2}, \frac{\pi}{2}[\}$. Considere $F: U \rightarrow \mathbb{R}^{2}$ 


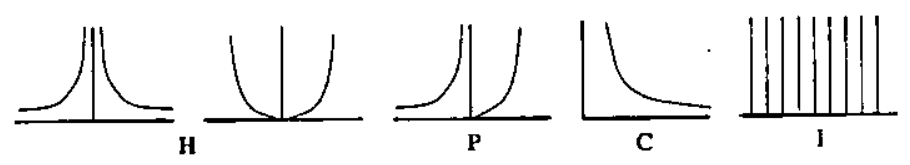

Figura 6.1: 1-formas com singularidade elementar

definida por $F(\theta, r)=(r \cos \theta, \tan \theta)$. $F$ é um difeomorfismo local de classe $C^{\infty}$ em $U$ que faz a equivalência entre o blowing up polar e o blowing up direcional, afinal é a mudança de coordenadas entre elas,

$$
(r \cos \theta, r \operatorname{sen} \theta)=(r \cos \theta, \tan \theta \cdot r \cos \theta)=(X, X Y)
$$

Nosso objetivo é mostrar que sob algumas condições, após um número finito de blowing ups um par de 1-formas diferenciais se torna desingularizado, num sentido que será definido abaixo.

\subsection{Desingularização de pares de 1-formas diferencias}

Dadas duas 1-formas diferenciais analíticas $\alpha$ e $\beta$ com finitas blowing up tornamos as singularidades de ambas simples ou elementares. Introduzimos a seguinte definiçāo para pares de 1-formas diferenciais:

Definição 6.2.1 Sejam $(\alpha, \beta)$ um par de 1-formas diferenciais analíticas/formais ou suaves, diremos que o par $\left(\alpha^{\prime}, \beta^{\prime}\right)$ obtido por blowing ups está desingularizado quando:

(1) as singularidades de $\alpha^{\prime}$ e $\beta^{\prime}$ forem elementares;

(2) o conjunto discriminante está desingularizado. Além disso o par $\left(\alpha^{\prime}, \beta^{\prime}\right)$ possue uma das seguintes configuraçōes nos pontos sobre o divisor excepcional:

(a) quando ambas forem regulares, o discriminante ou é formado pelo divisor excepcional ou um ramo dele é o divisor excepcional e outro consiste de pontos do Tipo 1;

(b) quando uma 1-forma é regular e a outra tem uma singularidade do tipo $H$ ou $P$ o discriminante é formado pelo divisor excepcional;

(c) quando ambas possuem singularidade do tipo $H$ ou $P$ o discriminante consiste das separatrizes dessas singularidades;

(d) quando uma das 1-formas é do tipo $I$, digamos $\alpha^{\prime}$, o discriminante é formado pelo divisor excepcional se $\beta^{\prime}$ é regular. Se $\beta^{\prime}$ tem singularidade do tipo $H$ ou $C$, então o discriminante é formado pelas separatrizes de $\beta^{\prime}$. Se $\beta^{\prime}$ é também do tipo $I$, então o discriminante consiste do divisor excepcional se as folhas de $\alpha^{\prime}$ e $\beta^{\prime}$ forem transversais ou tem contato finito entre si. Caso contrário o discriminante é formado pelo divisor excepcional e uma outra folha comum ao par.

Observemos que a definição acima é feita levando-se em conta a configuração do discriminante e das singularidades elementares das 1-formas. Em nosso caso, diferentemente do que apresentou 


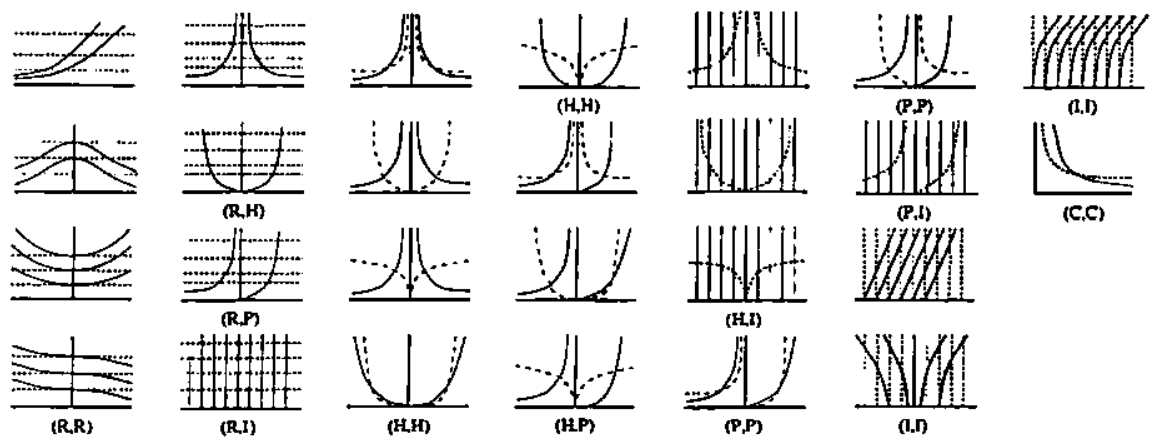

Figura 6.2: Pares de 1-formas desingularizadas

Dumortier em [17], não apresentamos modelos topológicos. Afinal não é claro se tais modelos existem para todas as configurações. Isso deve-se ao seguinte fato. Dizemos que um par possuem contato $(r, s)$ se o discriminante pode ser escrito em coordenadas locais na forma $\delta=x^{r} y^{s} \vec{\delta}$, onde $\bar{\delta}(0,0) \neq 0$. Aplicando um blowing up a esse par teremos um par cujo contato é $\left(r^{\prime}, s^{\prime}\right)$ com $r \leq r^{\prime}, s \leq s^{\prime}$ e $r+s \leq r^{\prime}+s^{\prime}$. Sabemos que a ordem de contato distingue pares regulares por difeomorfismos (Proposição 2.2, [30]) e pares do tipo sela/sela com mesmas separatrizes e autovalores resonantes (em [7]). Resta assim ser mostrado se a ordem de contato distingue ou não pares de 1-formas, topologicamente.

Estudaremos separadamente o caso analítico (o caso formal segue análogo) e o caso suave.

\subsubsection{O caso analítico}

Quando $\alpha$ e $\beta$ são 1-formas analíticas o conjunto discriminante $\Delta$ é uma curva plana analítica e portanto admite uma desingularização (ver [36]). Diremos que o discriminante $\Delta$ de $(\alpha, \beta)$, está desingularizado se, localmente, $\Delta$ é a pré-imagem do zero pela função $\delta(x, y)=x^{p} y^{q}$, onde $p, q \in \mathbb{N}$.

Temos o seguinte resultado:

Lema 6.2.2 Seja $(\alpha, \beta)$ um par de 1 -formas diferenciais analíticas/formais não idênticas, então existe um número finito de blowing ups do par que reduzem o discriminante à forma desingularizada.

Demonstração: Suponha $\alpha=a(x, y) d x+b(x, y) d y$ e $\beta=c(x, y) d x+d(x, y) d y$. Então $\delta(x, y)=\alpha \wedge \beta(x, y)=(a d-b c)(x, y)$.

Aplicando blowing up $x=X Y, y=Y$ ao $\operatorname{par}(\alpha, \beta)$ encontramos o par $(\bar{\alpha}, \bar{\beta})$ onde

$$
\begin{aligned}
& \bar{\alpha}=(a(X Y, Y)+X b(X Y, Y)) d Y+Y b(X Y, Y) d X \\
& \bar{\beta}=(c(X Y, Y)+X d(X Y, Y)) d Y+Y d(X Y, Y) d X .
\end{aligned}
$$

Logo o discriminante resultante é dado por

$$
\delta^{\prime}(X, Y)=\bar{\alpha} \wedge \bar{\beta}(X, Y)=Y(a d-b c)(X Y, Y)
$$


(O blowing up $x=X, y=X Y$ resulta em um discriminante da forma $X(a d-b c)(X Y, Y))$. Ou seja, a blowing up ao par de 1-formas resulta em blowing up do discriminante.

Desde que a função $a d-b c$ é analítica, temos, que após finitas blowing ups ao par, o discriminante resultante pode ser escrito em coordenadas locais na forma $x^{p} y^{q}=0$, portanto desingularizado.

Teorema 6.2.3 Dado um par de 1-formas diferenciais $(\alpha, \beta)$ analíticas/formais não idênticas, existe uma sequência finita de blowing up tal que o par $(\alpha, \beta)$ fica desingularizado no sentido da Definição 6.2.1.

Demonstração: Do teorema de Seidenberg temos que toda 1-forma diferencial analítica ou formal possue singularidades elementares após finitas blowing ups, então podemos supor que $\alpha$ e $\beta$ são 1-formas desingularizadas. Usando o Lema 6.2.2 também podemos supor que o discriminante deste par está desingularizado. Como aplicamos blowing ups simultâneas ao par podemos assumir que um dos ramos do discriminante é localmente o divisor excepcional (o que ocorre depois de uma blowing up), uma folha comum ao par de 1-formas. Isso já nos assegura que nada poderá ocorrer nos corners, afinal estes fazem parte do discriminante então o par é localmente transversal fora destes divisores, levando-nos a configuração como na Figura 6.2.

Com isso podemos supor que o par $(\alpha, \beta)$ é formado por 1-formas desingularizadas, seu discriminante está desingularizado e tem uma folha comum ao par de 1-formas. Passemos a análise dos possíveis pares de 1-formas.

(1) $\alpha$ e $\beta$ 1-formas regulares. Dadas as condições acima podemos supor que

$$
\begin{aligned}
& \alpha=d y \\
& \beta=d f
\end{aligned}
$$

onde $f: \mathbb{R}^{2}, 0 \rightarrow \mathbb{R}, 0$ é um germe de função analítica. Neste caso qualquer que sejam os pontos do discriminante temos que o par está desingularizado (independente do contato das folhas de $\beta$ com as folhas de $\alpha$ ).

(2) $\alpha$ regular e $\beta$ com singularidade do tipo $H$ ou $P$. Podemos escrever

$$
\begin{array}{ll}
\beta=x d y+\lambda y(1+A(x, y)) d x, & \operatorname{com} \lambda \neq 0, \text { se a singularidade é do tipo } H, \text { ou } \\
\beta=B(x, y) d y+\lambda y d x, & \operatorname{com} j^{1} B=0, \text { se a singularidade é do tipo } P .
\end{array}
$$

sendo que $y=0$ é o divisor excepcional. Também podemos supor que localmente temos

$$
\alpha=y(1+h(x, y))
$$

onde $\mathrm{h}$ é um germe de uma função analítica com $h(0,0)=0$. Então, em ambos os casos temos $\delta=y\left(\lambda+O_{1}(x, y)\right)$, ou seja, $y=0$ é localmente a única solução de $\delta=0$. Então o discriminante será formado apenas pelo divisor excepcional e o par está desingularizado por definição. 
(3) $\alpha$ e $\beta$ têm singularidade do tipo $H$ ou $P$. Como dissemos acima estamos assumindo que um dos ramos do discriminante é formado pelo divisor excepcional. Se o outro ramo do discriminante é uma separatriz comum ao par, entāo o par de 1-formas está desingularizado. Caso contrário, as separatrizes nāo comuns devem possuir contato finito entre si, afinal são curvas analíticas. Mostramos a seguir que blowing ups reduzem o contato das separatrizes. Desde que este número é finito pode ser reduzido a 1 e neste caso temos pares desingularizados.

(i) Se $\alpha$ e $\beta$ possuem singularidades do tipo $H$, podemos escrever

$$
\begin{aligned}
& \alpha=x d y+\lambda y(1+\mathrm{A}(x, y)) d x e \\
& \beta=(x+\mathrm{B}(x, y)) d y+\mu y d x
\end{aligned}
$$

Dessa maneira $\mathrm{B}(0, y)$ determina o contato da separatriz de $\beta$ em zero com $x=0$.

Aplicando blow up ao par obtemos:

$$
\begin{aligned}
& \bar{\alpha}=x(1+\lambda(1+\overline{\mathrm{A}}(x, y))) d y+\lambda y(1+y \overline{\mathrm{A}}(x, y)) d x \\
& \bar{\beta}=(x(1+\mu)+\overline{\mathrm{B}}(x, y)) d y+\mu y d x
\end{aligned}
$$

onde $\bar{A}(x, y)=A(x y, y) / y$ e $\bar{B}(x, y)=B(x y, y) / y$. Logo $\delta(x, y)=y((\lambda-\mu)+\bar{B}(x y, y)-$ $x A(x y, y)+D(x, y))$, onde $D(0,0)=0$.

Se $\lambda \neq \mu$, temos um novo par de 1-formas cujo discriminante é o divisor excepcional, $y=0$. Se $\lambda=\mu$, temos um par de 1-formas cujo discriminante contém o divisor excepcional. No entanto, em ambos os casos o contato entre as separatrizes é menor que do par original, desde que este depende de $\bar{B}(0, y)$. Desde que o contato é finito, aplicamos finitas blowing ups e obtemos pares cujas separatrizes serão transversais. Analisando a vizinhança de cada ponto sobre o divisor excepcional encontramos pares desingularizados: pares regulares, pares do tipo regular-hiperbólico e pares singulares hiperbólicos com separatrizes comuns. Logo o par se tornou desingularizado com finitas blowing ups.

(ii) Se $\alpha$ possue singularidade do tipo $H$ e $\beta$ do tipo $P$. Escrevemos

$$
\begin{aligned}
\alpha & =x d y+\lambda y(1+\mathrm{A}(x, y)) d x e \\
\beta & =B(x, y)) d y+\mu y d x
\end{aligned}
$$

Novamente $\mathrm{B}(0, y)$ determina o contato da separatriz de $\beta$ em zero com $x=0$ e $y=0$ é separatriz comum.

Aplicando blow up ao par obtemos:

$$
\begin{aligned}
& \bar{\alpha}=x(1+\lambda(1+\overline{\mathrm{A}}(x, y))) d y+\lambda y(1+y \overline{\mathrm{A}}(x, y)) d x \\
& \bar{\beta}=(\lambda x+\bar{B}(x, y)) d y+\mu y d x
\end{aligned}
$$

onde $\bar{A}(x, y)=A(x y, y) / y$ e $\bar{B}(x, y)=B(x y, y) / y$. Logo como no caso anterior temos que para $\lambda \neq \mu$ teremos um par cujo discriminante é apenas o divisor excepcional, sendo que para 
$\lambda=\mu$ temos o discriminante contém o divisor excepcional. Mas em ambos os casos temos que o par obtido por blowing up do par original tem uma separatriz comum e a outra separatriz com contato menor. Desde que o contato é finito em finitas blowing ups teremos um par com separatrizes comuns, logo desingularizado

(iii) Se $\alpha$ e $\beta$ têm singularidade do tipo $P$. Neste caso o procedimento é o mesmo dos casos anteriores, basta tomar

$$
\begin{aligned}
& \alpha=A(x, y) d y+\lambda y d x \\
& \beta=B(x, y) d y+\mu y d x .
\end{aligned}
$$

(4) Suponha que $\alpha$ tem uma singularidade do tipo $I$. Podemos supor que $\alpha$ é expressa localmente por

$$
\alpha=y^{p} d x
$$

Então se $\beta$ é regular, podemos assumir que as folhas de $\beta$ são curvas de nível de uma função diferenciável

$$
f(x, y)=y(1+h(x, y)) \quad \text { com } h(0,0)=0 .
$$

Com isso, o discriminante é dado por $y^{p}\left(1+h(x, y)+y h_{y}(x, y)\right)=0$, ou seja, o par está desingularizado (novamente independente do contato da folha de $\beta$ na origem com a separatriz de $\alpha$ ).

Se $\beta$ é singular com separatrizes transversais o par está desingularizado. Caso contrário, podemos escrever

$$
\begin{array}{ll}
\beta=(x+A(x, y)) d y+\lambda y d x, & \operatorname{com} \lambda \neq 0, \text { se a singularidade é do tipo } H, o u \\
\beta=B(x, y) d y+\lambda y d x, & \operatorname{com} j^{1} B=0, \text { se a singularidade é do tipo } P .
\end{array}
$$

Aplicando $x=X$ e $y=X Y$ temos

$$
\begin{array}{lll}
\bar{\beta} & =X d Y+Y(\lambda+\bar{A})(X, Y) d X, & \text { no primeiro caso ou } \\
\bar{\beta} & =X^{s}(1+\ldots) d Y+Y(\lambda+\bar{A}(X, Y)) d Y &
\end{array}
$$

onde $s$ é o grau de $B$. Em ambos os casos temos que o discriminante será dado pelos eixos $x=0$ e $y=0$. Portanto o par está desingularizado.

Suponha agora que $\beta$ tem singularidade do tipo $I$ então $\beta=y^{q} d f$, onde $f$ é um germe de função analítica regular. Então podemos supor que

$$
f(x, y)=x+\lambda y+h(x, y)
$$

Utilizando mudanças de coordenadas que preservam $y=0$ e as linhas verticais (ou seja, não alteram a configuração de $\alpha$ ), podemos escrever $f(x, y) \simeq x+y$, se $\lambda \neq 0$ e se $\lambda=0$, temos $f(x, y) \simeq x \pm y^{k}$ ou $f(x, y)=x+y \bar{h}(x, y)$. 
Assim temos os seguintes casos:

$$
\beta^{\prime}=y^{q} d(x+y)
$$

quando as direções limites das folhas de $\beta$ são transversais as folhas de $\alpha$, ou

$$
\beta^{\prime}=y^{q} d\left(x \pm y^{k}\right)
$$

quando as direções limites das folhas de $\beta$ possuem contato $k$ com as folhas de $\alpha$, ou ainda,

$$
\beta^{\prime}=y^{q} d(x+y \bar{h}(x, y))
$$

quando as folhas do par têm contato finito na origem.

Nos dois primeiros casos temos que o discriminante é dado pelo divisor excepcional, $y=0$, logo o par está desingularizado por definição.

Quanto ao terceiro caso temos $\delta=y^{p+q}\left(h(x, y)+y h_{y}(x, y)\right)$. Desde que por hipótese, $\delta$ está desingularizado, existem mudanças de coordenadas de modo que este pode ser escrito da forma $\delta(x, y)=y^{p^{\prime}}(x+k(x, y))^{q^{\prime}}$.

Blowing up da forma $x=X$ e $y=X Y$ ao par resulta na blowing up do discriminante, donde obtemos $\delta=y^{p^{\prime}} x^{\prime q}(1+\overline{k(x, y)})$. Neste caso obtivemos um par cujo discriminante é formado pelas separatrizes comuns, logo um par desingularizado.

\subsubsection{O caso suave}

No caso suave, assumindo que as 1-formas satisfazem a desigualdade de Eojasiewicz, o teorema de Dumortier [17] assegura que ambas as 1-formas podem ser desingularizadas. Para desingularizar o discriminante o qual é uma função suave, podemos, por exemplo, supor que a função que define-o é finitamente determinada, portanto é equivalente por mudanças de coordenadas a um polinômio. Assim o discriminante será uma curva algébrica, donde pode ser desingularizado.

Quanto ao par de 1-formas, precisamos considerar ainda o contato entre as folhas. No caso analítico o contato é finito, o que não necessariamente ocorre no caso suave. Duas curvas suaves podem ter contato infinito sem coincidirem. Considere por exemplo, o caso em que $\alpha=x d y-\lambda y d x$ e as folhas de $\beta$ são dadas pelas curvas de nível da função $y-f(x)$, onde $f$ possui todas as derivadas nulas em 0 . Então a folha de $\beta$ na origem tem contato infinito com a separatriz de $\alpha$. O discriminante é dado por $\lambda y-x f^{\prime}(x)=0$ que será desingularizado pois a função discriminante é 1-determinada.

Contudo se além da desigualdade de Eojasiewicz e da determinação finita da função discriminante supormos que as folhas do par de 1-formas têm contato finito, asseguramos a desingularização deste par pelo mesmo procedimento do caso analítico. Então podemos enunciar o seguinte teorema: 
Teorema 6.2.4 Sejam $\alpha$ e $\beta$ duas 1-formas diferenciais suaves satisfazendo a desigualdade de Lojasiewicz. Suponha que a função discriminante desse par de 1-formas é finitamente determinada e que suas separatrizes têm ordem de contato finito. Então existe a desingularização para $(\alpha, \beta)$.

Observação 6.2.5 Se provarmos que as configurações na Figura 6.2 são modelos topológicos, podemos usar os teoremas acima para obter uma classificação dos pares de folheações, onde dois pares são equivalentes se possuem a mesma configuração após a desingularização. 


\section{Observações finais e problemas}

\section{futuros}

Muitas questões interessantes surgiram no desenvolvimento deste trabalho que pretendemos investigar:

- Investigar se o processo da redução formal nos casos estudados na tese é convergente e de modo geral, estabelecer condições para que a redução formal implique na redução suave.

- Gutierrez (1986) mostrou que todo fluxo contínuo em uma variedade compacta de classe $C^{\infty} M$ é topologicamente equivalente a um fluxo $C^{1}$ sobre $M$, e sob determinadas condições, a um fluxo $C^{\infty}$. Seria interessante verificar se este resultado se generaliza para pares de folheações sobre $M^{2}$.

- Aplicar os resultados da tese à teoria de controle.

- Estudamos neste trabalho pares de folheaçōes em variedades bidimensionais com bordo regular, cujo bordo é transversal as folhas do par na origem ou então coincide com a folha de uma das folheações. Resta ainda investigarmos o caso onde ambas as folheações são tangentes ao bordo.

- Na desingularização dos pares de folheações no plano definimos os pares desingularizados. Uma questão natural é se esse pares são modelos topológicos. Se a resposta for positiva, podemos afirmam que dois pares que têm as mesmas configurações após a desingularização são topologicamente equivalentes.

- Durante o desenvolvimento deste trabalho observamos a importância do discriminante no estudo dos pares de folheações regulares no plano. Surgiu então uma questão bastante interessante, se as singularidades do discriminante determinam as classes topológicas de pares de 1-formas regulares no plano. 


\section{Referências Bibliográficas}

[1] V.I. Arnold, Geometrical Methods in the Theory of Ordinary differential equations, translated by Joseph Szücs, A Series of Comprehensive Studies in Mathematics Springer-Verlag New York Inc. (1983).

[2] V.I. Arnold, Ordinary Differential equations, Translated from the third Russian edition by Roger Cook, Springer Textbook, Springer-Verlag, Berlin (1992).

[3] V.I. Arnold, A. Varchenko, S. Gussein-Zade, Singularities of differentiable maps. Vol. 1. The classification of critical points, caustics and wave fronts. Monographs in Mathematics, Birkhuser Boston, Inc., 82, Boston, Mass., (1985).

[4] T. Banchoff, T. Gaffney, C. Mc Crory, Cusps of Gauss Mappings, Research Notes in Mathematics, 55, Pitman Publishing, Inc. (1982).

[5] T. Brocker, Differentiable Germs and Catastrophes, translated by L.Lander, London Society Lecture Note Series, Cambridge University Press, 17 (1975).

[6] J.W. Bruce and D.L. Fidal, On binary differential equations and umbilics, Proc. of Royal Society of Edinburgh, 111A (1989), 147-168.

[7] J.W. Bruce, G.J. Fletcher, F. Tari, Bifurcations of implicit differential equations, Proc. Royal Soc. Edinburgh 130A (2000), 1-22.

[8] J.W. Bruce, A. du Plessis, C.T.C Wall, Determinacy and unipotency, Inventiones Mathematicae, 88 (1987), 521-554.

[9] J.W. Bruce, N.P. Kirk and A.A. du Plessis, Complete transversals and the classification of singularities, Nonlinearity 10 (1997), No. 1, 253-275.

[10] J.W. Bruce and P. Giblin, Curves and singularities. A geometrical introduction to singularity theory, Cambridge University Press, Cambridge, 1992.

[11] J.W. Bruce and F. Tari, On binary differential equations, Nonlinearity, 8 (1995), 255-271. 
[12] J.W. Bruce and F. Tari, Implicit differential equations from the singularity theory viewpoint, in Singularities and Differential Equations (Warsaw, (1993), 23-38), Banach Centre Publication, 33, Polish Acad. Sci., Warsaw (1996).

[13] C. Camacho, A. Lins Neto and P. Sad, Topological Invariants and Equidesingularization for Holomorphic Vector Fields, J. Differential Geometry, 20 (1984), 143-174.

[14] K. Chen, Equivalence and decomposition of vector fields about elementary and singular point, Amer. J. Math., 85 (1963), 639-722.

[15] J.N. Damon, The unfolding and determinancy theorems for subgroups of $\mathcal{A}$ and $\mathcal{K}, \mathrm{Mem}$. Amer. Math. Soc. 50, 306 (1984).

[16] A.A. Davydov, Qualitive Theory of Control Systems, Transl. Mat. Monographs, AMS 141 (1994).

[17] F. Dumortier, Singularities of Vector Fields on the Plane, Journal of Diff. Equations, 23 (1977), 53-106.

[18] C.G. Gibson, Singular points of smooth mappings, Research Notes in Mathematics. Pitman (Advanced Publishing Program), 25, Boston, Mass.-London, 1979.

[19] M. Golybitsky and V. Guillemin, Stable mappings and their singularities. Graduate Text in Mathematics, GTM 14, Springer-Verlag, N.Y. (1973).

[20] C. Gutierrez and J. Sotomayor, Structurally stable configurations of lines of principal curvature, Astérique (1982), 98-99.

[21] P. Hartman, On local homeormorphisms of Euclidian spaces, Bol. Soc. Math. Mexican, 5 (1960), 220-241.

[22] B. Malgrange, Ideals of differentiable functions, Oxford University Press, (1966).

[23] S. Mancini, M. A. S. Ruas and M. A. Teixeira, On divergent diagrams of finite codimension. Preprint, (2000).

[24] J. Martinet, Singularities of smooth functions and maps, Cambridge University Press. LMS Lecture Notes 58 (1982).

[25] J.N. Mather, Stability of $C^{\infty}$ mappings. The division theorem. Ann. of Math. (2) 87 (1968), 89-104.

[26] J.N. Mather, Stability of $C^{\infty}$ mappings, II. Infinitesimal stability implies stability, Ann. of Math. (2) 89 (1969), 254-291. 
[27] J.N. Mather, Stability of $C^{\infty}$ mappings, III. Finitely determined map-germs, Publ. Math.I.H.E.S., 36 (1968), 127-156.

[28] J.N. Mather, Stability of $C^{\infty}$ mappings, IV. Classification of stable germs by $R$-algebras, Inst. Hautes Études Sci. Publ. Math. no. 37 (1969), 223-248.

[29] J.N. Mather, Stability of $C^{\infty}$ mappings, V. Transversality, Advances in Math. 4 (1970), 301-336.

[30] M. F. Michel, Modeles Locaux de Formes Differentielles Quadratiques, PhD Thesis, L'Universite Paul Sabatier de Toulouse (1994).

[31] J. Montaldi, On contact between submanifolds, Michigan Math. Journal, 33, 3 (1986), 195-199.

[32] The Liverpool Surface Modeling Package, written by R. J. Morris (rjm@ amsta.leeds.ac.uk) for Silicon Garphics and X windows. See R.J. Morris, The use of computer graphics for solving problems in singularity theory, in H. -C. Hege and K. Polthier (Eds), Visualization in Mathematics, Springer-Verlag, Heidelberg, 1997, 53-66.

[33] R. Garcia, J. Sotomayor, Structural Stability of Parabolic Points and Periodic Asymptotic Lines, Matematica Contemporânea, 12 (1997), 83-102. Sociedade Brasileira de Matemática, edited by Ruas, M.A.S., Workshop on Real and Complex Singularities, São Carlos, July, 22-26, 1996, Universidade de São Paulo, ICMSC-SP.

[34] A. Seidenberg, Reduction of Singularities of the Differential Equation $A d y=B d x$, Amer.J.Math., 90 (1968), 248-269.

[35] W. Szlenk, An introduction to the theory of Smooth Dynamical Systems - John Wiley \& Sons, PWIV - Polish Scientific Publishers, Warzawa (1984).

[36] B. Teissier, Introduction to Curve Singularities, in Singularity Theory (Trieste, 1991), World Sci. Publishing, River Edge, NJ (1995), 866-893.

[37] M. A. Teixeira, On structural stability of pairings of vector fields and functions, Bol. Soc. Bras. Mat., 9 (1978), 63-82.

[38] M. A. Teixeira, On the local first order structural stability of pairings of vector fields and functions, Czechoslovak Math. Journal, 30, 105 (1980), 228-249.

[39] C. T. C. Wall, Finitely determinacy os smooth map-germs,Bull. London Math. Soc., 13 (1981), 481-539. 


\section{E R R A T A}

Sobre pares de 1-formas diferenciais em variedades de dimensão 2

O objetivo dessa errata não é corrigir erros gramaticais, sei que neste trabalho muitos podem ser encontrados, mas mencionar um problema que apenas compreendi após a minha defesa de doutoramento, assim como conseqüências do mesmo neste trabalho.

Nos capítulos 1, 2 e 3 deste trabalho, na classificação dos pares de 1-formas, usamos mudanças de coordenadas que preservam as linhas horizontais, $y=c$, que representam as folhas da 1-forma regutar $\alpha=d y$. Primeiramente questionamos a existência dos teoremas fundamentais da teoria de singularidades para esse tipo de mudanças, o teorema da determinação finita e dos desdobramentos versais. Em seguida, para verificar esse fato usamos a definição de subgrupos geométricos apresentada por Damon.

No entanto, o grupo de mudança de coordenadas acima não definem um subgrupo geométrico. Isso pode ser justificado pela sua representação na forma de um diagrama divergente. A condiçāo essencial para um dado grupo ser um subgrupo geométrico é a existência de um sistema adequadamente ordenado de DA-álgebras. $\mathrm{O}$ que não acontece nos diagramas divergentes.

O problema neste trabalho foi a construção de um sistema de DA-algebras que não representa o tipo de mudanças de coordenadas desejadas, que preservam as retas horizontais.

Entre as conseqüências desse fato temos que a classificação dos pares de 1-formas diferenciais regulares ou (regular, singular-exata) em variedades de dimensão 2 é apenas formal (não mais suave como enunciado nesta tese). Mas observamos que podemos utilizar ainda outras ferramentas da teoria de singularidades para demonstrar, em certos casos, a existência de modelos suaves na classificação, como ocorre, por exemplo, nos pares com contato 1 e 2. Podemos ainda utilizar a classificação formal obtida para a construção de uma classificação topológica, além do estudo das 
deformaçōes dos pares classificados.

Devido a regras sobre trabalhos entregues à $\mathrm{CPG}$, que não permite atualização dos mesmos, àqueles que tiverem interesse em ler este trabalho revisado, solicitamos que entrem em contato conosco, será um prazer enviar-lhe a versão revisada. O endereço para contato se encontra na seção de pós-graduação do ICMC- USP.

São Carlos, julho de 2000.

Regilene Delazari dos Santos Oliveira 